\title{
USING DIGITAL DEVICES IN CLASSROOM LEARNING: A COMPLEXITY THEORY PERSPECTIVE
}

BY

\section{RANA DAOUD}

\author{
A thesis \\ submitted to the Victoria University of Wellington \\ in fulfilment of the requirements for the degree of \\ Doctor of Philosophy
}

Victoria University of Wellington

2020 


\begin{abstract}
Using digital devices in primary classrooms has become a common practice in many schools around the world. A considerable amount of research has explored this phenomenon. However, the majority of studies published in highly ranked educational technology journals have focused on the effectiveness of interventions related to using digital devices in primary classrooms and few studies examine students' use of digital devices during learning which reflects a dominance of the reductionist approach in studying classroom environments. While there is rich literature addressing the outcomes of using devices, much is still unknown about the use itself.
\end{abstract}

The present study aimed to understand how students use digital devices in classroom learning activities. It explored the ways of use, the factors that shaped these uses, and how the uses contributed to the purposes of learning activities. Approaches used to study phenomena in open recursive systems, such as classrooms, should be different from approaches used to study phenomena in closed systems under controlled conditions. Therefore, Complexity Theory provided a framework to understand the socio-materiality of digital devices in classrooms learning. In addition, Actor-Network Theory was used to study the phenomenon in action and Biesta's (2009) framework of the functions of education to understand the contribution of the uses to educational purposes.

This multiple case study was conducted in New Zealand within two schools where two teachers and seven upper-primary students participated in the study. Data were collected across six months through observations, semi-structured interviews, group interviews, informal conversations, student think-aloud interviews and artefacts from learning including video screen-captures. Data were coded and analysed using the thematic analysis and an abductive strategy.

From a synthesis of the findings a 'Using Devices for Classroom Learning' model was developed in which seven patterns of use were identified. The children used their devices as a source of information, means of communication, production medium, external personal memory, collective memory coordinator, trial-and-error learning space, and as a research tool. Interconnected factors shaped these uses which were related to the educational system, school and classroom environment, teachers, students, and digital technologies. The findings showed how the seven uses contributed to the educational purposes of classroom learning 
which were classified into students' qualifications, socialisation, and subjectification.

However, some of these uses led to undesirable influence on students' learning.

This study provides theoretical and practical contributions to the field of using digital technologies in education. Complexity thinking, as a holistic approach, sheds light on blind spots of the educational process and acknowledges the complexity and uncertainty when using devices for learning in social complex systems such as classrooms. What emerges in classrooms does not result from separate factors but from a network of relationships and interactions of interconnected factors. The model developed provides an analysis tool for researchers and assists educators and policymakers to understand and anticipate the role of digital devices in classroom learning. 


\section{Acknowledgements}

First and foremost, thank you, God, for being with me during this journey, which has been, by far, the hardest and the most special experience I have ever been through. Thank you for supporting me and surrounding me with people, or gifts, to whom I will always be thankful and grateful.

I am deeply indebted to my supervisors, Dr Louise Starkey and Dr Hiria McRae, whose generous advice, precise feedback, and deep questions helped me to complete this thesis. No matter how stressed or anxious I was, your humanity and encouragement put a smile on my face, gave me confidence, and inspired me to continue and become a better educator in future.

I profoundly appreciate the financial support, scholarship, and services I have received from Victoria University of Wellington through the Wellington Faculty of Graduate Research, Wellington University International, and Student Learning. I would like to extend thanks to the Wellington Faculty of Education and School of Education staff and colleagues for being a warm and caring learning community. Thank you also to the Wellington UniVentures team, especially Jeff Howe, Doyet Sevilla, Elizabeth Craker, and Kieu Pham who provided a supportive and encouraging work environment. Special thanks to my friends Marian, Anna-Marie, Ethel, Jorge, Liwei, Anne, Autumn, Meera, Ganlong, Bruce, Suzanne, Kaitlin, Brent, Bambi, Renu and all the weekend thesis writing-Bootcamps and writing sessions friends from all disciplines and countries; with you, I spent the most beautiful and productive writing times during my studies. Thanks to my friends Tho Vo and Mehri Irajzad who always encouraged and supported me. To Madeleine Collinge, I am sincerely thankful for proofreading my thesis.

This thesis is dedicated to those for whom words are never enough to thank. First, to the schools, teachers, and students who welcomed me into their classrooms and shared their gold, wisdom, and knowledge that made this research possible. Second, to my beloved family: my dear husband Suleiman and daughters Noor and Somaia in Palestine who were extremely patient, supportive, and resilient while I was away from them. It is dedicated to my sons Omar, Ali, and Othman, whose love, understanding, sweet words, and evening cups of tea gave me the power to persist. I can’t forget my brother Rami and his family Rana, Hadi, and Noor who supported me financially and emotionally in New Zealand. 
Last but not least, this thesis is dedicated to the memory of my father Mohammed, my mother-in-law Fatimah, and my mother Ibtisam whose last words before she died in my first year of candidature were "I'm sure you can do it". 


\section{Table of Contents}

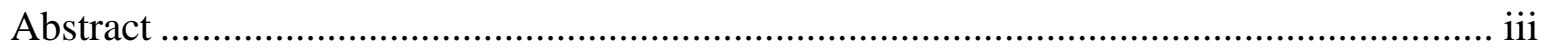

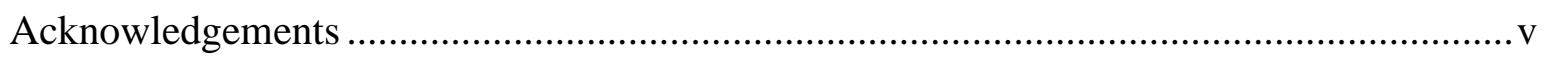

Table of Contents ...................................................................................................................

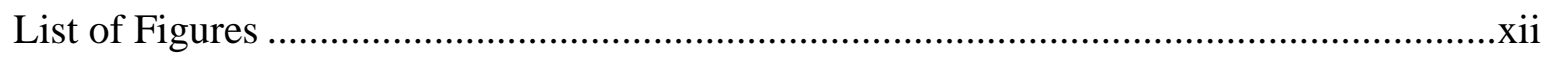

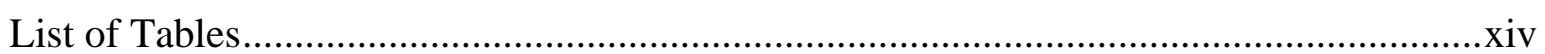

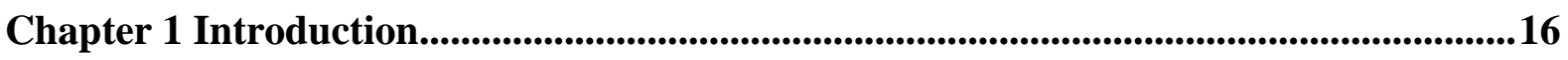

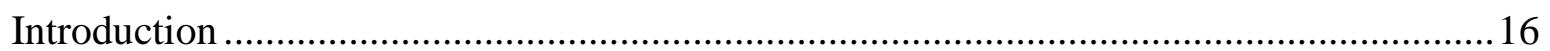

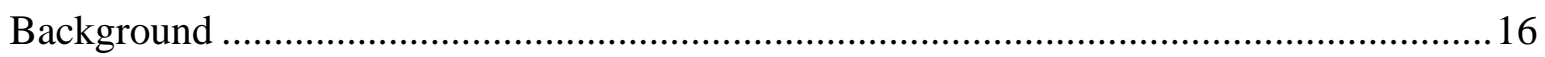

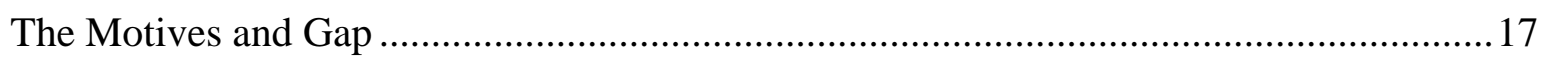

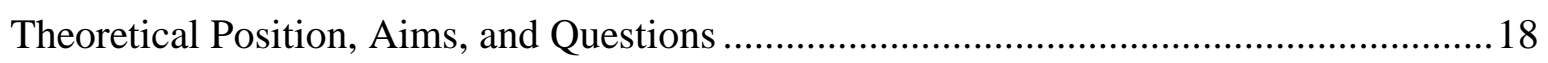

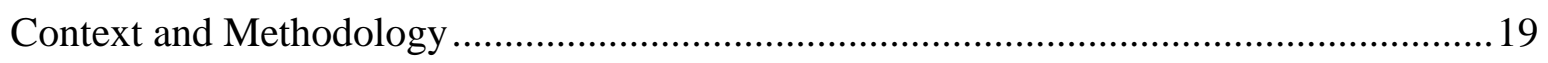

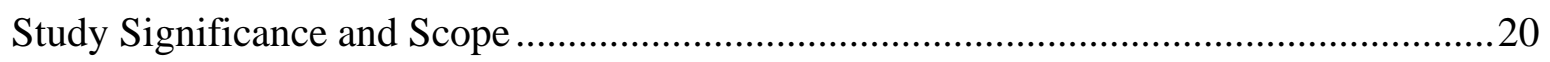

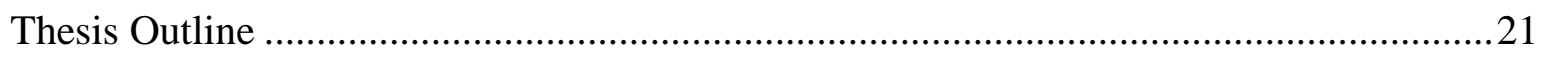

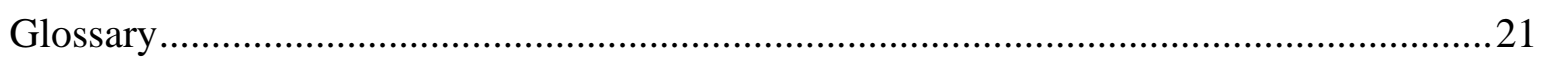

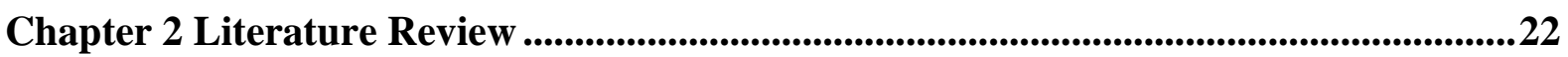

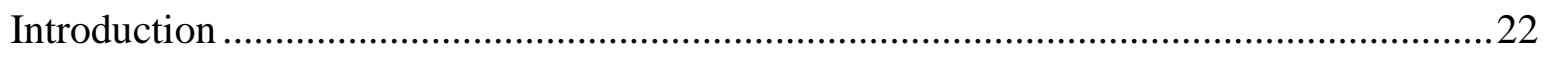

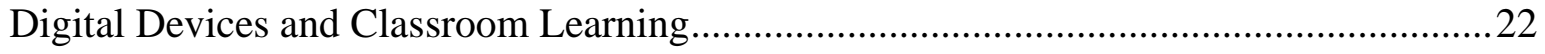

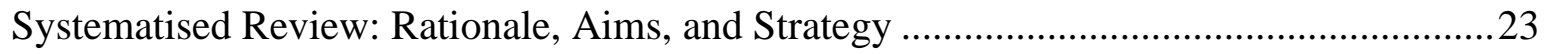

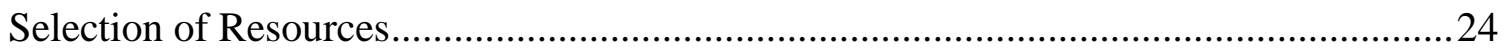

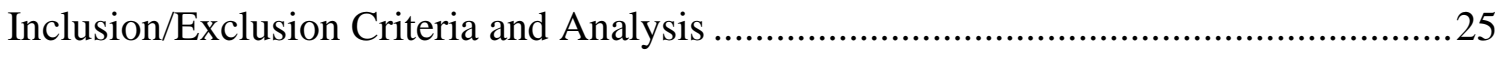

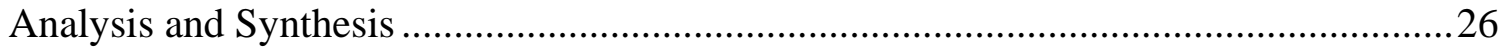

Approaches and Focuses of Research on Digital Devices .............................................. 30

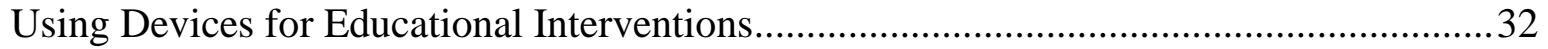

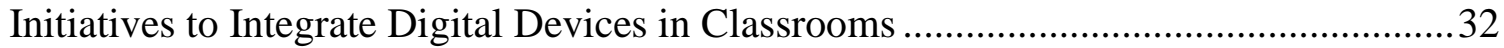

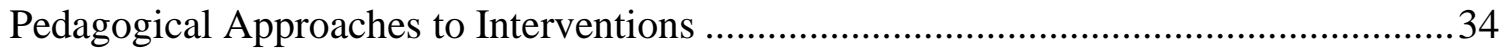

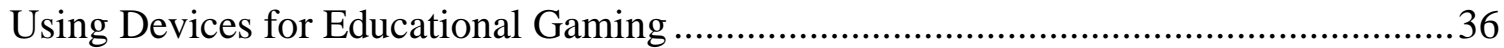

Using Devices in Comparison to Traditional Methods ................................................... 37

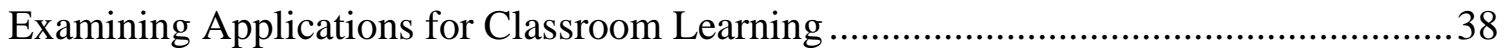

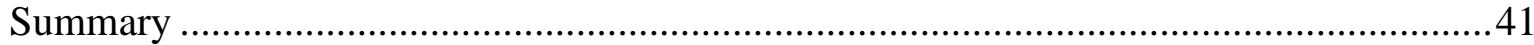

Students’ Experience When Using Digital Devices.......................................................... 41

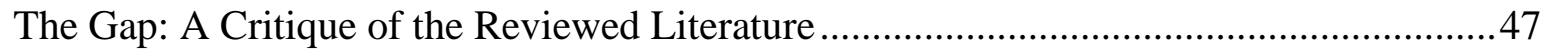

Studying the Socio-materiality of Digital Devices in Classroom Learning ........................49

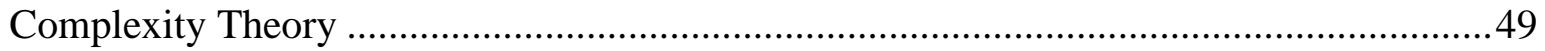

1. A Complex System is 'Systemic and Synergistic' ......................................................50 


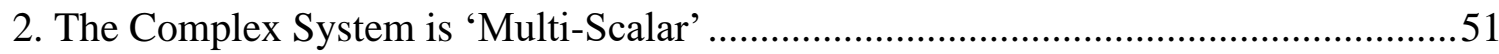

3. Resilience and Adaptability Results from Diversity, Variation, and Fluctuations........52

4. Context, Events, and History Shape the Future .............................................................52

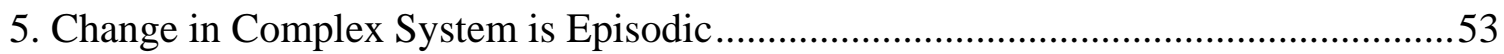

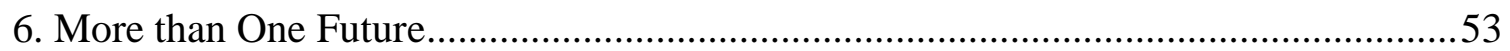

7. Self-Organisation, Self-regulation, and Emergence in Complex System .....................54

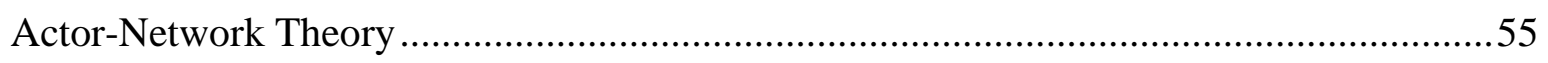

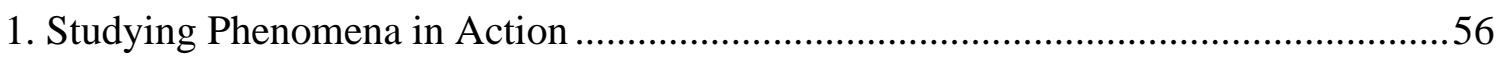

2. Actor, Network, and Results are Inseparable ........................................................57

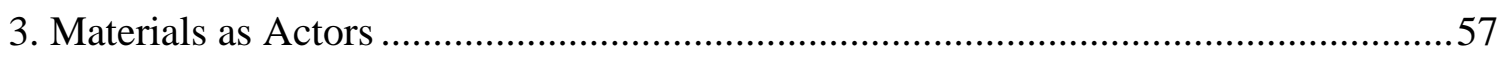

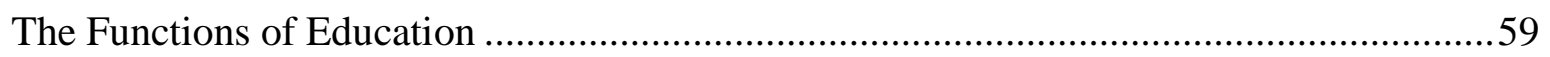

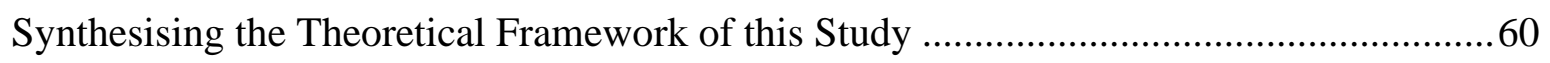

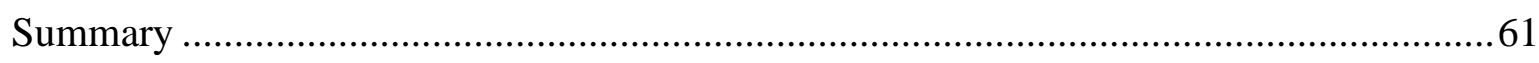

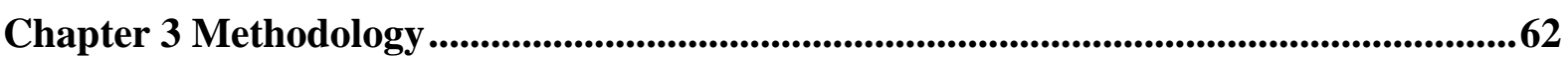

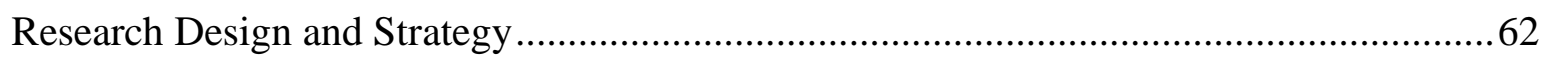

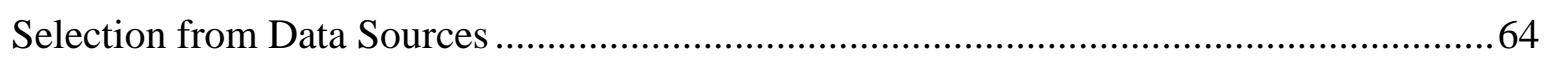

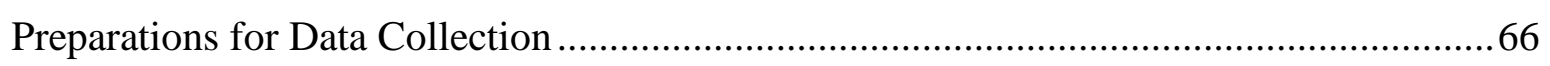

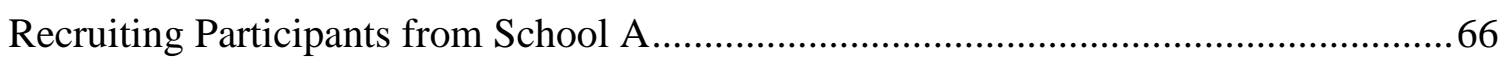

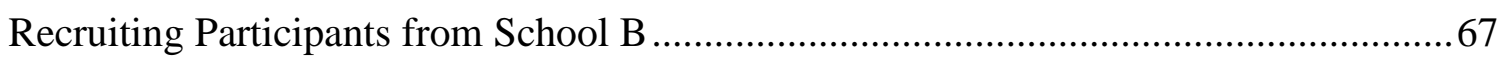

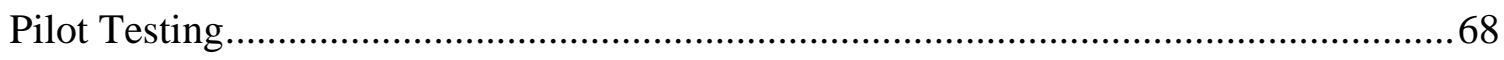

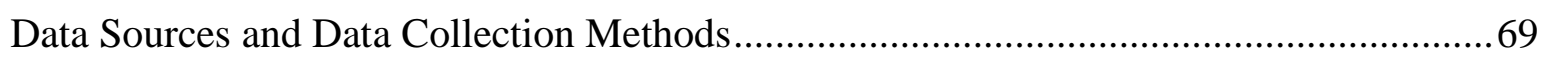

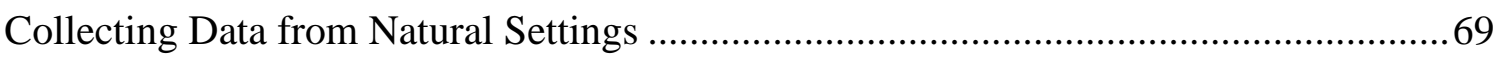

Collecting Data from Semi-Natural Settings ........................................................... 71

Collecting Social Artefacts and Documents .............................................................. 73

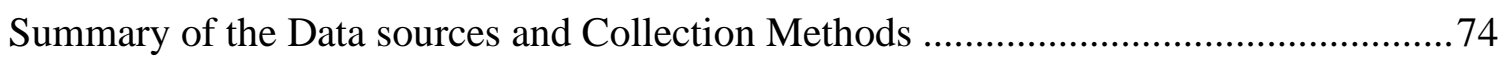

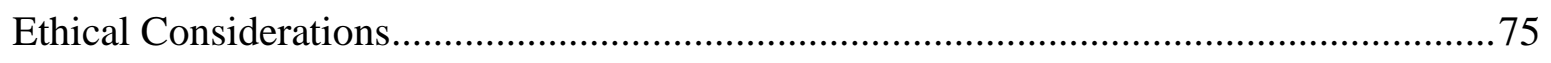

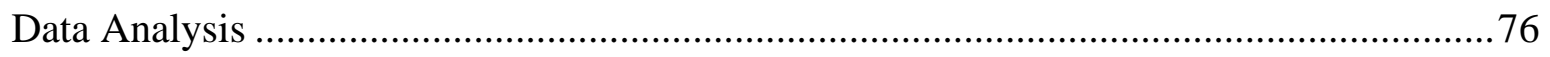

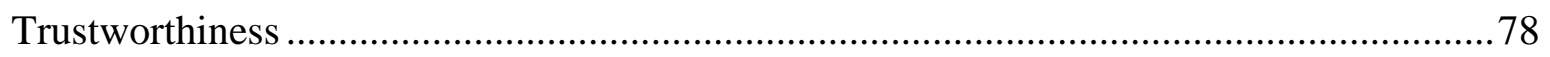

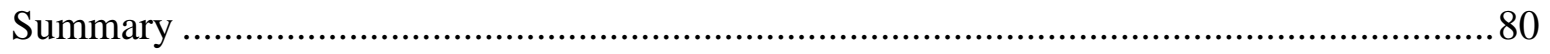

Chapter 4 Case Study One .............................................................................................

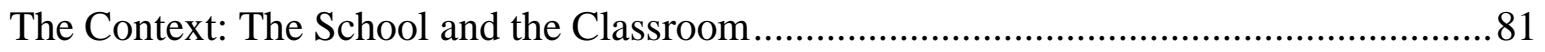

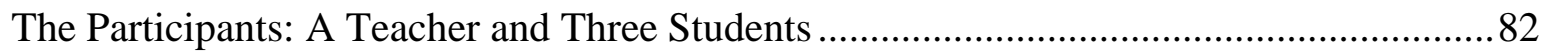

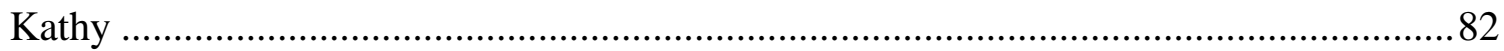

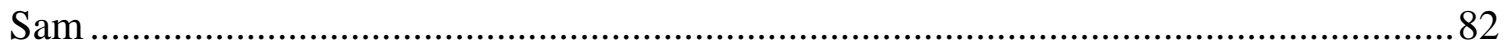

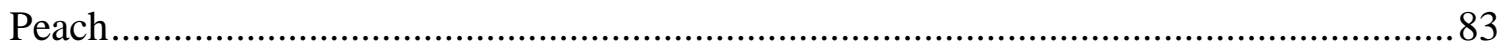


Mark. .83

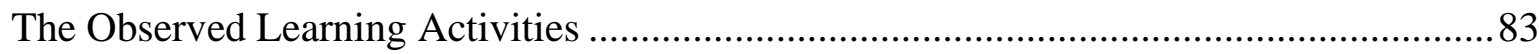

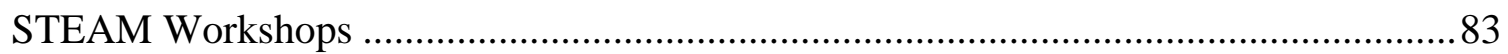

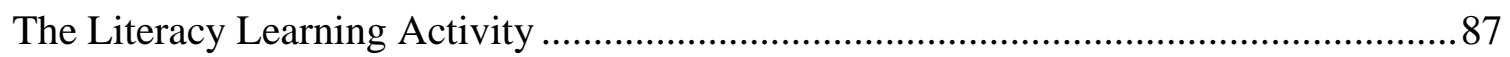

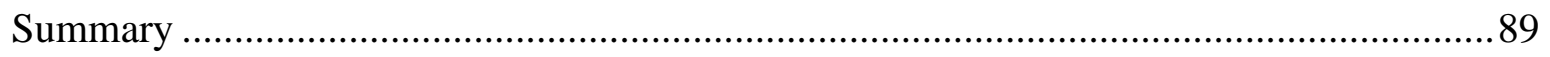

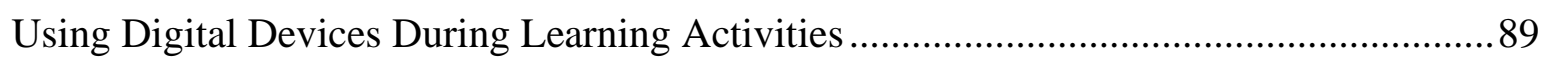

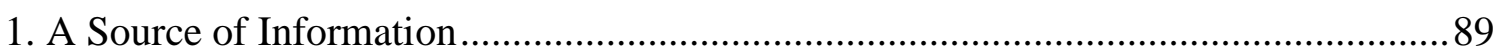

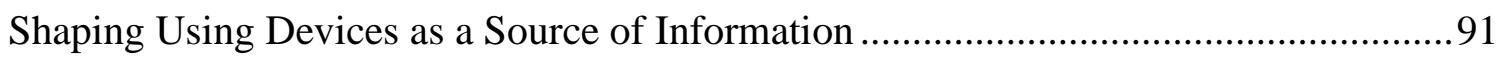

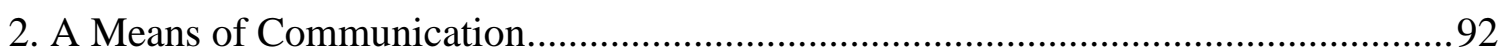

Shaping Communications About and Through Devices ............................................... 94

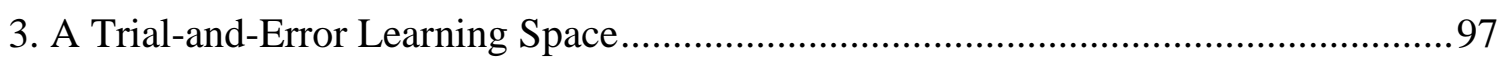

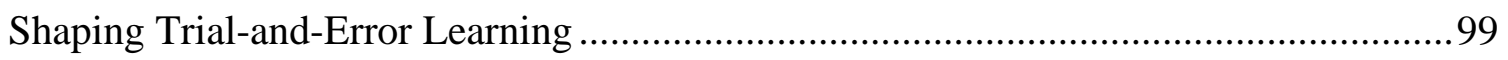

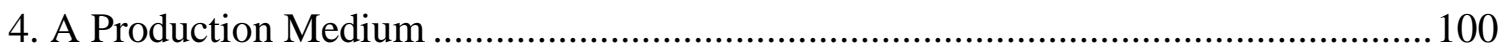

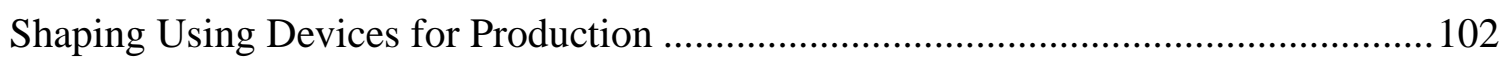

5. An External Personal Memory ............................................................................ 107

Shaping Using the Devices as Personal Memory ........................................................ 108

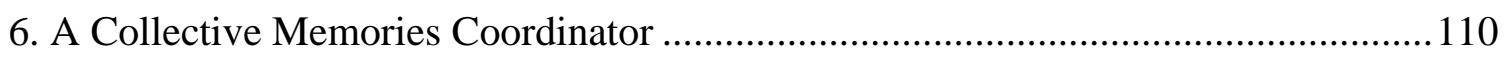

Shaping Creating Collective Memories.................................................................. 112

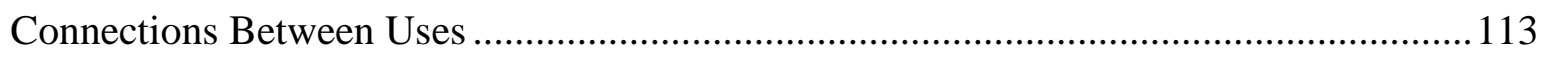

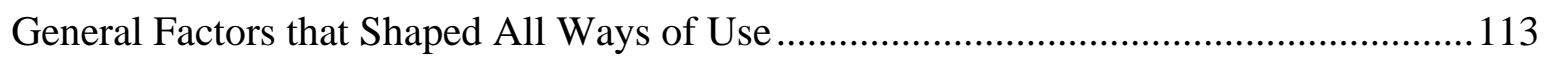

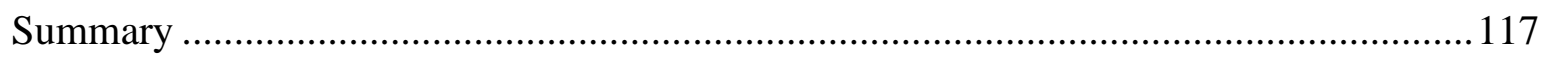

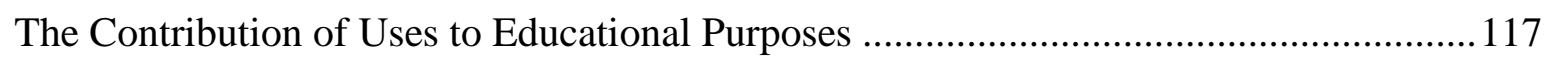

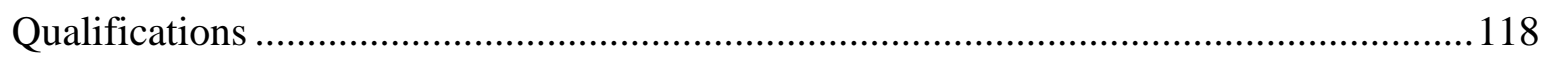

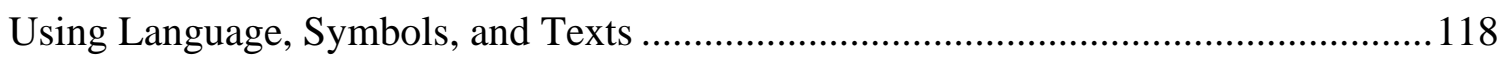

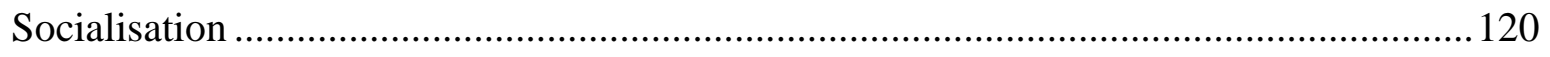

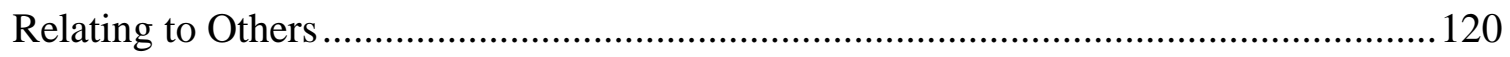

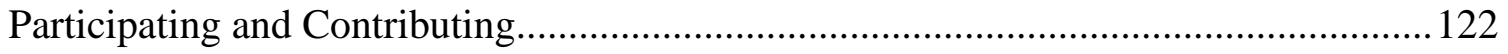

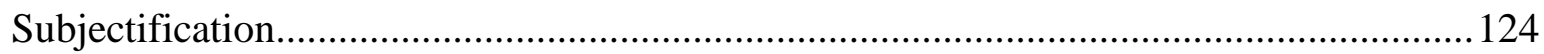

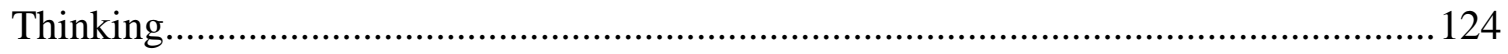

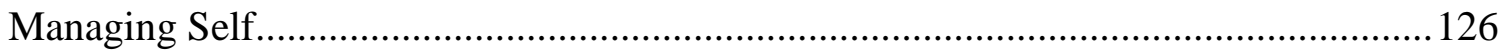

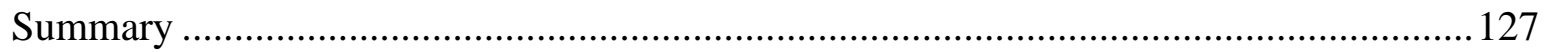

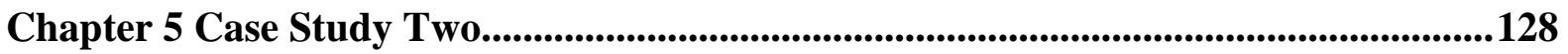

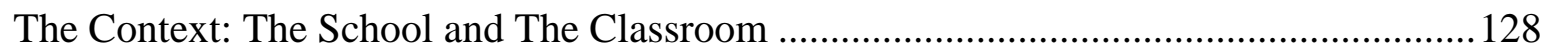

The Participants: A Teacher and Four Students .................................................................... 128

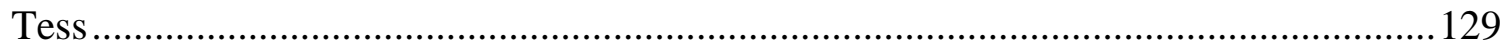


Bob. 129

Mitchel 130

Alice. 130

Ian 131

The Observed Learning Activities 131

Scientific Research Learning Activity 131

The Leadership Activity 134

Summary 136

Using Digital Devices During Learning Activities ..................................................... 136

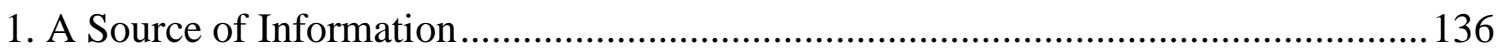

Shaping Using Devices as a Source of Information .................................................. 139

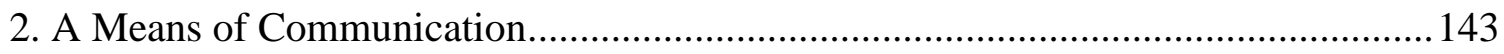

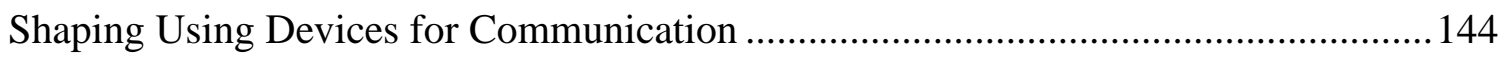

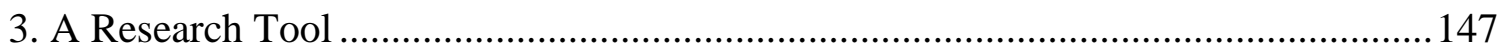

Shaping Using Devices as a Research Tool ............................................................ 149

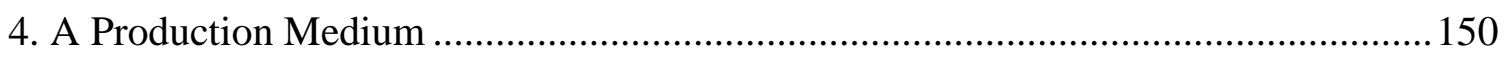

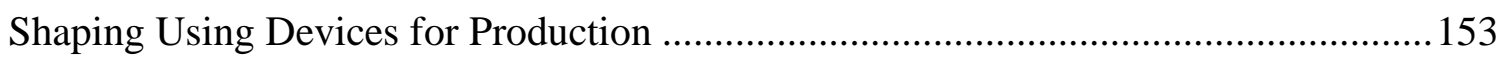

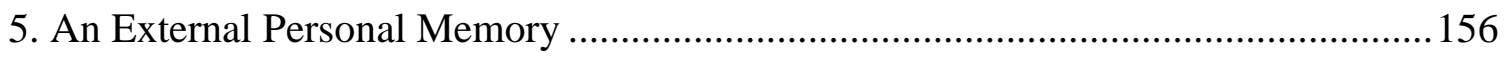

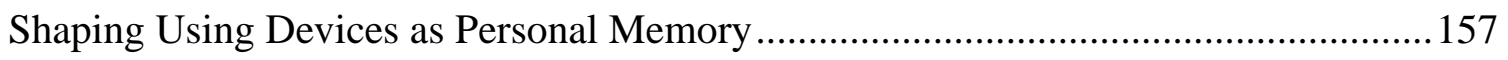

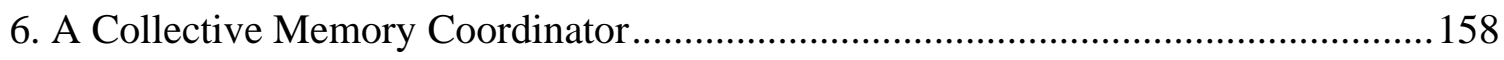

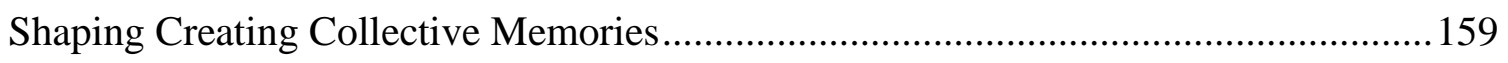

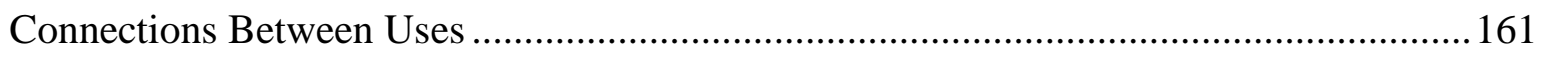

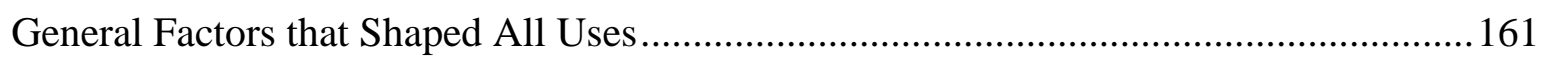

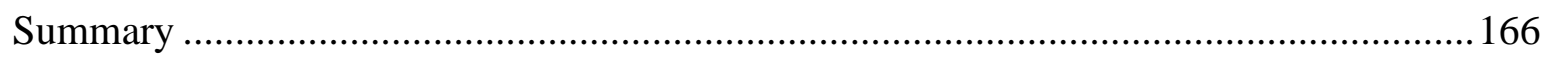

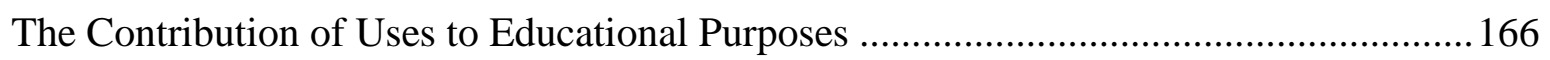

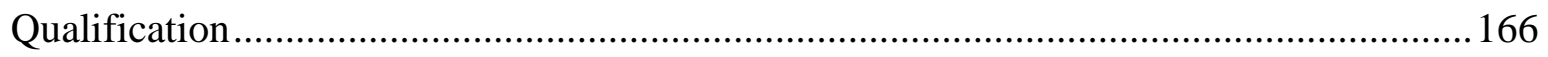

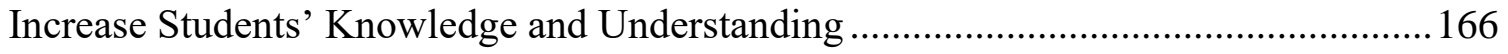

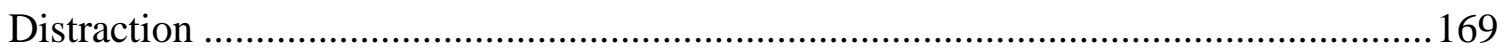

Understanding and Undertaking Multiphase Projects ................................................ 170

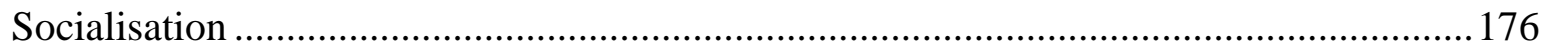

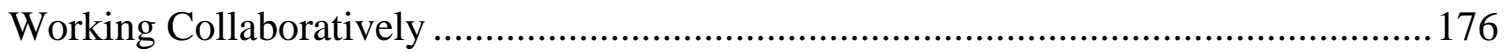

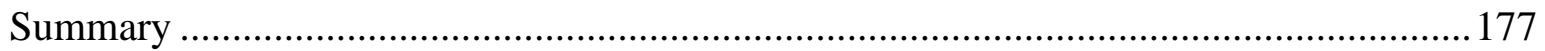

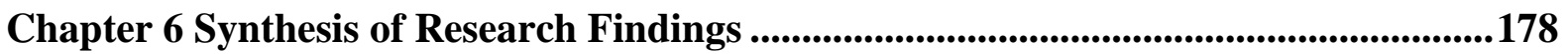

1. In What Ways Do Primary Students Use Their Digital Devices in Classroom Learning Activities?.. 
2. What Factors Shape the Ways of Use in Classrooms?.

181

3. How Do the Ways of Use Contribute to the Educational Purposes of Classroom Learning Activities?.

How Do Students Use Digital Devices for Educational Purposes in Primary Classrooms? 188

Chapter 7 Discussion and Conclusion. .190

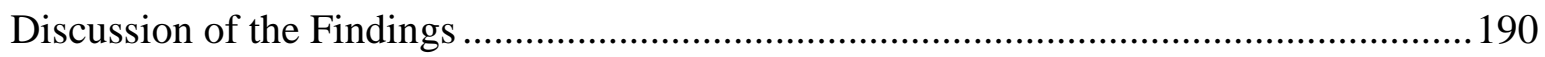

Using Devices in Classrooms: Emergence and Self-Organisation ................................... 190

Ways of Use Explicitly Reported in Educational Research ........................................ 191

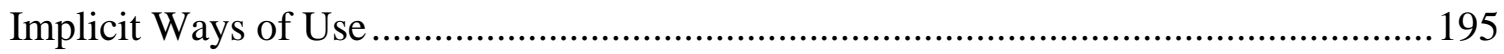

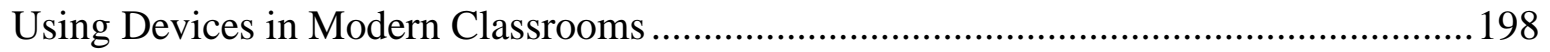

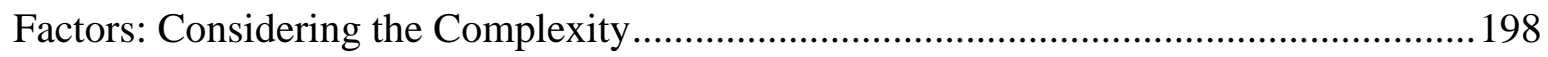

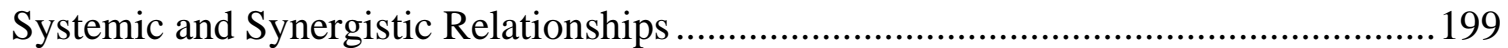

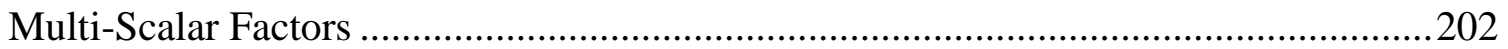

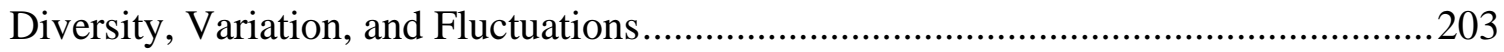

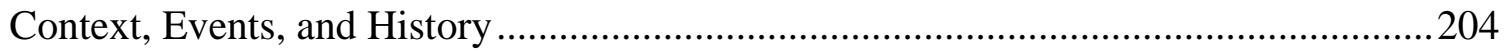

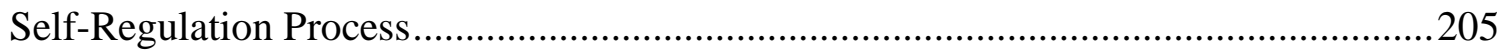

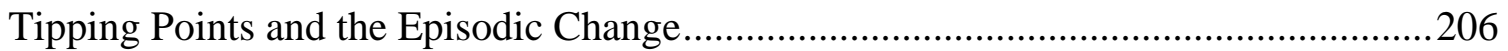

Contributions to Educational Purposes: More Than One Future ......................................207

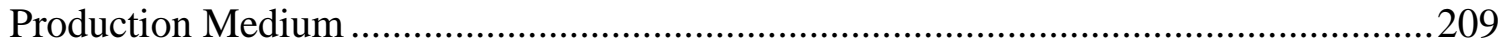

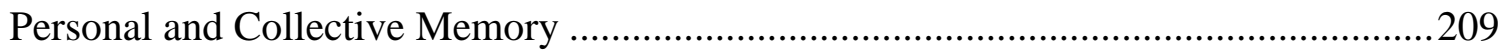

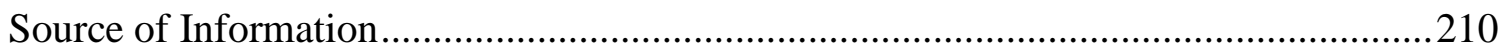

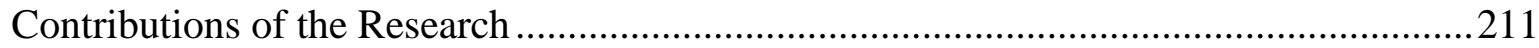

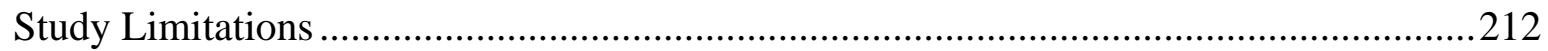

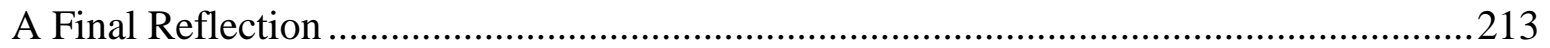

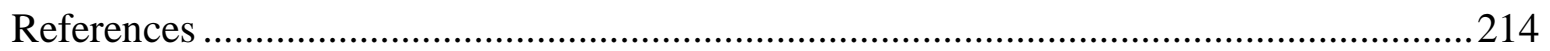

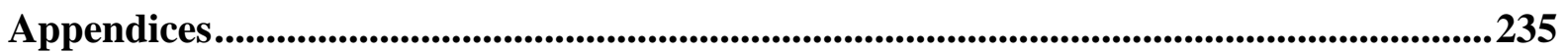

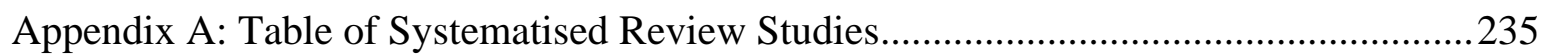

Appendix B : Information Sheet and Consent Forms for Teachers .................................2. 239

Appendix C: Information Sheet and Consent Forms for Students..................................243

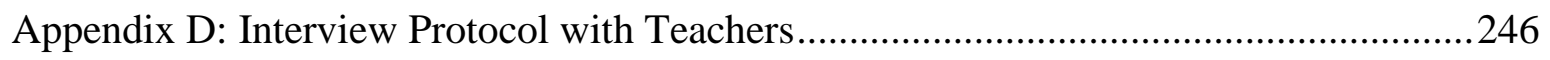

Appendix E: Group Interview Protocol with Students and Think-Aloud Prompts............249 


\section{List of Figures}

Figure 2.1 Illustration of the Study's Symbol .27

Figure 2.2 Classification of the Studies Based on Focus.................................................29

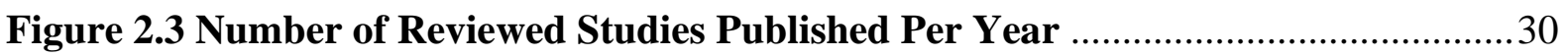

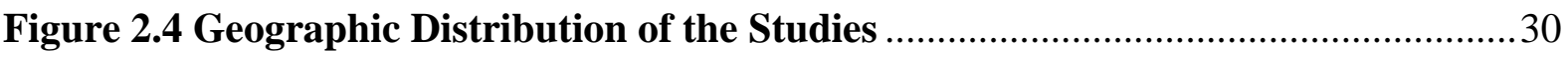

Figure 2.5 Number of Studies Investigated Using Devices for Subject Areas..................31

Figure 2.6 Number of Studies Reported each Type of Included Devices .........................31

Figure 2.7 Numbers of Studies in Terms of Their Focus, Design, and Methods ............32

Figure 2.8 The Functions of Educations Based on Biesta's Framework .........................59

Figure 4.1 Sample of the Weekly Table of STEAM Workshops ....................................... 84

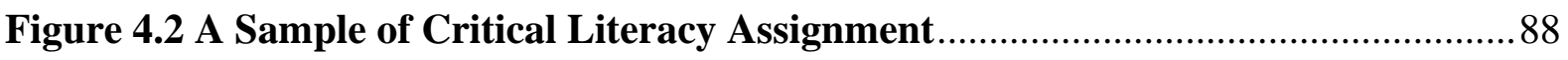

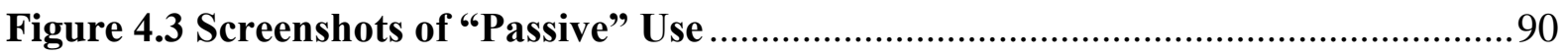

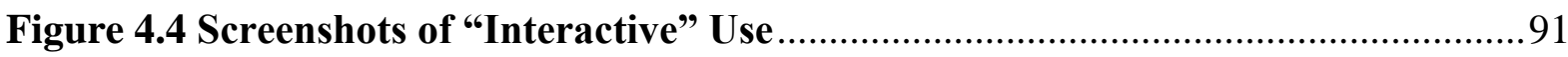

Figure 4.5 Communication Through Devices for Feedback .........................................93

Figure 4.6 Screenshots of Features Used During the Trial-and-error Learning ............99

Figure 4.7 Samples of Students' Critical Literacy Assignments ................................. 102

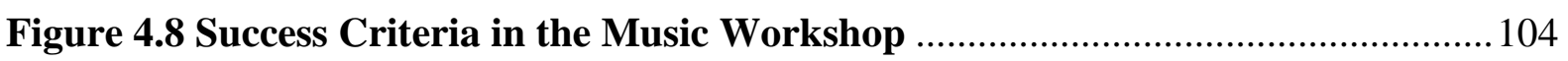

Figure 4.9 Teachers' Samples and Students' Products in STEAM Workshops ............ 105

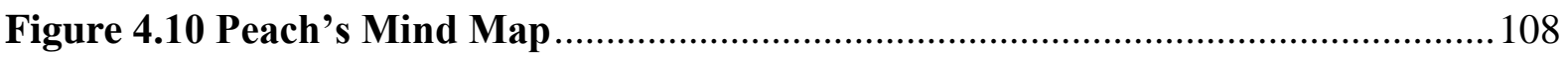

Figure 4.11 Detailed Instructions about Saving and Recording Students' Work.......... 109

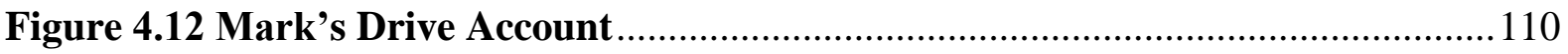

Figure 4.13 Mark when was Typing Simultaneously with his Friend ...........................111

Figure 4.14 Participants Using Language, Symbols and Text .................................... 119

Figure 4.15 Sam Receiving and Asking for Feedback ........................................... 121

Figure 4.16 A Teacher's and a Student's Feedback on Mark's Blog............................ 122

Figure 4.17 Peach Writing with her Group on the Same Document …........................ 123

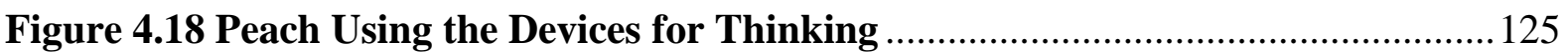

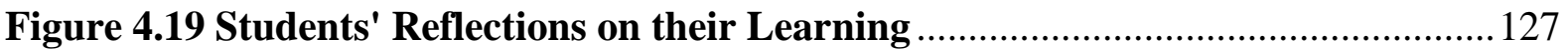

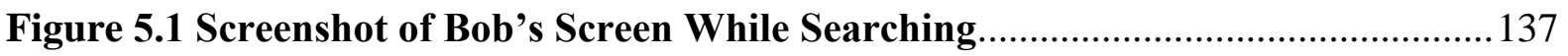

Figure 5.2 Using Devices as a Source of Information by Alice .................................... 138

Figure 5.3 Using Devices as a Source of Information by Ian ........................................ 138

Figure 5.4 Teacher's Instructions to Use the Devices as a Source of Information......... 139

Figure 5.5 Instructions Relate to Using the Device as a Source of Information ............140

Figure 5.6 Suggested Spelling in the Search Engine ................................................... 141 
Figure 5.7 Ian Using the Pop-up Feature. 141

Figure 5.8 Screenshot of Bob while sending a Google Form to a Teacher in her School

Figure 5.9 Instructions on Using Devices for Communication .................................. 146

Figure 5.10 The Procedure of Bob's Scientific Research ............................................ 147

Figure 5.11 The Procedure of Mitchel's Scientific Research ........................................ 148

Figure 5.12 Using Devices to Analyse Data by Bob .................................................... 149

Figure 5.13 Instructions on Using Devices as a Research Tool....................................150

Figure 5.14 Bob's Display Board for the Science Learning Activity............................151

Figure 5.15 Ian's Display Board for the Science Learning Activity ..............................151

Figure 5.16 Bob's Screen Capture while Creating a Google Form .............................. 152

Figure 5.17 Instructions on Producing Scientific Research Presentation ...................... 154

Figure 5.18 Bob Using her Search History to Find Information .................................. 157

Figure 5.19 Instructions Related Managing Personal and Collective Memories........... 158

Figure 5.20 Bob while Creating a Collective Memory................................................. 159

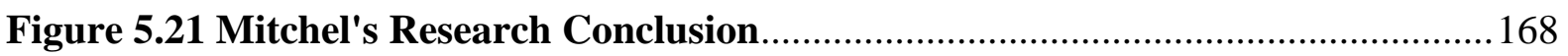

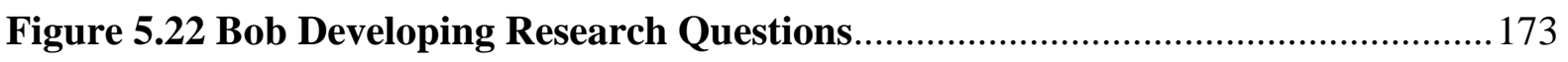

Figure 5.23 Using Devices as a Source of Information to Create Models...................... 175

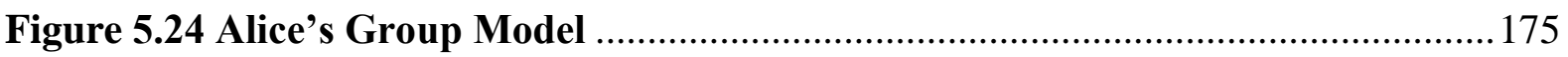

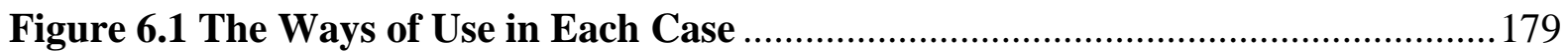

Figure 6.2 Interconnected Factors Shaping Using Devices in Classroom Settings ........ 181

Figure 6.3 The Contribution of Uses to Educational Purposes in the Two Cases ......... 186

Figure 6.4 Using Devices for Classroom Learning Model (UDCL) ............................... 189

Figure 7.1 The Interconnected Elements and the Focus of the Current Study............. 199 
List of Tables

Table 2.1 Selected Journals for the Systematised Review ............................................25

Table 2.2 Retrieved and Selected Numbers of Studies ................................................26

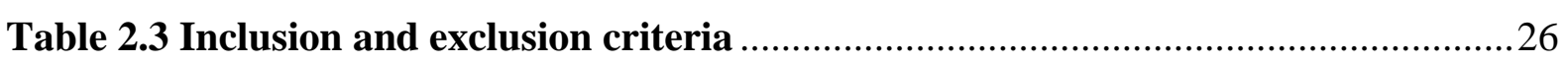

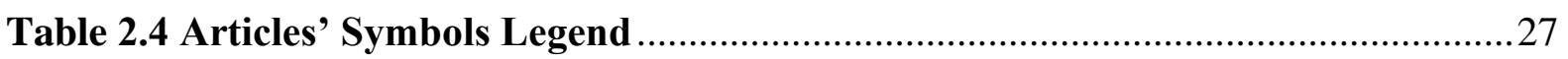

Table 2.5 Summary of Research on Students' Use of Digital Devices ............................46

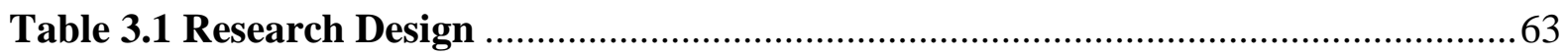

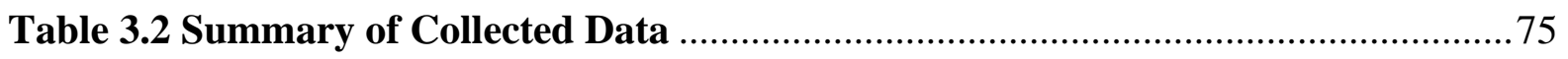

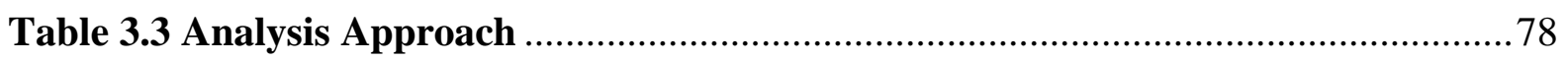

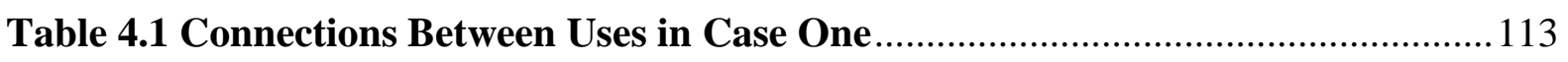

Table 5.1 Connections Between Uses in Case Two ...................................................... 161

Table 6.1 A Summary and synthesis of Connections Between Uses of the Two Cases .179

Table 6.2 Identification of the Ways of Use of Digital Devices for Classroom Learning

Table 6.3 General Factors that Shaped Using Digital Devices in Classrooms ............... 182

Table 6.4 Factors Shaped Using Devices as a Source of Information ............................ 183

Table 6.5 Factors Shaped Using Devices as a Mean of Communication ........................ 183

Table 6.6 Factors Shaped Using Devices as a Production Medium .............................. 184

Table 6.7 Factors Shaped Using Devices as a Personal Memory ................................... 184

Table 6.8 Factors Shaped Using Devices as a Collective Memory Coordinator ............ 185

Table 6.9 Factors Shaped Using Devices as a Trial-and-Error Learning Space ........... 185

Table 6.10 Factors Shaped Using Devices as a Research Tool ................................... 185

Table 6.11 Synthesising the Contribution of the Ways of use to Educational Purposes 


\section{Chapter 1 Introduction}

\section{Introduction}

This study seeks to understand how primary students use their digital devices in classrooms and how the ways of use contribute to the purposes of the learning activities. This qualitative study was underpinned by Complexity Theory to understand the socio-materiality of the devices. The findings led to developing a model that defines the ways of use, identifies the factors shaping the uses, and identifies how the uses could contribute to the educational purposes of classroom learning. In this introductory chapter, I first provide a background of the topic, followed by clarifying the motives of the study and the gap in the literature. Then I state the research aims and questions, the approach and context of the study, and the significance of the study. In the last section of this chapter, I briefly outline the following chapters of the thesis.

\section{Background}

The influence of digital technologies on modern societies is broad and deep. Digital technologies can be defined as the "electronic tools, systems, devices and resources that generate, store or process data" (Loong \& Herbert, 2018, p. 1). These technologies include hardware components such as mobile devices, software components such as programs and applications, and practices such as programming. Each of these components can be considered as 'singular' technology on its own even though they depend on each other to function (Arthur, 2009); for example, we need programming expertise to create applications that function on specific devices and vice versa.

While digital technologies are being continuously and rapidly developed by humans, these technologies are reshaping different aspects of life including learning and teaching in contemporary societies (Starkey, 2012). The way digital technologies facilitate access to information, instant interactions, and communication has changed society's needs and consequently changed educational objectives, policies, and practices. This can be seen as digital competency and digital literacy have become part of the educational objectives in different educational systems such as New Zealand and Australia (Starkey \& Finger, 2018). That has led to profound changes in educational policies all over the world.

Many projects and initiatives have been launched to integrate digital technologies in formal education (Ale et al., 2017; Heinrich et al., 2020; Kennedy et al., 2016). International 
comparison studies showed that new government policies had been set up to integrate digital technologies in the educational systems in both developed and developing countries (Tan et al., 2017). The integration took different forms; for example, in secondary education, it is prevalent to teach computing skills, such as programming basics and algorithms, as a discrete subject. In primary education, the common approach is to teach digital skills combined with other subjects such as teaching how to use the Internet and presentation applications for science projects (Sturman \& Sizmur, 2011). By integrating digital technologies, teaching and learning approaches that are associated with or depending on using digital technologies are evolving such as blended learning and mobile learning (Crompton et al., 2019).

Recently, using digital technologies has become a common practice in many primary classrooms (Sung et al., 2016). Many schools provide digital devices for students or allow them to bring their own technologies to schools after Bring Your Own Device (BYOD) policies were encouraged in many countries such as New Zealand (Starkey \& Finger, 2018), Europe (Ottestad \& Gudmundsdottir, 2018), and the United States (Scholz, 2016). There is a wide range of digital devices that are used in diverse ways in classrooms, such as interactive whiteboards, laptops, tablets, and smartphones (Armstrong, 2014). Concurrently, there are various applications that can be used for educational purposes as well as numerous specialised software types developed for specific educational purposes. In addition, Internet access enables students to interact with others, inside and outside their schools, and reach different resources while they are in their classrooms. The presence of digital technologies in classrooms has evoked researchers and educators to question, explore, and understand the implications for learning and teaching.

\section{The Motives and Gap}

It is my personal experience of working as a computer teacher for primary students that has driven this research. While working as a teacher, I was able to recognise different elements affecting the learning process including, but not limited to, me as a teacher, curriculum, instruction, students, and devices. There was always a high chance of surprising or unexpected outcomes, which increased my curiosity to understand how the outcomes happen and why. Understanding how young children use their devices and how that contributes to their learning was a crucial topic to explore for my teaching practices. I became more interested in research about learning and teaching in the digital age as there appeared to be a gap in what is known. 
Although extensive research has been carried out related to using digital devices in classrooms, I was not able to find sufficient answers about how children use their devices for classroom learning. The gap, discussed at length in the literature review chapter, can be summarised by arguing that many studies have focused on the effectiveness of using digital devices in classrooms (Chauhan, 2017; Tingir et al., 2017). However, there is still much unknown about what exactly happens when the learners use them for learning (Lai, 2018). Even in the contexts where integrating digital technology began earlier than other countries, such as Europe, research does not tell us much about "ways of use of technology in schools" (Ottestad \& Gudmundsdottir, 2018, p. 1347).

In general, there is a "blindness toward the question of how educational practice is affected by materials" (Sørensen, 2009, p. 2) and digital devices are not an exception. A reason for the gap can be attributed to the approach used to explore this phenomenon. The predominant approach in scientific research adopts the reductionist paradigm, which means, for example, when studying the learning process each component tends to be analysed separately (Ennis, 1992). This led to a focus on some 'snapshots' of the learning process such as learning outcomes without investigating what elements and dynamics influenced their emergence (Hurford, 2010). This explains the tendency of the studies to measure or examine the effectiveness and outcomes of using devices, not the use itself. Therefore, too little attention has been paid to conceptualising the uses, identifying the factors that shaped using digital devices in classrooms, and how that might contribute to the desired outcomes.

\section{Theoretical Position, Aims, and Questions}

To address the gap in the literature, this study adopts Complexity Theory by considering classrooms as complex adaptive systems while focusing on the dynamics related to using digital devices by students. Different scholars considered classrooms as complex systems where many elements interact in different ways (Davis \& Sumara, 2006; Hurford, 2010). Complexity Theory can highlight critical moments and interactions, which are usually not examined, that can reshape and change the learning process as a whole (Ennis, 1992). Digital devices are one of these elements classified as materials interacting with the social actors. Fenwick (2010) suggests that Complexity Theory is one of the approaches suitable to study the socio-materiality of the 'things' in the learning environments. She outlined that sociomaterial approaches are the approaches that investigate the critical role of the material in reshaping the components, relationships, and interactions in different phenomena in the social world such as learning. She presented three reasons to justify the importance of focusing on 
the socio-materiality that can be applied to digital devices. First, digital devices are becoming an integral part of many classrooms today. Second, these devices are interacting with other elements, comprising learning processes in different ways in different contexts. Third, studying the socio-materiality of digital devices helps to eliminate the artificial distinctions in social research (e.g. formal/informal, individual/collective) that obstruct the understanding of entangled phenomena. Therefore, using Complexity Theory can give a holistic view of dynamics and the factors interacting in the classrooms that affect the success or failure of integrating digital devices.

The other approach adopted in this study was looking at the contribution of the devices to the educational purposes instead of measuring the impact of using devices (Biesta, 2015). The rationale behind this approach was that what emerges in the complex systems, such as classrooms, results from entangled factors that are constantly shaping each other (Davis \& Sumara, 2006).

The main purpose of this study was to develop an understanding of the use of digital devices by primary students, what factors shape the uses, and how that contributes to the educational purposes of classroom learning activities. In particular, this thesis seeks to address the following question: How do students use digital devices for educational purposes in primary classrooms? To answer this question, three sub-questions were addressed:

1. In what ways do primary students use their digital devices in classroom learning activities?

2. What factors shape the ways of use in classrooms?

3. How do the ways of use contribute to the educational purposes of classroom learning activities?

\section{Context and Methodology}

This study took place in New Zealand, which is one of the leading countries in terms of the quality of primary education (Schwab, 2016)). In an endeavour to obtain better performance, teaching, and learning, schools in New Zealand were given a high level of autonomy compared to other educational systems in the world (Hood, 2019). Schools are self-managed and administrated by elected Boards of Trustees, most of whom are parents. The New Zealand Curriculum (Ministry of Education, 2007) provides a framework that outlines the direction and objectives of the educational process, but each school has to develop its own curriculum. The school curriculum should include a detailed plan, resources, and models that 
align with the national curriculum. Teachers in every school have the agency as well as the responsibility to develop and implement instructional strategies, learning activities, and materials to achieve the objectives of the school and national curriculums. Schools are also required to develop their plans to integrate digital technologies guided by the national curriculum and based on their contextual needs (Starkey \& Finger, 2018). Using digital devices is becoming a common practice in primary classrooms (Lips et al., 2017). Still, some schools strive to provide a device for each student either by providing options to lease or purchase through trusts established to serve low socio-economic contexts (Starkey \& Finger, 2018). For the purposes of this study, the diversity of schools' interpretation of the national curriculum and the diversity of learning activities integrating digital technologies provide a unique opportunity and a variety of choices to study different learning experiences.

This thesis was conducted in two classrooms in two schools; each was studied as a complex system. The case study design was adopted to develop a thick description of the dynamics that happened during learning activities (Blaikie, 2009). Data is gathered via semistructured interviews, group interviews, observations, video screen captures, think-aloud interviews, and analysis of participants' artefacts. In-depth analysis was conducted after data transcription and coding. The findings generated a conceptualisation of seven ways of using devices, classification of the factors shaping the uses, and linking the uses with possible educational purposes. The syntheses of the findings resulted in developing a model that provides an understanding of the dynamics of the uses in classroom learning.

\section{Study Significance and Scope}

This study provides an important opportunity to advance the understanding of device use through the lenses of Complexity Theory, which is an evolving, yet promising, holistic theoretical perspective in educational research. This study aims to contribute to the growing area of research that explores the socio-materiality of digital devices in classroom learning activities (Fenwick et al., 2015). There are several important areas where this study makes an original contribution including conceptualising the uses and developing a model that illustrates the dynamics of the uses. Therefore, this study offers researchers alternative theoretical and methodological insights into studying this phenomenon.

On the practical side, this study provided a detailed description of how students use digital devices during learning activities. That can help teachers and educators to reconsider 
the factors that may affect their own context and enhance making decisions, planning, and utilising these devices in their contexts.

The reader should bear in mind that the focus of this study is the use of devices for learning activities in formal education settings, particularly classrooms, where teachers lead the learning process. It is beyond the scope of this study to explore the use in an informal setting or in online learning settings. The study was limited to primary education, mainly upper-primary students from years seven and eight. The study was not an intervention, which means that I did not have any control or intervention related to the learning process.

\section{Thesis Outline}

This study is organised into seven chapters. The first chapter introduces the study in general. The second chapter presents a systemic review of recent studies addressing using devices in primary classrooms to identify the gap; then it lays out the theoretical dimensions and the frameworks of the study. The third chapter describes the methodology and methods used to collect and analyse data. The fourth and fifth chapters report the findings of the case studies followed by a summary and synthesis of the findings in Chapter 6 . The final chapter discusses the finding in light of the theoretical framework and literature and discusses the contributions and the limitations of the study.

\section{Glossary}

The following words are in Te Reo Māori, which the indigenous language of New Zealand and one of official languages in the country.

Hui: Gathering, meeting, assembly, seminar, conference.

Kawa: Protocol.

Kōwhaiwhai: painted scroll ornamentation - commonly used on meeting house rafters.

Pākehā: New Zealander of European descent.

Te Reo Māori: Māori Language.

Tūî: An endemic bird of New Zealand.

Whānau: Extended family, family group. 


\section{Chapter 2 Literature Review}

\section{Introduction}

This chapter reviews empirical and theoretical studies related to the scope and purpose of this study. First, I present a systematised review of studies published in the most-cited and highly ranked journals in educational technology to address the current state of knowledge and contextualise my research. Then I demonstrate the gap that this study aims to address, combined with a critique of the reviewed literature and justification of the adopted approach. After that, the chapter provides a background of the theoretical position and a synthesis of the theoretical framework that underpins the study.

\section{Digital Devices and Classroom Learning}

Digital technologies can be defined as the "electronic tools, systems, devices and resources that generate, store or process data" (Loong \& Herbert, 2018, p. 1). These technologies include hardware components such as motherboards; software components such as programs; and practices such as programming. While each of these components can be considered or studied as a 'singular' technology on its own (Arthur, 2009), the three components are interdependent in a way that they need each other to function. For example, we need programming expertise to create applications that function on specific devices and vice versa. This study looks at digital devices as a manifestation of digital technologies that comprises the three components.

Learning, which happens in different contexts with various ways and tools, has been one of the most interesting phenomena to be examined and understood. Human and nonhuman elements, such as teachers, students, pens, and books, interact together and shape the learning process (Fenwick, 2010). In the digital age, digital devices have become new elements that are widely used in formal and informal learning contexts. By using devices, the three components of digital technologies become part of the learning process; for example, students need to learn different practices and skills to deal with and utilise the software and hardware components for learning activities. Questions have been raised about how learning and teaching might be influenced by digital devices. Scholars argue that understanding the role of these technologies in the educational process is crucial to set realistic expectations and make effective decisions when using them for learning and teaching (Ellis \& Goodyear, 2013). 
A large and growing body of literature has investigated using digital devices for classroom learning. Different terminologies are used to address the phenomenon of using digital technologies in education, such as computer-assisted learning and Information Communication Technology (ICT) in education (Punie et al., 2006). Over the past decade, more types of digital devices are being used in classrooms beside PCs, such as tablets, smartphones, and laptops including notebooks and Chromebooks. A considerable amount of literature has focused on 1:1 computing programmes where each student has an individual laptop for use in classrooms (Harper \& Milman, 2016; Zheng et al., 2014). More recent literature has focused on mobile learning, which emphasises the mobility of learners when using mobile devices (Traxler \& Crompton, 2015). The wide range of terminologies used to address the phenomenon and the large volume of published studies make reviewing literature and pinpointing the gap a challenge.

Several studies have reviewed the literature on using digital devices in classrooms. Chee et al. (2017) conducted a systematic meta-analysis review to examine the trends of mobile learning research in the top six major educational technology journals based on Google Scholar metrics. Their analysis included 144 studies published between 2010-2015. Their review showed that the main focus of more than half of the studies was to evaluate the effectiveness of mobile learning. More than $50 \%$ of the studies reported positive outcomes of mobile learning while less than 5\% reported negative outcomes and about $7 \%$ reported neutral outcomes. However, most of the studies were carried out in the higher education context. Crompton et al (2017) reviewed 113 studies involving mobile learning in the K-12 settings published between 2010-2015 in 10 major educational technology journals. They have found that most of the studies focused on the effects of using mobile devices on student learning and most of them reported positive outcomes. The most common method used in the studies was questionnaires followed by pre/post-tests. Most of the learning activities (40\%) in these studies were aligned with behaviourist learning theory. For the purpose of my study, I systematically reviewed studies published in the last five years. In the next sections, I first describe the literature then I present my critique and argument related to the gap and framework adopted in the current study.

\section{Systematised Review: Rationale, Aims, and Strategy}

A considerable amount of research has explored using digital technologies in classrooms; however, different scholars have argued that our understanding of this phenomenon remains lacking (Lai, 2018; Ottestad \& Gudmundsdottir, 2018; Serdyukov, 2017). Others claim that 
the educational research tends to more value experimental methods (Biesta, 2015) and the reductionist approach (Hurford, 2010), which overlook influential factors in the learning process (Ennis, 1992; Sørensen, 2009). Therefore, I conducted a 'systematised' review of studies published in the most-cited and highly ranked journals in educational technology to identify the trends of educational research, and pinpoint the focus of the literature, the methodologies used, and the major findings. This approach enabled me to verify the claims, identify a gap, and adopt a research design that is suitable to address the gap.

For the purpose of this study, I used systematised review, which is a type of systematic review that "attempt $[s]$ to include one or more elements of the systematic review process while stopping short of claiming that the resultant output is a systematic review" (Grant \& Booth, 2009, p. 102). In general, the systematic review method has several advantages, such as clear search strategy and sources, clear focus and outcomes, and can be replicated and minimise bias (Perry \& Hammond, 2002). It was also found to enhance postgraduates' understanding of their research topics (Armitage \& Keeble-Allen, 2008). However, researchers suggest using systematised review for postgraduate students (Grant \& Booth, 2009) because typical systematic review requires more than one researcher (Daigneault et al., 2014). In addition, the scope of sources in systematic reviews is usually wide and can be time consuming and overwhelming for one researcher; hence using "less demanding but nevertheless systematic methods of reviewing" (Perry \& Hammond, 2002, p. 274) provide a suitable approach to restrict the scope. The systematised review of my study included the five most-cited and highly ranked journals to explore the tendency of the prevalent research in educational technology. Furthermore, choosing leading peer-reviewed journals is one of the methods to validate the quality of the studies (Levy \& Ellis, 2006), which is part of the systematic review process. I argue that exploring the trends of the most-cited articles in educational technology provides an indication of possible gaps that exist in the field.

\section{Selection of Resources}

To determine the most-cited and highly ranked journals in educational technology, first: I followed the Crompton et al. (2017) approach by using Google Scholar Metrics to identify the top 10 journals in educational technology which have high impact factors. Then I excluded journals that did not focus on primary education and classroom settings, such as Internet and Higher Education and The International Review of Research in Open and Distributed Learning. Second, I selected the top five journals that were ranked within quartiles Q1, which refers to journals with the highest values. This ranking was according to 
SCImago Journal \& Country Rank (SCImago, n.d.), which includes the journals' scientific indicators developed from the information contained in the Scopus ${ }^{\circledR}$ database. The rankings in both Google Scholar Metrics and the SCImago Journal \& Country Rank were retrieved and updated in December 2019. The selected journals are listed in Table 2.1, below.

Table 2.1

Selected Journals for the Systematised Review

\begin{tabular}{|c|c|c|c|c|c|}
\hline & \multirow{2}{*}{ Journal } & \multicolumn{2}{|c|}{ Google Scholar Metrics } & \multirow{2}{*}{$\begin{array}{l}\text { Scopus } \\
\text { Cite Score }\end{array}$} & \multirow{2}{*}{ SJR } \\
\hline & & h5-index & h5-median & & \\
\hline 1. & Computers \& Education & 94 & 135 & 7.72 & Q1 \\
\hline 2. & $\begin{array}{l}\text { British Journal of } \\
\text { Educational Technology }\end{array}$ & 56 & 86 & 4.07 & Q1 \\
\hline 3. & $\begin{array}{l}\text { Journal of Computer } \\
\text { Assisted Learning }\end{array}$ & 35 & 51 & 4.11 & Q1 \\
\hline 4. & $\begin{array}{l}\text { Education and Information } \\
\text { Technologies }\end{array}$ & 34 & 63 & 2.19 & Q1 \\
\hline 5. & $\begin{array}{l}\text { Educational Technology } \\
\text { Research and Development }\end{array}$ & 34 & 60 & 3.29 & Q1 \\
\hline
\end{tabular}

\section{Inclusion/Exclusion Criteria and Analysis}

The review was an aggregative (Gough et al., 2012), which means it included quantitative, qualitative, and mixed studies. The Boolean used for searching was (device* OR Chromebook OR laptop) AND (use* OR usage) AND (classroom). This term retrieved all the studies that used the terms laptops, Chromebook, devices including 'mobile devices' and 'digital devices', which usually come in conjunction. Although the Boolean retrieved a considerable number of articles that included other types of devices such as tablets, smartphones, computers, and others, it did not retrieve all the studies that only used the terms tablets, iPads, or computers to refer to the devices used. This is a limitation of this systematised review. The search included studies published between January 2015 to December 2019. The initial numbers of articles retrieved are listed in Table 2.2, below. I read the abstract of each article and saved the selected articles. Then a deeper read of the articles was conducted to ensure that the inclusion criteria listed in Table 2.3, below, were met. I developed these criteria to include the relevant studies to the aims and scope of the current study. For example, the types of devices included the common types used in New Zealand 
middle and upper-primary classrooms such as Chromebooks, laptops, tablets, and computers. This process resulted in a total of 64 articles (See Appendix A).

Table 2.2

Retrieved and Selected Numbers of Studies

\begin{tabular}{lccc}
\hline Selected Journals & Retrieved & $\begin{array}{l}\text { Selected articles } \\
\text { after reading } \\
\text { abstracts }\end{array}$ & $\begin{array}{l}\text { Selected articles after } \\
\text { scanning the article }\end{array}$ \\
\hline $\begin{array}{l}\text { Computers \& Education } \\
\text { British Journal of }\end{array}$ & 234 & 65 & 13 \\
$\begin{array}{l}\text { Educational Technology } \\
\begin{array}{l}\text { Journal of Computer } \\
\text { Assisted Learning }\end{array}\end{array}$ & 133 & 38 & 14 \\
$\begin{array}{l}\text { Education and Information } \\
\text { Technologies }\end{array}$ & 333 & 39 & 4 \\
$\begin{array}{l}\text { Educational Technology } \\
\text { Research and Development }\end{array}$ & 124 & 5 & 14 \\
\hline
\end{tabular}

Table 2.3

Inclusion and Exclusion Criteria

\begin{tabular}{|c|c|c|}
\hline & Inclusion & Exclusion \\
\hline 1 & Empirical studies. & $\begin{array}{l}\text { Viewpoint, theoretical, policy, articles were } \\
\text { excluded. }\end{array}$ \\
\hline 2 & $\begin{array}{l}\text { Primary Education between Year } 3 \text { to } \\
\text { Year } 8 \text { or equivalent. }\end{array}$ & $\begin{array}{l}\text { Early childhood, special education secondary, } \\
\text { higher \& adult education were excluded. }\end{array}$ \\
\hline 3 & Classroom setting. & $\begin{array}{l}\text { Informal settings, distance learning, online, } \\
\text { after school settings were excluded. }\end{array}$ \\
\hline 4 & $\begin{array}{l}\text { Devices included (tablets, laptops, } \\
\text { PCs). }\end{array}$ & $\begin{array}{l}\text { Devices such as smartphones, clickers, iPod, } \\
\text { multi-touch tables, etc. }\end{array}$ \\
\hline 5 & $\begin{array}{l}\text { The focus was on using digital devices } \\
\text { by students for classroom learning. }\end{array}$ & $\begin{array}{l}\text { Studies about policies, designing research } \\
\text { instruments, gaming for non-educational } \\
\text { purposes, teachers' use, or using devices as a } \\
\text { testing tool or an instrument were excluded. }\end{array}$ \\
\hline
\end{tabular}

\section{Analysis and Synthesis}

I developed a summary table using MAXQDA 2020 to analyse the articles. That included the context, participants, subject area, devices, design, methods, focus or research questions, and 
the findings. Based on the literature, I classified the design of the studies into experimental and non-experimental; methods were classified into qualitative, quantitative, and mixed. Then each article was given a symbol based on Table 2.4, below, as in the example in Figure 2.1, below. That made it easy for me to distinguish from one look the features of each study when organised in a summary table or a figure.

Table 2.4

Legend for Articles' Symbols

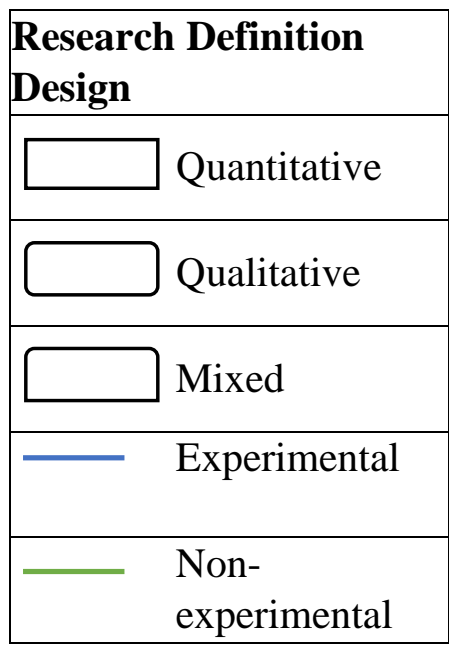

\begin{tabular}{|cl|}
\hline $\begin{array}{c}\text { Journal } \\
\text { Name }\end{array}$ & Definition \\
\hline EI & $\begin{array}{l}\text { Education and Information } \\
\text { Technologies }\end{array}$ \\
\hline CE & Computers \& Education \\
\hline BJ & $\begin{array}{l}\text { British Journal of } \\
\text { Educational Technology }\end{array}$ \\
\hline ET & $\begin{array}{l}\text { Educational Technology } \\
\text { Research and Development }\end{array}$ \\
\hline JC & $\begin{array}{l}\text { Journal of Computer } \\
\text { Assisted Learning }\end{array}$ \\
\hline
\end{tabular}

\begin{tabular}{|cl|}
\hline $\begin{array}{c}\text { Subject } \\
\text { Area }\end{array}$ & Definition \\
\hline $\mathrm{L}$ & Language \\
\hline $\mathrm{M}$ & Maths \\
\hline $\mathrm{S}$ & Science \\
\hline $\mathrm{D}$ & Digital Literacy \\
\hline $\mathrm{H}$ & Social studies \\
\hline $\mathrm{G}$ & Generic \\
\hline $\mathrm{T}$ & STEM \\
\hline $\mathrm{U}$ & Music \\
\hline $\mathrm{E}$ & Media \\
\hline
\end{tabular}

\section{Figure 2.1}

Illustration of the Study's Symbol

Bold two letters and number refer to journal name and article number (sorted alphabetically)

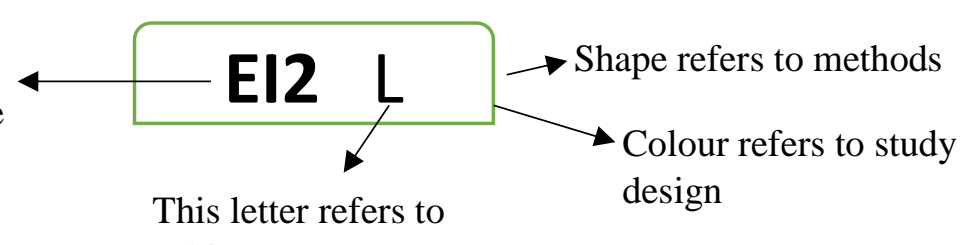
subject area

For the focus, it was inductively (Braun \& Clarke, 2006) classified into two categories:

(a) Intervention-focused studies, which refer to studies aimed to examine or evaluate the influence or impact of a certain intervention. That does not mean that all of the studies within this category were experimental interventional studies in which the research team intervened and the study is "specifically tailored to evaluate direct impacts of treatment" (Thiese, 2014, p. 199). I included non-experimental studies that aimed to explore the 
influence of a specific intervention. This category was further analysed inductively to classify the interventions. Five themes emerged: pedagogical approaches, initiatives, applications, educational games, and comparison of use conditions.

(b) Non-intervention studies included studies that did not investigate a particular intervention but focused on students' experience. Figure 2.2, below, illustrates the analysis and synthesis of the reviewed studies.

My strategy to report the findings of this review is to first describe the findings then combine my critiques and arguments with the gap discussion. This strategy enabled me to first pinpoint the gap clearly then clarify my argument that justifies the approach adopted to address that gap. 
Figure 2.2

Classification of the Studies Based on Focus
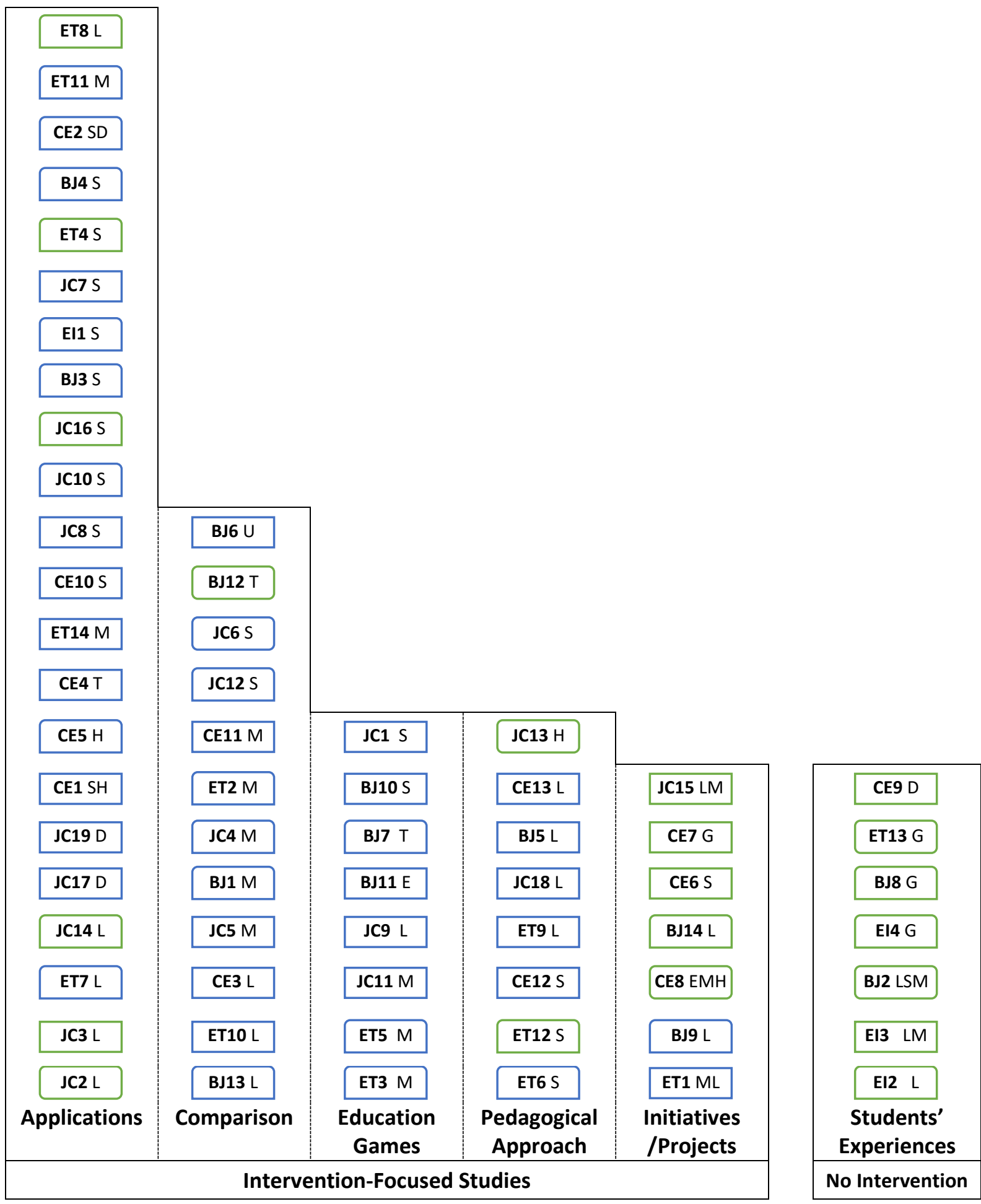


\section{Approaches and Focuses of Research on Digital Devices}

As claimed, the review shows an increase in numbers of studies on using devices in classrooms as in Figure 2.3, below, which reflects the increase of interest in studying the phenomenon. That increase can be attributed to the wide adoption of integrating devices all over the world as shown in Figure 2.4, below. Both developed and developing countries from all continents conducted research in this area favouring Asia, Europe, and North America, taking into account that some studies were conducted in more than country.

\section{Figure 2.3}

\section{Number of Reviewed Studies Published Per Year}

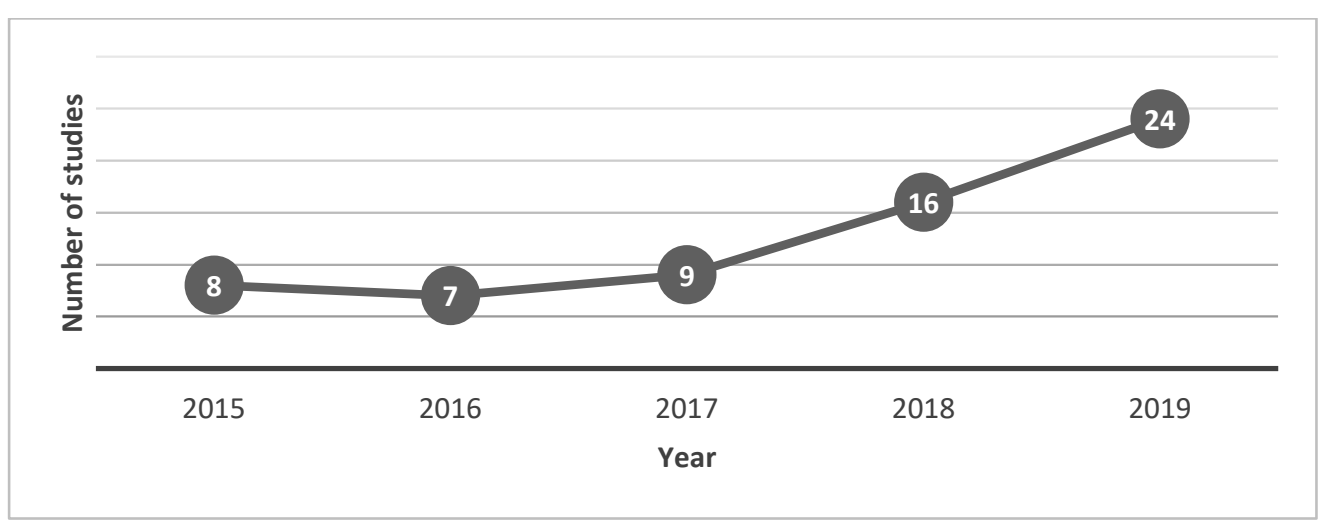

Note: Some studies published in 2019 were issued in 2020

\section{Figure 2.4}

Geographic Distribution of the Studies

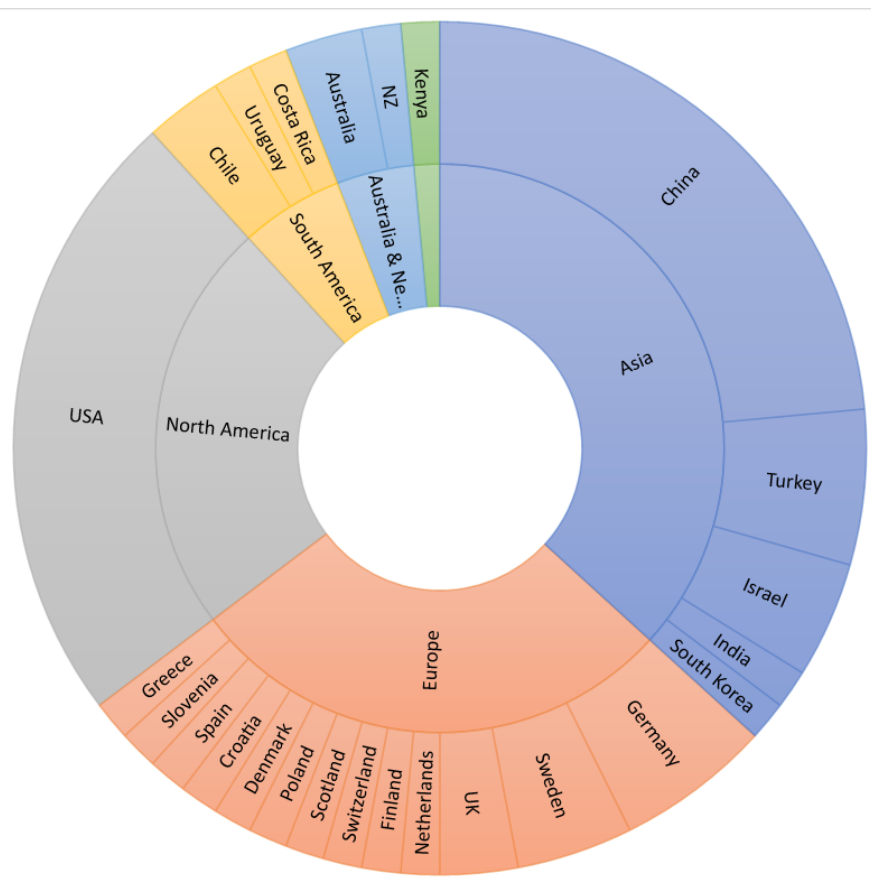


The review showed that digital devices were used for different subject areas (Figure 2.5, below). Much of the reviewed literature paid particular attention to traditional subject areas such as science, language, and maths. Fewer studies explored the use of devices for STEM and Media. Only one study in this review explored using devices for music. Some studies explored using digital devices across different subject areas and did not focus on specific ones; this category was called generic. Among the included devices in this review (Figure 2.6, below), most of the studies reported the use of tablets and iPads, followed by computers and PCs. Fewer studies reported the use of laptops, including notebooks, Chromebook, and netbooks.

\section{Figure 2.5}

Number of Studies Investigated Using Devices for Subject Areas

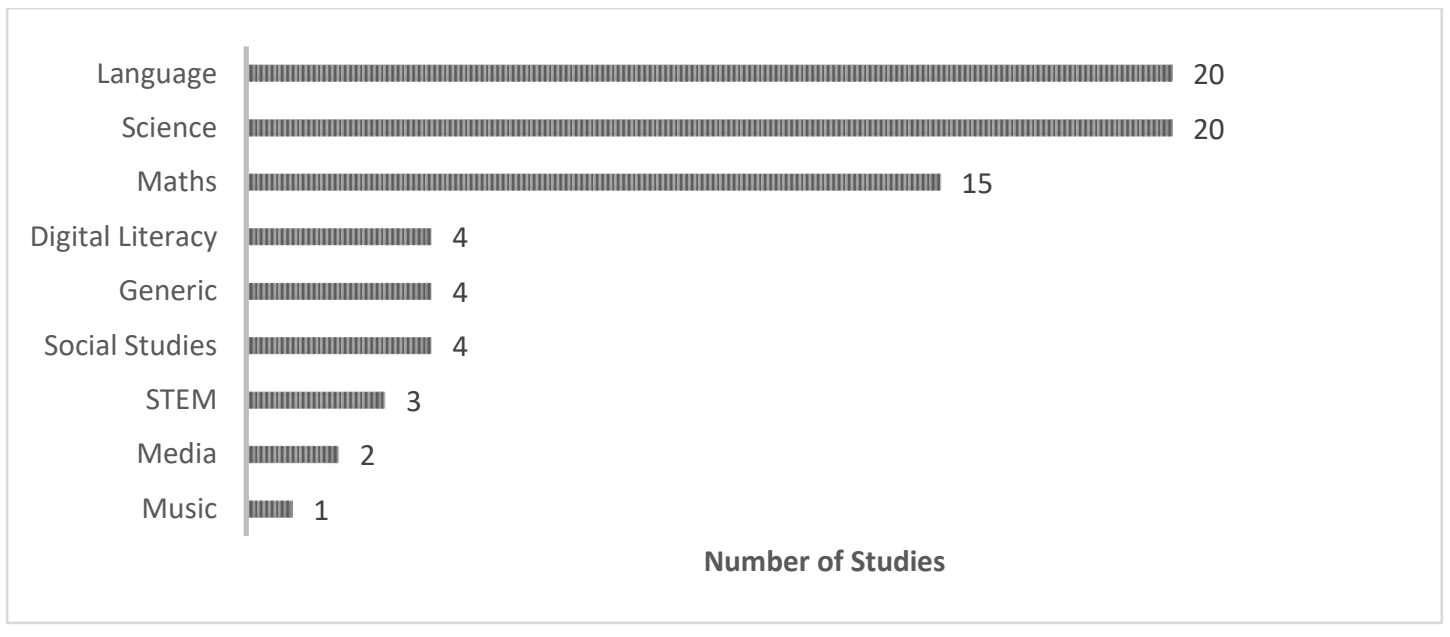

Figure 2.6

Number of Studies Reported for each Type of Included Device

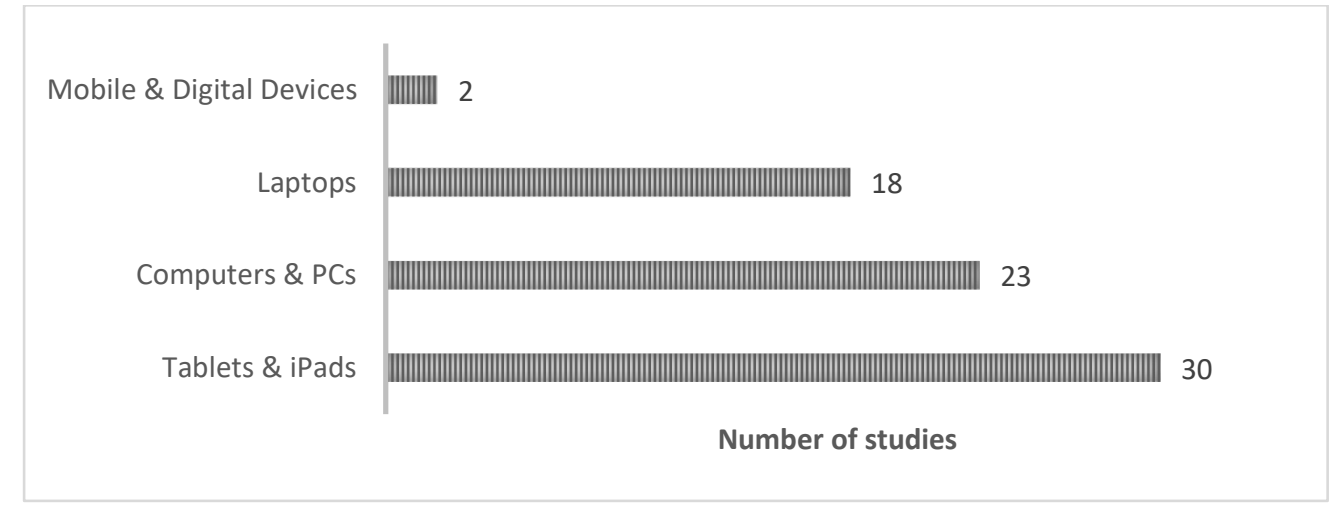

Most of the studies, about $89 \%$, were intervention-focused studies. Regarding research design, $67 \%$ of the studies were experimental. A variety of methods were used: most 
of the studies were quantitative and mixed and much fewer were qualitative. The detailed numbers are summarised in Figure 2.7, below.

In summary, using digital devices in primary classrooms is a growing research area. Reports about this phenomenon come from different formal education contexts around the globe. There is still a focus on the traditional subject areas compared to integrated subject areas such as STEM. There are more studies that reported using tablets than those that reported using other devices. Studies examining interventions in the top journals were dominant, with an apparent tendency to adopt experimental designs. Most of the methods used were quantitative and mixed methods and, to a much lesser extent, qualitative methods.

\section{Figure 2.7}

Numbers of Studies in Terms of Their Focus, Design, and Methods

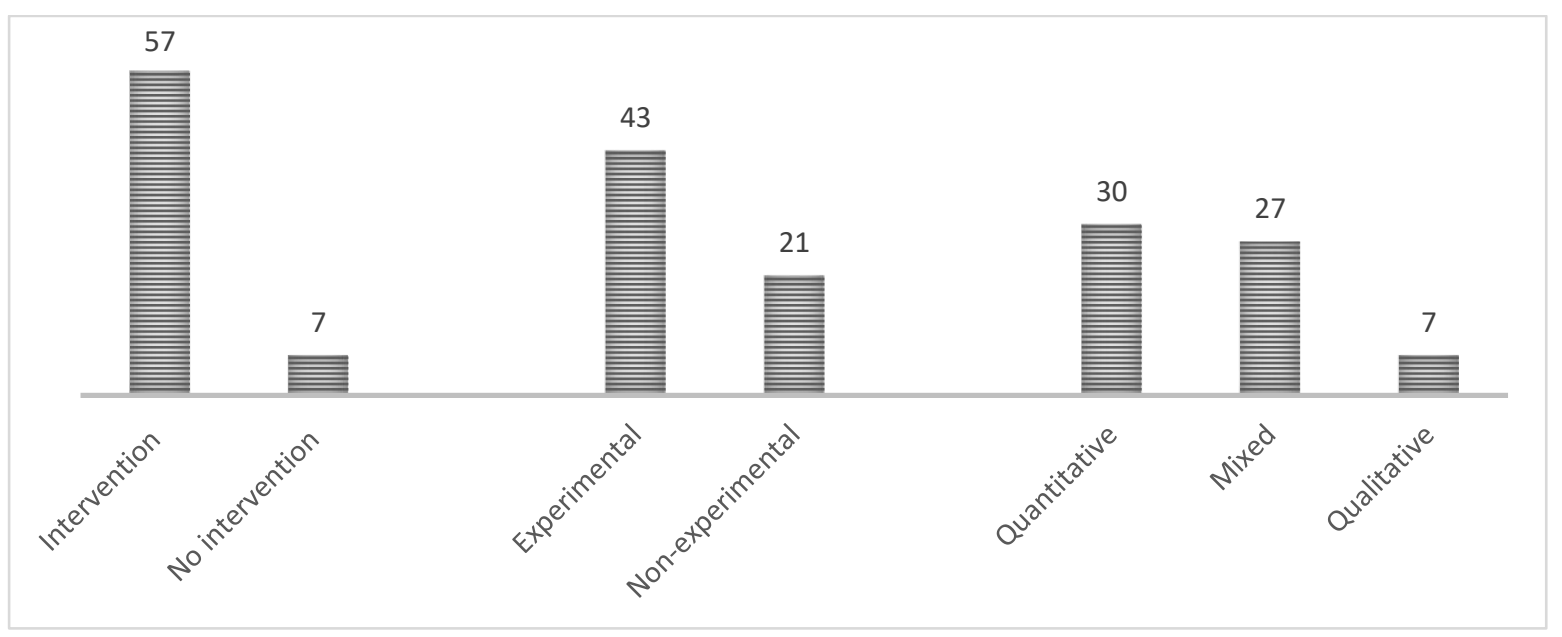

\section{Using Devices for Educational Interventions}

Most of the studies in this review aimed to examine the influence of interventions. In the next sections, I report the major findings of the studies related to the following interventions: projects and initiatives, pedagogical approaches, educational gaming, comparison to traditional approaches, and applications.

\section{Initiatives to Integrate Digital Devices in Classrooms}

Studies from different contexts reported initiatives related to integrating digital devices in classrooms. These studies included developed and developing countries, which indicates the expansion of the phenomenon globally. Implementing such initiatives in low socio-economic communities can mitigate students' performance gaps and reduce the digital divide. Kennedy et al. (2016) compared students' ability to conduct online research and learn in science in two 
states in the USA. One state implemented a one-to-one programme in schools and the other did not but had higher median family income. The results showed that students in the first state were able to perform better in performance-based assessments in digital literacy.

The pedagogical approach has been identified as a major contributing factor for the success of these initiatives. A study reported the influence of a project that took place in five schools in five European countries: Croatia, Denmark, Poland, Sweden, and the UK (Walton et al., 2019). The students were expected to create digital artefacts such as comics, videos, and animations as an activity to enhance their motivation to learn about the national literature of their countries and other countries. Students' learning, motivation, engagement, and selfefficacy were improved as creating digital artefacts allowed students to demonstrate different competencies beyond academic capabilities.

Other studies reported the importance of adopting pedagogical approaches that are sensitive to the cultural and contextual aspects. For example, a study about a contextualisedOLPC education project related to the One Laptop per Child (OLPC) initiative in India showed that contextualising the integration of digital devices resulted in positive learning outcomes (Ale et al., 2017). Another study from Kenya that examined a one-to-one tablet initiative emphasised taking cultural aspects into account when using devices in classrooms as they influence the pedagogical and technological approach (Heinrich et al., 2020). It also found that the contextual factors of the low-resource countries require creative ways of using the devices for learning in classrooms. It showed that, in some cases, shared use of the devices had a positive impact on cooperative learning and positive learning outcomes on language learning. The findings related to the shared access to devices align with a study that was conducted in New Zealand on schools that participated in a netbook purchasing scheme for children in a low socio-economic community (Starkey \& Zhong, 2019). It compared academic achievement in reading, writing, and maths for children who had a dedicated netbook for use at school and home and those who did not but only had shared access at school. The data collected from assessment tools across two years showed no significant differences in academic achievement between the two groups. It was concluded that the ownership of the devices might not be a significant factor that impacts students' achievement. However, the study reported that the pedagogical approach can decrease the achievement gap between students who can access their devices at home and school and those who are limited to shared access at school. 
The outcomes of integrating digital devices in classrooms are also influenced by factors related to social actors in the classroom environment, that is, teachers and students. Regarding teachers, a non-experimental study examined the influence of a professional development initiative on students' learning experiences in four classrooms in the USA (Mouza \& Barrett-Greenly, 2015). The study found that teachers' skills and knowledge about using mobile devices and applications were enhanced. That was reflected in students' use and achievement; students used the devices to locate information from the Internet and create digital artefacts. A positive impact on students' content learning, empowerment, and academic growth was evident. That supports the findings of a previous study that showed, at the same time, that the findings draw attention to the role of pedagogical approaches that can decrease the achievement gap between students who can access their devices at home and school and those who are limited to shared access at school. With regards to students, a tablet-based interactive classroom programme was implemented in rural schools in South Korea; using devices was explored in terms of "frequency of tablet use, usefulness, ease of use, satisfaction, the desire to learn, and self-efficacy for learning” (Kim \& Jang, 2015, p. 12). The study found that the desire to learn in a tablet-based environment is the key factor that predicts students' beliefs about the future. The positive perceptions of the use, such as usefulness and ease of use, positively influenced students' beliefs about the future and selfefficacy only for those who had a desire to learn with devices.

The findings extracted from this group of studies showed that good intentions behind providing devices for all students, such as reducing the digital divide, were not enough to guarantee positive outcomes. Factors relating to the pedagogical approach, contextual factors, teachers, and students are critical. However, while these factors usually exist together in classrooms, each study focused on a specific factor which did not illustrate the collective influence of these factors on using devices in classroom learning.

\section{Pedagogical Approaches to Interventions}

This category included studies that focused on the effectiveness of pedagogical approaches that required using digital devices without designing a particular software. This means the software used in these studies was available online. The discussion of these studies will be in terms of the subject area, as the influence of the intervention was usually measured in relation to students' achievements in each subject. 
Language learning. Most of the studies in this category explored using devices for language learning. Pedagogical approaches that integrated digital devices could positively influence students' language learning when they worked cooperatively to perform digital storytelling tasks; the students showed lower levels of anxiety as well (Liu et al., 2018). In addition, using devices can influence feedback in classrooms. For example, in essay writing, a study compared receiving teacher's feedback combined with an automated essay evaluation system available online called PEG Writing, against receiving teacher's feedback via Google Docs (Wilson \& Czik, 2016). The findings showed that, although the teachers gave the same amount of feedback in the same conditions, their feedback focused more on higher-level writing skills in the combined feedback condition. It also showed that students' motivation increased, while there were no statistically significant differences between conditions on the quality of the final draft. Another pedagogical approach that was designed as a touch-typing course and delivered by the teacher, enhanced primary students' spelling, typing speed, and narrative-writing skills (van Weerdenburg et al., 2019). However, not all pedagogical approaches that utilise online software had the same impact; a study showed that for effective and deep learning, using digital knowledge graphs as an approach for language learning was better than the common digital concept map (Cui \& Yu, 2019).

Science: For science learning, some studies showed effective pedagogies that benefit from available software online. An example was a self-regulated science inquiry approach, which was created using student engagement platform, Nearpod; that approach improved students' achievement, help-seeking, self-efficacy, and self-regulation (Lai et al., 2018). Another approach that embedded a 'productive failure instructional design' in project-based learning was found to improve deep conceptual knowledge comprehension, positive attitudes towards challenges, and autonomous learning; the approach improved students' collaborative problem solving and the quality of their digital products (Song, 2018). On the other hand, a study drew attention to the impact of emotions developed while using multimedia during learning. It found that although learning materials with positive emotional design can cause positive emotions while learning, they do not necessarily enhance learning; additionally, the entertainment caused by animated multimedia led to less mental effort in learning (Uzun \& Y1ldirım, 2018).

Social studies: one study explored how students' exploration and sense of place were shaped by using mobile technologies; the findings showed that when physical activities and digital information were integrated with learning activities to create digital artefacts, the 
students were able to develop a new sense and understanding of place and it supported students' imagination and creation of narrative in history (Price et al., 2016).

This category showed how available software could be used as part of pedagogical approaches in different subject areas. The replication of such studies could be easier in other contexts as no particular software was designed to undertake the study. However, the focus of these studies was mostly on the effectiveness of the pedagogical approach under controlled conditions, which ignores the multiple factors that might face practitioners in other contexts.

\section{Using Devices for Educational Gaming}

Special educational games were designed to support students' learning in different subject areas. Interestingly, the enjoyment was not the major factor that affected students' decision to play educational games; other factors were more influential, especially teachers' support and clear learning tasks and instructions of the game (Iten \& Petko, 2016). Other studies highlighted the positive impact of using educational digital games on students' learning. Game-based writing elevated students' interest, participation, and performance in writing (Liao et al., 2018). In science, a collaborative mobile game that uses augmented reality enhanced students' achievement in general but more so for girls, especially when designed to align with social communication (Bressler et al., 2019). The inquiry-based ubiquitous gaming approach positively impacted students' engagement and achievement in science and their perceptions of their problem-solving and critical thinking skills (Hwang \& Chen, 2017). In maths, three studies found that using digital educational games improved students' mathematical knowledge, performance, and enjoyment in classroom learning (Gresalfi et al., 2018; Ke, 2019; Masek et al., 2017). However, a study found that students' performance was not affected by gaming approach but that their engagement was enhanced (Garneli et al., 2017).

All the studies within this category were experimental; the experimental design is considered a robust evidence-based approach to inform policies and decision making (Slavin, 2002). However, in this case, difficulties arise when an attempt is made to implement or replicate these studies; these games were designed for specific contexts, curriculums, or educational systems, which limits the benefits of these games unless they were commercialised or customised. Additionally, as in the previous category, the focus of the studies was on the effectiveness of these games, so not much was reported about the students' experiences when using these devices and the factors that influenced their uses. 


\section{Using Devices in Comparison to Traditional Methods}

In this category, researchers compared between classroom learning when using devices and not using them. One study examined music learning. Although the students found it easy to use an interactive mobile application for learning solfeggio (music theory), the app had a minor impact on their singing and tapping, yet it enhanced students' musical intervals and rhythmic accuracy (Debevc et al., 2020). Other studies were related to the following subject areas:

Language learning. For language learning, using devices did not guarantee outstanding outcomes. A digital tool was developed to enhance syntactic structure learning where a student used the visual-syntactic text format to read English and social studies topics (Park et al., 2019). The findings showed that the significant gains in the English language were limited to sixth graders, not the younger students. Additionally, students who used the digital tool achieved better in the English language test than students who used printed or digital traditional textbooks. For reading, although students preferred reading from devices, their performance and confidence were significantly better when reading from paper (Dahan Golan et al., 2018). Another study explained that the dependency on digital media materials is causing fragmented reading. That leads to lower levels of comprehension and attention, especially when combined with the distraction caused by online multitasking such as checking emails, chatting, and browsing the web (Liu \& Gu, 2020).

Maths and STEM. Unlike the previous category, using devices seemed to be promising for maths and STEM subjects compared to traditional ways. A study showed that for solving arithmetic exercises, the students who received instant feedback through their personal or interpersonal computers achieved better than students who used pen-and-paper and received delayed feedback (Alcoholado et al., 2016). Another study found that teachers' orchestration between digital and non-digital resources combined with the appropriate school infrastructure to support digital learning improved students' learning in maths (Díaz et al., 2015). Additionally, students who used a computer-based story for maths problem solving achieved better than students who used a paper-based story or did not use a story (Gunbas, 2015). For STEM subjects, students who used 3D computer-aided design in a STEMintegrated approach performed significantly better in maths and developed better spatial skills than students who used traditional methods on papers ( $\mathrm{gg} \&$ Chan, 2019). However, not all desired learning outcomes could be achieved by using devices; although students who used mobile technologies in learning maths achieved better and showed more enjoyment compared 
to students who used paper and pen, no difference in attitude towards maths was found (Fabian et al., 2018). For learning fractions, mathematical discourse using blogs led to better conceptual knowledge compared to face-to-face conversation; however, there was no difference in procedural knowledge (Stoyle \& Morris, 2017).

Science. For science learning, using devices resulted in better outcomes compared to traditional methods. Students' scientific knowledge and argumentation skills and quality were significantly better when using a graph-oriented computer-assisted application in a projectbased learning environment compared to students who used traditional tools such as notetaking (Hsu et al., 2015). Students who used electronic workbooks on their computers to learn about the conventions of diagrams were more engaged and accurate in answering questions and had more improved diagrammatic reasoning than students who used printed workbooks (Miller et al., 2016).

As in the previous category, all the studies within this category were experimental except for one; in this case, the comparison could be useful to inform practice. However and based on what has been mentioned earlier, focusing on measuring and comparing the outcomes offers no further explanation of the interactions and factors that led to these outcomes.

\section{Examining Applications for Classroom Learning}

This sub-category includes the largest number of studies with the intervention-focused category. The common focus of these studies was examining the impact of an application designed for a specific intervention. As in the previous sections, studies were grouped based on subject areas.

Language learning. Researchers developed and tested various applications for language learning which showed positive learning outcomes. Some of these applications facilitated collaborative learning. A mobile learning system designed in familiar authentic environments was beneficial and the best performance happened during the tightly collaborative learning tasks (Shadiev et al., 2018). Another study attributed the positive results of a collaborative digital storytelling platform to the classroom environment that gave the student the freedom to form groups, which encouraged collaboration and engagement (Liu et al., 2019). The study found that students' participation and their oral reading proficiency were enhanced. Additionally, the features of the application and the teachers' approach in utilising them were important to make successful use of the applications. For 
example, a Fluency Tutor programme with teacher mediation, constant feedback, and a feature of text repetitions enhanced students' fluency from low-resource communities, especially those who were shy or unconfident (Lange, 2019). Some features, such as an interactive touchscreen tool, were found to increase students' engagement and motivation in reading and writing (Cordero et al., 2018). However, not all digital product features affected students' learning in the same way. For example, no difference was found in students' achievement when using animated graphics or using static graphics in a computer-based English achievement assessment (Dindar et al., 2015). Alternatively, the quality of the graphics was the crucial factor.

Science. Unlike literacy, students gained a deeper understanding of chemical reactions when animations were used compared to static graphics (Stebner et al., 2017). That could be due to the abstract nature of scientific knowledge, for which digital technologies could provide representation. For example, a remote laboratory system to conduct online scientific experiments was efficient for science learning inside and outside classrooms (Tho $\&$ Yeung, 2018) as the students were able to experience virtual interactions instead of only dealing with equations. Primary students who used a digital Electricity Exploration Tool developed a better conceptual understanding of electric circuits when learning with constantly concrete simulation elements rather than combining concrete and abstract elements (Jaakkola \& Veermans, 2015). Recently, augmented reality is gaining attention for science learning. An augmented reality system with a two-tier test strategy improved students' motivation, conceptual knowledge, and achievement in science (Chang et al., 2020). Further, students prefer using augmented reality applications individually rather than collaboratively (Baran et al., 2020). Another system developed that combined augmented reality with flipped learning was compared to the conventional flipped-learning approach; the findings showed that students who used the combined learning system had better learning achievement, motivation, student group self-efficacy, and critical thinking orientation (Chang \& Hwang, 2018).

Digital applications could enhance students' learning skills in science. When comparing using a stage-dependent argumentation assistance tool with a generic text-based interface, it was found that students' scientific argumentation and comprehension of the scientific process was enhanced when using the tool (Lin et al., 2018). Using a technologyenhanced learning environment for collaborative knowledge-building activities enhanced students' comprehension, collaboration, creativity, and independence while learning about 
energy (Hong \& Lin, 2019). Another mobile experiential learning system was developed for environmental science; it improved students' problem-solving skills, collective efficacy, attitudes, and achievement compared to students who used a conventional situated mobile learning approach (Cheng et al., 2019). At the same time, learner characteristics were found to impact the effectiveness of digital learning environments. A study found that students with low prior knowledge benefit from visual signalling and an animated pedagogical agent for science learning but they did not benefit high prior-knowledge students (Johnson et al., 2015). This presents an example of the mutual influence that could happen between the learner and the devices during learning.

Maths and STEM. What applies to abstract knowledge acquisition in science can also be applied to maths. A computer-aided design tool, called Energy3D, was effective in developing students' knowledge and use of mathematical and scientific concepts in an integrated STEM learning environment (Dasgupta et al., 2019). For spatial task solving, students who used an app that enables physically rotating 3D figures on their tablets gained higher scores compared to students who did it mentally without the rotating feature (Wetzel et al., 2020). However, in a multi-touch collaborative learning environment, an iPad application with prompts impacted learners differently; verbalisation prompts increased offtask behaviour and negative emotions, and enhanced the quality of students' dialogues but had no impact on learning gains (Schmitt \& Weinberger, 2019).

Social sciences. In the social sciences, positive outcomes resulted from using applications designed for learning. In this category, a positive impact on students' argumentative writing in science and social sciences happened when using a computer-based graphic organiser with embedded self-regulated learning strategies (Boykin et al., 2019). Another study showed that students' performance and interaction with the content were significantly better when using augmented reality and a mobile pedestrian navigation app compared to using desktop devices (Joo-Nagata et al., 2017).

Digital Literacy. For learning the logic of programming, providing digital units that included rich explicit instructions led to a significant increase in learning gains, transfer of knowledge, and motivation for learners (Witherspoon et al., 2018). Additionally, adding a summary of the main points for the digital instructions increased the effectiveness of these resources; a study found that video tutorials for software training promoted students' 
motivation, self-efficacy, and performance, especially when the task demonstrations included the reviews or the summaries (van der Meij \& van der Meij, 2016).

As in the gaming category, special applications were designed for certain interventions. These studies provide sound evidence related to the effectiveness of these interventions; however, in order to benefit from the results of these studies, the applications should be available and used in the same conditions of the intervention, which is discussed further in the gap section shortly.

\section{Summary}

The prevalent literature explored in the systematised review focused on examining interventions using digital devices in classrooms. The findings regarding the examined interventions varied; initiatives could be implemented to reduce the digital divide and enhance students' learning; however, the effectiveness of these initiatives was influenced by the pedagogical approach and social actors' characteristics. For studies that examined the interventions related to pedagogical approaches utilising online software, most of the studies reported positive outcomes and some reported no impact. Regarding educational digital gaming, almost all studies reported positive outcomes. When comparing digital tools with traditional tools, the findings varied depending on subject areas; there were mixed outcomes related to language learning, while the outcomes were positive in science and maths. Finally, most of the studies reported the effectiveness of applications designed for special interventions. In the intervention-focused category, most of the studies published in top journals tended to use experimental designs, were subject-area-oriented, and reported successful trials. This will be further discussed in the gap section of this chapter.

\section{Students' Experience When Using Digital Devices}

Unlike the studies in the previous category, studies in this category focused on the students' experiences when using devices in non-intervention settings. The other difference was that this category was less subject-area oriented than the previous one; most of the studies explored the uses for generic or more than one subject area. All of the studies were nonexperimental: two used quantitative methods, three used mixed methods, and two used qualitative methods. One study explored how students estimate their digital capabilities, while the other six studies reported how students use the devices. In this section, I present the most relevant findings of each study, then synthesise the findings of the six studies that align with the focus of the current study. 
A study addressed the gap between students' perceptions of their digital capabilities and their digital literacy (Porat et al., 2018). The study examined six skills that comprise digital literacy: (1) Photo-visual thinking: understanding visual and graphic information; (2) Reproduction skills: creating new meaning from existing digital content; (3) Branching skills: managing and constructing knowledge from non-linear digital resources; (4) Information: critically evaluating the quality and the credibility of digital resources; (5) Social-emotional skills: benefiting from online social communication safely; and (6) Real-time thinking: processing tasks and information effectively and simultaneously. The findings showed that students over-estimated their digital skills when compared to their actual performance related to the six skills. That meant that digital natives who spend a long time in the digital environment might exhibit digital overconfidence that does not necessarily reflect their actual competencies.

The findings of the next studies were related to the uses, factors, and influence of using devices in classrooms, which means that my study can be contextualised within this category. After presenting the findings of each study, I summarise the findings to show in later chapters how the current study extends our knowledge of using devices in classrooms.

A study conducted in six classes in five Swedish schools explored the relationship between the affordances of digital writing tools and student agency (Dahlström, 2019). The study found four affordances of the digital tools that influence students' agency: (1) Writeability, which allows students to write readable texts, which influenced students' language development and communication; (2) Edit-ability, which influences students' independent writing and making it easy to correct mistakes; (3) Story-telling-ability, which allows students to independently make decisions about imagining and creating their stories; and (4) Accessibility, which allows students to participate more in writing activities regardless of their writing capabilities. Accessibility to devices significantly influenced using devices for writing; more than half of the students said that they mainly used pen and paper for writing at school. They reported that although there were adequate digital devices in their classrooms, they were not allowed to use them. Therefore, many of them relied on using their devices at home. The findings showed that teaching design and how much agency was given to students were other factors that influenced using the devices for writing.

Zilka (2020) surveyed Israeli teenagers about using devices inside schools and outside schools, which makes the latter outside the scope of my study. The findings related to the 
school environment showed that students used the devices to scan documents, take pictures, record classroom lessons, and search for information. Most of the students considered digital devices helpful for their learning and for doing their homework. The majority reported that the learning environment at schools was friendly and suggested expanding the use of devices to more subject areas. They reported that the frequency of use of the devices in classrooms depended basically on the teacher, not the subject matter nor school. They added that the features of the quality of the digital devices and classroom environment affected their preferences of using the devices in schools. Students' opinions about collaborative learning were mixed and contradictory; while some appreciated collaborative learning on devices, others found it distracting, uncomfortable, and that the classroom environment did not support this kind of learning because of the crowdedness and noise. Although most of the students considered that the digital environment at school was outdated, they still preferred integrating digital technologies in their learning.

Varier et al. (2017) examined the integration of digital devices in 18 elementary-, middle-, and high-school classrooms in the USA. They explored the factors influencing the integration, the impact of using the devices on developing 21 st-century skills, and the impact on student engagement and motivation. They classified factors impacted integrating devices into six factors: learning curve and start-up issues for teachers and students, district control and teachers' agency, internet access and parental permission, device features, content area, and school level. The study found that using devices offered opportunities to develop 21stcentury learning, move towards a learner-centred environment, and provide instant formative feedback. The study provided examples of using devices based on Lei and Zhao (2008) framework, which suggests that students use devices for expression, organisation, communication, and exploration. Finally, using devices promoted students' efficiency and self-direction but there were mixed opinions about motivation. Primary students were motivated to use devices for learning, while some students reported that they were not engaged when using the devices.

The teachers' role in utilising digital devices was explored by Geer et al. (2017). They used the SAMR model, which refers to four levels of integrating digital devices in classrooms: Substitution, Augmentation, Modification, and Redefinition (Puentedura, 2009), to examine teachers' pedagogical approaches in integrating digital devices. They found that teachers did not move through SAMR model phases sequentially and they found it challenging to decide which phase the teachers were at, which was considered as a limitation 
of the model. They suggested that professional development could enhance the teacher's integration of digital devices in classrooms. However, they found that students used the devices for searching, communicating, and producing artefacts. These uses led to better collaboration and communication among the students. Additionally, students were more independent and had more authentic learning experiences when they were learning.

Some researchers focused on using devices for collaborative learning activities. Cerratto Pargman et al. (2018) argued that to understand collaborative mobile learning more deeply, it is not enough to study the impact of using the devices; it is also important to study how learning is mediated by digital devices. They conducted a study in natural settings in classrooms with no researcher intervention to study the emergence of collaborative activities in the tablet-mediated classrooms. They observed students in maths, science, and English as a foreign language classes in four Swedish schools. They found that students established five kinds of mediations when using the devices for the collaborative mobile activities: (1) pragmatic: to transform their knowledge and create their project; (2) epistemic: to understand and comprehend their learning tasks; (3) reflexive: to manage and regulate their learning tasks; (4) emotional: to emotionally experience what they do; and (5) spatial: to create common virtual spaces to collaborate. The researchers concluded that in the tablet-mediated classrooms collaborative learning is a complex activity which emerges from different factors. They argued that how students use the devices is as important as the design of the technical features of the digital environment. They emphasised the influence of the teacher's role and instructions on utilising the devices by the students.

The need for models that help to understand the classroom dynamics when using digital devices was emphasised by Tirado-Morueta et al. (2020). The researchers argued that to promote students' academic engagement in a one-to-one environment, three psychological needs should be satisfied: relatedness, autonomy, and competence. Based on that assumption they developed a model to understand the mechanisms that promote students' engagement with regards to behaviour, emotional, cognitive, and social involvement in one-to-one mobile programs. Then they tested their model using quantitative data collected from language and maths courses. The findings emphasised the mediation role of an authentic learning pedagogical approach. The authentic learning activities in a one-to-one mobile environment were what satisfied students' psychological needs, especially the activities that were collaborative with formative teacher feedback. In addition, the type of activity and the course 
affect students' engagement. In maths, less-structured activities with teachers' coaching, scaffolding, and modelling improved students' engagement more than structured activities.

The relevant findings of the studies within this category are synthesised according to the three foci of the current study, which are the uses, the factors shaping the uses, and the contribution of the uses to students' learning in Table 2.5, below. In sum, some studies reported the observed uses without conceptualisation (Geer et al., 2017; Zilka, 2020). Others reported the uses for specific functions such as writing (Dahlström, 2019) and mediation (Cerratto Pargman et al., 2018). One study classified the uses based on a constructivist point of view (Varier et al., 2017). The studies reported some factors related to the use and the outcomes as well. Despite the rich knowledge provided by this type of research, there is a need for a model that conceptualises students' use of digital devices and answers questions related to the factors that shape them and how they contribute to the educational process in classrooms, as will be argued in the next section. 
Table 2.5

Summary of Research on Students' Use of Digital Devices

\begin{tabular}{|c|c|c|c|}
\hline Study & $\begin{array}{l}\text { Findings related to } \\
\text { uses }\end{array}$ & $\begin{array}{l}\text { Findings related to } \\
\text { factors influencing using } \\
\text { digital devices }\end{array}$ & $\begin{array}{l}\text { Findings related to the } \\
\text { contribution of using } \\
\text { digital devices }\end{array}$ \\
\hline $\begin{array}{l}\text { Dahlström } \\
\text { (2019) }\end{array}$ & $\begin{array}{l}\text { For writing: } \\
\text { *Write-ability } \\
\text { *Edit-ability } \\
\text { *Story-telling- } \\
\text { ability } \\
\text { *Accessibility }\end{array}$ & $\begin{array}{l}\text { *Access to digital devices } \\
\text { *Teaching design and } \\
\text { giving agency to students }\end{array}$ & $\begin{array}{l}\text { *Language development } \\
\text { and communication } \\
\text { *Agency and autonomy } \\
\text { *Independent decision- } \\
\text { making } \\
\text { *Participation and } \\
\text { independent judgement }\end{array}$ \\
\hline $\begin{array}{l}\text { Zilka } \\
(2020)\end{array}$ & $\begin{array}{l}\text { *Scanning } \\
\text { *Taking pictures } \\
\text { *Recording } \\
\text { *Searching }\end{array}$ & $\begin{array}{l}* \text { Teachers } \\
* \text { Subject area } \\
* \text { The quality of the device } \\
* \text { Classroom environment }\end{array}$ & $\begin{array}{l}\text { *Useful for doing } \\
\text { homework and facilitating } \\
\text { learning } \\
\text { *Conflicting views on } \\
\text { collaborative use }\end{array}$ \\
\hline $\begin{array}{l}\text { Varier et } \\
\text { al. }(2017)\end{array}$ & $\begin{array}{l}\text { *Expression } \\
\text { *Organisation } \\
\text { *Communication } \\
\text { *Exploration }\end{array}$ & $\begin{array}{l}\text { *Learning curve } \\
\text { *District control } \\
\text { *Internet access } \\
\text { *Device features } \\
\text { *Content area } \\
\text { *School level }\end{array}$ & $\begin{array}{l}\text { *Efficiency and students' } \\
\text { self-direction } \\
* \text { Motivation }\end{array}$ \\
\hline $\begin{array}{l}\text { Geer et al. } \\
\text { (2017) }\end{array}$ & $\begin{array}{l}\text { *Browsing } \\
\text { *Using educational } \\
\text { apps } \\
\text { *Photo and video } \\
\text { taking and editing } \\
\text { *Reading } \\
\text { *Emailing } \\
\text { *Watching } \\
\text { *Social networking } \\
\text { *Playing }\end{array}$ & $\begin{array}{l}\text { * Teacher's pedagogy and } \\
\text { implementation } \\
\text { *Professional programmes } \\
\text { for teachers } \\
\text { *Features of the devices }\end{array}$ & $\begin{array}{l}\text { *Collaboration } \\
\text { *Communication } \\
\text { *Authentic learning } \\
\text { *Students' autonomy } \\
\text { *Self-reliance/ } \\
\text { autonomy and } \\
\text { authenticity }\end{array}$ \\
\hline $\begin{array}{l}\text { Cerratto } \\
\text { Pargman } \\
\text { et al. } \\
\text { (2018) }\end{array}$ & $\begin{array}{l}\text { *Pragmatic } \\
\text { *Epistemic } \\
\text { *Reflexive } \\
\text { *Emotional } \\
\text { *Spatial }\end{array}$ & & Collaborative learning \\
\hline $\begin{array}{l}\text { Tirado- } \\
\text { Morueta } \\
\text { et al. } \\
(2020)\end{array}$ & & $\begin{array}{l}\text { *Authentic learning } \\
\text { activity design } \\
\text { *Type of activity } \\
\text { (structured/open) } \\
\text { *Type of course } \\
\text { (maths/language) }\end{array}$ & $\begin{array}{l}\text { *Academic engagement } \\
\text { *Students' psychological } \\
\text { needs for autonomy and } \\
\text { competence (self- } \\
\text { efficacy) }\end{array}$ \\
\hline
\end{tabular}




\section{The Gap: A Critique of the Reviewed Literature}

The review showed that the majority of research published in the most-cited and highly ranked journals in educational technology has focused on the effectiveness of interventions utilising devices in experimental or quasi-experimental settings and most of them reported successful trials. This direction has been in the field of education for more than 20 years (Slavin, 2002) and it is not limited to studying using digital devices but extends to other research interests and topics (Biesta, 2015). Biesta (2007) discusses the tendency of educational research to conduct experimental trials to offer evidence-based practices. He explains that this idea originated from the 'causal model of professional actions' which seeks to answer the question of 'what works' by looking at the intervention as a cause and the outcomes as effects. He points out that the causality model was originally imported from medical research, which granted a privilege to randomised controlled treatments; that can explain the predominance of the approach in the top journals in educational technology. He argues that this model does not fit with the educational contexts, as "being a student is not an illness just as teaching is not a cure" (Biesta, 2007, p. 57). The tendency to focus on the experimental settings led to ignoring the open and recursive nature of the educational systems (Biesta, 2010). The approach used to study a plant in a closed laboratory environment and controlling the conditions of its growth does not fit in studying a plant in a forest.

The roots of the causality model came from the reductionist analytical approach, which dominated scientific research for decades and was used to study learning processes. In this approach, learning is broken into elements where each is examined in isolation of other elements, assuming that each element has inherited, linear, and constant effects on the learning process (Ennis, 1992). For example, many studies were designed to integrate digital devices in an innovative instruction setting, then measured the learning outcomes as a direct effect of this innovation. This approach overlooks the mutual interactions that happen between elements that constantly change each other, which make these interventions, despite their merits, succeed in some contexts and fail in others. Additionally, most of the published trials, as in this review, tend to be the successful ones, which limit our knowledge about the factors that might cause their failures.

The reductionist approach generated what can be called 'static' learning models, which focus on some 'snapshots' of the learning process such as grades, examinations, or learning outcomes without investigating what elements and dynamics influenced their emergence (Hurford, 2010). This is common in research that explores the use of digital 
devices for learning (e.g., Cavus \& Ibrahim, 2017; Zacharia et al., 2016). In the review, the few studies that reported students' experience lacked conceptualisation and modelling, perhaps because it was not the focus of the research. Furthermore, this approach holds individualistic perspectives about learning by focusing on the learner, not the learning system (Hurford, 2010). Davis and Sumara (2010) argue that it is not just that "learning is complex", but also "complex systems are learning systems" (p. 856). This can be understood as not only the learner who changes during the learning process but also the whole learning system. This approach marginalised the role of the material (e.g. tools, devices, building, furniture) in shaping human learning as Fenwick (2010) claims. She suggests that learning environments are overstuffed with materials which create a mutual influence between human and nonhuman entities. She argues that these interactions are not studied sufficiently and exploring them would uncover ambiguous aspects about learning processes. In general, limited literature has addressed and described in depth how materials influence classroom learning, and what type of mutual interactions happen in a detailed way (e.g. Roth, 1996).

Furthermore, the focus on experimental trials has led to less focus on exploring the everyday experiences of the social actors. Fenwick and Edwards (2010) argue that learning is understood and valued in different contexts in different ways. Latour (1999) refutes the idea of neglecting how people conceptualise and construct their definitions about their lives. This is supported by notable social scholars who assert that social theories should be derived from social actors' accounts (Blaikie, 2007). To understand the role of digital devices in classroom learning processes it is crucial to acknowledge how learning is perceived by social actors in each context because that will affect the learning goals and how they will be achieved.

In conclusion, there are unarguable benefits of using the predominant approach to study the role of digital devices in classroom learning. It provided rich knowledge about the effectiveness of interventions that utilised devices in classrooms. However, my study argues that there is a need for in-depth investigations of using devices by students that consider the open nature of the classroom contexts, what factors shape them, and how they could contribute to classroom learning objectives. I argue that one of the reasons for this gap is not studying the phenomenon from a holistic perspective. Next, I introduce the theoretical framework of my study and the main assumptions adopted to undertake the research. 


\section{Studying the Socio-materiality of Digital Devices in Classroom Learning}

Socio-materiality is an emerging approach to study how materials and humans interact together and what emerges from these interactions to constitute educational phenomena such as learning and teaching (Fenwick et al., 2015). It is argued that by tracking human-material interactions in educational contexts, blind spots about the role of materials, such as digital devices, in reforming and reshaping educational phenomena could be highlighted (Sørensen, 2009). Fenwick et al. (2015) propose four approaches that can be used to study sociomateriality in educational contexts: Complexity Theory, Actor-Network Theory (ANT), cultural-historical activity theory (CHAT), and spatiality theories. My study adopted Complexity Theory to underpin the theoretical stance of the research that moves from focusing on causality and effectiveness towards complexity and purpose (Biesta, 2015). Assumptions from ANT were also used to support how the phenomenon will be studied and understood, as discussed in the next sections.

\section{Complexity Theory}

Different scholars have considered Complexity Theory as a separate paradigm, which has its distinct ontological and epistemological stances (Blaikie, 2007; Cohen et al., 2017; Patton, 2015). Prominent theories that challenged the dominant paradigm of the scientific enquiry such as Evolution Theory, Chaos Theory, and Systems Theory interacted together, shaping the emergence of Complexity Theory (Boulton et al., 2015) or what some scholars prefer to name Complexity science or Complexity thinking (Davis \& Simmt, 2003; Davis \& Sumara, 2006). Some scholars consider that Complexity Theory aligns with postmodernism (Cilliers, 2002), and others argue that it aligns with critical realism (Cochran-Smith et al., 2014). My position in this research is that it has its own point of view about reality.

Kuhn (2007) identifies the ontological and epistemological assumptions of Complexity Theory. With regard to the ontological question of "What is reality", she states that "Reality is dynamic, self-organising, and emergent. It is both singular and multiple at the same time and although 'it' may be studied from various perspectives the act of study will affect the 'reality' observed" (Kuhn, 2007, p. 172). This is reflected in the epistemological position, which addresses the question of 'how do we know what we know?' by considering that the knower, the known, and the relationship between them are dynamic, self-organising, and emerging as well. The concepts 'dynamic', 'self-organising', and 'emergent' will be clarified shortly. 
Complexity Theory focuses on studying complex phenomena or complex systems (Cohen et al., 2017). A complex system "comprises many unique elements that interact in multiple ways. The elements themselves can change, learn, and adapt. The connections can change, loosen, reform, and the boundaries of the system can also shift over time" (Boulton et al., 2015, p. 35). Scholars from different disciplines discuss the characteristics of complex systems. The characteristics suggested by scholars of complex systems are influenced by the nature of the natural and social sciences, although the fundamentals are convergent. For example, Patton (2011) views were influenced by evaluation studies, while Mitchell (2009) views were influenced by genetic algorithms and computer science studies. Therefore, it is important to consider the nature of the social complex systems when studying educational phenomena (Wang, 2019) as what applies to natural complex systems does not necessarily apply to social complex systems. Yet, it is required to illustrate how the theoretical framework applies to a phenomenon (Patton, 2015).

In this study, classrooms are studied as complex systems nested within other complex systems such as the school system and educational system. This opinion was posited by some scholars such as Fenwick et al. (2015) and Davis and Sumara (2006) but without detailed verifications. Hurford (2010) applied Complexity Theory perspectives and characteristics to classroom learning using perspectives coming from genetic algorithms and computer science (Holland, 1995), mathematical modelling (Casti, 1995), and biological systems (Camazine, 2001). Recent work developed by Boulton et al. (2015) posits seven characteristics that encompass the basic features of any complex system that can be applied to myriad phenomena including educational ones. In the next section, I apply the seven characteristics to the classroom context to establish my argument that Complexity Theory provides a relevant and beneficial lens to understand the educational phenomena happening in classrooms.

\section{A Complex System is 'Systemic and Synergistic'}

Systemic - not to be confused with systematic - means that the elements in a complex system are interconnected synergistically. The synergistic nature of the relationships means that the elements interact with respect to each other; as a result, what happens to any element will somehow affect the whole system. It implies that changes happen as a result of synergistic causes, not merely a single cause (Boulton et al., 2015). This nonlinear view challenges the linear assumption that attributes changes to simple, direct cause-effect relations, which can be 
predicted and controlled; it rather asserts that unpredictable, significant changes can also happen as a result of small synergistic actions (Patton, 2011).

In the classroom, many different elements interact together synergistically. When these elements interact together to achieve common goals or achievements, any change or simple event can influence the outcomes in an unpredictable way. A simple linear causeeffect explanation cannot predict the changes that might happen to the teaching, learning, or other aspects when a new element, such as a new device or material, joins the classroom. This is because the outcomes will depend on the nature of the relationships in the classroom and to what extent they are synergistic and interconnected. This feature can explain why some innovations or interventions that were evident in 'experimental research' did not give the same good results when implemented in a different context. When this feature is considered, researchers and policymakers will be more realistic about the expectations and more cautious about the claims promoting innovations and will be more ready to expect unpredictable outcomes.

\section{The Complex System is 'Multi-Scalar'}

A complex system is influenced by multi-scalar factors (Boulton et al., 2015). A new curriculum or a policy that is imposed nationwide is a large-scale factor, where a teaching strategy in a rural classroom is a small-scale factor. Yet, these two multi-scalar factors affect learning in classrooms and are also subject to change due to negative or positive feedback. In general, small-scale factors can be modified faster and more easily than large-scale factors. Complex systems are nested systems (Fenwick et al., 2015), which means that classrooms are nested within other systems such as the school system, or intersects with other systems such as the Internet. This can be a critical challenge when studying a specific phenomenon and making decisions about the boundaries of the research; in other words, what scales to include and what not to. Research questions are important for drawing the boundaries around the research (Boulton et al., 2015) and using a case study method is another useful approach to determine what scales to include (Blaikie, 2009). In general, it is not required, or possible, to include all the scales and factors that affect a complex system in research, but it is possible and required to acknowledge that the complex phenomenon under study is influenced by multi-scalar factors. 


\section{Resilience and Adaptability Results from Diversity, Variation, and Fluctuations}

In classroom settings, there is macro-diversity which means there are a variety of elements that compose the classroom, for example, students, teachers, devices, books. Also, there is micro-diversity, which is the variety within the same type of elements such as different students, different teachers, different devices. Fluctuations are more relevant to the 'diffuse average factors' such as the changing number of students every week or the changing budget of the classroom every term. The absence of diversity, variation, and fluctuations means there will be minimal options, elements, or interactions and will create rigid laws that govern the complex heterogeneous system. The result is a weakness of the system's ability to face challenges, or emerge, and may lead to its collapse at the end (Boulton et al., 2015).

Applying these features to classrooms could be controversial. It can be argued that many educational systems, like the one I came from (i.e., Palestine), are very rigid with a tendency to curb diversity. This is not the case with respect to the educational system in New Zealand, where schools have the autonomy to self-organise themselves, and develop their own policies and curriculum to meet the needs of their local communities (Starkey \& Finger, 2018). I had the chance to visit many schools and classrooms in New Zealand and was able to address the diversity, variation, and fluctuations at all levels starting from students' cultures and backgrounds, teaching strategies, school policies, and classroom curriculum, to the outcomes. That makes studying the educational system in New Zealand using Complexity Theory of much value. It is worth noting that the impact of these qualities on the resilience and adaptability of the educational system in New Zealand is a gap worth studying in future research.

\section{Context, Events, and History Shape the Future}

The destiny and what emerges in a complex system are not just a result of elements that interact together, as said before, but also the context where all the events happen and the order of these events - the history - are key factors that shape the future (Boulton et al., 2015). The outcomes of learning activity in a classroom are not guaranteed; the order of the dynamic and where it happens in the classroom can help us understand why even when all the elements are present and interact together we get a different result. Many teachers and educators assert the importance of classroom management, organising the sequence of the flow of the classroom activities and the circumstances surrounding the classroom (Emmer \& Stough, 2001). 


\section{Change in Complex System is Episodic}

As mentioned in a previous point, complex systems are resilient because of their diversity and fluctuations, but there are tipping points that can shift and change the whole system into a different new system that have different characteristics due to the changes in the patterns of interactions. Generally, it is hard to predict whether the situation is going through tipping point or not; adequate time is needed before grasping that a tipping point has happened and changed the dynamics and the norms of the complex system. The impact and the changes that happen due to tipping points are unpredictable, and the outcomes are ambiguous. A widespread common sense implies that changes happen because of constant incremental efforts; interestingly, in most cases, this type of constant change keeps the system stable and able to hold its own overall features unchanged. The radical change is episodic; in other words, a 'revolutionary' tipping point is what makes a significant change in the system (Boulton et al., 2015).

In classroom settings, a new teacher might make changes with no significant changes in the school system as a whole. But a tipping point might happen when a new teacher, having a revolutionary approach, makes significant efforts that change teaching styles, relationships with other teachers and students, or students' learning. Another tipping point could be integrating interactive whiteboards that change the teaching patterns, the interactions between the students and the teacher, or the learning outcomes. Tipping points could be new policies, innovations, accidents, or initiatives. On the other hand, integrating new technology or pedagogy is unlikely to be developed into a tipping point because the revolutionary conditions are not ready yet in the educational context. In this research, I did not expect to capture a tipping point because the impact of tipping points usually takes time. However, I included in data collection methods some questions to encourage participants to report events that could be tipping points.

\section{More than One Future}

To explain this point, Boulton et al. (2015) discuss an example of 'text messaging' technology in the UK. The social shifts that happened as a result of the popularity of this technology among adolescents were unpredictable and unplanned. The interesting part was that the new patterns of communications, parenting, and marketing that appeared in the UK were not transferable to other contexts. This was because the elements and their interactions in the UK context were different from other settings, which made more than one future possible. Applying this to the context of the current study, the outcomes that result from 
using digital technologies in New Zealand classrooms are not just unguaranteed but are also not necessarily replicable in other educational systems or settings. Here is where Complexity Theory contributes to our understanding of any phenomenon; it draws attention to the conditions, interactions, and elements that create what emerges in a system (McDaniel et al., 2003).

\section{Self-Organisation, Self-regulation, and Emergence in Complex System}

Self-organisation is the process that happens after a chaos phase or after a tipping point that changes the system radically, so the elements or the agents organise themselves and create stable patterns of relationships depending on synergistic efforts and continuous feedback. These resulting patterns are not always beneficial or effective; sometimes the selforganisation process leads to fruitless patterns and sometimes does not (Boulton et al., 2015). A source of confusion can arise from the idea that self-organisation should not happen as a result of external forces (Mitchell, 2009). Boulton et al. (2015) clarify that in human systems there are usually leadership elements that can be misunderstood as external forces. They argue that these controlling elements are parts of the self-organisation process which contribute to pattern development. Introducing innovative instruction with new technology into a classroom, for example, can be a tipping point that requires from the teacher, students, and parents synergistic efforts to reorganise or establish new teaching, learning, or assessment patterns. This might change the whole classroom dynamics radically, creating what can be called a 'digital learning environment'. These efforts are not always efficient, but the process of trying to create the new patterns and adapting to the new situation is called self-organisation regardless of the effectiveness or usefulness of the outcomes.

After self-organisation efforts create new patterns of interactions, the efforts and the attempts to keep these patterns stable for a while are called self-regulation. During the selfregulation processes, there will be diversity, variety, and fluctuations that keep the system resilient and adaptive but will not change the major features of the system (Boulton et al., 2015). For example, all the efforts to keep the features of the digital learning environment or to enhance it, such as new teaching approaches, new devices, or feedback, will remain selfregulation efforts as long as the main characteristics of that classroom are stable as a 'digital learning environment'.

Emergence seems to be the most challenging concept. Patton (2015) defines emergence as "Pattern from self-organisation among interacting agents" (p. 147). But 
Boulton et al. (2015) argue that emergence is when "the final state is radically and qualitatively different from the starting state" (p. 46). Synthesising these two perspectives, emergence is the result of self-organisation processes that lead to the creation of a system holding new features and characteristics. From my review, there is still a gap in conceptualising emergence in the social systems compared to the advances in the biological systems, for example. Some scholars consider learning as an 'emergence' and provide a theoretical conceptualisation for that (Davis \& Sumara, 2006; Fenwick et al., 2015; Jacobson et al., 2016). But still, there are many unanswered questions related to learning as an emergent process that need more empirical efforts from researchers.

Cilliers (1998) clarifies that "A complex system is not constituted merely by the sum of its components, but also by the intricate relationships between these components. In 'cutting up' a system, the analytical method destroys what it seeks to understand" (p. 2). Therefore, this study adopted this holistic approach, to extend our knowledge, which was dominated by the reductionist approach, to understand using devices in classroom learning.

\section{Actor-Network Theory}

The main theorists of Actor-Network Theory (ANT) have considered ANT an approach, toolkit, sensibility, way, or method of how to study and describe phenomena (Latour, 2005; Law, 2009). ANT emphasises the how question (Law, 2009), which means it focuses on describing how a phenomenon develops, emerges, succeeds or fails. ANT avoids predefinitions, predistinctions, or judgements, leaving this task to the phenomenon to express itself (Latour, 1999), which also gives importance to social actors' accounts. Being a descriptive method rather than an explanatory theory, it is crucial to keep in mind when planning research because this means or explains the importance of combining ANT with other theoretical frameworks if the focus of the research goes beyond the descriptive goal.

ANT assumes that everything (e.g., learning, book, building, theory) is a result of relations between heterogeneous actors in a network (Law, 2002). Actors are different types of entities that could be human or non-human and have the agency to establish relations with each other to form a network (Callon, 2001; Latour, 2005). The accumulation of these relations keeps the network stable and able to extend, gives each actor in the network special features and qualities, and creates or generates an effect or a product (Fenwick \& Edwards, 2010). Hence, ANT's focus is to explore and describe how networks function (Law, 1999). 
The next sections discuss three principles, elicited from ANT, that were employed in my research: when to study the phenomena, the relationship between actors and networks, and how to study the actors. I explain each principle, give examples of how it has been employed by other researchers, and how it serves my research.

\section{Studying Phenomena in Action}

In ANT's sensibility, knowledge emerges through and within the practice. Therefore, it aims at describing a phenomenon during the formation process not describing a well-done innovation, or, in Latour's (1987) words, "We study science in action and not ready-made science or technology “ (p. 257). This orientation was influenced by Kuhn’s (1962) argumentation that practical activities performed within an efficient paradigm, not theoretical knowledge per se, are what actually produce and legitimise the scientific knowledge. Thus, Law (2009) considers ANT to be a methodology that relies on 'exemplary case studies'. Hence, investigating the process will not just reveal how actor-networks succeed but also how they fail.

Actor-networks that were investigated by ANT researchers had varied greatly. In her doctoral study, Luck (2008) investigated the installation process of interactive videoconferencing (IVC) in Central Queensland University, focusing on how instructors have employed this new technology in teaching. She showed the minute obstacles, negotiations, and enactments that affected both the success and failure of the installation and implementation of IVC in teaching practices. A different uptake to ANT was Stenliden's (2014) doctoral research, which aimed to explain the adaptation process of visual storytelling techniques in a Swedish primary school from the socio-cultural perspective of the human action. Also, it aimed to explore how social and material actions, simultaneously, affect the emergence of learning conditions relying on ANT perspectives. Here, although the researcher did not adopt an ANT rationale completely, ANT was considered and used as a suitable approach to investigate and depict the tangled relations between humans and technology in shaping learning.

This principle was used in my research to decide what was to be observed, that is, students using digital devices during learning activities. In addition, it was used to decide data collection methods which were not limited to social actors reporting or artefacts, but included methods that record their interactions with the devices. 


\section{Actor, Network, and Results are Inseparable}

Inseparable means that relationships in a network are what give each actor within the network its identity, features, and uniqueness; at the same time, the network will not be established or durable without its actors (Callon, 1987). This is why there is a hyphen (-) positioned between the words 'actor' and 'network' (i.e., actor-network) to emphasise the simultaneous mutual effects between the network and its actors (Latour, 1999). This entails that anything resulting from this actor-network is also characterised by it, and any change in these relations would cause a change in actors, networks, and outcomes. This principle was inspired by a semiotic stance that states that the meaning of any sign is a result of its relations with other signs, which urges us to consider ANT a 'martial-semiotic method' (Law, 2009).

This principle was illustrated by Nespor (1994) as cited in Usher and Edwards (2007) that although students in physics programmes and students in management programmes are both identified as 'learners' in the same university, their identities as learners are significantly differentiated. The influence and enactment of the learner in the management actor-network are much stronger and effective in shaping learning than the learner of the physics actornetwork. In another study, Habib and Wittek (2007) noticed that the implementation of portfolios in learning varied significantly from the role it was originally designed for. They suggest that ANT combined with another two socio-cultural approaches provide an empirical framework that enables profound insight into the role of portfolios and their potential as actors in higher education.

This principle aligns with the first characteristic of a complex system, which states that a complex system is systemic and synergistic. This principle influenced the analysis process and the understanding of social actors' accounts and the role of digital devices in classroom learning.

\section{Materials as Actors}

This could be the most central principle in ANT, nonetheless, the most controversial. All the entities that form a network are considered actors. There should not be distinction or discrimination in the analytical sense between, for example, human/non-human, social/natural, as all shall be treated in a symmetric manner since all the actors have agency (Latour, 2005) and impose an act on each other and shape the actor-network as a whole (Callon, 2001). This is why some researchers avoid using the human-related term 'actor', preferring the unbiased term 'actant' (Luck, 2008), which gradually dissolved in favour of 
the 'actor' term to represent both human and non-human actors (Fenwick \& Edwards, 2010). Furthermore, Law and Callon (1988) argue that dualisms and features used to classify things (e.g. social/technical) are artificially based on their work, which shows how these features are entangled and intertwined in an immanent way. Moreover, there should not be predetermined judgements or rankings about the actors and their roles, for example, true/false, large/small, strong/weak, since these labels are a result of the relations in the network, not a cause and are expected to be uncovered during the research process, not before it. Based on the previous principle, there are no inherited features or talents isolated from the actor-network that reveal them and any change in the relations may dramatically change these features and abilities (Law, 1999).

Emphasising the role and agency of non-humans actors did not pass without objections and critiques (Sayes, 2014). Researchers who adopted the ANT position provided empirical evidence to support this assumption. Law (1984) showed that not only did trained humans enable Portugal to control the very faraway land 'India', but also the role of nonhumans such as ships, winds, documents, guns, and currents were crucial for the success of that endeavour. In the educational context, Waltz (2006) illustrated how history textbooks could affect learning, teaching, and policies related to students, teachers, and schools. In his critical article, Sayes (2014) classified actors as humans, non-humans (e.g. animals, devices, natural phenomena, texts), and other types (e.g. symbolic and supernatural entities). He then argues that the agency of non-humans may affect building human societies (e.g. computers, invoices, air pumps); play the role of mediators, not just as a tool; be a part of moral and political associations; and act as a gathering of experiences, efforts, and other actors. But ANT still lacks a strong definition of agency, suggesting that the standard measure of agency is supposed to be the capability of influence not human. On the other hand, Luck (2013) mentions that some researchers have clarified that the agency of humans is driven by 'intentions', while the agency of non-humans does not, or what Biesta (2015) calls "reflexive intentional agents" (p. 207). She argues that taking the unintentional non-humans' agency into account provides a promising framework to study the roles of innovative technologies in education.

This principle asserts the role of the material in shaping the whole network or system. It justifies the focus on the relationship between students and their digital devices. This principle helped me to maintain this focus whenever a distraction occurred due to the many intertwined actors that composed the context. 


\section{The Functions of Education}

This study adopts Biesta's (2009) framework to study the educational purposes of classroom learning activities and how using devices contributes to achieving these purposes. The framework classifies the functions of education into three domains. First, the qualification functions, which are to provide learners with "knowledge, skills and understanding and often also with the dispositions and forms of judgement that allow them to "do something'" (Biesta, 2009, p. 39). Second, is the socialisation function, which means preparing individuals to be members of the wider community. In opposite to the second function, the third function, subjectification, is to develop the autonomy and the independence of the learner when they think or act. Biesta argues that these three functions are intertwined and interconnected in an inseparable manner. The three functions influence each other as they could harmonise or conflict. At the same time, they should be separated when analysing or studying the educational purposes. Therefore, Biesta (2009) suggests representing the three functions as a Venn diagram (Figure 2.8, below) to distinguish them and to emphasise the overlapping nature of their influence at the same time.

\section{Figure 2.8}

The Functions of Education Based on Biesta's (2009) Framework

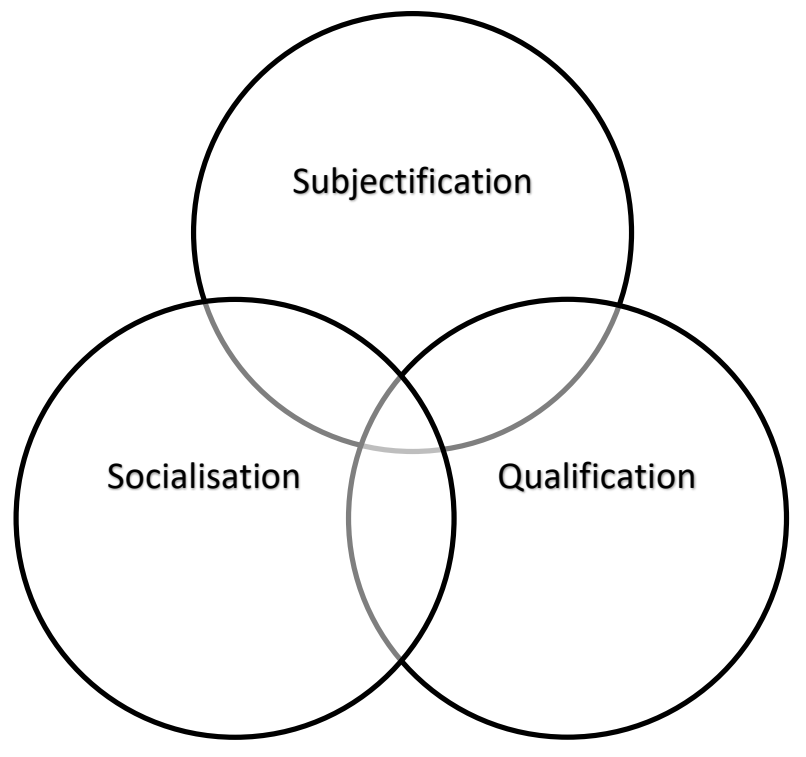

This framework was found to classify the educational objectives of classroom learning activities and provides common ground to discuss them. It emphasises the importance of educational purposes. Therefore the focus of my study is on the process, not 
the outcomes; measuring the impact of using the devices on students' learning outcomes is outside the scope of this study.

\section{Synthesising the Theoretical Framework of this Study}

There are common basic assumptions embedded in Complexity Theory and Actor-Network Theory that make them work together. Both are non-reductionist approaches that focus on the interactions and relations between heterogeneous elements, besides acknowledging the uncertain outcomes of what emerges from these interactions which make them suitable for tracing socio-materiality (Fenwick et al., 2015). Even some terms and concepts can be understood in a close manner such as element/actor, system/network.

Complexity Theory, as discussed, is more than a theory; it is a paradigm that encompasses ontological, epistemological, and methodological assumptions. While ANT is less than a theory, it is a method of how to describe and study a phenomenon. ANT moves more towards postmodernism while Complexity Theory is in the middle between realism and postmodernism. Another major difference is that although both focus on the dynamics that happen in the system or the network considering all agents or actors, ANT gives the material a more vivid notable presence. For these reasons, I used Complexity Theory as the main theoretical framework to understand the phenomenon, while I used ANT as a method that describes the phenomenon in a way that fits with Complexity Theory and focuses on digital devices as a focal point in my study. However, combining the two theories can be achieved in different ways depending on the nature and the objective of the inquiry. For example, Ali (2017) used Complexity Theory as complementary support to an ANT-based approach that aimed to describe and explain how self-sustainability of the Learning Exchange school clusters can be successful in New Zealand.

Complexity Theory is also consistent with Biesta (2009) framework of educational purposes. He urged researchers to focus on purposes instead of effectiveness and complexity instead of causality when studying educational phenomena (Biesta, 2015). His argument was based on the Complexity Theory that educational settings are "open recursive systems" that involve "reflexive intentional agents" (pp. 203-204) such as teachers and students. This implies that the deterministic causal mind-set that suits studying close systems does not provide a realistic explanation of the dynamics occurring in complex educational systems.

The assumptions of the three approaches enabled me to create a consistent theoretical framework to study the socio-materiality of the devices in classroom learning. 


\section{Summary}

This chapter presented a systematised review of recent research on using digital devices in primary classrooms. The findings showed that a considerable amount of research was conducted in relation to interventions and most of them used an experimental approach. Few studies explored students' use of devices in natural settings. There was a gap in the literature about identifying students' use in connection to the factors that shape their uses and their possible contributions to educational functions or purposes. My study synthesised a theoretical framework that adopted Complexity Theory as a paradigm that considered classrooms as complex systems. It adopted ANT to study using devices in action and adopted Biesta's (2009) framework of the functions of education to understand the contribution of using devices to the educational purposes of classroom learning activities. The next chapter will present the methodology of this study. 


\section{Chapter 3 Methodology}

This chapter describes processes and procedures followed to understand how primary students use their devices in classroom learning. It provides justifications of the methods chosen to collect and analyse data. I first outline the research design and strategy in general. Then, I describe in detail the data collection process, ethical considerations, data analysis, and trustworthiness of this study.

\section{Research Design and Strategy}

I used the model suggested by Blaikie (2009) to describe the design of my research (Table 3.1, below); however, the choices adopted in this study for each component were not limited to that model. This study is basic research, which means it is "concerned with advancing fundamental knowledge about the social world" (Blaikie, 2009, p. 49). The main two objectives of the study were to provide a thick description of the ways of use alongside an understanding of the factors that shape these uses and how the uses contribute to classroom learning objectives. The study adopted a holistic research strategy, which considers the phenomenon under study as part of a complex system; the "inquiry focuses on and captures complex interdependencies and system dynamics that cannot meaningfully be reduced to a few discrete variables and linear, cause-effect relationships" (Patton, 2015, p. 47).

The research paradigm embodies the ontological and the epistemological assumptions of the research and the methodology of collecting and interpreting the results (Bryman, 2012). The paradigm of this research, as discussed in the previous chapter, is Complexity Theory and the ontological and epistemological assumptions state that the reality and knowledge's process and components are dynamic, self-organising and emerging (Kuhn, 2007). In addition, Complexity Theory provides a conceptual framework to capture, illuminate and understand the nonlinear dynamics (Patton, 2015). Principles from ANT were engaged in this research to trace the socio-materiality of devices (Fenwick et al., 2015). Biesta's (2009) framework of educational functions, which is consistent with Complexity Theory, was employed to understand the contributions of the uses to classroom learning. 
Table 3.1

Research Design

\begin{tabular}{|c|c|c|}
\hline Component & Research choice & Explanation \\
\hline $\begin{array}{l}\text { Research topic } \\
\text { and problem }\end{array}$ & Basic research & $\begin{array}{l}\text { Advancing knowledge about } \\
\text { using devices by students for } \\
\text { classroom learning. }\end{array}$ \\
\hline $\begin{array}{l}\text { Research } \\
\text { questions and } \\
\text { purposes }\end{array}$ & $\begin{array}{l}\text { How do students use digital } \\
\text { devices for educational purposes } \\
\text { in primary classrooms? } \\
\text { Sub-questions: } \\
\text { 1. In what ways do primary } \\
\text { students use their digital } \\
\text { devices in classroom learning } \\
\text { activities? } \\
\text { 2. What factors shape the ways } \\
\text { of use in classrooms? } \\
\text { 3. How do the ways of use } \\
\text { contribute to the educational } \\
\text { purposes of classroom } \\
\text { learning activities? }\end{array}$ & $\begin{array}{l}\text { To provide a description and } \\
\text { an understanding of how } \\
\text { students use devices for } \\
\text { classroom learning, the factors } \\
\text { that shape the uses, and the } \\
\text { contributions of the uses to } \\
\text { educational purposes. }\end{array}$ \\
\hline Research strategy & Holistic perspective & $\begin{array}{l}\text { Using digital devices is one of } \\
\text { the dynamics occurring in } \\
\text { classrooms, which are complex } \\
\text { systems. }\end{array}$ \\
\hline $\begin{array}{l}\text { Research } \\
\text { paradigm } \\
\text { ontology \& } \\
\text { epistemology }\end{array}$ & $\begin{array}{l}\text { Complexity Theory: Reality, } \\
\text { knowledge, and knower are } \\
\text { dynamic, self-organising, and } \\
\text { emerging }\end{array}$ & $\begin{array}{l}\text { Blaikie (2009) } \\
\text { Patton }(2015)\end{array}$ \\
\hline $\begin{array}{l}\text { Concepts, } \\
\text { theories, and } \\
\text { models }\end{array}$ & $\begin{array}{l}\text { Complexity Theory } \\
\text { Actor-Network Theory } \\
\text { Educational functions }\end{array}$ & $\begin{array}{l}\text { (Boulton et al., 2015) } \\
\text { (Fenwick et al., 2015) } \\
\text { (Biesta, 2009) }\end{array}$ \\
\hline $\begin{array}{l}\text { Data types, forms, } \\
\text { and sources }\end{array}$ & Primary qualitative data & $\begin{array}{l}\text { Collected from natural, semi- } \\
\text { natural settings, and social } \\
\text { artefacts. }\end{array}$ \\
\hline $\begin{array}{l}\text { Selection from } \\
\text { data sources }\end{array}$ & Case study method & $\begin{array}{l}\text { Case 1: one teacher and three } \\
\text { students. } \\
\text { Case 2: one teacher and four } \\
\text { students. }\end{array}$ \\
\hline $\begin{array}{l}\text { Data collection } \\
\text { and timing }\end{array}$ & $\begin{array}{l}\text { Qualitative methods collected } \\
\text { across six months }\end{array}$ & $\begin{array}{l}\text { Observations, semi-structured } \\
\text { interviews, group interviews, } \\
\text { think-aloud interviews, } \\
\text { artefacts, video screen- } \\
\text { captures. }\end{array}$ \\
\hline $\begin{array}{l}\text { Data reduction } \\
\text { and analysis }\end{array}$ & Thematic analysis & $\begin{array}{l}\text { Abductive strategy: a } \\
\text { combination of inductive and } \\
\text { deductive strategies. }\end{array}$ \\
\hline
\end{tabular}


The data type of this research was primary, which means that, as a $\mathrm{PhD}$ researcher, I collected and analysed the data (Blaikie, 2009). The data, which were collected from different settings, were qualitative (Blaikie, 2009) to provide thorough and detailed information to describe and understand the phenomenon occurring in a complex system (Patton, 2015). I adopted the case study method (Yin, 2009) and chose to consider each classroom as a case to study the uses in relation to systems instead of case studies in relation to individuals. Collecting data from two schools took 10 weeks in each and the methods used were observations, semi-structured interviews, group interviews, informal interviews, think-aloud interviews, artefacts, and video screen-captures. Data were analysed using thematic analysis (Braun \& Clarke, 2006), and abductive strategy was employed, which is a combination of inductive and deductive strategies (Patton, 2015). The following sections describe, in detail, how each component of the research was conducted.

\section{Selection from Data Sources}

The case study method was chosen in this research to draw the boundaries around the units of analysis without isolating the phenomenon from its context, which is one of the main challenges when adopting Complexity Theory and ANT in research. A case study is "an empirical inquiry that investigates a contemporary phenomenon (the 'case') in depth and within its real-world context, especially when the boundaries between phenomenon and context may not be clearly evident" (Yin, 2009, p. 16). Blurred boundaries are associated with most of the social complex systems (Cilliers, 2001), and classrooms are no exception. The case study method gives the researcher the flexibility needed to deal with many and various variables from different data sources and "benefits from the prior development of theoretical propositions to guide data collection and analysis" (Yin, 2009, p. 17). That is consistent with the logic of inquiry in complex systems by not limiting it to a few variables interacting in a linear causal way.

Complex system sampling "involves selecting cases where complex dynamic processes can be tracked, studied, and documented over time" (Patton, 2015, p. 293). Therefore the employment of the case study method in complexity research needs the researcher to give attention to aspects related to Complex systems such as dynamics, organisation, and emergence (Anderson et al., 2005). From an ANT perspective, the researcher should study the phenomenon 'in action' (Latour, 1987), i.e., study the moments in which social relationships occur and develop. During such micro-social relations, many interactions between different interdependent agents who share history and membership are 
likely to happen, creating patterns and structures (Blaikie, 2009). With consideration to the focus of my study, which is to describe and understand the socio-materiality of digital devices in classrooms, that is, complex systems, each classroom was treated as a case.

The school year in New Zealand is divided into four terms; each term is approximately 10 weeks. Therefore, the number of cases was a crucial decision. My plan was to immerse myself and focus on one system a term; that was to understand the context deeply and to manage and organise the data collection process. Studying one classroom and spending the time allocated for data collection, which was five to six months, in one system can give a deep understanding of the dynamics of the system. Nonetheless, I wanted to study more than one complex system, not for the comparison per se, but to develop a deeper understanding of the phenomenon. Therefore, I chose to study two classrooms from two different schools for one term each. That suited my timeframe and my financial resources as a PhD student on a scholarship. Furthermore, this choice enabled me to learn and reflect on my experience in the first school before collecting data in the second one.

To achieve studying the phenomenon in action, I studied two learning activities in each class. Learning activity is defined in this research as a classroom activity designed by the teacher to achieve intended learning objectives. The activities should meet two criteria: (1) to be implemented over a sustained period of time in the term, i.e., a sequence that involved weeks, to capture as much as possible the dynamics or patterns that happen in the classroom; and (2) using digital devices is an essential component of the activity as using devcies was the focal point of this research. From my investigations in New Zealand schools, it was rare to find primary classrooms that implement many learning activities with the aforementioned criteria, since non-digital activities were still dominant at primary level. Therefore, four learning activities in two classrooms with the aforementioned criteria were suitable to achieve the objectives of the study. Additionally, I asked for a data collection extension from the University and School B as one of the learning activities was extended to term three.

Due to the objectives of this research and the focus on studying learning activity from Complexity Theory perspectives, the criteria for choosing participants were flexible. I aimed to study everyday classroom learning in natural settings without intervention. Macro and micro diversity are expected in complex systems (Boulton et al., 2015) and classrooms, indicating that any learner will enrich my research. Therefore, the main criteria for choosing 
participants were teachers and primary students who were willing to participate; the students should have diverse learning and digital skills capabilities. Choosing students depended on teachers' suggestions. Although teachers' suggestions of participants could be a possible source of bias, their recommendations were important. They knew their students better than me and, as I have mentioned before, if the bias was to happen, that would not affect the flexible criteria I had already adopted. I estimated that I would be able to observe three-four students deeply for each term. Thus, I recruited four students from each classroom, in case someone withdrew. Two teachers and seven students out of eight participated in the study until the end.

\section{Preparations for Data Collection}

Three steps were undertaken simultaneously to prepare for data collection: recruiting participants from the first school, recruiting participants from the second school, and the pilot testing of data collection instruments.

Once my research was approved by the Victoria University of Wellington Human Ethics Committee (See Appendices B \& C), I started contacting schools to test data collection methods. Since I came from a different context, an expert who knew the context well could help in choosing and finding a purposive sample (Blaikie, 2009). Thus, I had guidance from my supervisors about appropriate schools to approach for the research as they have wide knowledge, relationships, and had conducted research about digital devices in New Zealand (McRae et al., 2010; Starkey et al., 2017). This was successful in approaching the first school. In parallel, during my stay in New Zealand, I was able to develop connections and communications with teachers from different schools, which enabled me to approach the second school. In order to preserve confidentiality, the first school is referred to as School A and the second school is referred to as School B; besides, all the names used in the research are pseudonyms chosen by the participants.

\section{Recruiting Participants from School A}

I visited School A a year before starting my research with my supervisor. I noticed the active integration of digital devices in classrooms and the principal's interest in educational research. At the beginning of term one, I contacted the principal by email, who in turn invited me to discuss my research project, objectives, data collection process, and the prospective benefits of it. My research topic was relevant to the school objectives and efforts to empower their students with technological skills to become qualified and competent in the digital age. 
The principal gave me her consent to undertake my research in the school. She put me in touch with a teacher, Kathy, who was willing to participate in my research. I sent her an email to schedule a meeting and provided the information sheet for teachers.

My first meeting with Kathy took around an hour to introduce myself and explain my research project. This is was not the first time Kathy had participated in research, which made her aware of the educational research process and profits. We then discussed the learning activities to be observed and the criteria I was looking for. Kathy provided thorough information about the learning activities that require using digital devices. Two learning activities met the research criteria.

Kathy suggested several students who may be interested in participating in the study and had different backgrounds, learning strengths, and levels of digital skills. At the end of term one, I met each student individually and explained my research topic and how their participation in research would help in increasing our knowledge about using digital devices in classrooms. Then, I clarified how the data would be collected and their rights as participants. The first three students agreed to participate, the fourth student apologised, and the fifth accepted, resulting in four students out of five who were interested in participating in the research. I gave them the consent forms and the information sheets to discuss their participation with their parents. At the beginning of term two, three students provided the consent forms signed by them and their parents. I waited for the fourth student to bring back the consent forms, but he had to be absent for several weeks during the term, and there was not enough time to recruit another student. The total number of students who participated in School A was three students; one was from Year 7 and Two were from Year 8.

\section{Recruiting Participants from School B}

Getting an opportunity to undertake my research in School B was different. Through my contact with some teachers who worked in School B, I recognised that the leadership and the staff actively encourage educational research in their school. Accordingly, I sent an email to the school asking their permission to do a pilot study in one of their classrooms. As expected, two teachers volunteered to host my pilot study in their classrooms, so I chose the times that suited my schedule and thanked the other. During the testing of data collection methods, I had a chat with Tess, the teacher of the classroom, who was very interested in the topic of my research. I asked her if she would like to participate in my research in term three and she welcomed this after getting the necessary consents from the principal. 
In the middle of term two, I contacted the principal of School B, who invited me to discuss my research project. I shared all the information about my research with the principal, who thankfully gave me permission to start collecting data in term three. At that point, I contacted Tess and discussed the learning activities to be observed. Two learning activities met the criteria of my research; then we discussed recruiting student participants.

Tess prepared a list of provisional students who had diverse characteristics. I chose two students from Year 7 and two students from Year 8 because one of the learning activities would be implemented differently in each year level. I followed the same invitation procedures done with School A students; the first four students chose to participate and returned their consent forms signed by them and their parents at the beginning of term three.

In sum, this research was conducted in two primary schools, with the participation of two teachers and seven students from Years 7 and 8.

\section{Pilot Testing}

Pilot testing is a "procedure in which a researcher makes changes in an instrument based on feedback from a small number of individuals who complete and evaluate the instrument" (Creswell, 2012, p. 390). This procedure was crucial for my research. Although I had reasonable experience of doing interviews, classroom observation, and other qualitative data collection methods, doing this in a new context was a challenge. I needed to practise data collection in its natural setting and increase my confidence and awareness of the classroom environment in New Zealand before starting my research. Furthermore, pilot testing helps researchers to modify and adjust their protocols to be more efficient and effective (Creswell, 2012). For example, I had to check that the questions used in the interviews were understandable for the participants, the time estimated for the interviews was accurate, the observation protocol was manageable, and the methods used would capture what was intended to be captured.

I first tested the teacher's interview with a $\mathrm{PhD}$ colleague who used to work as a primary teacher in New Zealand. I used her feedback to modify the questions and adjust the length of the interview. I also tried data collection methods with students (focus group interview, think-aloud interviews) with friends' children who were in Years 7 and 8 in New Zealand schools in their homes. I was then confident enough to visit School B to test data collection instruments in an authentic classroom environment. No data were collected or recorded; only my notes, my reflection, and the feedback from the teacher and children were 
recorded. After that, I discussed with my supervisors the changes and the modifications to be made so that the data collection protocols were revised and ready to be used for the research.

It is worth mentioning here, that although I used the same data collection methods during the research period, my experience in observing classroom dynamics, asking questions, and recording notes were enhanced over time. This process manifested the epistemological position taken in this research that the knower, the known, and the relationship between them are dynamic, self-organising, and emerging (Kuhn, 2007). It will be contrary to the logic of the research paradigm, and reality, claiming that the first interview was conducted the same as the last one; the same applies to the rest of the methods. What I can confirm is that I was committed to maintaining the trustworthiness of the inquiry during the whole period of the data collection process.

\section{Data Sources and Data Collection Methods}

Data collection started at the end of April 2018 and ended in the middle of October 2018. I spent term two (10 weeks) in School A observing two learning activities in one classroom. I then spent term three (10 weeks) in School B observing another two learning activities in one classroom. Collecting data in complex systems should be responsive, adaptive, and flexible (Patton, 2015), especially as I aimed to capture the dynamics as they happened, without intervention. Observations happened weekly except for one week in each school due to changes in teaching plans. Data were collected from three sources: natural settings, seminatural settings, and social artefacts.

\section{Collecting Data from Natural Settings}

Collecting data from natural settings means collecting data directly from the context while the phenomenon is in action (Blaikie, 2009). This type of data was essential to study the phenomenon in action (Latour, 1987) and to capture factors influencing using devices that might not be reported by participants. Three methods were used: observation, recording participants' screens, and think-aloud interviews.

Observation. This method was used to collect primary data by observing the phenomenon systematically (Cohen et al., 2017). Observation served the purpose of my study because it provided a thick description of the contexts, actors, interactions, and mundane events which are usually overlooked (Patton, 2015). My role as a researcher was a 'nonparticipant observer' (Creswell, 2012). That was to observe using devices as it occurred in the normal and usual conditions, which means that data was collected without research 
intervention. During learning activities, I sat behind the participant in a position that enabled me to observe their screens and their interactions with others.

What and how to observe can be challenging in a complex system, therefore there must be a focus (Patton, 2015). Research questions and 'following the actors' strategy (Latour, 1987) guided what to observe and how. Once the learning activity started, I followed one of the participants at a time; I documented how the student used their devices and what interactions accompanied the uses. I followed the learning activities from the beginning of the term until it ended. I aimed to attend all the sessions of these learning activities attended by the participants, thus, I conducted 14 observations in the first classroom, and 13 observations in the second classroom ranging from 30 minutes to an hour and a half each.

Think-aloud interviews. This method is to invite the participants to talk about what they are thinking while doing an activity and recording their response (Young, 2005). I was concerned about using this method with the children because when I tried this method with adults it was challenging to understand what 'talking about your thinking' means. For this reason, I used a strategy implemented in a previous study (Starkey, 2010), which was to prepare some short, direct questions as 'prompts'. That made it easy for the student to manifest their thinking while they were using their devices. These questions were, for example: what are you doing? How are you doing it? Why are you doing that? Is it easy/hard? Why? Is it new? And what have you learnt? (See Appendix E).

This method aligned with the objective of the study to understand the phenomenon in action. It exhibited students' hidden accounts (Young, 2005), which increased my understanding of the observed events. This method was useful because some incidents were better investigated directly rather than waiting until the participants reported about them in the interviews. A point to be made is that the nature of the classrooms that I observed allowed this method to be successful. The classroom environment enabled the students to move, talk, and interact with each other, which made this kind of communication between the researcher and the students possible and usual.

Using this method required taking different factors into account, such as timing, length, and the characteristics of the student. Some of the participants engaged quickly and gave detailed explanations and information. Others spoke briefly with short answers and were not ready to talk sometimes. Therefore, the length of these interviews varied significantly. In general, the participants were cooperative and responded most of the time to the think-aloud 
interviews. I recorded these interviews using an audio recorder and sometimes used Screencastify recorder, as explained next. Then they were transcribed; the audio files and transcription files were uploaded to the qualitative analysis software.

Video screen captures. In some events when participants were using digital devices, I asked them to video record their screens and then share these recordings with me to analyse. For this purpose we used 'Screencastify', which is open-source software that can be installed on web browsers and records students' screens and their conversations (Israel et al., 2017). This technique provided valuable data because it recorded how students use their devices to interact with different actors, such as teachers, peers, and technology. It showed how some digital artefacts and documents were produced and shared. It was used sometimes to record think-aloud interviews simultaneously. Five participants out of seven used Screencastify and shared their recordings with me; two students were not familiar with it and did not feel comfortable using it. Another issue was that Screencastify, in the free mode, only records 10 minutes, so the students sometimes had to record their activity intermittently. This method enhanced the reliability and accuracy of the observations. It shed more light on the factors that influence using the devices.

\section{Collecting Data from Semi-Natural Settings}

Collecting data from semi-natural settings means that "individuals are asked to report on their activities that occur in natural settings" (Blaikie, 2009, p. 163). That required collecting demographic data about the participants and interviewing participants to give an account of their opinions, understandings, activities, and behaviours. Three types of interviews were used in this study; two were with the teachers and included semi-structured interviews and informal conversational interviews with the teachers. Group interviews were conducted with the students.

Semi-structured interviews with teachers. Asking participants open-ended questions is a useful method to explore their understanding of phenomena without being limited to the researcher's theoretical assumptions (Bryman, 2012). This method allowed participants to clarify the factors that influence the use of digital devices and how using devices might contribute to their educational purposes. It added to my understanding of the phenomenon by linking my observations with how teachers reported and evaluated the events. 
To preserve consistency across the participants and to capture the uniqueness of each context, I adopted Patton's (2015) suggestion to use a strategy that incorporates “specifying certain key questions exactly as they must be asked while leaving other items as topics to be explored at the interviewer's discretion" (p. 441). This strategy aligned with Complexity Theory's epistemological stance about the dynamic nature of knowledge, while at the same time preserving the focus of the research.

I conducted two interviews with each teacher. The first one was at the beginning of the term, and the other one was after the term had ended. In the first interview, the questions were about demographic information, the teacher's experience, perceptions about learning in general, using digital devices in classrooms, factors that impact using them, how they plan to use them for the learning activities, and the objectives of the learning activities. At the end of the term, the questions focused on how teachers had organised and implemented the learning activities, what factors influenced using devices by the students, how they evaluated the learning activities and students' learning in light of learning objectives, the pros and cons of using digital devices, expected and unexpected outcomes, and changes they would make in the future (See Appendix D).

The questions, as mentioned, were modified to fit the two contexts. For example, there were some questions about specific events that happened during the term, questions about some decisions taken by the teacher, and about their students as a learner. Each interview was audio-recorded then transcribed and lasted for about 60 minutes.

Informal, conversational interviews with teachers. This method means that the researcher asks "questions [that] emerge from the immediate context" (Patton, 2015, p. 438) to clarify questionable situations. The main advantages of this method are "flexibility, spontaneity, and responsiveness to individual differences and situational changes" (Patton, 2015 , p. 437). On the other hand, it is less systematic and can be problematic while analysing data. The dynamic nature of the classrooms and the unexpected events required using this type of short interview despite its limitations. During the term, whenever was suitable I asked the teachers about certain events that needed clarification. These questions were sometimes sent by email but most of the time were asked at the end of the day. The teachers' answers were recorded on my notebook on separate pages or next to the questionable situations to reduce the expected difficulty during analysis. 
Group interviews with students. Group interview "is the process of collecting data through interviews with a group of people" (Creswell, 2012, p. 218). Literature suggests that group interviews encourage children to express themselves and feel more comfortable, especially if the questions are direct and clear (Cohen et al., 2013). Other reasons for choosing this method were the advantages mentioned by Patton (2015), such as effectiveness, especially as I did not want to take up students' valuable learning time in school or distract from classroom routines. In addition, the method can manifest the different perspectives, the evaded issues, and the interactions among the participants. Finally, Patton (2015) suggested that participants are likely to enjoy this type of interview.

The same questioning strategy used with teachers' interviews was used; fixed questions to both groups and other questions that emerged from their context. The general questions included: what did they learn from the learning activities? What obstacles have they faced? How did they overcome them? How did digital devices help or not in doing their tasks? And what other factors helped or can help them to learn better? (See Appendix E). I conducted one group interview in each school. At School A, all three students participated in the interview. At School B, three out of four participants joined the interview because the fourth student was absent. Both interviews were audio-recorded then transcribed and lasted for about 45 minutes.

Managing and facilitating the focus group interview, which are major limitations of this method, were easier than expected. The pilot testing was beneficial for me to practise adjusting the time and distributing the questions. Also, my experience as a primary teacher was necessary for building my confidence in front of children, empathy, and consideration while dealing with them. Another significant factor was that both groups of students were used to engaging in group discussions in their classrooms and to showing respect to others' turns and opinions. As confidentiality is not guaranteed in group interviews (Patton, 2015), I explained the meaning of confidentiality to the participants before starting.

\section{Collecting Social Artefacts and Documents}

Artefacts usually refer to three-dimensional objects, while documents refer to "a wide range of written, visual, digital, and physical material relevant to the study (including visual images)" (Merriam \& Tisdell, 2015, p. 162). However, in my study, these terms are used interchangeably, as both are "traces of social activities left behind by participants" (Blaikie, 2009, p. 170). A considerable number of artefacts were collected. Artefacts collected from 
students included digital and non-digital products (Krajcik \& Shin, 2014); I took photos of the non-digital products. The digital artefacts included digital assignments, designs, and documents, and the non-digital included their notebooks, display boards, and printed work. Digital artefacts were subject to change and updating by participants during the term; therefore, I used all versions for analysis when needed. Literature suggests that exploring the process of producing artefacts by students can illustrate how learning emerges individually and collectively, through various negotiations, feedbacks, and relationships besides the fact that these artefacts are evidence of learning outcomes (Krajcik \& Shin, 2014). Artefacts were also collected from teachers, schools, and the New Zealand Ministry of Education website and included digital and non-digital documents, as they offer valuable sources of qualitative data (Patton, 2002). They included lessons and slides created by teachers, classroom timetables and posters, school curriculums, schools' documents on the websites, and the New Zealand Curriculum. All the artefacts were organised and analysed using MAXQDA.

\section{Summary of the Data sources and Collection Methods}

The amount of data from different resources achieved data saturation (Creswell, 2012). Table 3.2, below, summarises all the data collected for this study. 
Table 3.2

Summary of Collected Data

\begin{tabular}{|c|c|c|}
\hline Sources & Methods & Collected Data \\
\hline \multirow[t]{3}{*}{$\begin{array}{l}\text { Natural } \\
\text { settings }\end{array}$} & Classroom observations & $\begin{array}{l}14+13=27 \\
\text { c. } 30-90 \text { minutes each }\end{array}$ \\
\hline & $\begin{array}{l}\text { Screen captures of } \\
\text { students' screens }\end{array}$ & $\begin{array}{l}9+4=13 \\
\text { c. } 9-30 \text { minutes each }\end{array}$ \\
\hline & $\begin{array}{l}\text { Think-aloud interviews } \\
\text { with students }\end{array}$ & $\begin{array}{l}14+12=26 \\
\text { c. } 3-10 \text { minutes each }\end{array}$ \\
\hline \multirow{3}{*}{$\begin{array}{l}\text { Semi- } \\
\text { natural } \\
\text { settings }\end{array}$} & $\begin{array}{l}\text { Semi-structured } \\
\text { interviews with teachers }\end{array}$ & $\begin{array}{l}2+2=4 \\
60 \text { minutes each }\end{array}$ \\
\hline & $\begin{array}{l}\text { Group interviews with } \\
\text { students }\end{array}$ & $\begin{array}{l}1+1=2 \\
45 \text { minutes each }\end{array}$ \\
\hline & $\begin{array}{l}\text { Informal interviews } \\
\text { with teachers }\end{array}$ & $\begin{array}{l}\text { Occasional questions, at the end of learning } \\
\text { activities or via email }\end{array}$ \\
\hline \multirow[t]{3}{*}{$\begin{array}{l}\text { Social } \\
\text { artefacts }\end{array}$} & Students' artefacts & $\begin{array}{l}\text { Products (digital and printed) that required using } \\
\text { digital devices such as texts, graphics, spreadsheets }\end{array}$ \\
\hline & Teachers' artefacts & $\begin{array}{l}\text { Instructions on classroom walls } \\
\text { Documents shared with students such as slides, } \\
\text { assignments, instructions, timetables }\end{array}$ \\
\hline & $\begin{array}{l}\text { Other } \\
\text { Documents }\end{array}$ & $\begin{array}{l}\text { School curriculum } \\
\text { School newsletters } \\
\text { Information on School websites } \\
\text { New Zealand Curriculum }\end{array}$ \\
\hline
\end{tabular}

\section{Ethical Considerations}

Ethical considerations are fundamental and crucial in any research and require special attention when researching with children (Cohen et al., 2013). I followed Human Ethics Committee policies and guidelines from Victoria University of Wellington. Participation in this study was voluntary at all stages of the research. Informed consent was obtained from school principals, teachers, and parents/guardians alongside with children's assent. I have ensured confidentiality, so all the names used in this study are pseudonyms; each child chose their own pseudonym, and all data were kept in a University-owned, password-protected cloud drive.

My experience as a primary teacher helped me to ensure the four rights embedded in the United Nations Convention on the Rights of the Child (UNCRC) (i.e. welfare, protection, provision, and choice and participation) (Powell et al., 2012) as follows. First, the finding of the study will add to children's welfare by increasing our knowledge about using digital 
devices for learning. Second, I chose data collection methods that minimise harm to children; for example, I used think-aloud interviews to collect data without interrupting students' learning. Additionally, I chose group interviews to reduce stress and anxiety and save students time in school (Cohen et al., 2017). Third, in the information sheet, I explained to the children the importance of the research and how their participation will benefit human knowledge and help teachers and educators in their work. Finally, children and their parents were given a choice to participate in the study by consent and assent. I took into consideration children who did not participate in the study; I listened to them, gave attention, and respected any child who shared his/her notes with me; at the same time, data were only collected from those who chose to participate. In addition, in consideration for the busy nature of the teaching profession, I did my best not to increase the workload of the participating teachers as far as possible.

\section{Data Analysis}

"Qualitative analysis transforms data into findings" (Patton, 2015, p. 521). The fundamental inquiry of this study was how primary students use their digital devices for classroom learning. To find answers for this overarching question I outlined three focuses: the uses, the interacting factors, and the contribution. Each of these focuses evolved into the three subquestions (Braun \& Clarke, 2006) and became more focused during the analysis process. That reflects the ontological and epistemological assumptions adopted in my research, which see reality as dynamic, self-organising, and emergent. Therefore, the analytical framework of this study was designed based on these three focuses and was flexible to align with the paradigm assumptions.

The analysis approach of this study was the thematic analysis suggested by Braun and Clarke (2006); it is "a method for identifying, analysing, and reporting patterns (themes) within data. It mainly organizes and describes your data sets in (rich) details” (p. 79). The application of the six phases of thematic analysis is summarised in Table 3.3, below. The thematic analysis reflects the dynamic, emergent nature of knowledge. Writing the cases started at phase one, as writing is a vital analytical activity that should accompany the whole analysis process. The phases were intertwined, as the process was nonlinear and recursive. I considered each classroom as a case that represents a complex system. I first analysed the data collected from the first case. Then the analysis and the report of the findings were reviewed by my supervisors. Their feedback led to the development of an analytical 
approach, which was applied to the second case study. The analytical approach was revised again and modified and then applied to both cases for consistency.

The nature of the findings of each question required a different coding strategy. A purely inductive or deductive strategy did not fit with the purposes of the inquiry. Therefore, I used an abductive strategy, which is a combination of inductive and deductive strategies. It means "working from consequence back to cause or antecedent. The observer records the occurrence of a particular event, and then works back in time in an effort to reconstruct the events (causes) that produced the event (consequence) in question" (Denzin, 1978, pp. 109110). To identify the uses of digital devices, that is, the first research question, I mainly used the inductive strategy, which means "coding the data without trying to fit it into a pre-existing coding frame, or the researcher's analytical preconceptions" (Braun \& Clarke, 2006, p. 83). That resulted in seven uses of digital devices, which became basic themes when coding the other two questions.

When coding the second question, I read the data many times, as a detective, and created a matrix (Patton, 2015) to find evidence of the factors that most likely shaped the uses. The factors were classified in relation to different elements interacting in the classroom. The classification took the contextual factors into account.

For the third question, I initially coded the contribution of the uses based on objectives chosen by the teachers; the objectives in the first case were based on the five key competencies of the New Zealand Curriculum (Ministry of Education, 2007). That made it a more deductive-oriented analysis as data were coded based on pre-existing coding frames (Braun \& Clarke, 2006). For the second case, I had to inductively extract the objectives from the data before coding the contribution of the uses. After that, the objectives were deductively classified based on Biesta (2009) framework.

When identifying the themes in the final phases, literature was reviewed. This step is recommended for abductive analysis as that could assist in ordering the data and in finding "concepts that can capture fundamental differences between social actors/views or actions" (Ong, 2010, p. 5). A summary of the analysis approach is presented in Table 3.3, below. 
Table 3.3

Analysis Approach

\begin{tabular}{|c|c|c|c|}
\hline $\begin{array}{l}\text { Thematic } \\
\text { analysis Phases }\end{array}$ & $\begin{array}{l}\text { RQ1: focuses on } \\
\text { identifying the uses } \\
\text { of digital devices }\end{array}$ & $\begin{array}{l}\text { RQ2: focuses on } \\
\text { factors shaping the } \\
\text { uses }\end{array}$ & $\begin{array}{l}\text { RQ3: focuses on uses' } \\
\text { contributions to } \\
\text { educational functions }\end{array}$ \\
\hline $\begin{array}{l}\text { 1. Familiarising } \\
\text { with the data }\end{array}$ & \multicolumn{3}{|c|}{$\begin{array}{l}\text { Audio and video files were transcribed. Files were organised into a } \\
\text { database for each case on MAXQDA } 2018 \text { (updated to 2020) software. }\end{array}$} \\
\hline $\begin{array}{l}\text { 2. Generating } \\
\text { initial codes } \\
\text { 3. Searching for } \\
\text { themes } \\
\text { 4. Reviewing } \\
\text { the themes }\end{array}$ & $\begin{array}{l}* \text { Inductive strategy } \\
* \text { Initial codes } \\
\text { extracted from data } \\
\text { then collated into } \\
\text { themes } \\
\text { *Seven themes } \\
\text { represented seven } \\
\text { uses }\end{array}$ & $\begin{array}{l}\text { *Investigated data to } \\
\text { code factors related } \\
\text { to each use } \\
\text { *Coded factors were } \\
\text { classified in relation } \\
\text { to five themes } \\
\text { (elements) } \\
\text { considering } \\
\text { contextual aspects }\end{array}$ & $\begin{array}{l}\text { *Deductive and } \\
\text { inductive strategy } \\
\text { *Coding contributions } \\
\text { based on learning } \\
\text { objectives chosen by } \\
\text { teachers } \\
\text { *Classifying learning } \\
\text { objectives and uses' } \\
\text { contribution based on } \\
\text { Biesta's (2009) } \\
\text { framework }\end{array}$ \\
\hline $\begin{array}{l}\text { 6. Producing the } \\
\text { report }\end{array}$ & $\begin{array}{l}\text { Organised the finding } \\
\text { samples from the cod } \\
\text { by researcher and sup }\end{array}$ & rvisors. & $\begin{array}{l}\text { earch questions, extracted } \\
\text { ort, reviewed several times }\end{array}$ \\
\hline
\end{tabular}

\section{Trustworthiness}

Scholars argue that validity and reliability criteria used to evaluate quantitative research cannot be applied to qualitative research (Cohen et al., 2017). Lincoln and Guba (1985) suggest four criteria to evaluate qualitative research: credibility, transferability, dependability, and confirmability.

Credibility replaces the 'internal validity' concept used to validate quantitative research (Cohen et al., 2017), which refers to the truthfulness of the findings. For this study, I used several techniques suggested by Lincoln and Guba (1985) for establishing credibility. First, 'a prolonged engagement' provides the scope in which the researcher can identify the factors that influence and shape the phenomenon. I was able to achieve this by spending 10 weeks collecting data from each classroom. That enabled me to better understand the context and the culture of each class alongside developing relationships with the school's communities. Second, 'a persistent observation' provides the depth essential to understand 
the observed uses and factors and how they interact with each other. Therefore, I chose to intensively focus on three to four participants in each classroom to be able to observe them persistently instead of superficially observing many participants. Third, 'triangulation' is understood in this study as a technique that offers holistic and rich data instead of a method for inconsistency settlement (Patton, 2015). Therefore, I used seven data collection methods from three different settings to acquire rich information about the phenomenon. Fourth, 'member checks' are considered by Lincoln and Guba (1985) to be the most crucial method for qualitative research credibility. That was achieved by sending the findings chapters that included the data, my analysis and interpretations, and conclusions to the participant teachers. Both teachers confirmed the accuracy of the findings and that they reflected their experience. The teacher who participated in the first case clarified some points by giving extra information, corrected some information that was reported in the interviews, and corrected one error in the transcription of her interview.

Transferability is a method in which the researcher provides a thick description of the phenomenon that allows the reader to evaluate to what degree the findings can be transferred to other contexts (Lincoln \& Guba, 1985). This method is the qualitative version of the 'external validity' criterion used in quantitative research (Cohen et al., 2017). Providing a thick description of the contexts was not just a method for trustworthiness but also a main objective of the study. The findings chapters provide rich details of how students used the devices and the contexts that surrounded these uses. As the researcher came from a different educational context, the small details of classroom dynamics were as important for me to describe as the major details, because that will help educators from other contexts to reflect on the similarities, differences, and possibilities.

Dependability is a method used to evaluate qualitative research, which replaces the concept of reliability. A reason for not using reliability for qualitative research is that qualitative inquiry is based on uniqueness, not replicability (Cohen et al., 2017). Dependability can be achieved by external audit, which refers to examining the process of producing qualitative research (Lincoln \& Guba, 1985). It was achieved by the consistent feedback received from my supervisors and the examination process of the thesis.

Confirmability can be achieved when all the three aforementioned criteria are met (Lincoln \& Guba, 1985), which includes member checks, external audit, and triangulation. In addition, rationales underpin the theoretical framework and methodology, and analysis should 
be clarified for the readers to show how findings and conclusion have been derived (Nowell et al., 2017). This aspect required using sound and efficient tools to manage the data, which was achieved by using MAXQDA 2020 for qualitative data analysis. The study demonstrates the reasons behind the theoretical and methodological decisions which led to the final conclusions; that is, to enable the reader to evaluate the confirmability of the study.

\section{Summary}

This chapter described in detail the research design and strategy. Two teachers and seven students from two classrooms in two schools participated in this study. They were observed while undertaking four learning activities that required using digital devices. The case study method was used; the sources of the data included collecting data from natural and seminatural settings alongside social actors' artefacts. The methods used were observations, semistructured interviews, group interviews, informal interviews, think-aloud interviews, artefacts, and video screen-captures. Data were analysed using thematic analysis and abductive strategy. The ethical considerations and the trustworthiness of the study were addressed. The next two chapters report the findings of the cases, followed by findings' synthesis, and then discussion. 


\section{Chapter 4 Case Study One}

This chapter reports the findings of the first case study. First, I provide background information about the context, the participants, and the observed learning activities. Then I report the findings related to the ways of use and the factors that shaped them. Finally, I report how the ways of use contributed to educational functions based on the objectives of the learning activities.

\section{The Context: The School and the Classroom}

School A was a full primary school from Years 1 to 8 in a low-income urban area in New Zealand. It had nearly 340 students drawn from a culturally diverse community, with approximately 27\% Māori students and 66\% Pacific heritage students studying in an Englishmedium education system. The school's main priorities, as stated in school documents, were to help students to be proud of their cultures and communities and to become confident and skilled for the future as life-long learners. The school curriculum reflected these priorities through emphasising culturally responsive practices and engaging students' cultures in many learning activities. In addition, the school was adopting various learning approaches such as STEAM workshops, Play-Based Learning, and Inquiry-Based Learning, as key pedagogical approaches in the curriculum of the school. The students were required sign an agreement named 'Kawa of care' to organise using digital devices in the school. Teachers explained this agreement to students, then the students had to demonstrate their understanding of it through an interview. Finally, they and their parents had to sign it before bringing their devices to school.

The classrooms were designed as Innovative Learning Environments (ILE). The classroom, in which the learning activities were observed, had a communal space, three breakout spaces, a book corner, a projector, three iMacs, two whiteboards, storage places for students' digital devices and stationery, a small kitchen area, and tables and chairs of different sizes and shapes. At this space, there were three classes of Year 7 and 8 students with their three teachers learning together. They usually gathered in the communal space to take directions from one of the teachers; then the students were distributed among different spaces with a teacher to start their learning activities. The teachers worked together, as the school adopted a collaborative teaching model to facilitate teaching in the ILE. The students used mainly Chromebooks to do their learning activities. There were also a few iMac desktop computers that were used occasionally, for example, when someone's device stopped 
working. In that context, a teacher and three students participated in this study. The following section introduces them.

\section{The Participants: A Teacher and Three Students}

When I visited School A, Kathy, a teacher of Year 7 and 8 students, volunteered to participate. I asked her to suggest four students who had different learning capabilities to participate in the study. I invited five students; four accepted. At the beginning of the third week, the fourth student stopped coming to school so I could not collect data related to him. The other three students, Mark, Peach, and Sam, participated in the study until the end of the term.

\section{Kathy}

Kathy had graduated from a teachers' college in New Zealand more than 30 years ago. She started her career as an itinerant teacher of Te Reo Māori, then chose to join School A because of how the staff treated her when she worked in that school. She said that she had a strong sense of belonging to the school: "I got to realise that some schools were places where you feel like you belonged, and you were valued, and you were supported, and it was quite interesting ... I really enjoyed the staff'. Kathy mentioned her long experience with the children and her interactions with the community where the school was situated: "I'm part of the community, in a way, I don't live in the community, but I have lots and lots of links". Kathy was an active teacher who participated in different educational and professional development initiatives. During my visits to the school, I saw her contributing in several areas such as selecting inspirational quotes about education to place on the staffroom wall, playing the guitar with the students at school Hui, and participating in after-school activities.

\section{Sam}

Sam was a student in Year 7. He was a quiet boy who liked music and always smiled. Sam believed that learning is about problem-solving. He trusted his teacher and sought her attention while working. He was easily influenced by the opinions of his older peers and repeated their sentences. Kathy suggested him as he had moderate digital skills compared to other students. When using his device, he needed time. He sometimes asked his friends questions or shared his work with them to get feedback, but most of the time, he liked to sit in a corner alone or under the table to work on his device to focus on his learning. 


\section{Peach}

Peach was a Year 8 student. She was talented in music, arts, and sports. Peach was usually the leader when she worked in a group. When she was working alone, her friends approached her to ask questions and to get her opinion about their work. She believed that learning was "discovering new things". She was digitally competent. She was able to use different applications easily. When she worked on her device, she was focused and took her work seriously.

\section{Mark}

Mark was a Year 8 student. He liked basketball and digital technologies. Kathy and other teachers praised his maths and writing skills. He had advanced digital skills compared to the other students, which allowed him to work on the computer of the school library. He was the "problem-solver" for his peers when they faced a problem on their computers, which made him very popular among them. He preferred working with his peers and was always helpful and cooperative, which was obvious during the 10 weeks of the observations. Mark believed that learning was "like filling up your brain and like just knowing things".

\section{The Observed Learning Activities}

Kathy suggested two learning activities that required the students to use digital devices. The first one was 'STEAM workshops'. The second one was 'Literacy'. The next section provides a general description of the activities.

\section{STEAM Workshops}

'Who can I be in the future' was the title of the learning activity that was prepared by Kathy and other teachers for Year 7 and Year 8 students as their three classrooms studied together. The workshops were guided exploration workshops based on a STEAM framework. The purpose of the workshop was outlined in a document shared by the teacher with students:

Purpose: We will continue to develop our identity with a future-focus. We will develop our voice and agency through confident, clear communication, taking risks, trying new things and sharing our learning in a variety of ways. We will be exploring new ideas and concepts with a STEAM Science, Technology, Engineering, the Arts, and Maths and future focus.

Kathy explained, "the purpose was to give them a taste of some different ideas and skills and ways of working together". She added that these workshops not only aimed to introduce areas of work in the future but also the options within these areas; she gave the following example: 
"In the future, I could be a musician ... but within that musician, I could be a creator of music. I could be a player of music". Another objective was to prepare the students for the next learning activity in term four that required making their "own inquiry about their passion".

The workshops covered different topics that integrated the different subject areas of the STEAM framework. For example, there were: Sign Language Workshop, Making a Sweet-Smelling Natural Hand Sanitiser, 3D Images, Music Technology, and Kōwhaiwhai Printmaking. These workshops were run by teachers and volunteers from the community, such as a science teacher, a rugby player, and a social worker, Kathy said:

For me, when I ran workshops, there was a lot about the Arts, you know, the music and visual language and drama because it's my strength. And so maybe for Sara [another teacher], hers were a little bit more about science or technology because there's her strength, and I know we did have a lot of people come in from the community who did all sorts of different things.

Each week Kathy shared a document on Google drive with her students that contained a table describing nine to 13 workshops the students could choose from. The table, as in Figure 4.1, below, provided information about each workshop, including the time and date, the title and the description, the resources needed, and the names of the students who will participate in the workshop. Out of these weekly workshops, there were usually one or two workshops that required using Chromebooks.

\section{Figure 4.1}

Sample of the Weekly Table of STEAM Workshops

TERM 2 WEEK 5: 28th- 1st June

\begin{tabular}{|c|c|c|c|}
\hline DATE/ TIME & WORKSHOP TITLE \& DESCRIPTION & STUDENTS & $\begin{array}{l}\text { RESOURCES: Please } \\
\text { bring these resources to the } \\
\text { workshop if you have them }\end{array}$ \\
\hline $\begin{array}{l}\text { Tuesday } \\
11.30-12.30 \\
\text { AND } \\
\text { Wednesday } \\
11.30-12.30\end{array}$ & $\begin{array}{l}\text { 3D Modelling } \\
\text { Being 3D modellers to create some emoticons for } \\
\text { Sia to help her teach Samoan Language. }\end{array}$ & $\begin{array}{l}24 \text { students (who haven't already done } \\
\text { this workshop) }\end{array}$ & chromebooks \\
\hline
\end{tabular}

In the first two weeks of the term, Kathy discussed with her students the upcoming workshops and the concept of STEAM. During this time, I was able to familiarise myself with the context; Kathy introduced me to the students and explained my role as a researcher 
in the classroom. I talked with the participants to let them get to know me and to answer their questions. I usually sat behind the students to make them feel comfortable.

The workshops started in week three and ended in week ten. In week eight, the students did not do this activity as they had to participate in another activity outside the school. There were three days in the week assigned to the workshops alongside a Play-Based Learning activity and critical literacy in the library. On Mondays, each student had to choose three workshops to participate in during the week, except if the workshop ran over two days. If the workshop ran over two days, the student needed to attend both sessions.

Based on my observations, the workshops could be divided into three phases: induction, implementation, and evaluation. In the induction phase, the topic and the objectives of the workshop were discussed with the students. The basic concepts related to the topic, the success criteria, and what makes the project successful were explained. Kathy explained one of the main objectives of this phase as "some success criteria are coconstructed with the students, i.e., what do we already know that will make us successful at solving this problem/answering this question? Then more success criteria are added as we complete the learning task". Additionally, an explanation of 'how to do' the project was provided with online resources and links shared with the students to help them while working on their tasks. The basic rule in this phase was announced by Kathy: "Put your devices away until you know exactly what you have to do". Thus, the use of the devices in this phase was limited and supervised by the teacher.

The implementation phase was assigned to students to carry out their projects. During this phase, the participant students sometimes interacted with their peers while working on the devices. That was to exchange information, skills, and experiences about 'how to do' things or to give feedback and opinions about each other's works. At other times, they preferred working alone. Kathy said that teachers might do some noticing, that is, observing students during learning, to evaluate students' learning while students were working. The last phase was the evaluation phase; the students were given the opportunity to present and share their work with their teacher and peers. Kathy explained, "We always have a connect at the end, so we all share our ideas, and then we analyse that".

Kathy used another strategy to evaluate students' learning alongside teachers' noticing: 
That would be the kids talking and sharing their success in terms of success criteria. They say we were good at choosing the best website, that we could understand, to be able to find out the information, and we were good at persevering when we got stuck with how to make the shapes fit together. So that would very much come from the children. We might do some noticing as well. And we would reflect on that lesson, but we would reflect on the lesson based on kids' success criteria.

Another strategy for evaluation was the 'learning stories'. Learning stories were narratives created by the students to record their thoughts, memories, photos, and reflections about their learning experiences. They were used for all learning activities in the classroom including STEAM workshops. Learning stories were saved on students' Google drives and posted on their blogs where teachers, peers, and family members could access and comment on them.

Before each workshop started, Kathy and the other teachers gathered the students from the three classrooms to give instructions. The students were then distributed to their positions with other teachers, whether the workshops, the Play-Based Learning activity, or the library for a critical literacy activity.

For the purpose of this study, I only observed Mark, Peach, and Sam in workshops that required using Chromebook. These workshops were Music Technology, Google Logo workshop, and two 3D Modelling workshop. Each of the four workshops ran for two sessions.

Music Technology workshop: In this workshop, I observed Sam who was expected to choose a video clip from a list and compose a piece of music that triggered a certain feeling such as sadness, happiness, or thrill. The students were introduced to an application called 'SoundTrap' (Spotify USA Inc, 2018) to be used for their projects.

Google Logo workshop: In this workshop, I observed Peach during designing her Google logo using 'Scratch' software. The purpose of this workshop was that each student created a Google sign that told a story about themselves.

3D Modelling workshop: I observed this workshop twice; once to observe Sam and another time to observe Mark. The participants were required to design 3D images of faces with different emotional expressions such as the angry face, happy face, and sad face to help a teacher aide in teaching Samoan Language. The software used was 'SculptGL' (Ginier, 
2018), which the students had used before in another learning activity. Mark was absent in the first session because he was sick but attended the second session the following day.

There were two workshops I did not observe; one was held in another school about robotic programming and was attended by Mark. The other one was about graphic design and attended by Peach. I have included what Mark and Peach wrote about these two workshops in their learning stories as part of the collected data. There was also one workshop, attended by Mark, for which I just observed the beginning. That was because using the devices was optional, and Mark decided not to use his. As a researcher, I did not intervene with students' choices, and I was lucky that their choices did not overlap. That helped me to focus on each student individually when they interacted with their devices. I did not observe some workshops that required using digital devices because none of the three participants were enrolled in them.

\section{The Literacy Learning Activity}

Students used their devices for 'critical literacy' and 'cool quick writing', which both ran through the whole school year. Critical literacy was a group work learning activity of threefour students per group. Every week, the teacher used Google drive to share a document (Figure 4.2, below) including the title of the activity, the date, and the instructions to do the task. It also contained a link to a specific piece that could be a journal story, an article, or a clip. These pieces were selected from educational websites or books. Kathy pointed out the objective of this activity:

We really wanted some evaluative thinking, and so it was around what I think? what I know? what I believe? ... it's also looking at seeing if they [the students] can identify some bias in the text ... [giving examples] "I disagree with that because" and "I think the writer's only showing one side of that", "They've only presented one part of the argument", and so [on]. 


\section{Figure 4.2}

A Sample Critical Literacy Assignment

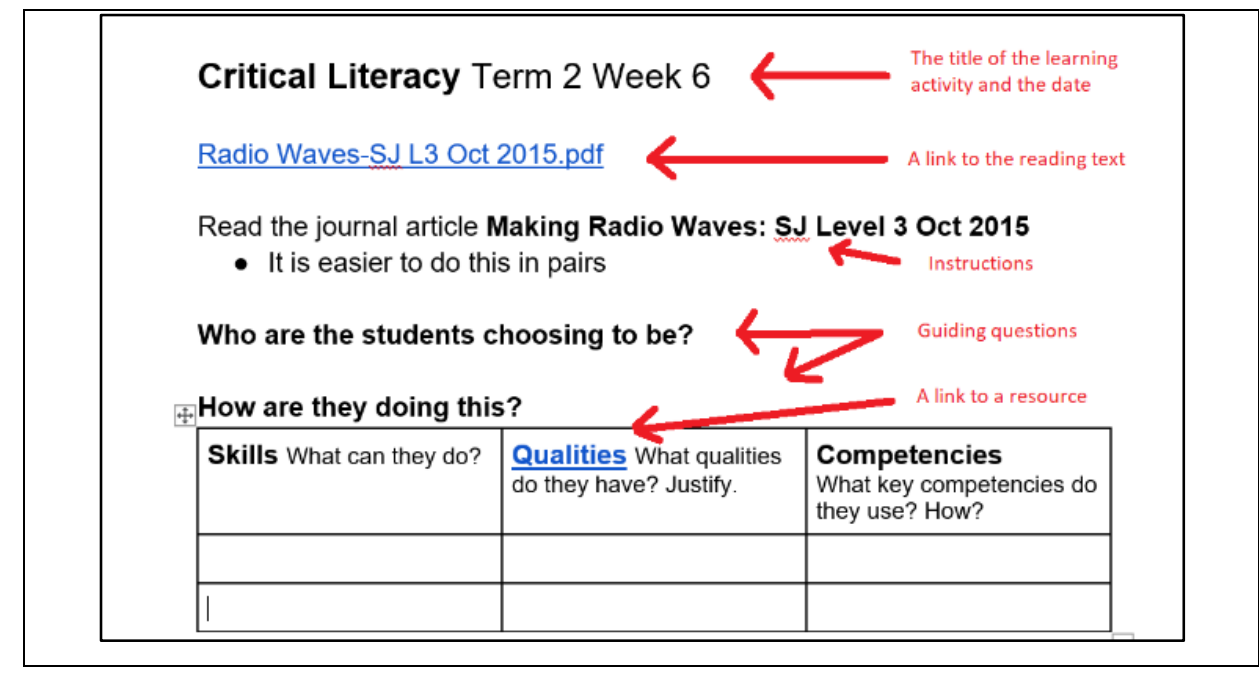

To evaluate and analyse the piece, the shared document included guiding questions and a link to a resource of definitions to help the students in answering the questions. They were also given a framework that included addressing skills, qualities, and key competencies. The questions varied based on the topic of the literacy piece, but the evaluative framework remained the same. The students usually worked on critical literacy in the library using their devices. They usually spent a one-hour session to complete and answer the documents, but sometimes they needed more time. Once the group completed the document, they shared it with their teacher for feedback.

'Cool Quick Write' was a literacy activity that required using the devices. Although it was not on my schedule to be observed, I had the opportunity to observe Sam while doing it. Kathy described the activity:

The idea of a Cool Quick Write is for the students to choose any text type they like. They do have access to examples of a range of text types. Using a prompt, sometimes a photo that they choose or one I provide that is linked to their inquiry, they just write without worrying about punctuation and spelling. We call it a 'dam burst' of ideas.

Kathy clarified the objective of this activity:

The objective is to share their ideas in an engaging way. They also have success criteria available that they have developed that establishes what makes writing engaging. They are given feedback about their writing from their peers about what 
makes it interesting and engaging and what they need to work on. They are required to choose one each term and craft it to go onto their learning blog.

Unlike the critical literacy activity, the students were required to do cool quick writing individually. In general, the students were given 20 minutes to do this activity three days a week before the time allocated to the workshops and the critical literacy.

\section{Summary}

In this classroom, three participants were observed from two year levels: Sam from Year 7, and Peach and Mark from Year 8. They were observed doing two learning activities: the first one was STEAM workshops; Music workshop, which was attended by Sam; 3D Modelling workshop, which was attended by Sam and Mark; Google Logo workshop, which was attended by Peach; and other workshops that were reported by Mark and Peach. The other learning activity was literacy where Sam, Peach, and Mark were observed doing critical literacy with their groups, and Sam was observed doing quick writing individually.

\section{Using Digital Devices During Learning Activities}

Six ways of use were inductively extracted: a source of information, a means for communication, a trial-and-error learning space, a production medium, an external personal memory, and a collective memory. There were general factors that shaped these uses and specific factors that shaped some uses in certain ways. In the following sections, I report first each way of use separately in conjunction with the factors that shaped it specifically, followed by showing the connections between uses. Then I present the general factors that shaped all the ways of use. As a nested complex system, the factors shaping using devices in the classroom were many and entangled. To capture these factors, I classified the factors in relation to the educational system, school system including classrooms, digital technologies, teachers, and students. To understand a complex system, acknowledging mutual interactions is as important as distinguishing them. Therefore, while I am distinguishing the factors shaping the uses, I describe how the factors influenced each other.

\section{A Source of Information}

Students used their digital devices to seek information during the observed learning activities. This use took two forms; first, a passive use where resources were sent to the students by the teachers or peers (Figure 4.3, below). Kathy shared with the students the timetable of the classroom. For the workshops, she shared the weekly information table about the workshops in Google docs. Mark and Peach were observed several times checking the timetable and 
workshop table before the workshops started. In the induction phase of the Music and Google Logo workshops, Peach and Sam accessed slides shared by the other teachers. These slides included the objectives of the workshops, main concepts related to the topic of the workshop, and links and sources to help students complete their projects such as tutorials and webpages.

In the literacy learning activity, teachers shared links to some sources such as reading text, videos, and information documents. During the observations, Mark was observed using the link to open the reading text to read with his group before working. Peach was observed opening the video several times to watch it with her peers. She also opened a document that contained definitions of the qualities sent by the teacher in an earlier time. On the top of Sam's 'Cool Quick Writes' document was a table that defined the types of writing and success criteria about what makes writing engaging shared by the teacher.

\section{Figure 4.3}

Screenshots of 'Passive' Use

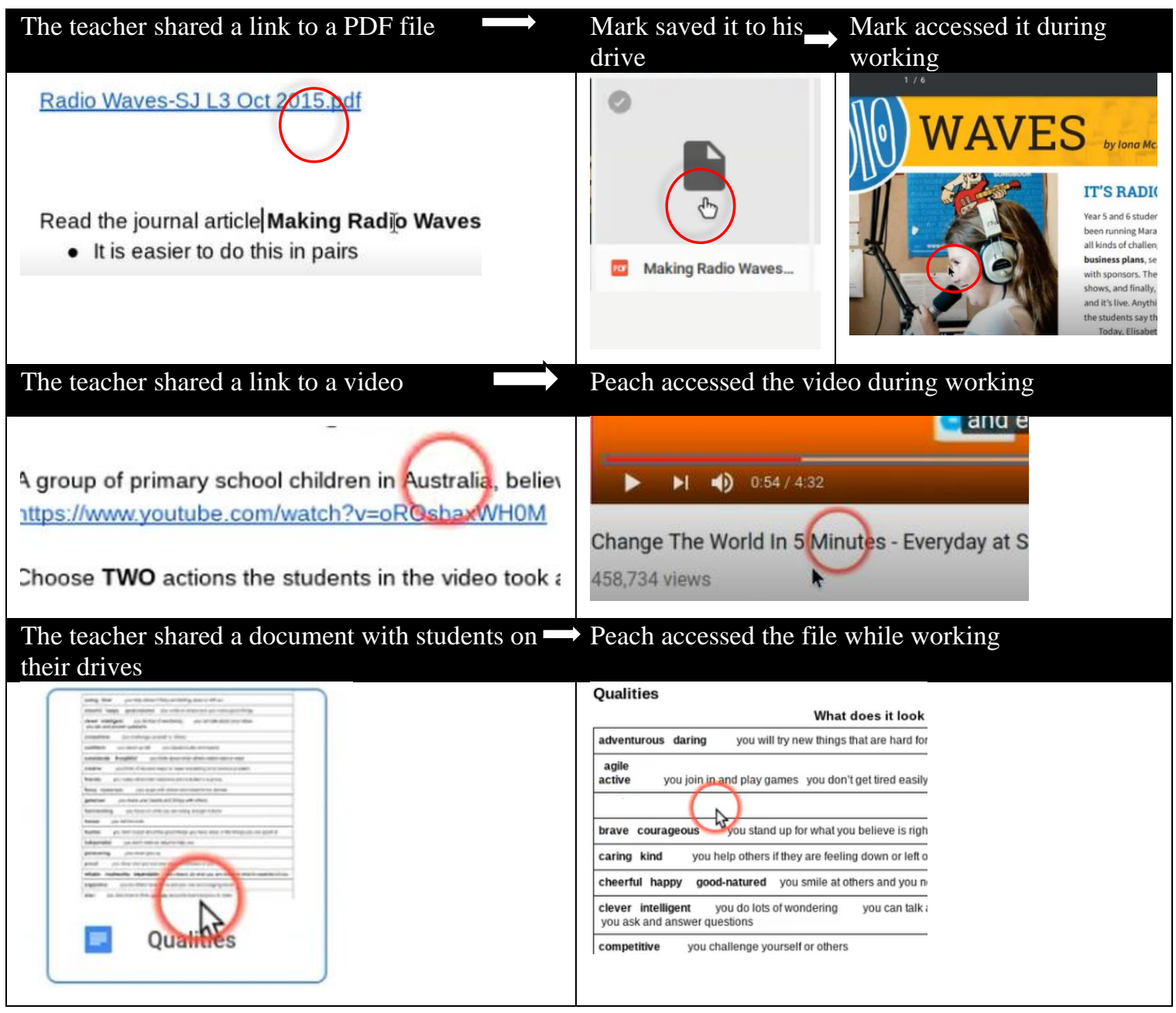


The second form of this use was interactive use, which happened when students searched for resources by themselves (Figure 4.4, below). For example, in the workshops, Sam used YouTube to look for some music videos and listened to them before working. Peach used Google search to look for images of a netball to draw one on her sketch paper before applying the design on her device. When she was looking at Google Logo samples about the lunar year, she wanted to know the meaning of 'lunar'. She asked the teacher, and the teacher suggested that Peach look for the meaning by herself. Peach used Google search to find the meaning and understand the concept behind the logo. In literacy, Mark used Google search to look for an image that illustrated the key competencies. He looked at different images then clicked on the one that was related to the New Zealand curriculum. Peach used Google search to look for a 'world problem' that she and her group were interested in, to make a change towards solving it. In general, most of the activities required passive use rather than interactive use.

\section{Figure 4.4}

\section{Screenshots of 'Interactive' Use}

\section{Mark searched for an illustration of the key competencies to evaluate a literacy text}

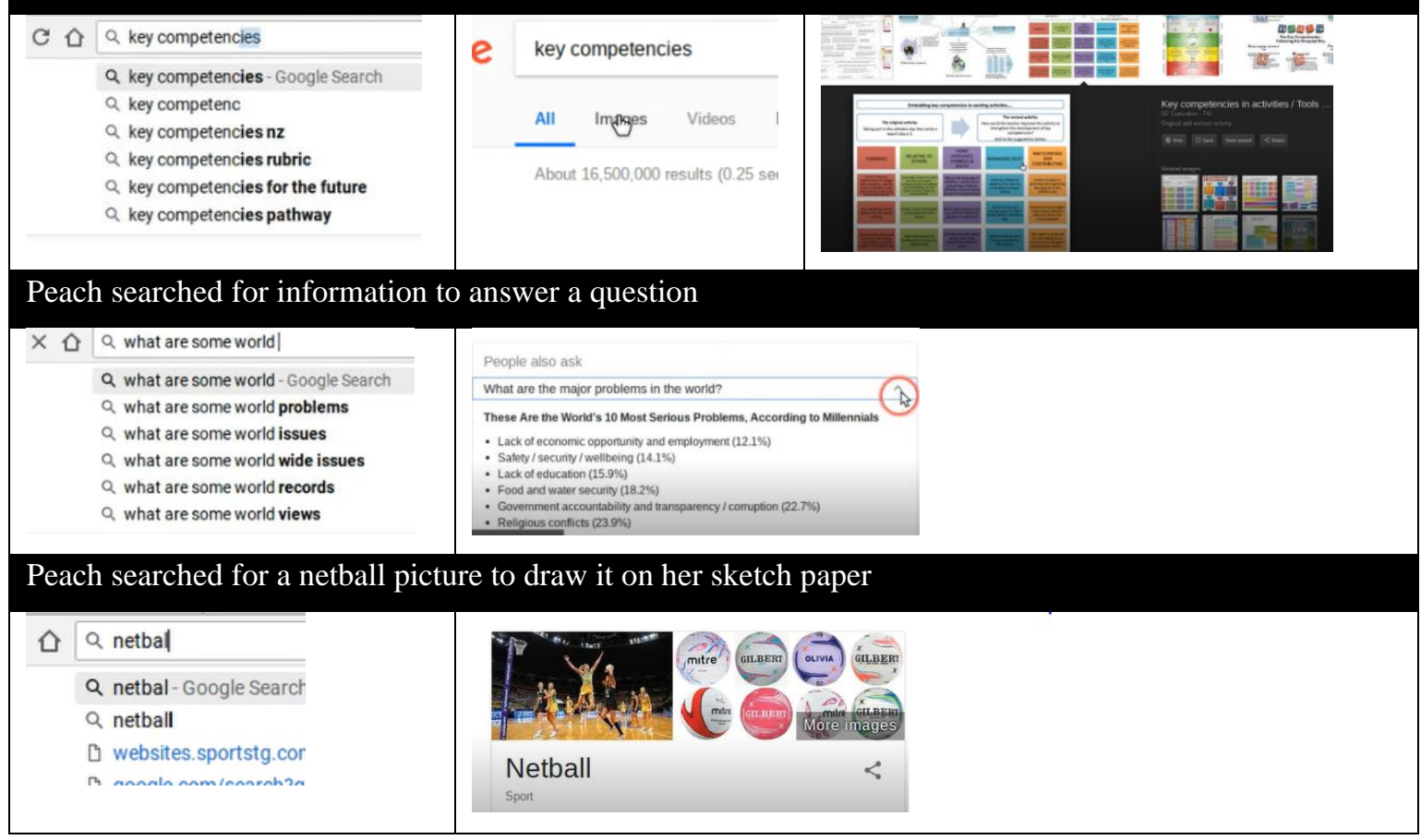

\section{Shaping Using Devices as a Source of Information}

Kathy mentioned that sometimes using the devices as a source of information might be more useful than other sources; she gave the following example: 
If it's something like being able to watch that video clip, which will actually give you a really good example of a young woman who's taking action and it's better than reading an article about her taking action, then that's worth doing.

Kathy predicted that her students would say, "I can access information and ideas so quickly and easily, I can very quickly find out any knowledge or facts that I need to find out". Kathy had knowledge of different digital sources and used them for different purposes. Therefore, using digital sources was encouraged in case the nature of the learning activities required searching for information, such as designing the logo, composing the music, or critical literacy. Kathy and the other teachers encouraged the students to search for answers for their questions. Additionally, teachers regularly shared digital sources with students, such as classroom timetables, slides, links, and articles.

Kathy's knowledge about her students was accurate. The students believed that digital devices were useful as a source of information; Sam said, "It's all there". This belief was accompanied by the participants' adequate digital skills. It was clear that the students were able to search and deal with different types and formats of sources, such as documents, videos, PDFs, websites, and images, smoothly and easily.

Digital technologies provided different resource and tools. They used search engines, particularly Google search, to find answers to their questions, illustrations of information, or samples of what they needed to do. In the interview, when the students were asked about other sources, Mark said, "I mostly use YouTube, but not that much”. Different websites were also used; Kathy gave examples, "we had access to ... science websites, educational websites". Other technologies enabled using the devices as a source of information such as Google drives, and Hyperlinks, which were frequently used by the students to find information.

\section{A Means of Communication}

Using digital devices as a means of communication took two forms; first, digital devices were used to communicate through it with others; and second, the participants used the devices as a topic or a reason to communicate about it with each other while doing the learning activities. Communicating about the devices, in this case, was to learn how to use the devices and to reflect on their use, while communicating through the devices was to get feedback or to contact with people from outside school. 
Communicating through the devices happened after the workshop activities were finished for feedback (Figure 4.5, below). The students wrote learning stories and shared them on their drives or blogs. Kathy, other teachers, and students wrote comments and notes to give feedback on these learning stories. In literacy, Kathy and other teachers' feedback was seen on students' assignments. The students said that their parents read their blogs at home and commented on their work. Kathy mentioned that Skype could be used to communicate with people outside the school, such as scientists, which enabled students to talk with experts and ask them questions. However, the feedback in Kathy's classroom was not limited to using digital devices. Mark said, while working on critical literacy, "they're [teachers] always checking it [the assignment] by the end of the day. It [using digital devices] wouldn't really make a difference because they're either checking it on the laptop or checking it by hand".

\section{Figure 4.5}

Communication Through Devices for Feedback Feedback on students' literacy assignments

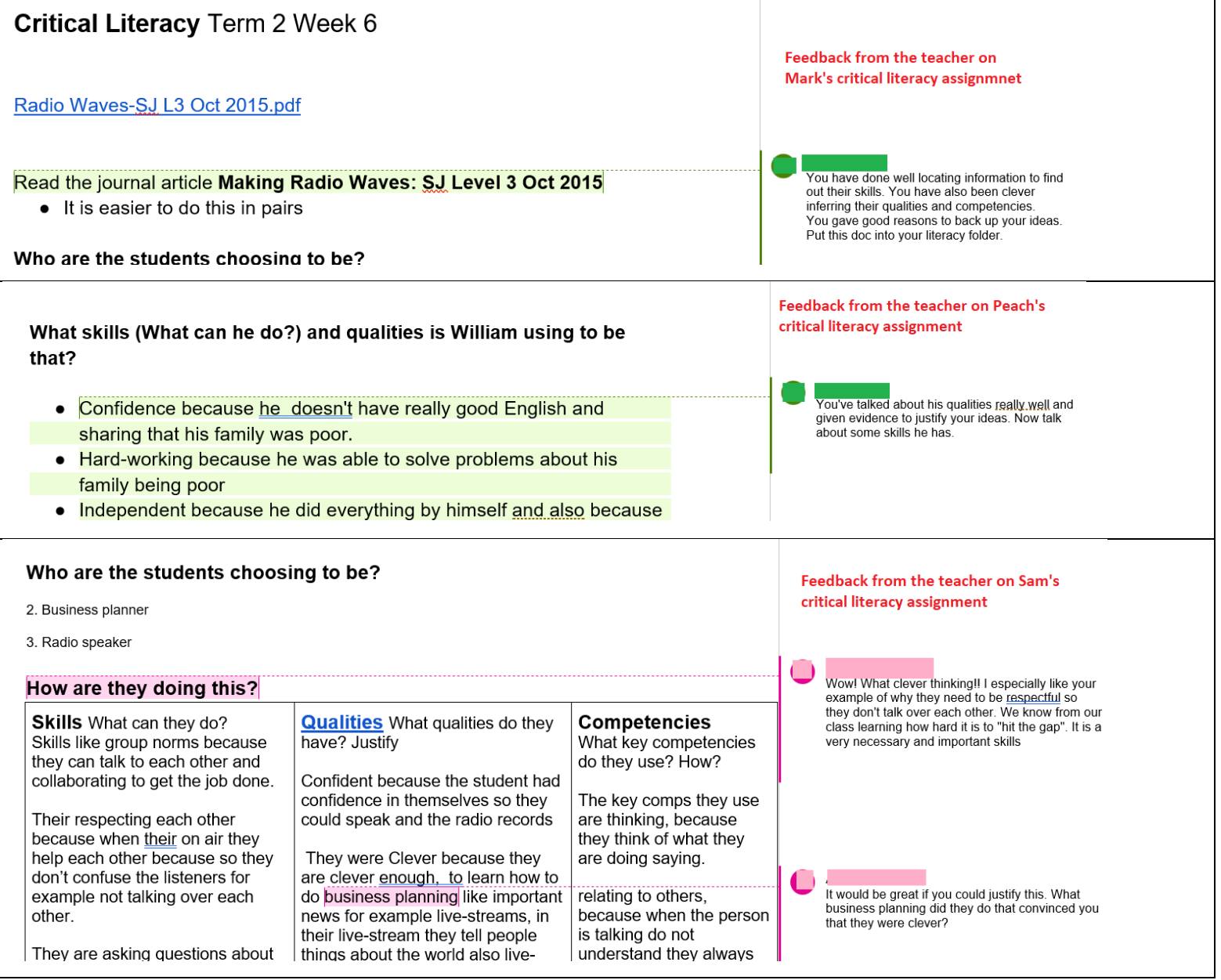


The students communicated about different topics while they were working together. They talked about the tasks, the assignments and the digital devices. For this study, I have focused on communication about the devices. These communications included how to use the devices, work on the applications, fix problems, and any discussion that the digital devices were related to or the reason for it. During the literacy learning activities, the participants did not communicate about the devices; the communication was basically about the assignments, while, during the workshops that I observed, communications about using digital devices occurred most of the time.

Using digital devices was a topic to communicate about what happened during the three phases of the workshops. In the induction phase, the teacher discussed many topics and concepts related to the workshops, and there was significant time allocated to talk about the devices. The students were not necessarily using the devices, but the dialogue was about them; particularly, why the students were going to use them and how.

In the implementation phase, the students actively communicated with each other. For example, the students regularly approached Mark to ask him about some functions and tools in the software. Peach was also approached by students to ask her questions about Scratch or to get her opinion about their designs. Even Sam, who was less technically competent compared to Mark and Peach, was very proud of himself when I saw him talking to one of his friends. He said, "I'm trying to fix something, a voice problem, I used to fix sound in my device". Although he did not fix the problem, he was happy that he had tried. Sam had intermittent conversations with his friends to ask them about how to do some functions or ask for their opinions.

In the evaluation phase, the students used the devices to share their projects; the whole phase was to discuss their learning experiences. The conversation aimed to discuss the success criteria and their evaluation of their performance and included talking about their experiences in using their devices. For example, Sam talked about taking risks by trying new features of the SoundTrap application; Peach expressed that digital design on Scratch was fun and she wished to do it again. The students were able to use their interactions with the devices as a tool, a reason, or a topic to communicate about it with others.

\section{Shaping Communications About and Through Devices}

Kathy encouraged the students to communicate about the devices either to share their learning experience or to help each other. She believed that "It's actually important that all 
our understandings have to be drawn out, and especially the expertise of the kids, and this is the whole thing around their confidence as learners, you need to hear from every kid". She added, "so it's actually those relationships are key, not only between the teacher and the child, but also between the members of the learning, all of the members of the learning community". She asserted in the interviews that students "learn best with each other", especially "when you have that rich conversation between the kids about how successful they are, about what they know, about what they can do, and supporting each other to do that learning". This explains the facilitation to communicate about the devices that happened during the implementation phase, as she believed that she needed "to facilitate the learning not dominate the learning".

Encouraging communications between the students, including communications about devices, was influenced by a professional development workshop that introduced the term 'relationships for learning'. Kathy said it was the "biggest single factor in students' achievement, and so it was how do we develop these relationships that are respectful, that recognise who the kids are?" The main pedagogical practice that reflected this was organising the students into mixed ability groups and encouraging the students to ask their peers even if they were working independently. These groups aimed to facilitate collaborative problemsolving. The students were required to share their knowledge and exchange their expertise about different learning areas, including using digital devices, Kathy said:

There's accountability. They know that, because they're working together, they all have to be sharing their gold. We call it the gold, they have to be sharing their gold. And so they also know that they're going to be coming down at the end in talking about the connect, you know, how we connect all our learning and they have to contribute.

Therefore, Kathy regularly reminded the students to collaborate and to help each other in solving the problems they encounter. The classroom environment and the classroom rules allowed the students, in general, to move freely or sit in groups to communicate with each other.

With regard to communication through the devices, Kathy believed the devices provided unique learning opportunities for the students:

You could talk to a scientist, you could skype a scientist. Well, obviously that's going to be so much better than reading a text about the scientists. You've got to find out 
what you want to know, what you're wondering. That's what this [digital devices] can allow.

Kathy said, "I think the most important thing for them is the idea that the world can access their thinking, so OK if the world can access it, that's very cool, and I can communicate with this massive range of people". She and other teachers regularly gave feedback through the devices. She valued feedback the students received when communicating through the devices:

If you put it on your blog, then so many other people can access. You've got a much bigger audience sharing your thinking. And so sharing your blog as much with your family, with your relations. Did you get to see the kids' blogs? So, you have. They have shared their learning with you and it's a really, really effective way.

There was an item in the 'Kawa of care' that regulated using the devices for communication that stated, "I will respect others by always using this device to interact with others in a kind, positive, and helpful way" and "I will make the most of this opportunity to learn lots of new things and to share these with others". Kathy reported students' commitment to this item and said that very few incidents had happened in school that were related to misconduct.

Students' digital knowledge and skills played a role in this use. Students who were known for their digital competencies, such as Mark and Peach, were approached by their friends many times during the learning activities to be asked about technical issues. Sam, who had moderate digital knowledge and skills, was regularly checking applications' features with his peers. He was able to offer help when he knew about some features, as reported in the communication section.

Students' attitudes towards collaborative versus individual work played another role. Mark, who preferred working with his peers, was regularly communicating while working. Sam and Peach were selective and preferred to communicate when they needed to or asked to. However, all of the students agreed on the importance of communication about learning with digital devices in the interview. Mark said that using digital devices was easy because you can learn from your peers, and Sam commented: "It's like what he [Mark] said, when you first turn on Chromebook and when other people help you, you can start using it". Peach added: "yes, I totally agree with them. People are really helpful because ... sometimes young people can help because they're like really young and go on technology almost every day". 
These attitudes towards communications about digital technology contributed to this use in the classroom.

The communication through the devices was influenced by students' self-esteem and confidence in both using the device and sharing ideas. Mark wrote on his blog:

I am good at sharing my ideas confidently and clearly because I want people to know what my ideas are. I need to work on making connections with other groups because mostly I like to do it my own way.

Kathy said, "They are really confident using the tool, they can communicate quite quickly with each other". Peach was reluctant to share her ideas in one of the sessions and said, "I don't have the confidence". To give and receive feedback the students valued using digital devices; Peach said, "It helps ... you can get feedback faster", which was agreed on by the other participants.

Several applications enabled communication through devices, such as Skype. The features of some application helped students to communicate through the devices. Sharing documents and an adding comments feature on shared documents or blogs allowed the students to communicate through the devices for feedback.

\section{A Trial-and-Error Learning Space}

Trial-and-error learning was observed when the students used their devices to try features or functions of an application or to test new ideas or designs for their projects. This use was observed frequently in the workshop learning activity and was not noticed during the literacy learning activities.

When the students were left alone with their devices in the implementation phase, trial-and-error was usually the first thing they started with. They used their devices to explore and try the features or functions of the applications, although they took different approaches to perform that. In the 3D Modelling workshop, Mark used trial-and-error learning on his device with his peers. He said that he had used 'SculptGL' software before but could not remember many features. He and his peers spent about five minutes trying and exploring different functions before they started designing the faces. They were stuck sometimes and spent time trying to overcome the challenge they were facing; for example, Mark did not know if the software had an 'undo' function or not and had to check the existence of this 
function. During this trial-and-error learning, Mark was engaged with the teacher and other students and shared their experiences with them.

Instead of doing the trial-and-error learning collaboratively, Sam preferred working individually. He explained, "I don't sit near my friends, because they distract me from my learning”. Thus, when he started designing the 3D faces in the 3D Modelling workshop, he sat in a quiet corner and worked individually, which happened in the Music workshop as well. When he started using trial-and-error learning, he was completely engaged and excited trying the functions and features of the program in a random, yet confident way. He did not hesitate to click commands as if he had been working on the software before. He liked to share his trials with Kathy and he said he felt better when she saw his attempts. Although he preferred working alone, sometimes he asked his friends about some functions or asked for their opinions about his trials. While he was doing the trial-and-error learning, he was smiling with no sign of boredom. When the teacher asked them to gather together at the end of the activity, he did not leave his device and kept trying the features. Sam spent the entire first session doing trial-and-error learning and said he would do his project at home.

Another reason for using trial-and-error learning on the devices was to enhance the work. In the Google Logo workshop, Peach's trial-and-error learning was not focused on learning new features or tools of the software as in Sam's case, but it was focused on trying different designs for the logo. The reason was that Peach was familiar with Scratch software and had used it before. Peach started her attempts by sketching some designs on paper, as suggested by the teacher. Then, when her initial sketch was completed, she started trying it on her device. While designing the logo, she had to use the 'undo' function frequently. She tried different features, some of which were new for her, and designs before she decided how the logo should look. She said it was easier for her to make changes to her design using the device although she preferred using the pencil. Peach worked alone and quietly although she was interrupted sometimes by other students asking about some functions or asking for her opinion.

The three participants did their trial-and-error learning on their devices in different ways. Mark did it collaboratively; his trial-and-error learning included continuously interacting with his peers. Sam preferred doing it alone most of the time, but when he needed help, he asked his teacher or some of his peers. Peach spent most of the time doing this learning independently and used paper and pencil in her trials. However, all of them were 
active, engaged, and focused on the task. In all observed workshops, the students were never reluctant to try new things or worried about making mistakes regardless of the type of application they used.

\section{Shaping Trial-and-Error Learning}

Kathy used different teaching strategies to reinforce this use. There were explicit instructions such as, 'explore!', 'try [a] new thing', or 'take risks'. Some of these instructions were displayed on the projector and discussed with the students as in the Music workshop or verbally confirmed by the teacher as in the 3D Modelling and Google Logo workshops. For example, in the Music workshop, one of the slides presented to students stated, "Explore SoundTrap: Tip: Get the most out of SoundTrap by taking a look at the tutorials, e.g. crash course". The nature of the learning activity also played a role. Trial-and-error learning was observed in the activities that focused on developing digital skills, such as the Google logo or 3D Modelling workshops, while it was not observed in the literacy activity where the focus was on evaluating literacy.

Different functions and tools in the application enabled the students to explore and try things before and during creating their digital products (Figure 4.6, below). One of the features that seemed to be significant for trial-and-error learning was the 'undo' function. Mark, for example, had to re-start his design all over again until he was able to find the function. In general, the students were not afraid of making mistakes while working on their devices, as the applications were flexible enough to give the students the opportunity to explore and modify their work.

\section{Figure 4.6}

Screenshots of Features Used During the Trial-and-error Learning

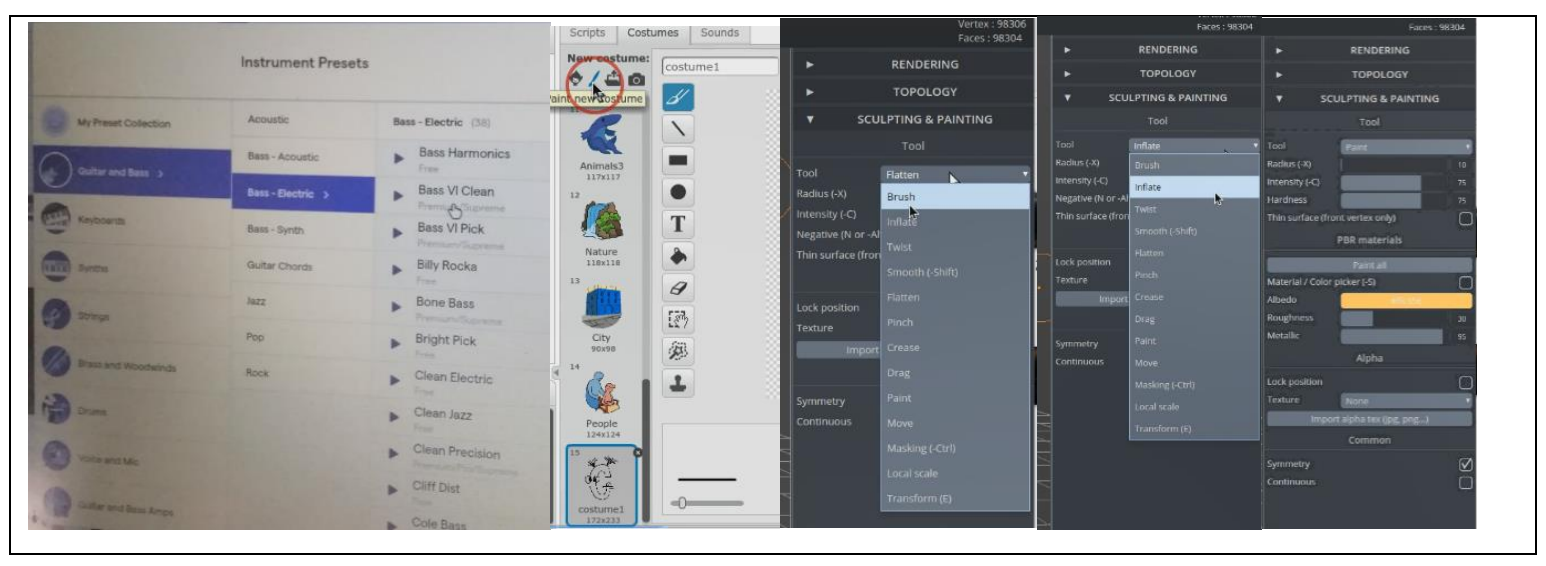


When comparing how the three participants used the devices for trial-and-error learning, their digital skills influenced the time spent on this use and how effective it was. Peach, who had previous knowledge about Scratch spent less time because she reported that she knew most of the features. Mark did not spend a long time in the trial-and-error learning and was able to start doing his project quickly, although he was absent in the first session. $\mathrm{He}$ said he could not remember many features of the software, but he could link most of these features to other software he was already familiar with. On the other hand, Sam spent a long time, as the software was new for him.

\section{A Production Medium}

The students used the devices as a medium to produce digital artefacts, such as documents, assignments, blogs, designs, and music. For STEAM workshops, the participants were required to create a product, depending on the topic of the workshop. The students worked either collectively or individually, but each student was expected to create his or her own product. In the critical literacy learning activity the students were required to work in groups and submit an assignment that included their evaluation of a text or video collectively. Students' blogs were also produced by the students individually and were used to record their learning stories in the workshop learning activity alongside other learning activities.

In STEAM workshops, using the device as a production medium was related to trialand-error usage. They occurred simultaneously during the implementation phase in the first and second sessions, and they depended on each other. Peach created a Google logo using Scratch software. As she was familiar with the software, she quickly started the design process. She was competent in using the software; she was fast, knew many functions, and used them efficiently. She persevered until she finished the symbol and completed most of the work in the limited time given in the second session.

Mark, who was absent in the first session of the 3D Modelling workshop, was able to design different 3D images using SculptGL software in the second session. He worked collaboratively with other students. He showed excellent technical skills while working; he worked fast, used many tools, and knew how to fix problems. Sam, who was also familiar with the software, needed more time to design fewer 3D images in a session. In the Music workshop, Sam used SoundTrap software for the first time. He spent most of the implementation phase doing trial-and-error learning and said he would complete his project at home and continue in the second session. Students' digital products were saved on their 
accounts on Google Drives and shared with teachers and peers for feedback in the evaluation phase.

In the critical literacy activity, the students received a weekly template from their teacher to write an evaluation of a text or a video clip collaboratively. The template differed from week to week, and the content differed from group to group (Figure 4.7, below). Peach was the one who typed the evaluation in her group, as she was the fastest according to her peers. While typing, Peach frequently used the spell check function; for example, she used the spell check function seven times during 10 minutes of typing. Sometimes she directly corrected the words underlined with red, and sometimes she opened the words' suggestions and clicked on the correct word. She used the copy-and-paste function during her work. Peach was competent when using the editing tools; she was fast and knew the features and tools to do the assignment.

On the other hand, Mark was not the only one who was typing on the assignment; he and another two peers were typing at the same time. Mark was fast, accurate, and did not have misspelt words during my observations.

Sam's contribution in his group was sharing ideas and discussion, not typing; his group chose the fastest student to type. For this reason, one observation of Sam was conducted while he was doing the 'Cool Quick Writes' learning activity to explore how he used his device to produce a digital artefact for literacy. Sam's piece of writing was a dialogue that told a story; each week he wrote a section of the story in a 20-minute session. Sam's word count ranged between 87 to 752 words for each session. His story included different fonts, pictures, capital and lower case letters, and punctuation marks such as “!”, “,”, “?”, “.”, and “:”. During the observation, Sam used the spell check function but not for all words. The reason was that the students had limited time to finish and, as Kathy mentioned in the interview, the focus in the 20-minute session should be on writing not on the language features which would come in a later phase. 


\section{Figure 4.7}

Samples of Students' Critical Literacy Assignments

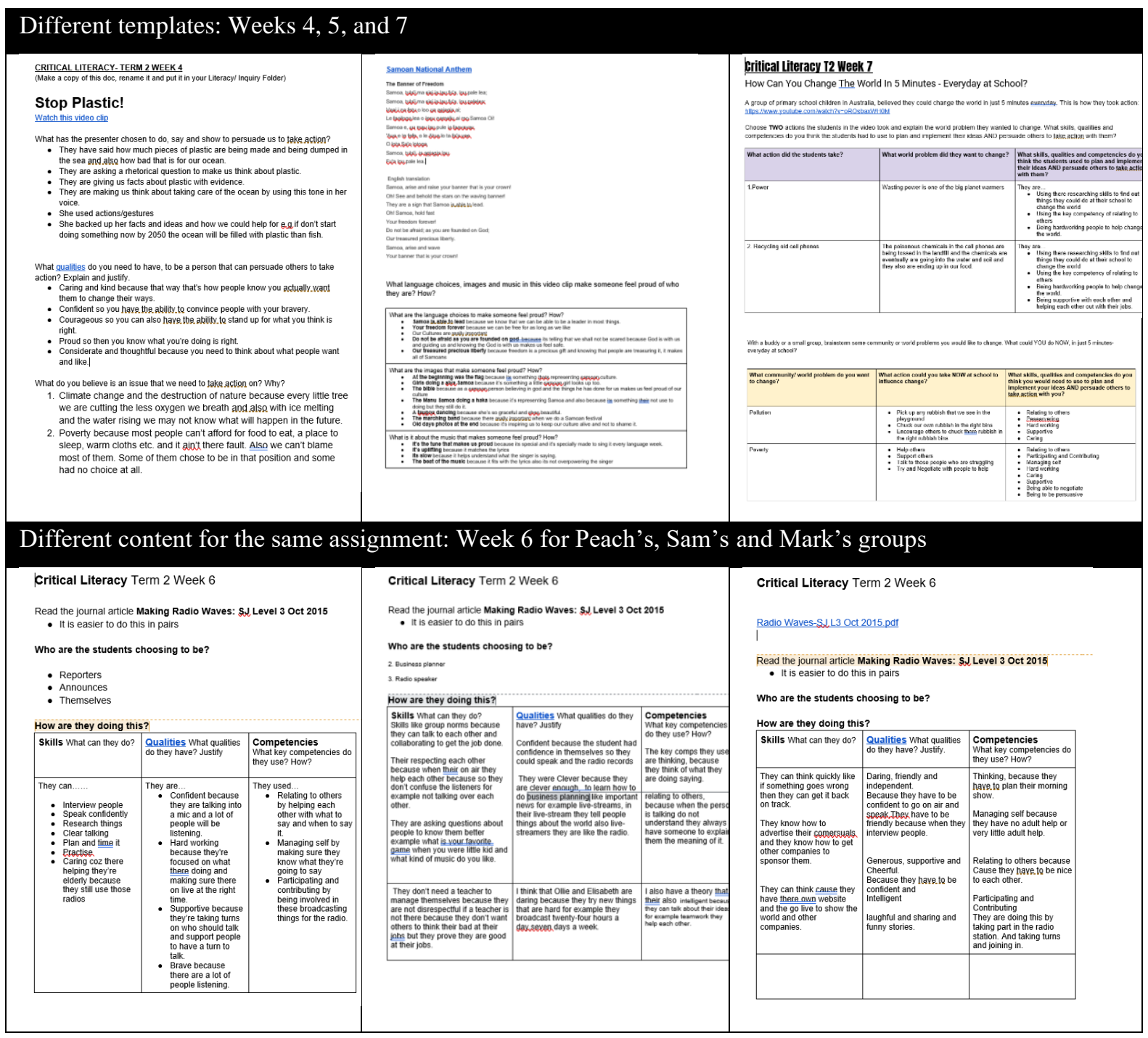

\section{Shaping Using Devices for Production}

Kathy engaged students' culture, learning framework, and teaching strategies all together which influenced using the devices as a production medium. Kathy believed that it was important to embed students' culture in their learning and what they produce, to keep them engaged and see the learning as relevant and worthwhile. Her understanding of 'culture' was broad:

[Students learn best] in a setting which they can see the purpose for what they're doing, that has a cultural context that they can relate to, and when I say "culture" that's actually really broad. It is not just ethnicity, so it might be they recognise the family culture, that may be happening in a classroom, or they recognise the youth 
culture. So it's something where they say: I can see myself as a learner in this context because I belong and because there is an indication that I'm understood, who I am is understood.

That influenced her choices of the digital products that the students were expected to create. Kathy, for example, used the Samoan language to describe faces in the 3D Modelling workshop and the Samoan National Anthem for critical literacy assignments, as many of her students were Samoan. In the Google Logo workshop, the students were required to design a logo that represented their identity. This shows Kathy's pedagogical knowledge about learners and context.

Kathy used the success criteria and the key competencies as frameworks to guide students' production processes. The success criteria and what exactly was required from the students to do were discussed in detail by the teacher in the induction phase and the students participated in creating them. Kathy said:

I think that's actually what makes really successful learning is they [the students] are invested in the learning because they've come up with the criteria that make them successful and then they say, personally, I can talk about how successful I was at that. I know I need to work on that.

Peach wrote, commenting on the graphic design workshop, which was not observed, "This workshop involves a lot of thinking and relating to others. Our purpose was to learn new skills and techniques that we think we might need to learn how to draw". This shows that the participants were taking the success criteria and the key competencies into account while creating their projects.

In the evaluation phase, the students used the criteria to evaluate and reflect on learning. For example, in the Music workshop; the success criteria were displayed on the projector and shared with the students (Figure 4.8, below). In the induction phase of the Google Logo workshop, Peach shared her opinion about the success criteria of the logo and said, "it has to be engaging, colours should be chosen carefully to represent your identity". This shows that success criteria shaped the production process of digital artefacts. 


\section{Figure 4.8}

Success Criteria in the Music Workshop

What will success look like?

- When we have created a piece of music that creates an emotion (describing the sound).

Kathy knew different applications that the students could use for their learning. However, she believed that using devices for production was not always the best option: "if it's just easy for you to write down than it is for you to go like that [typing] then you don't need to go like that [typing]"; this aligns with SAMR model that she adopted. She said, "In literacy, the Chromebook will only be helpful for, maybe word processing, but in lots of our workshops there're apps and things that they can use". Kathy learnt about some of those applications in professional development workshops and said, "the challenges for me will always be how to use the device effectively and how to support kids to use that device effectively". She added, "If I knew about apps and websites more, then I would know more possibilities especially in terms of their learning”. For this reason, Kathy collaborated with other teachers who had advanced digital skills to run the workshops when needed.

Kathy used various teaching strategies that influenced the production process. In the workshops, she gave detailed and step-by-step instructions to guide students' work. In the Music and Google Logo workshops' induction phases, the what to produce was explicitly displayed on the projector and shared with the students on their Google Drives. The instructions stated, "Create: Choose a video clip below and design a piece of music to accompany the footage" then added, "Create a Google sign that tells a story about yourself". In the 3D Modelling workshop, Kathy explained that students had to create 3D images of different facial expressions. In literacy, the students were given templates, which varied from week to another. These templates included instructions of what to write and what questions to answer, and the students were not required to make changes on the design of the templates.

In addition, Kathy used samples to illustrate how the final product might look. Different types of samples were used and included digital and non-digital samples (Figure 4.9, below). In the 3D Modelling Workshop, Kathy used a hand-made poster illustrating the expected designs of the facial expressions. Mark and Sam kept looking at them to check their designs while 
working. In the Google Logo workshop and the Music workshop, the samples were digital. Peach liked the musical symbols in the digital sample and started her work by imitating the idea, but later she changed her mind and preferred to design a symbol that reflected her artistic side.

Online tutorials were used to give students detailed step-by-step procedures. In the Music and Google Logo workshops, one of the tutorials was first displayed in the induction phase, then the links of other tutorials were shared with the students. Sam used these tutorials a few times, while Peach just looked at them once. In the 3D Modelling workshop, step-bystep instructions were not used because the students already had previous experience of using the software.

Group work as a pedagogical practice also influenced students' production of digital artefacts. In literacy, students were required to work and submit their work collaboratively. While in workshops, the students produced their work individually but had to collaborate with each other.

\section{Figure 4.9}

Teachers' Samples and Students' Products in STEAM Workshops

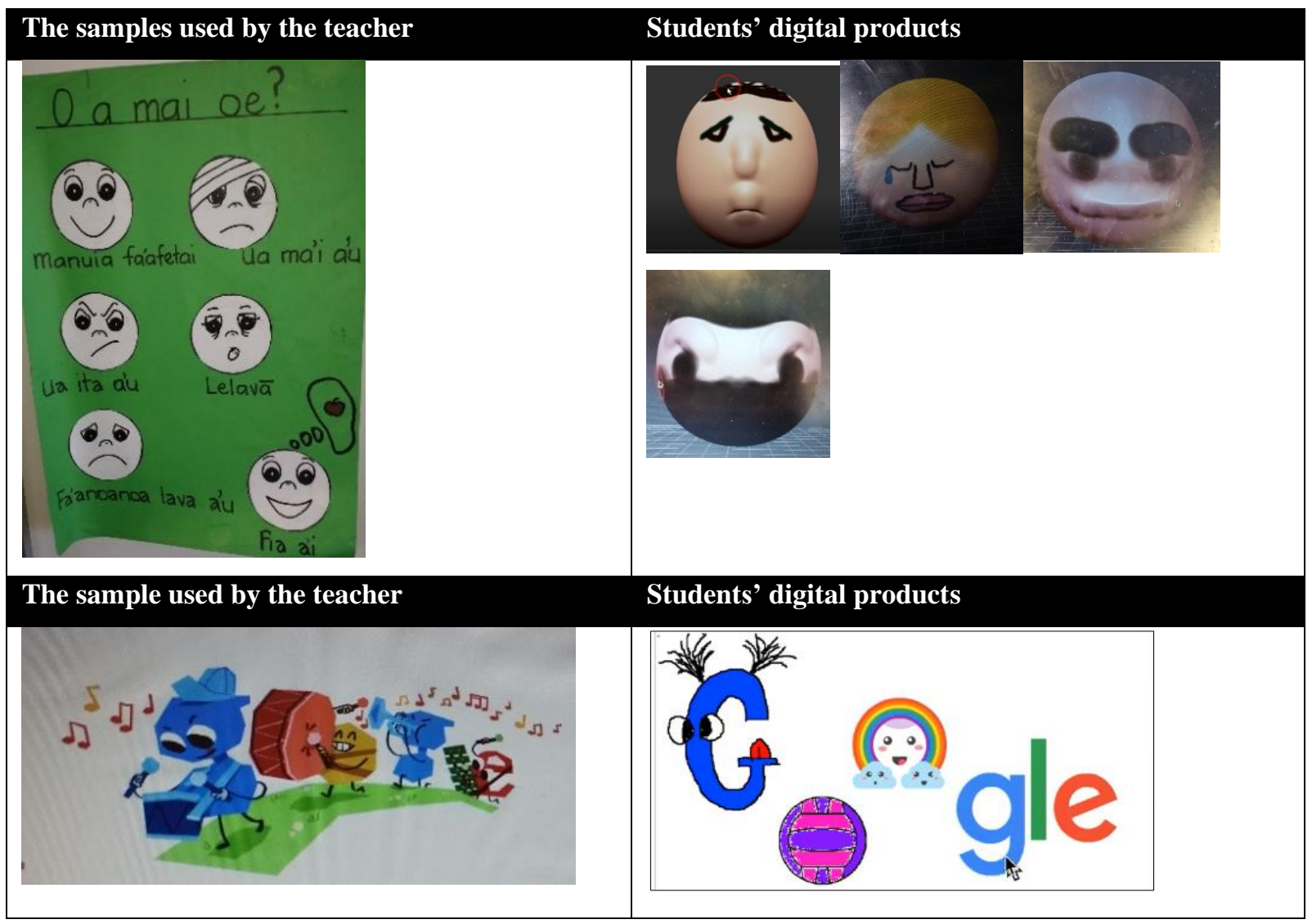


A wide range of applications with different features were used for digital productions, such as word processing, music, 3D design, and coding software. Word processing features and editing tools were used frequently by the students, including the spell check function. Peach's final draft of the literacy assignment was free of misspelt words, just like Mark's draft. It was interesting to notice that Peach regularly used the spell check function while typing, while Mark rarely used it. That shows that some of the functions enabled good quality digital products regardless of students' capabilities. Additionally, the tutorials of the applications were helpful, as discussed. There was a limitation reported by Peach, who said that using paper and pencil were much more flexible for her than using the mouse for designing the logo and said, "what a mess! this would be easier if I could draw it with my hands with pencils". In general, the participants in the interview agreed that digital devices were helpful and made doing things easier.

Students' digital skills and knowledge influenced the production process. As reported, Mark and Peach showed competencies while using different applications. They knew different features and tools; they used bulleted lists and numbered lists, bold and regular fonts, different fonts and colours, and added pictures. They were able to solve some technical problems as well. In general, Kathy praised her students' digital skills:

They can! Once they do something, they can just find it and do it really easily and they can find really awesome ways of doing it ... some of those apps that we use in the workshops ... They use apps confidently. Yeah, they're always like that.

Students had different preferences. Sam wrote about himself: "The workshop this boy wants to be in is music because music has been a type of life for his family". Peach wrote about another workshop needed using the devices: "I really enjoy it because Comic and Graphic Novels has given me a chance to express my artistic side. It's been a fun experience”. Her logo reflected her preferences. Mark wrote, "I did this workshop because I enjoy learning about technology, and I enjoy using it. The purpose of this workshop was to shed new knowledge to kids like me". That shows that students' preferences influenced their choices of the workshops and digital products that they expected to use.

Students' preferences led them to sometimes not use their devices. Kathy said, "some of our digital natives with the Chromebooks choose to use a piece of paper". She added, “There might be a group says that we don't need the devices to solve, but there might be another group who says we really, really need the device to solve this problem". 


\section{An External Personal Memory}

In this study, external personal memory refers to the process of saving and retrieving digital artefacts, either created by the students or collected from the Internet, in digital storage spaces. They were personal because only the student had full control of what to save and who could access the content. They were external because the items were saved in places outside their brains, such as on drives, blogs, or accounts.

The three participants had personal Google Drive accounts. Regularly, they saved their work created for schoolwork and information they collected. They saved their achievements during the implementation phase; Sam saved the piece of music he had composed on his SoundTrap account. Mark and Sam saved the faces they had designed on their drives during the 3D Modelling workshop, while Peach saved her Google logo design on her Scratch account and pictures she collected from the Internet. Another example of the saved items was learning stories, which included their memories, thoughts, reflections on their learning experience, and sometimes pictures. Students saved these stories on their drives or posted them on blogs. These blogs were private and needed permission to be accessed; the students permitted their teachers, peers, families, and others, like me, to access the content of these blogs to share and get feedback. Consequently, these blogs became an external personal memory for each student. Another way of recording students' thoughts was the 'Cool Quick Write', which Sam was observed doing for literacy. Kathy described it: "it's called 'Cool Quick Write' because you just really quickly recording your thinking, and then you can craft it".

In addition, the students saved some documents in their accounts that they did not create. An example of this was observed during the critical literacy learning activity. Peach was trying to answer the question of "What skills, qualities and competencies do you think the students in the article used to plan and implement their ideas AND persuade others to take action with them?" She first wrote two sentences then spoke aloud to herself "What other qualities?" She then opened her drive to check a saved document that explained the key competencies. She checked the document then found another competency that could be added to answer the question. Peach commented that it was quick to find what she was looking for when using digital devices.

Another way of using the devices as an external memory was observed when Peach wanted to design her logo. She used a mind map saved on her account of an application 
called Mindomo (Expert Software Applications srl, 2018). This mind map was created in a previous learning activity and contained several nodes about herself, such as her personality, interests, and talents (Figure 4.10, below). Using the mind map was surprising for Kathy, who did not expect the students would refer to their previous work.

\section{Shaping Using the Devices as Personal Memory}

The students signed a 'Kawa of care' item that stated, "I will protect my password and keep it completely secret". Kathy reported that the students were committed to it. Kathy believed that her students would prefer recording their stuff using their devices: "for lots of them [the students] it's an easier way of recording". She added, "they can Screencastify or they can record things ... they've got really good records I guess of their learning and thinking”. Kathy reported that they taught their students how to manage their personal accounts previously:

I should jump back and say that we had to have some really structured teaching around: "this is how you organise your drive", "this is how you do this", "this is how you do that". And so that was quite structured.

\section{Figure 4.10}

\section{Peach's Mind Map}

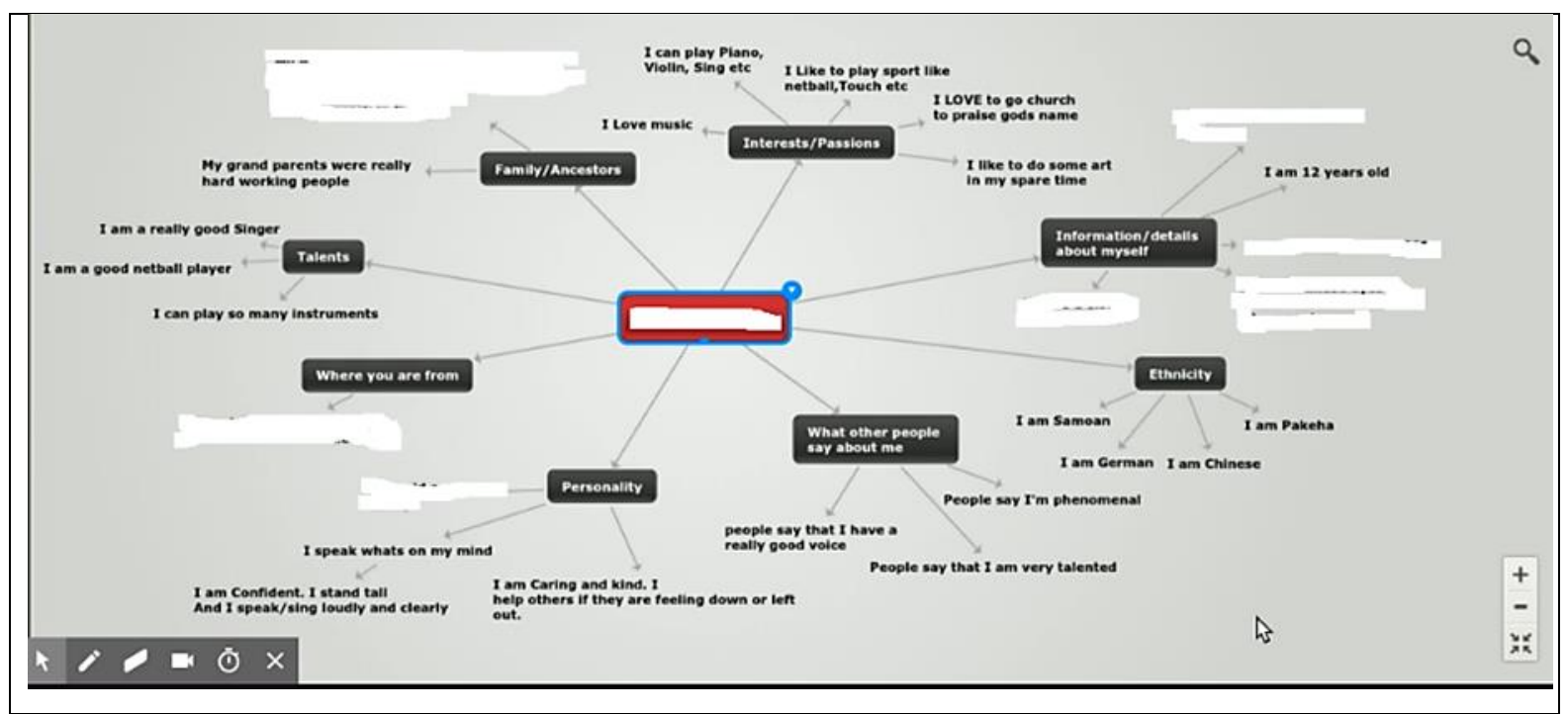

There were detailed instructions about how to record students' learning stories and organise students' Google Drives written on the classroom whiteboard, so students could read them anytime (Figure 4.11, below). 


\section{Figure 4.11}

Detailed Instructions about Saving and Recording Students ' Work

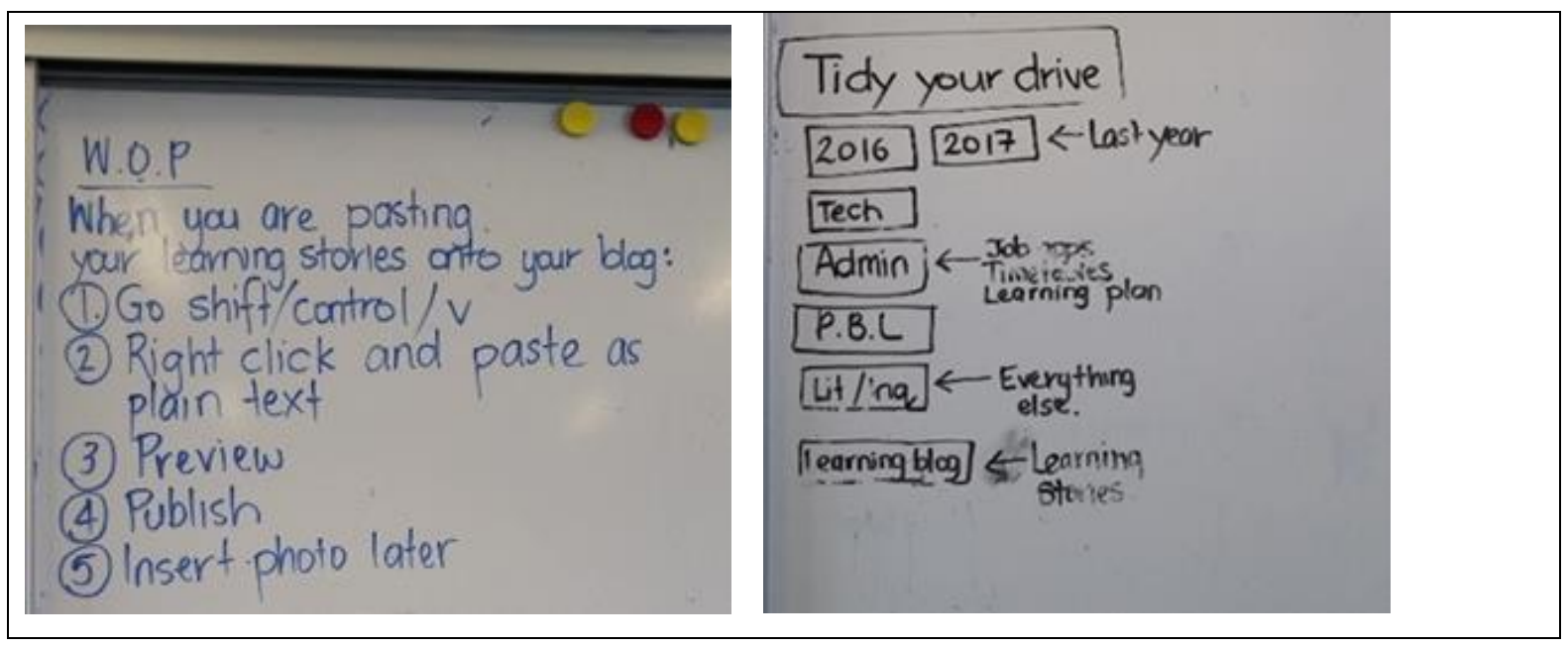

Kathy used pedagogical strategies that required creating personal memories. These strategies were learning stories and 'Cool Quick Write'. Unlike the 'Cool Quick Write', which required quick recording of students' ideas, learning stories should be crafted; Kathy explained:

The students only really recorded learning stories about their learning on their blogs.

These were crafted learning stories that had to be edited and proofread before they were put onto the blog. The students had a clear understanding that anything on the blog that others had access to, needed to be error-free.

In the learning activities, the students were expected to save their work as a prerequest to other tasks, even if the instructions did not state that explicitly, for example, saving their designs, assignments, and music to be sharable for feedback and review.

These efforts influenced students' competency in dealing with their accounts. The three participants showed good digital skills related to saving, retrieving, and managing their work smoothly and quickly. Their drive accounts were organised into folders and none of them needed help to access these accounts during my observations. The students were very careful and precise when sharing their saved work with me, which reflects good management skills of their online accounts.

Most of the applications used allowed the students to have personal accounts with passwords. The storage limit was one of the features that concerned the participants. When I 
first asked them to share with me their recordings on Screencastify they expressed concerns about the storage limit of the free account, while they were more relaxed about this issue when dealing with their Google Drives or blog accounts. The other feature was the privacy feature of the account, which allowed the students to control who could access their work and who could not.

\section{A Collective Memories Coordinator}

The students worked collaboratively through the devices to create digital artefacts such as documents or assignments. These documents became a collective memory when the students saved them in a shared folder and all the members of the group could access and retrieve them when needed. For example, in Marks' drive (Figure 4.12, below) were shared documents, which could be accessed by him and other students alongside his personal documents.

\section{Figure 4.12}

\section{Mark's Drive Account}

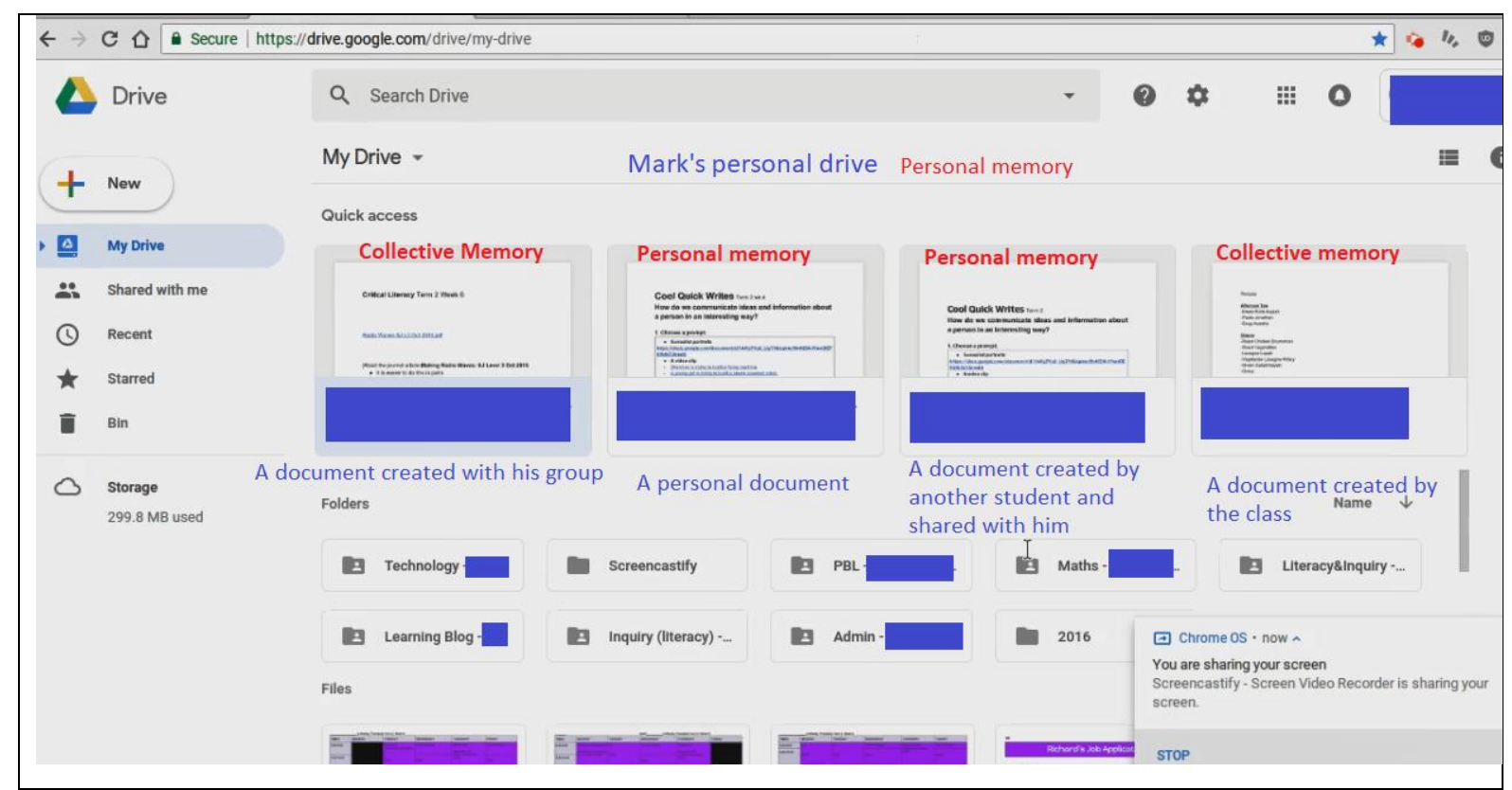

An example of creating and using collective memory was observed in the induction phase of the Google Logo workshop. Peach and the group were asked to use their devices to open a shared document on their Google Drive, which was also displayed on the board by the projector. That document contained different digital samples of Google Logos, and the students had to write their comments and reflections on these logos. In the beginning, Peach 
typed a few words commenting on a logo that interested her, then she wrote more sentences about what the logo told her, the reasons for choosing this logo, and what made that logo attractive. While working, she read some of her friends' comments and corrected their spelling before the whole group discussion. By the end, there was a document that recorded students' ideas, reflections, and opinions. It was saved on a shared folder that could be accessed and modified by the whole group. Peach opened it once when she started her work.

In the critical literacy activity, this type of use happened frequently. As mentioned, every week, the teacher sent a document to the students' groups to evaluate a piece of work. As the critical literacy activity was a group work activity, the students worked together to complete the task. During the observations, the groups used different ways of creating these documents. In Mark's group, Mark and his friends were typing on the same document simultaneously (Figure 4.13, below) while discussing what each one of them would write. Once they were finished, Mark and his peers reviewed what they wrote; when all of them agreed, the students shared it with their teacher for feedback. Then they shared it with me for the research.

\section{Figure 4.13}

Mark when Typing Simultaneously with his Friend

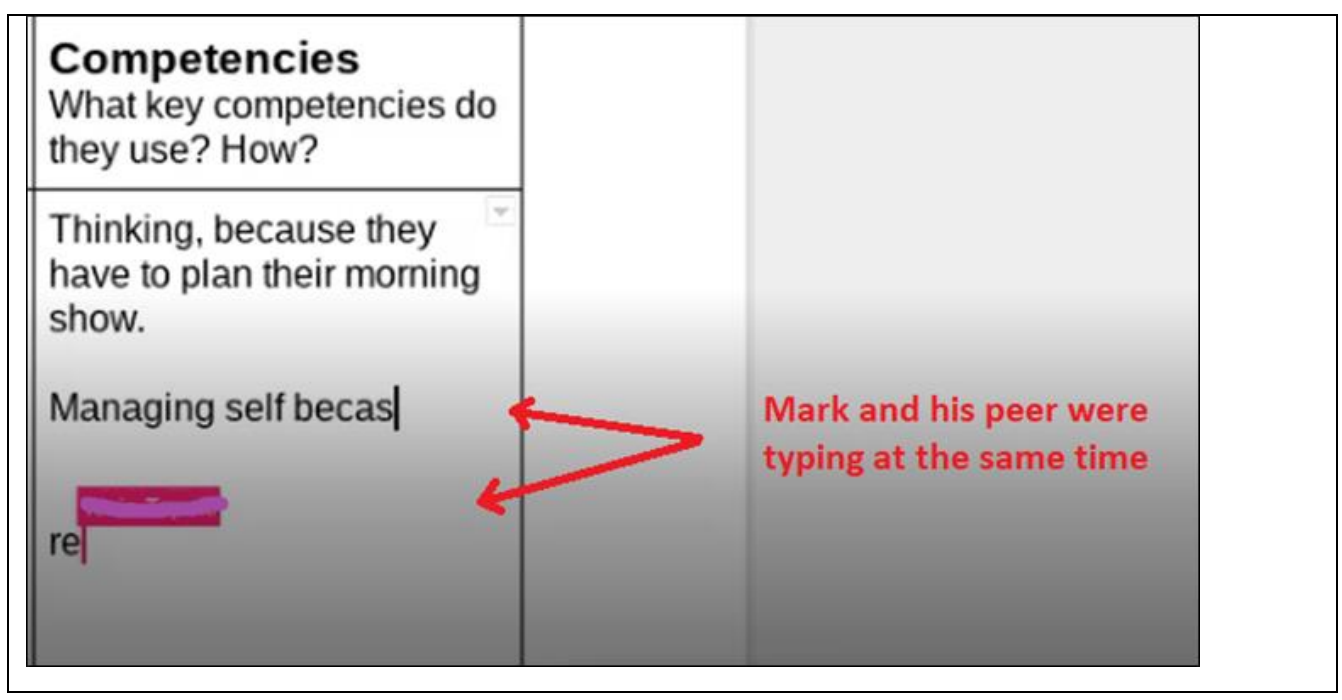

In Peach's group, the creation of this shared memory was different. Peach was the only one who was typing. Peach and her peers discussed first what to write before she recorded their ideas on the document. Sam participated in the discussion, and other students recorded his ideas. Sometimes, the students retrieved documents when doing their tasks. For 
example, while Peach and her group were thinking of ideas to add to their answers, Peach opened an old assignment to compare it with the new one.

\section{Shaping Creating Collective Memories}

Creating collective memories in the classroom required the students to work collaboratively. Kathy believed in the importance of collaborative work for students' learning. She described how she and the other teachers worked to make sure that students' groups worked effectively together:

[We've] been really clear about how to develop the group norms so that those kids actually do talk with each other and say: "oh, I disagree with it" or "yeah, I agree with you because" or "what do you think", you know, all of those things. We collaborate and we make sure that those are in place; because if the groups can't work together, if the kids can't communicate their thinking, that sort of learning is going to be much less than they would.

In addition, the teachers participated with the students in creating and accessing collective memories, as described in the previous section.

Kathy's approach influenced students' behaviours. Their collaborative work to create collective memories was well organised and went smoothly. Still, there were differences in how collective memories were created. In Sam's and Peach's groups, students who were fast in typing were responsible for recording the ideas for the group members. In contrast, all the members in Mark's group participated in creating their document. That shows that the way the students collaborated and delegated tasks influenced this use.

Collaborative digital tools, such as shared folders, were a key to this type of use. Kathy talked about the importance of these features for collaborative problem solving:

[There's] just a range of apps that really allow for some really good problem solving, these apps that they can choose to use, where they [are] actually working collaboratively to try and solve quite simple, quite complex problems ... it's just such a good tool.

That included simultaneous access while creating the same file, automatic saving, and shared features that enabled users to access the saved files after working. 


\section{Connections Between Uses}

There were connections between the six ways of use in this study. The connection means that the use triggered other uses to happen or was triggered by other uses. Based on the description above, Table 4.1, below, summarises these connections.

\section{Table 4.1}

Connections Between Uses in Case One

\begin{tabular}{|c|c|c|c|c|c|}
\hline & $\begin{array}{c}\text { Source of } \\
\text { information }\end{array}$ & & & & \\
\hline $\begin{array}{l}\text { Means of } \\
\text { communication }\end{array}$ & $\begin{array}{l}\text { Students } \\
\text { communicated } \\
\text { through the } \\
\text { devices to receive } \\
\text { or share } \\
\text { information }\end{array}$ & $\begin{array}{c}\text { Means of } \\
\text { communication }\end{array}$ & & & \\
\hline $\begin{array}{l}\text { Production } \\
\text { medium }\end{array}$ & $\begin{array}{l}\text { Students searched } \\
\text { for information to } \\
\text { create their digital } \\
\text { products }\end{array}$ & $\begin{array}{l}\text { Students } \\
\text { communicated } \\
\text { through the devices } \\
\text { about their products } \\
\text { and communicated } \\
\text { about using the } \\
\text { devices to create } \\
\text { their products }\end{array}$ & $\begin{array}{c}\begin{array}{c}\text { Production } \\
\text { medium }\end{array} \\
\end{array}$ & & \\
\hline $\begin{array}{l}\text { External } \\
\text { personal } \\
\text { memory }\end{array}$ & $\begin{array}{l}\text { Students used } \\
\text { their personal } \\
\text { memories to save } \\
\text { information they } \\
\text { collected from the } \\
\text { Internet }\end{array}$ & $\begin{array}{l}\text { Students } \\
\text { communicated } \\
\text { through the devices } \\
\text { to give and receive } \\
\text { feedback on their } \\
\text { saved personal } \\
\text { work }\end{array}$ & $\begin{array}{l}\text { Students saved } \\
\text { and retrieved } \\
\text { their personal } \\
\text { digital } \\
\text { products }\end{array}$ & $\begin{array}{l}\text { External } \\
\text { personal } \\
\text { memory }\end{array}$ & \\
\hline $\begin{array}{l}\text { Collective } \\
\text { memory } \\
\text { coordinator }\end{array}$ & $\begin{array}{l}\text { Students had } \\
\text { collective } \\
\text { memories } \\
\text { including } \\
\text { information about } \\
\text { class activities } \\
\end{array}$ & $\begin{array}{l}\text { Students } \\
\text { communicated } \\
\text { through the devices } \\
\text { to create their } \\
\text { collective memories }\end{array}$ & $\begin{array}{l}\text { Students saved } \\
\text { and retrieved } \\
\text { their collective } \\
\text { digital } \\
\text { products }\end{array}$ & $\begin{array}{l}\text { No } \\
\text { evidence } \\
\text { found in } \\
\text { the study }\end{array}$ & $\begin{array}{c}\text { Collective } \\
\text { memory } \\
\text { coordinator }\end{array}$ \\
\hline $\begin{array}{l}\text { Trial-and-error } \\
\text { learning space }\end{array}$ & $\begin{array}{l}\text { No evidence } \\
\text { found in the study }\end{array}$ & $\begin{array}{l}\text { Students } \\
\text { communicated } \\
\text { about their digital } \\
\text { experiences and } \\
\text { trials }\end{array}$ & $\begin{array}{l}\text { Students } \\
\text { practised trial- } \\
\text { and-error } \\
\text { learning to } \\
\text { create their } \\
\text { products }\end{array}$ & $\begin{array}{l}\text { No } \\
\text { evidence } \\
\text { found in } \\
\text { the study }\end{array}$ & $\begin{array}{l}\text { No evidence } \\
\text { found in the } \\
\text { study }\end{array}$ \\
\hline
\end{tabular}

\section{General Factors that Shaped All Ways of Use}

The education system in New Zealand shapes using devices in classrooms by allowing them to be part of the educational tools in classrooms. Kathy valued the flexibility the curriculum gave to her as a teacher: "It's ... not telling you exactly what to teach and how to teach, it's actually giving you a scope ... heading in a direction that you and the kids take it". All uses 
were enabled due to the flexibility of the curriculum that allowed Kathy to choose and design the learning activities, the teaching strategies, and the resources used including digital technologies. At the same time, the National Curriculum provided a framework that clarified desirable competencies for learners, called 'key competencies'(Ministry of Education, 2007). Kathy reported, "the curriculum ... I think it's really awesome and ... it's very clear about the place of those competencies, it's driven by really important principles". After the final interview, she wrote in an email how they influenced the school vision:

I forgot to mention ... how hugely influenced we are by the key competencies in the NZ Curriculum. (Relating to Others, Managing Self, Thinking, Participating and Contributing and Making Meaning from Language Symbols and Texts). We believe that it is essential that our students are able to use these competencies as 'learning power' and that the competencies will enable them to be confident, capable life-long learners who 'shine' in a range of contexts.

Kathy described how the key competencies influenced her teaching: "So this is what we are always thinking about when we plan, facilitate, and reflect on our learning. Our kids are able to talk about their learning using these key competencies". She employed the key competencies in her pedagogical practices when using digital devices, as will be described in the contribution of digital devices.

The other general factors were related to the school system. The decision of integrating digital devices into School A was taken by the school leadership, Kathy said:

I guess it was really driven by [the principal] and using her understanding of social justice. [The principal] was very keen that all of our students would have access to digital tools to enable there to be equitable outcomes for them. I guess [the principal] was very keen that our kids were not 'left behind' because they didn't have access to devices. So [the principal] worked with others to set up a trust ... to provide families with a way to access devices.

The school staff also contributed to integrating devices in teaching and learning. Kathy said, "There were others in the staff, of course, who knew the benefits of the children using digital devices and we were really keen". The school communication with the families was important from Kathy's perspective: "I think one of our huge drivers was also home and family communication and just having the parents much more aware of what the kids were 
learning because they use the devices. So that was really big". The decision of the integration, which had support from the staff and families, was followed by practical steps.

School policies shaped using devices such as the BYOD policy and 'Kawa of care' agreement, which was one of the tools used to guide how students should use digital devices in the school. A trust was set up to offer reasonable deals for purchasing Chromebooks with insurance, modems, and data to facilitate this for families facing economic difficulties. Kathy clarified:

The Chromebooks were all purchased by families from ... a trust set up to provide ... school students with Chromebooks to enhance their learning. They can pay off the Chromebook in small weekly instalments. Only some can take them home if they and their families have 'passed' the Kawa of Care.

Kathy reported that only two students in her class did not have their own digital devices and the school estimated that $90 \%$ of its students from Years 4 to 8 were using digital devices for their learning. These policies influenced students' use, as students' commitment to the 'Kawa of care' was observed and reported by Kathy. Classroom designs enabled charging, using, and keeping devices in safe spaces. The school provided stable and fast Internet access for the students and staff. The school offered technical support for students' devices where applicable. Kathy reported, "We used to have technical issues but we just don't seem to anymore". Mark supported that and said that sometimes the Internet at school became so slow but "It's not really happening much".

Another practical step was preparing teachers to use digital technologies in their teaching, such as providing SAMR workshop for the teachers in the school. Infographics about this model were hung in the staff room and documents related to it were part of the school curriculum. This was one of the factors that influenced Kathy.

The general factors relating to Kathy included her beliefs, education, theoretical perspectives, pedagogical knowledge and practices, and digital skills. She believed that learning is "finding within yourself the knowledge, understandings and skills and ways of being the best that you can be". Learning happens in her opinion "when something is drawn out from you, and there's an exchange of ideas and skills". She said that learning needs a context to happen: "I think it's the facilitation, that's the real key. It's setting up an environment for people to grow and develop together". For this reason, she thought that children learn best "with each other" and "with high expectations and with a feeling that they 
are valued, and that they are safe, and that they are respected and for who they are and for what they bring to that learning community".

Kathy's perspectives about learning were influenced by different factors, which included pre-service and in-service teacher education. She developed her critical point of view about social justice in education while she was studying at Teachers' College. Following this, some initiatives transformed her ways of teaching. The first was about how students could create success criteria to self-assess their learning against the key competencies of the New Zealand Curriculum. The others included culturally responsive teaching, and developing inquiry communities of mathematics, problem-solving, and group working.

Kathy considered that her strengths as a teacher lay in language and arts, not in technologies, and said, "I'm a special needs IT person in the school", meaning she felt she needed extra help. Nonetheless, that did not stop her from integrating digital technologies in her teaching. To overcome this issue, she participated in teachers' professional workshops about integrating digital technology in teaching. She added that she learnt through collaborating with digitally skilled teachers in her school. One of the participant students, Peach, commented on Kathy's efforts to enhance her digital skills:

She $[$ Kathy] is really helpful because she goes out and does courses about these kinds of things ... Because for people like her, she's been using paper and stuff in her days when she was little, and now she's learning new skills.

She added that teacher's learning of digital skills meant that they were also learning. This indicates that Kathy was open with her students about her struggles in using digital technologies. In return, her students appreciated her efforts to enhance her digital skills..

In general, Kathy believed that the device "always, is a tool. It's only ever as a tool to make the learning transformational and enhances the learning ... It makes you better able to achieve your purpose for learning”. This belief underpinned her integration of the devices in her teaching beside her theoretical perspectives, which were influenced by the SAMR model. This model categorises four different degrees of classroom technology integration. She clarified that her approach:

...Is based on a model, SAMR model so if it's just a substitution, then it means you're just doing something that you could do with another tool. But if this is going to 
enhance your learning, it's going to make it different in much clearer for you and much more engaging for you. It's probably even more than engaging ... it's going to make you have deeper learning because of the tool.

As showed in the previous sections, students' digital skills and beliefs about digital devices shaped all the uses; as Kathy said, "They're [the students] very, very savvy at using the devices". These skills were developed as a result of many other factors at the same time as it influenced how the students used their devices.

All of the factors mentioned were influenced by the development of Web 2.0 technologies. These developments created countless opportunities that enabled all ways of use. When these technologies were disrupted, all the uses were paralysed. On some occasions, the participants stopped using their devices when they lost Internet connections, for example. In the interviews, the participants and Kathy agreed that Internet problems significantly affect using the devices.

\section{Summary}

In this case, using digital devices was classified into six ways of use: a source of information, a means of communication, a trial-and-error learning space, a production medium, an external personal memory, and a collective memories creator. These ways of use did not work in isolation; they triggered or were triggered by each other. The findings showed that synergistic factors related to different elements that enabled and shaped the uses; no single factor could be claimed to solely cause or influence how these ways of use happen or were shaped. The five elements that interacted in classroom system were (1) factors related to the teacher; (2) factors related to the digital technologies; (3) factors related to the students; (4) factors related to the school and classroom environment; and (5) factors related to the education system of New Zealand. There were general factors that influenced all uses and specific factors that shaped particular uses.

\section{The Contribution of Uses to Educational Purposes}

This section reports how using digital devices contributed to the desirable educational purposes in Kathy's class. The objectives of the learning activities were classified based on Biesta's (2009) framework of educational purposes adopted in this study. In Kathy's classroom, learning activities were framed around the five key competencies of the New Zealand National Curriculum (Ministry of Education, 2007). The curriculum considered the five competencies essential for students learning in all learning areas and, therefore, "The 
development of the competencies is both an end in itself (a goal) and the means by which other ends are achieved" (Ministry of Education, 2007, p. 12). Kathy and the students used that framework to evaluate their learning in almost all learning activities including the observed learning activities: the STEAM workshops and literacy activities. The five key competencies were thinking, using language, symbols, and texts, managing self, relating to others, and participating and contributing.

\section{Qualifications}

Qualification functions mean equipping learners with the knowledge and skills that enable them to do specific or general tasks or jobs. Using language, symbols, and texts is the competency that meets this function, as shown in the next section.

\section{Using Language, Symbols, and Texts}

This competency was defined in the New Zealand curriculum as:

Using language, symbols, and texts is about working with and making meaning of the codes in which knowledge is expressed. Languages and symbols are systems for representing and communicating information, experiences, and ideas. People use languages and symbols to produce texts of all kinds: written, oral/aural, and visual; informative and imaginative; informal and formal; mathematical, scientific, and technological. Students who are competent users of language, symbols, and texts can interpret and use words, number, images, movement, metaphor, and technologies in a range of contexts. They recognise how choices of language, symbol, or text affect people's understanding and the ways in which they respond to communications. They confidently use ICT (including, where appropriate, assistive technologies) to access and provide information and to communicate with others (Ministry of Education, 2007, p. 12).

Using the devices as a source of information increased students capabilities to access and deal with different types of information, as shown. The devices enabled the participants to manifest their learning in different ways and engage language, symbols, text, and sounds by producing different digital artefacts such as 3D face models, the logo, stories, blogs, and their evaluation of the literacy. They were able to use different features in applications such as fonts, colours, spell-check, and paragraph settings to create their work. For example, as in Figure 4.14, below, Mark used his device to create a learning story that included images, mathematical symbols, and words; Sam used his device to write a story and used images and 
dialogue format; Peach used her device to create an assignment and used numbered and bulleted lists and paragraphs. Being able to interpret and use words, numbers, images, movement, metaphor, and technologies required trial-and-error learning. Mark and Sam spent time doing their trials before creating 3D models, and Peach before creating her logo and other digital products.

\section{Figure 4.14}

Participants Using Language, Symbols and Text

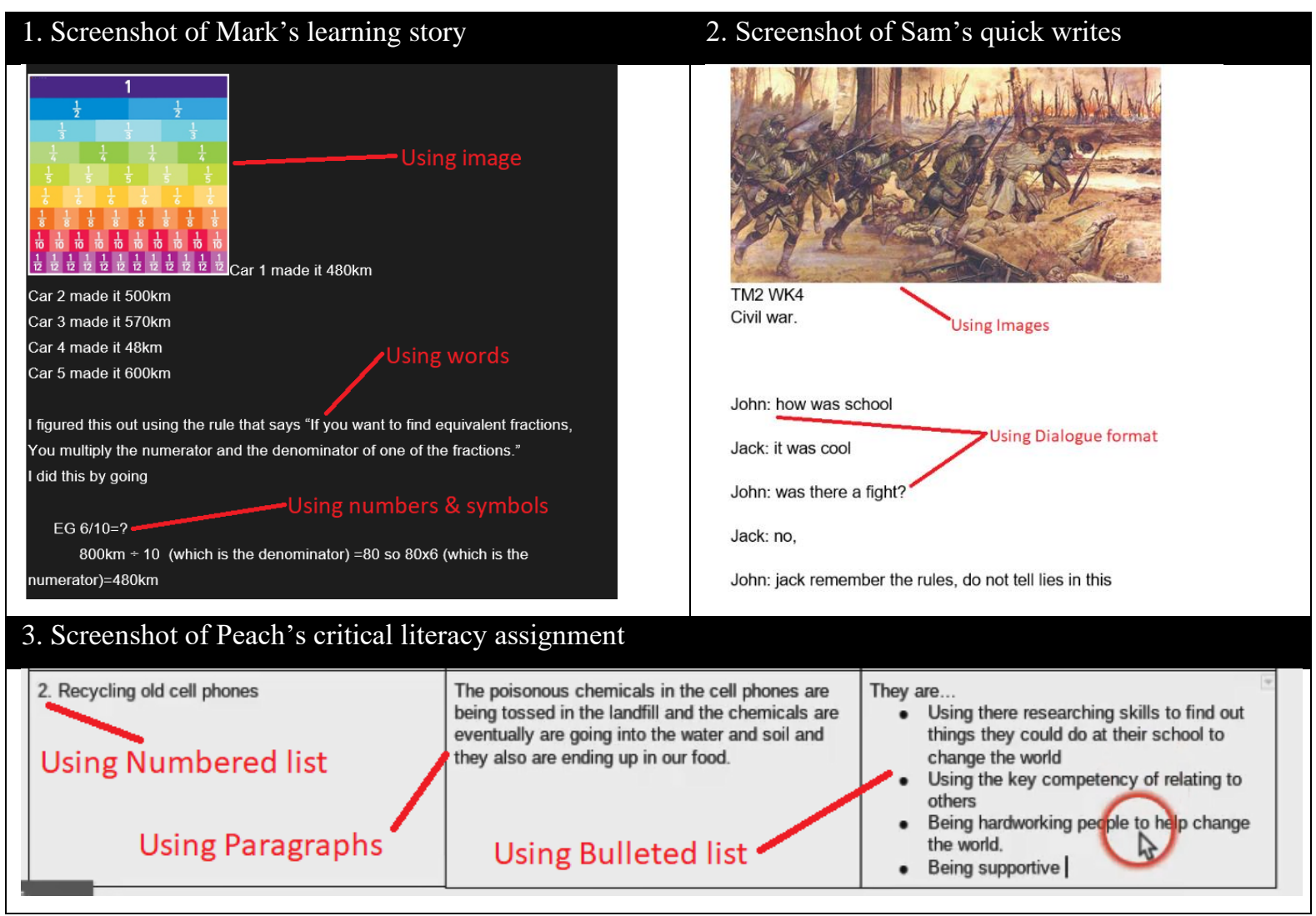

These productions were created individually or collaboratively, that is, personal or collective memories. Then they were shared with teachers, peers, and family members to communicate their ideas and receive feedback through the devices, that is, means of communication. This all contributed to their knowledge and skills. The students also used and communicated about devices, which influenced their digital skills. Kathy said:

Through that device, it's the communication of their thinking and ideas with other people, other experts or with a family or with me or so. And that's what makes it really, you know, really worthwhile for them to be using it. 
This influence on students skills related to using language and texts was not always desirable. For handwriting, Mark critically talked about the influence of using digital devices on his handwriting. He said: "I'd rather write because my handwriting is really bad"; he added, "sometimes they [digital devices] can be helpful for writing, but for me not really". From Mark's point of view, depending on typing most of the time did not give him much time to practise handwriting and enhance it.

Another controversial influence was related to using the spell check function. Sam and Peach said, during the interview, that digital devices helped them in spelling. This help, as observed, was to correct the words directly while typing. This use enabled the students to create texts without spelling errors, but no evidence found that it helped them to learn the right spelling of the corrected words; especially during the observations both participants regularly misspelt words while writing.

\section{Socialisation}

The socialisation function is related to being an effective member of the community. Two competencies align with this function: relating to others and participating and contributing.

\section{Relating to Others}

This competency means:

Relating to others is about interacting effectively with a diverse range of people in a variety of contexts. This competency includes the ability to listen actively, recognise different points of view, negotiate, and share ideas.

Students who relate well to others are open to new learning and able to take different roles in different situations. They are aware of how their words and actions affect others. They know when it is appropriate to compete and when it is appropriate to cooperate. By working effectively together, they can come up with new approaches, ideas, and ways of thinking (Ministry of Education, 2007, p. 12).

Using devices to create collective memories such as critical literacy assignments contributed to students' collaboration and sharing ideas, as shown in the previous sections. Kathy said, "It's when ... some of those apps are really getting them to develop those social skills, and talk and challenge each other and, you know, make connections at a deeper level".

Using the devices as a means of communication facilitated sharing ideas and working together among the students. Kathy mentioned that her students learn effectively 
when they communicate with each other and share their digital skills: "They [the students] can do things for each other ... once they do something, they can just find it and do it really easily, and they can find really awesome ways of doing it." This was consistent with what Mark said in the group interview: "Chromebooks are quite easy to use because it's like once you learn how to use it, you can teach others as well". Sam said he liked asking his friends how to do new things, but he preferred asking his teacher first. Peach agreed with them that she asked her friends at school and her family at home about using digital technology. That shows that communication with others about using the devices influenced how students relate to each other.

The participants communicated through the devices basically to get feedback and share ideas about their work. Figure 4.15, below, shows an example of how communicating through the devices enabled sharing ideas and recognising different points of views. Sam received feedback from Kathy about his writing and asked for feedback from Mark.

\section{Figure 4.15}

Sam Receiving and Asking for Feedback

\section{Kathy's feedback to Sam}

Once upon a time was a man and his name was peter and peter was a soldier and he had everything he ever had, like his 3 brothers and their names are james, jack and john look out for each other even though when hard moment's. When my brothers were scouting they found dead body's in the south and it was our soldiers that were guarding our walls, then they came back to report about

\section{Sam asking for Mark’s feedback}

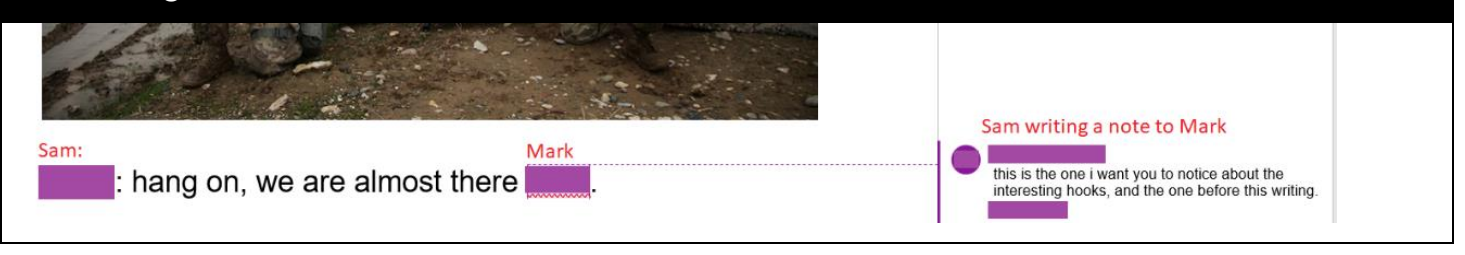

Another example in Figure 4.16, below, shows how both a teacher and a student provided feedback on Mark's blog. The feedback was positive and encouraging, as rules for giving feedback in the classroom required being aware of how words affect others. 


\section{Figure 4.16}

\section{A Teacher's and a Student's Feedback on Mark's Blog}

\section{Teacher's and peer's feedback on Mark's learning story posted on his blog}
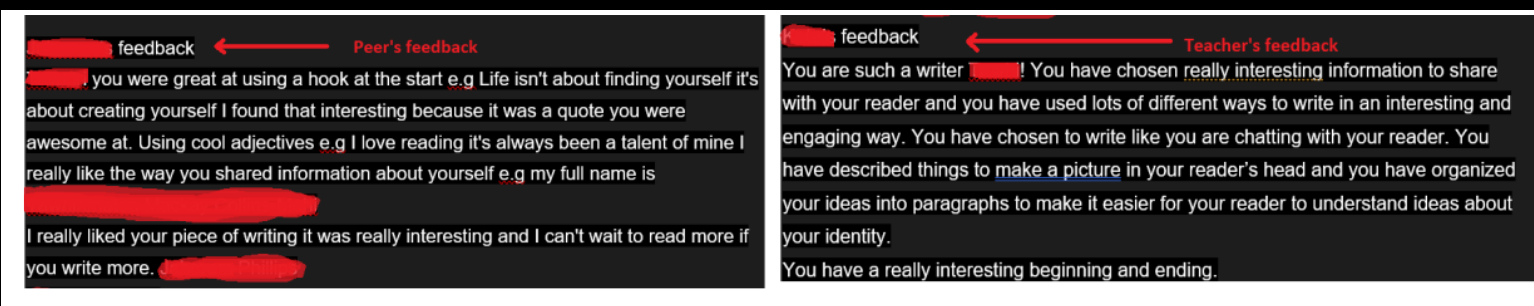

\section{Participating and Contributing}

The definition of this competency, which aligns with the socialisation function, was:

This competency is about being actively involved in communities. Communities include family, whānau, and school and those based, for example, on a common interest or culture. They may be drawn together for purposes such as learning, work, celebration, or recreation. They may be local, national, or global. This competency includes a capacity to contribute appropriately as a group member, to make connections with others, and to create opportunities for others in the group.

Students who participate and contribute in communities have a sense of belonging and the confidence to participate within new contexts. They understand the importance of balancing rights, roles, and responsibilities and of contributing to the quality and sustainability of social, cultural, physical, and economic environments (Ministry of Education, 2007, p. 13).

Kathy considered her classroom to be a community of learners and that all its members should contribute by sharing their 'golds'. The 'gold' was a metaphor used regularly in Kathy's classroom to refer to the knowledge, skills, and expertise of someone. The students should look for the 'gold' in others and share their 'own gold' with others. In this class, both communicating through and about the devices allowed the students to contribute as group members and make connections. That was observed when the students shared their expertise in using their devices and when they actively gave their feedback through them.

Additionally, the students collaboratively participated in creating and accessing collective memories and digital products, such as their literacy assignments, where they could contribute to and benefit each other. Observing others' online behaviours changed 
Peach's reaction. At the beginning of creating the Google Logo shared document, Peach said that she could only write one sentence as she could not think of any other idea. She told me that she didn't have the confidence to start typing her ideas; she even moved her section, using copy and paste, to the last part of the document. After reading her group's contributions, she started to develop more sentences and ideas and ended up with a paragraph, as in Figure 4.17, below. The process of creating a collective memory, communicating through the device, and reading others' contributions encouraged Peach to come up with new ideas and share them with others.

\section{Figure 4.17}

\section{Peach Writing with her Group on the Same Document}

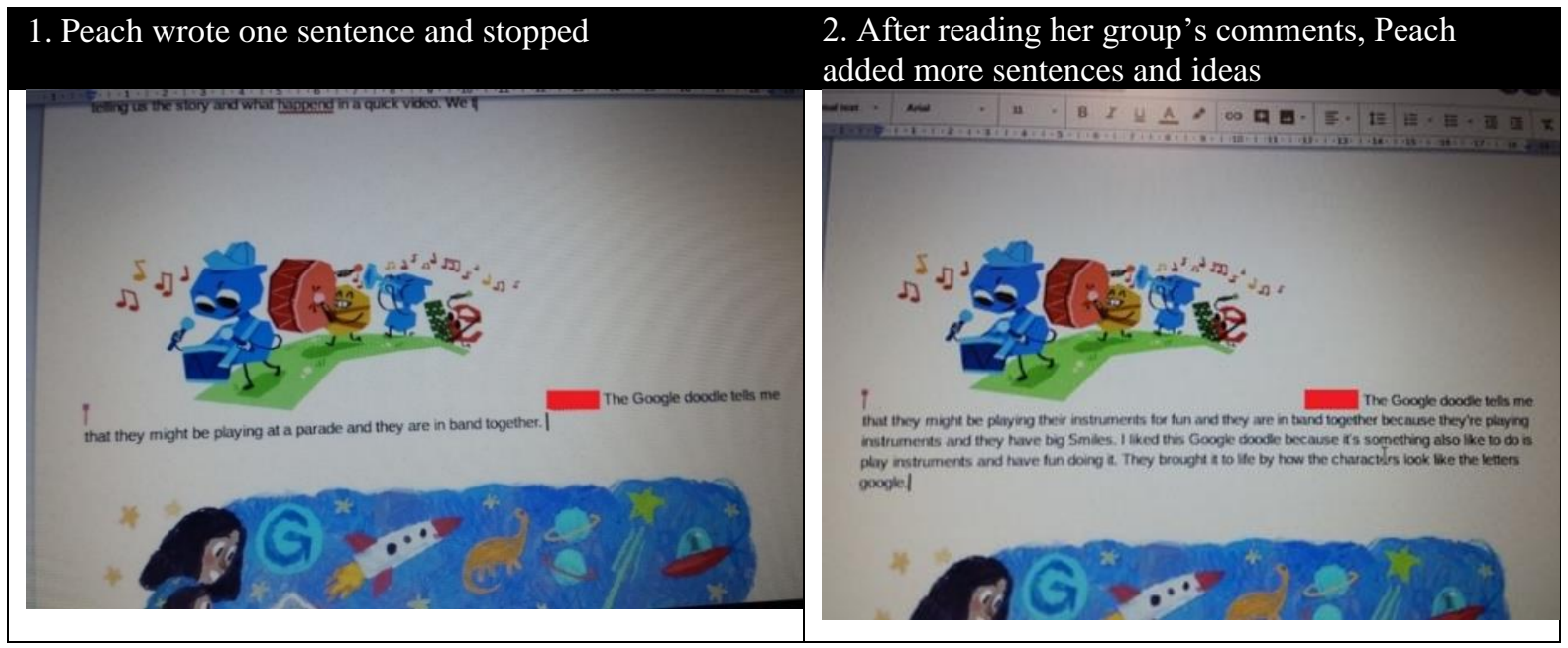

Kathy reported that students who did not have digital devices faced difficulties:

Not having a digital device can affect collaborative problem solving I think, it's actually really important that they can access that, I just have two children who don't have Chromebooks, but they are able to access an iMac, and it's quite frustrating for them if they can't access a device, especially around solving a problem collaboratively, you know, they need to actually be with other people with Chromebooks.

Kathy clarified that that does not apply to all learning areas:

I guess that the balance will be to ... the Chromebook is actually no help when you're doing your collaborative problem solving, for Maths, it's not helpful at all in that sort of setting because you are solving this problem, it's on a piece of paper, and you are coming up with your group strategies. 
Therefore, while devices can play a role in participating and contributing, there are some educational activities that can run effectively without them.

\section{Subjectification}

Two competencies aligned with the subjectification function: thinking and managing self. Subjectification is about reinforcing independent thinking.

\section{Thinking}

Thinking was defined in the New Zealand national curriculum as follows:

Thinking is about using creative, critical, and metacognitive processes to make sense of information, experiences, and ideas. These processes can be applied to purposes such as developing understanding, making decisions, shaping actions, or constructing knowledge. Intellectual curiosity is at the heart of this competency. Students who are competent thinkers and problem-solvers actively seek, use, and create knowledge. They reflect on their own learning, draw on personal knowledge and intuitions, ask questions, and challenge the basis of assumptions and perceptions (Ministry of Education, 2007, p. 12)

Kathy explained her approach, which reflected this goal:

All workshops and other learning tasks in all curriculum areas involve solving a problem or answering a wondering question in groups. The students develop success criteria before, during and after the lesson. We all reflect on these success criteria and make a connection to a big idea that we've learnt.

The students reported that using the devices as a source of information increased their knowledge and played a role in their decision making. Mark said in the interview that using digital devices "helps me to, like, have more information", which Peach and Sam agreed with. Mark searched the Internet about the key competencies framework to evaluate the literacy assignments. Sam searched YouTube to listen to music to understand how music changes our emotions. He said that he wanted to listen to "heaps of artists" and justified, "because we are trying to learn what's going on these songs". He stopped on a song and tried to analyse what feelings the song created: "this song is about how they [the actors in the video] felt when they have friends". He added, "they have feelings of friends, and they wanted to share with other people how they felt, and if they had the same feelings as them". By listening to different songs, Sam was able to recognise a specific emotion that music can 
create; he then created his own piece of music that created other feelings. He wrote on his blog commenting on the Music workshop, "I was good at thinking, so thinking about what kind of music should be doing". In the Google logo workshop, Peach was curious about the meaning of 'Lunar'; she used the devices to search and learn the meaning. However, it was interesting when Mark, in an incident, chose not to use the devices to get the information he needed as he considered the devices as an option but not the only source.

Using devices as an external personal memory contributed to participants' thinking and making decisions. Peach said that the mind map she used helped her to "get ideas" about herself to design the logo. She picked three elements: her interest in music, in netball, and in the arts (Figure 4.18, below). She did that too when she wanted to do her critical literacy activity; she opened a saved document from her drive and said "we wanna get ideas" about how to evaluate the literacy piece.

\section{Figure 4.18}

\section{Peach Using the Devices for Thinking}

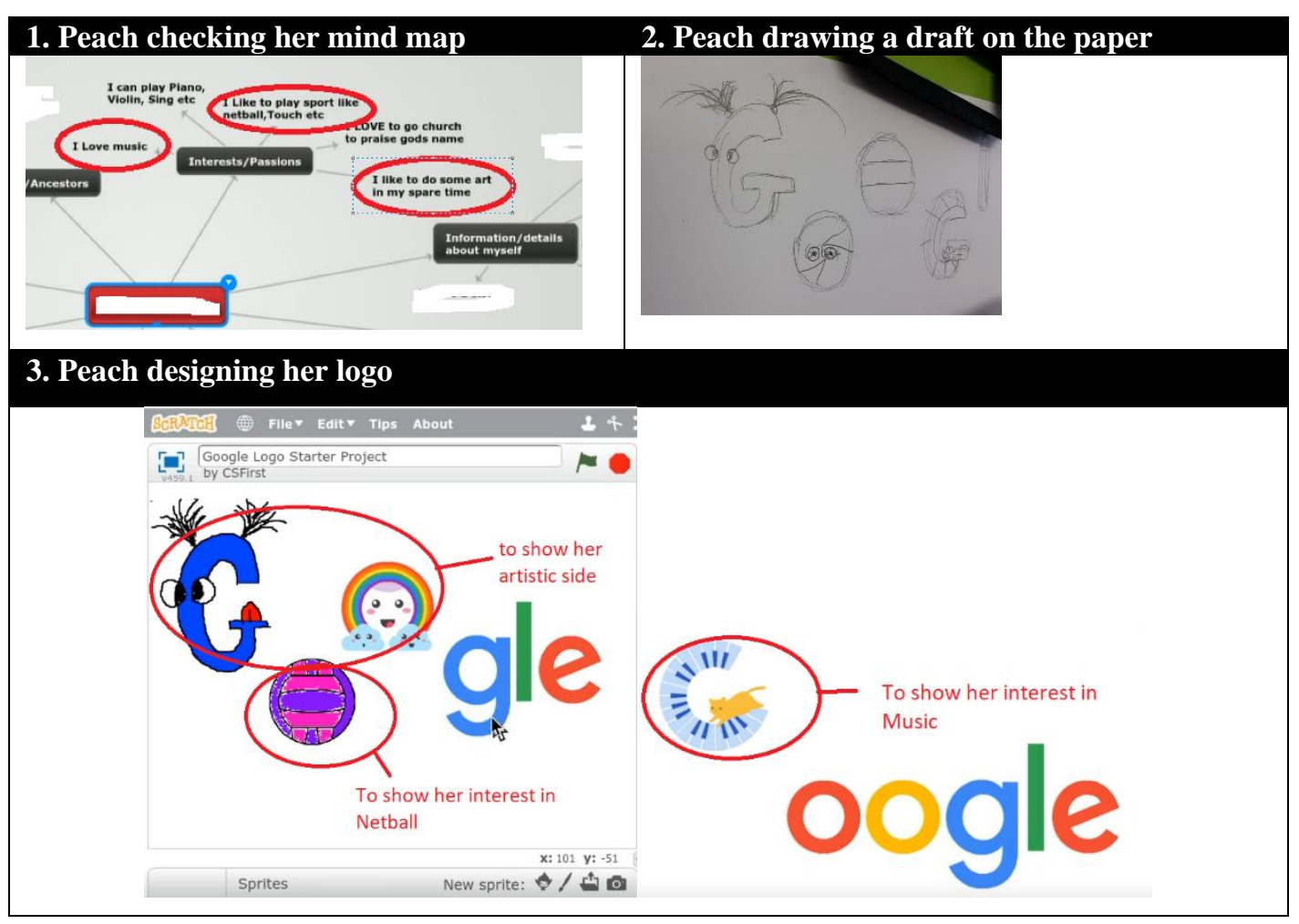

With regard to problem-solving, Kathy said, "in all learning areas we have a problem to solve" and using the devices could be helpful. Problem-solving involves seeking, using, and creating knowledge. The devices facilitated these three aspects. Kathy gave the following example: 
Last year, for example, there was a problem ... we had some bird boxes, and the Tūî weren't using them. So we had to try and find out some information about the Tūî which would let us understand more about why they weren't using the bird boxes, so ... it was a little bit of research. But then actually when it came to designing the boxes it was ... an app where you create it. And so the problem was sort of, I guess, [a] technology problem or a project to meet a need. So the digital devices were partly for research, but they were also using an app where you were creating something.

This quote addresses several uses that influenced solving the problem: using the devices as a source of information about the bird, and using the device as a production medium to design the bird box. Consequently, in this case, using digital devices as an external personal memory, source of information, and production medium contributed to students' thinking. The contribution included developing understanding, making decisions, shaping actions, problem-solving, and increasing their knowledge.

\section{Managing Self}

This competency was defined as:

This competency is associated with self-motivation, a "can-do" attitude, and with students seeing themselves as capable learners. It is integral to self-assessment. Students who manage themselves are enterprising, resourceful, reliable, and resilient.

They establish personal goals, make plans, manage projects, and set high standards. They have strategies for meeting challenges. They know when to lead, when to follow, and when and how to act independently (Ministry of Education, 2007, p. 12).

Using the devices as a trial-and-error learning space and production medium influenced this competency. On the whiteboard of the classroom, the students wrote the following sentences to reflect on their learning after using the devices to design the 3D Modelling images such as, "investigation what the app can do", "took a risk", "testing", "not giving up", "practising", "mucking around", "trying new things", "does not have to be perfect" (see Figure 4.19, below).

These sentences followed using the devices in the implementation phase. Sam wrote on his learning story about the Music workshop, "The hard thing for me was managing self because sometimes I take risks and the music sometimes goes wrong and that is what my next steps are". While working, Mark mentioned that he was trying new things such as new functions and features. Peach, who modified her logo three times, commented on that 
iterative process: "I have learned that designing is hard, I had to say". To produce digital artefacts, the students had to try new things, challenge difficulties, and explore new learning areas. That was part of using the devices as a trial-and-error learning space, which contributed to their self-management.

\section{Figure 4.19}

Students' Reflections on their Learning

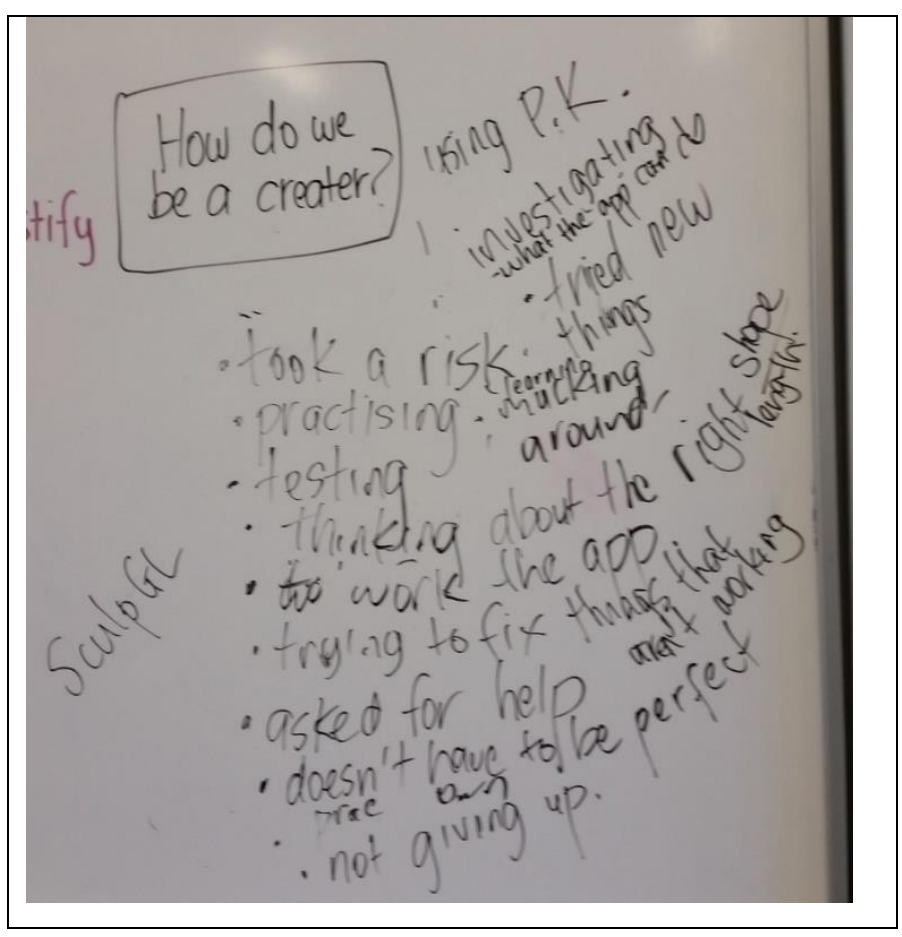

\section{Summary}

The six ways of use contributed to the objectives of social actors' educational purposes, which was framed around the five key competencies of the National Curriculum of New Zealand. The five competencies are thinking, using language, symbols, and texts, managing self, relating to others, and participating and contributing. The six ways of use contributed to qualification functions. Production medium, means of communication, and collective memory uses contributed to socialisation functions. Using the devices as a source of information, production medium, trial-and-error learning, and personal memory contributed to subjectification. The contributions of the devices resulted from the interactions that happened between the factors related to the five elements in classrooms. 


\section{Chapter 5 Case Study Two}

This chapter reports the findings of the second case study. Following the organisation of the previous chapter, I first describe the context of School B, the classroom, the participants, and a general description of the observed learning activities. Then I report the findings related to the ways of use and the factors that shaped them. Finally, I report the contribution of the ways of use to the educational functions.

\section{The Context: The School and The Classroom}

School B was a full primary school from Year 1 to Year 8 with a roll of 210 students. The students came from different backgrounds, creating a multicultural community which included 42\% Pākehā students, 13\% Māori, and 10\% Pacific heritage students. There were also English Language Learners from Asia, the Middle East, and Africa. Because of this widely diverse community, one of the school's main values was improving students' wellbeing and valuing cultural diversity.

Each class in the school contained 25-28 students from two consecutive year levels. The classroom spaces in the school were separate, which means they were not designed as an Innovative Learning Environment (ILE). Each classroom space contained a projector, whiteboards, an artwork corner, a corner for the digital devices, a small library, and movable tables and chairs for collaborative learning. The teachers of the same year levels worked collaboratively; they planned the programme together and often mixed combinations of students between two classes. For example, the classroom that I observed combined Year 7 and 8 students. It was usually mixed, with another class of the same year levels forming a cohort of 55 students learning together in two learning spaces. Besides the cultural diversity in the classroom, there were students of different learning capabilities and different social and behavioural characteristics.

The next section introduces the teacher Tess and the four students, Bob, Mitchel, Alice, and Ian, who participated in this study.

\section{The Participants: A Teacher and Four Students}

I first met Tess when she volunteered to participate in the pilot study for this research project. She was interested in the research topic and agreed to participate in the study. Tess was teaching in a classroom combining Year 7 and 8 students. I discussed with her which students 
could be invited to participate, as I was looking for four students with different learning strengths and different levels of digital skills. Tess suggested eight students, and the first four students agreed to participate in the study: two from Year 7 and another two from Year 8.

Tess

Tess was a primary teacher with diverse teaching experience. She had completed a three-year diploma course for teacher training in New Zealand. After graduating in 1984, she taught in New Zealand for two years before moving to the United Kingdom where she taught for more than 10 years. She returned to New Zealand in 2000 and started working in School B nine years ago, teaching Years 5 and 6. In the last four years, she had been teaching children in Years 7 and 8 and had a leadership role in her school. She described her classroom as having a "really fun, happy, nice, interesting bunch of kids". Her motivation to participate in this study was:

Are there kids who are much more capable and knowledgeable about what the devices can do than I am? I know that there are kids like that and are sitting there thinking, well, really it would be much better if we did this kind of work or if we use them in this kind of way ... I'd be fascinated to know. Do they have the secret knowledge that [they are] not sharing with me and they don't use?

\section{Bob}

Bob was in Year 7. Tess described her as a "motivated" and "enthusiastic" learner. Tess added, "she's so keen to learn and full of ideas. She's got a very creative kind of brain. She also just understands things very quickly, so she only needs something explained to her once". This was supported by my observations. For example, Bob quickly understood how to use Screencastify for recording with the think-aloud interview, even without the prompt questions. Tess mentioned that Bob's creative side affected her achievement "if things are too prescriptive, too rigid, she just doesn't meet the criteria because she does what she wanted to do anyway". For example, Bob might fail a formal assessment because "she might choose not to meet the criteria ... Because she would think that she had a better idea".

While working on her device, Bob showed commitment and concentration while doing her tasks. She regularly urged her group members not to be distracted by the Internet and focus on the work. She used the devices confidently and was open to learning new things. 


\section{Mitchel}

Mitchel was in Year 7. He said, “I don't really like school”. He justified, “I don't think I'm ever going to like school because I'm not very smart. And you have to be really smart to like school. And I'm not very good at subjects. I'm not good at Science, Maths, English”. He thought that he was good at art and when he grows up, he would "become a musician or basketball player or skater".

Tess had a different point of view about Mitchel's potential: "he is actually very clever. He has a lot of good ideas. He has a lot of knowledge". In her opinion, the reason for his attitude was that "he has no confidence in his own ability to read and write ... he's very negative about his ability to read and write and anything that involves anything that even looks like reading and writing or Maths". To help him with his learning, he usually had a teacher aid who provided support to him while working on his tasks.

When using the devices, Mitchel was more interested in using them for gaming rather than for academic purposes. He was easily distracted when using the devices. He avoided tasks that required typing or reading and preferred using videos for his learning. Tess was aware of that. Tess mentioned that Mitchel learnt better by watching, doing, and talking, not reading. Mitchel supported that when he reported that he preferred learning by watching, which explained his preference for arts and videos. To help him in writing, Tess said that she "just started trying with him using the voice typing on Google docs. So instead of typing, you just speak ... and he was very positive about that". Mitchel agreed that he liked this approach.

\section{Alice}

Alice was in Year 8. She participated in all of the data collection processes except for the group interview, as she was absent. Tess reported that "she was dyslexic, so the reading and writing could be a problem for her, but it isn't because she's got positive self-esteem ... and her strengths are really celebrated at home". Tess explained further: "the things that would support her differently would be her parents because she also talks a lot at home with her family about all of her learning".

Alice was competent when using the Internet and worked carefully on her digital documents. That was supported by her teacher, who said that she had good skills in using digital technologies, in general. 


\section{Ian}

Ian was in Year 8. He showed interest in science, especially physics. Tess praised his skills in reading, research, writing, maths, and science. She said, "he's already got a lot of knowledge that I think would have really supported him a lot and being able to be successful". She added:

He likes to have a lot of choices and be given a lot of control over what he is learning, how he's learning, how he presents it ... That definitely adds to his success as a leader and puts him off if he doesn't get a choice.

Tess mentioned that he chose his team carefully to ensure that they would work hard on their tasks. That was observed as he worked in harmony with his group and they were able to complete their research project earlier than other groups. Tess attributed his high achievement at school to his family "because I think they talk a lot about all of the schoolwork". During the observations, he showed proficiency in using the devices. He typed and worked fast and was able to use different applications easily and smoothly.

Students' digital skills, preferences and interests, and attitudes towards learning and how those influenced their usage of digital devices will be discussed further in the factors sections.

\section{The Observed Learning Activities}

When I asked Tess to suggest learning activities that required using the devices regularly, she suggested the 'scientific research learning activity' and the 'leadership learning activity'. These two activities were expected to be held weekly, and all class members would participate. The next section provides a general description of the activities. Then I report how the digital devices were used during these learning activities.

\section{Scientific Research Learning Activity}

The first activity that I observed was the scientific research learning activity, which was an annual activity in School B for Year 7 and 8 students. That meant that the teachers had experience in leading it, and that Year 8 students were undertaking the activity for the second time. Tess mentioned that a significant change happened to this activity three years ago when a parent, who was a scientist, helped in developing this activity. Tess reported that the other main source used to develop this activity was "a website which is designed for primary school teachers and kids, which has specific examples of the kinds of science experiments that the kids are doing. So there are a few websites like that". 
Tess highlighted the influence of the New Zealand Curriculum: "So this year, we looked at the curriculum to have a look at how science was divided up into different strands". She said that when they presented and told the students about what the science actually covers, they referred to the importance of understanding the scientific process and to the five strands of science in the New Zealand curriculum, such as the material world. The curriculum aspects that Tess mentioned state that by learning, science students "learn that science involves particular processes and ways of developing and organising knowledge and that these continue to evolve" (Ministry of Education, 2007, p. 28).

As the students of Year 8 had already taken this activity in Year 7, Tess and the other teacher re-divided the two classrooms into two groups. There was a group of students who had already done this learning activity or had the capability to do it with little supervision; Ian, Alice, and Bob were in this group. There was another group of students who would probably face difficulty in doing it, so would need more support from the teacher; Mitchel was in this group.

Tess said that the reason for designing and choosing this activity was, "to stimulate their [the students] interest in science and to open their eyes to the range of ideas that are science". The overall goal was "for them [the students] to understand the scientific process and to know how to carry out a scientific experiment. That's the big goal this year". The teacher added, "having a bit more of an understanding of what science is, being aware of the wide range of topics and subjects and ideas that come under the label of science".

This activity was expected to take one term. It was held between 11:10 am-12:30 pm every week and in the odd weeks there was an extra time between 1:30-3 pm. In the first week of the term, the teachers introduced the science topic. The students worked in small groups that they had chosen. Over the next eight weeks, they were expected to complete their research and present their learning depending on their topic, Tess said:

Either they have done an experiment, then they'll go through the whole experimental process, and they'll collect all their data, and they'll write their conclusions and present all of that on a big display board. Or if they've done some research, then they're going to need to create some kind of a demonstration of what they've learned about, maybe with a model and then some writing to explain the model. 
Doing scientific research was in conjunction with understanding the scientific concepts that underlined the research process. The observations and the collected worksheets showed how Tess and the other the teacher did that.

In the first two weeks, the teachers planned about 10 different practical science activities to be done with the students inside the classroom. Tess said, "a couple of those will be experiments, and the others are just little activities". The goal of these introductory activities was to "help the students to identify what areas of science they're specifically interested in. And then they're going to choose whether they do a science experiment or whether they do a more research-based project".

During that phase, the students did not use their devices. They listened to the teacher demonstrating the scientific process combined with interactive group work. Tess and the other teacher used worksheets to help the students to think, reflect, and plan for their research. The students were required to use a logbook as a diary to record their thinking and their progress while doing their projects. They also developed mind maps about the topic they would work on.

After the introductory activities, Tess and the other teacher prepared step-by-step instructions that included details of each research step for the whole term. They provided tools to help the students to complete their tasks such as worksheets, checklists, reflective questions, and diagrams. Some of these tools were shared online, such as slideshows or documents with links. These tools addressed concepts related to the research process, such as research question, testable question, variables, fair testing, and hypothesis. Tess explained the importance of these detailed instructions to the practical work:

To find out what actually is a testable question and to learn what that means in a scientific context. So learning about variables and learning about fair testing and learning how to make a fair test by repeating the tests lots of times to allow for anomalies and all those sorts of things. And then to be able to collect data and to analyse and present and to draw conclusions from the data and to be able to come up with a final conclusion that says whether or not their hypothesis is supported.

Each of the participants was a member of a different group that explored a different topic. Bob's group of three students examined serving sizes. Their research question was "Does changing the amount of food present to someone affect how much they take? Does it 
affect how much food they think the serving size is?" They did an experiment and collected data related to their inquiry.

Mitchel's group, which included him and one other student, investigated the influence of computer gaming on reaction time. Mitchel's group hypothesis was "We think that gaming is going to have a positive effect on our reaction time". They did an experiment to prove their hypothesis.

Alice worked with another student on "the science of photography. So how photography works basically, which means we need to find out ... how long cameras were made ... what technology was used back then". She and her friend investigated the similarities between cameras and eyes and the most simple of cameras, the camera obscura. They designed a model of the camera obscura.

Ian's group was the biggest; it included Ian and three other students; all were interested in science. He said that his group members were "quite interested in physics" and "we're thinking [of] going somewhere along the lines of magnets. Then one of our group members mentioned maglev trains, and we thought that would be a very interesting topic that we didn't know much about and would enjoy". Ian said, "our research, maglev trains, and how they work and the science behind magnetism, kinetic friction, also, how they're used ... today". Their research question was, "What is the science behind maglev trains?" They also designed a model of the maglev trains and were able to finish their research early to participate in a science fair outside the school.

\section{The Leadership Activity}

The leadership activity was held once a week. Tess's classroom was combined with the other class. The students were divided into two groups based on their Year level, not their capabilities; that is, Year 8 students in one group and Year 7 students in the other. Tess said that there were two different learning goals for each year level. For Year 7, "the objective in that class is for them to learn some skills that they will need to use when they're in the Year 8 leadership teams". That included "being able to send appropriate emails, and then they went onto learning how to use Google forms".

In the year in which I conducted this research, Year 7 had a different focus in each term. Term one was for a fundraising activity; term two was for email writing; term three for learning how to create Google forms; and term four for activities related to Te Reo Māori. 
Bob and Mitchel were observed during this activity in term three while learning how to use Google forms and how to write emails.

For Year 8, each student was part of a leadership team. There were, for example, a music team, fundraising team, and enviro team. Tess said that the students were expected to use "the skills that they learned at Year 7 plus a whole load of other stuff to actually try and achieve some things that provide some leadership in the school".

Ian was a member of the enviro team and lunchtime activities team, while Alice was a member of the music team. Ian and Alice worked collaboratively with their teams to achieve their tasks. They were expected to plan and implement the specific type of activity in the school independently, which in most cases required using digital devices. Tess explained:

The Year 8 groups are each split up into different teams that have responsibilities for organising and leading things throughout the school, and they have pretty much identified what it is that they're going to be leading. And so they'll be actually working through organising different activities, and that's a variety of things.

Tess reported that the students were assessed based on their overall achievements by the end of the year. The other way was "when they are working on it, I'm all the time interacting with the different groups, checking in on what they're doing, watching, talking to them, asking them questions". I was able to observe the second way of assessment, which happened while the students were working in the classroom. Tess, as described, was actively moving between the groups and providing support to them.

The main challenge for this activity, in general, was that some teams were more popular than others and most of the students wanted to be enrolled in them. The main challenge that happened during term three, in which I was doing my research, was the time that was allocated for this activity. Tess said, “As far as the leadership goes, I feel like that's been really interrupted and impeded by lots of other things getting in the way at school, unfortunately". Her evaluation of the implementation of the activity was:

I feel like this has been the worst time for a long time in terms of the success of the leadership programme. And that they haven't really had a lot of time available to them to achieve anything very much, so I'm not very thrilled with the leadership programme at the moment. 


\section{Summary}

In this case, Mitchel and Bob were Year 7 students and observed while doing a leadership learning activity for Year 7. Alice and Ian were Year 8 and were observed doing a Year 8 leadership learning activity. The four participants were also observed conducting a scientific research learning activity; Mitchel was researching reaction time with relation to computer gaming; Bob was researching food serving sizes; Alice was researching the science behind cameras; and Ian was researching the science behind maglev trains. The students used Chromebooks provided by the school; usually every student in the class had access to one of the devices.

\section{Using Digital Devices During Learning Activities}

In the next sections, I report the six ways of use extracted from this case and the specific factors that shaped them, which includes using devices as a source of information; a means of communication; a production medium; a collective memory coordinator; an external personal memory; and a research tool. Then I report the connections between uses followed by the general factors that influenced them.

\section{A Source of Information}

Using the devices as a source of information was observed through the whole term in this case, particularly during the science activity. The learning activities required interactive use, as the students needed to get information from the Internet to complete their tasks as it was their main source of information. The students researched from the Internet in different ways for different purposes. Some of the links were given by teachers and others were found by the students. Bob listed different questions related to their topic of serving sizes on a Google document, for example; "who invented serving sizes? and what are serving sizes for?" Then she started searching the web to find answers by typing the questions on the Google search engine. She also used her search history to locate information. She located information from Wikipedia, websites, videos from YouTube, and an academic journal (Figure 5.1, below). Although the final presentation of her group did not include a bibliography of the sources they used, the observations showed that she got information from different sources and dealt with different formats such as text, videos, and images. Mitchel's main source of information was videos on YouTube. He was observed watching several videos about reaction time and gaming. He typed keywords such as "reaction time" on the YouTube search engine. He used the videos to prepare for the experiment of his research. 


\section{Figure 5.1}

Screenshot of Bob's Screen While Searching

\section{Bob used questions for searching}

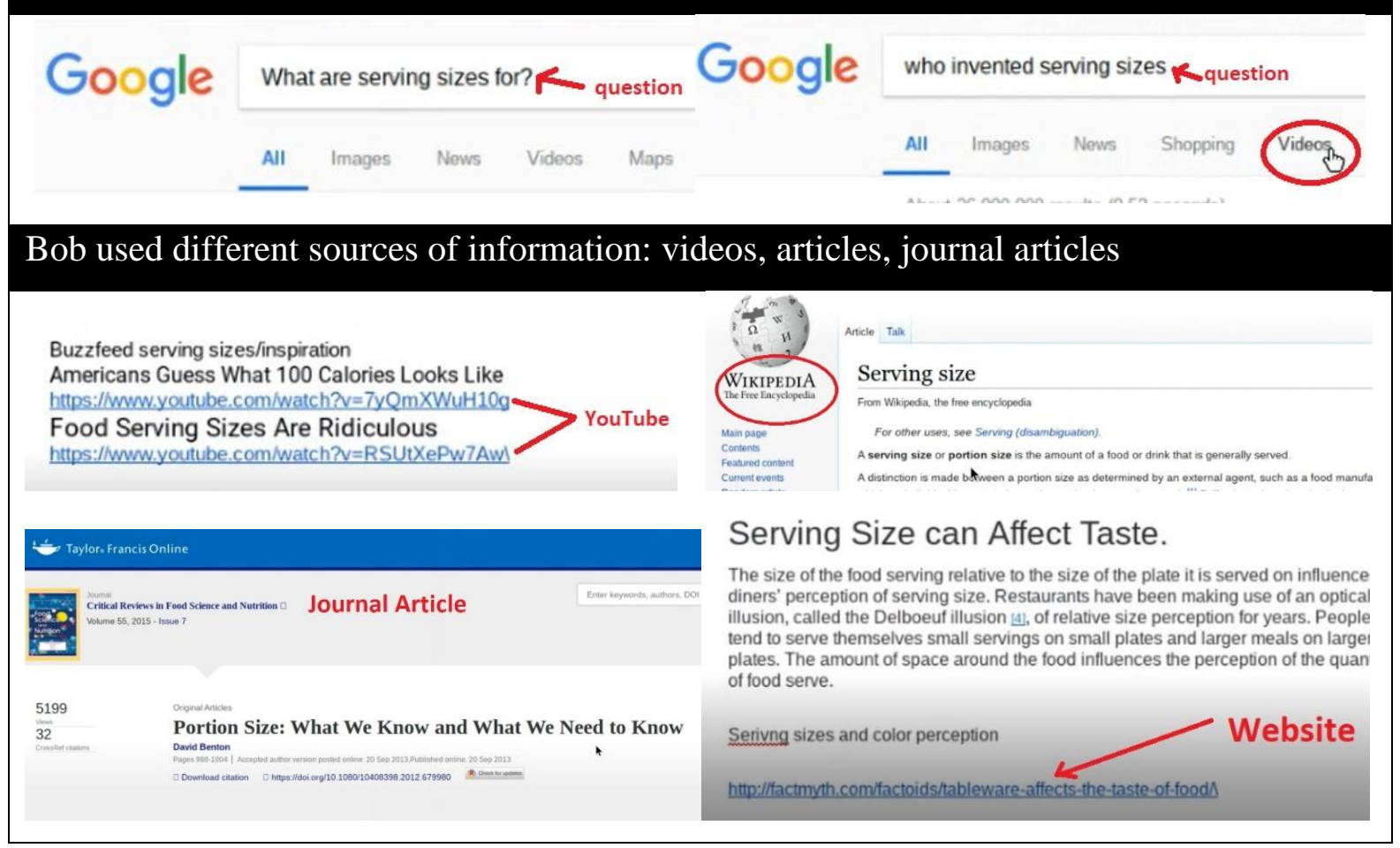

Alice used key terms to do her research on Google search. She included all the websites she used in her final presentation, which were all from educational or science websites (Figure 5.2, below). She expressed that she preferred to locate information from these websites as she did not trust any website. In the leadership activity, Alice reported that she searched the Internet to look for music and performers to run their music assemblies at school.

Ian typed key terms in the search engine to find out about friction, magnetism, and the origin of the maglev train (Figure 5.3, below). He used Google search to look for articles about these topics from Wikipedia, and educational websites. While reading the information he used the pop-up glossary definition for some words while reading in Wikipedia. Ian and his group also researched videos looking for different designs on YouTube to create their own model of a maglev train. They also searched the Internet to find magnets for their model. From the different websites that Ian and his friends researched and visited, they selected sources and listed them in their bibliography. The students not only used the search engines to look for information, but they also used documents, slides, and links sent from their 
teachers or peers. Bob and Mitchel were observed accessing these resources during the leadership activity.

\section{Figure 5.2}

Using Devices as a Source of Information by Alice

\section{Alice searched different websites to learn about light, lenses, cameras, and films}
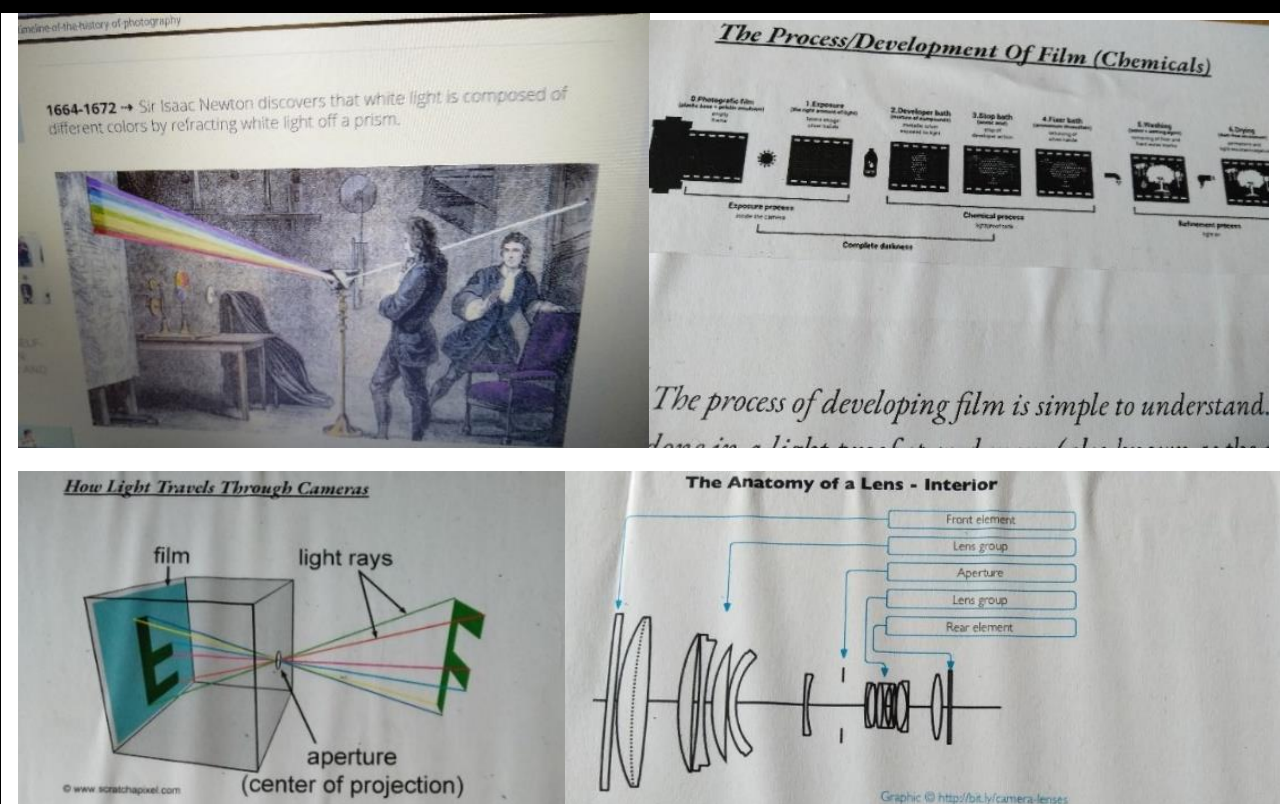

In a camera the word Lens is another word for bole. The sm

\section{Figure 5.3}

Using Devices as a Source of Information by Ian

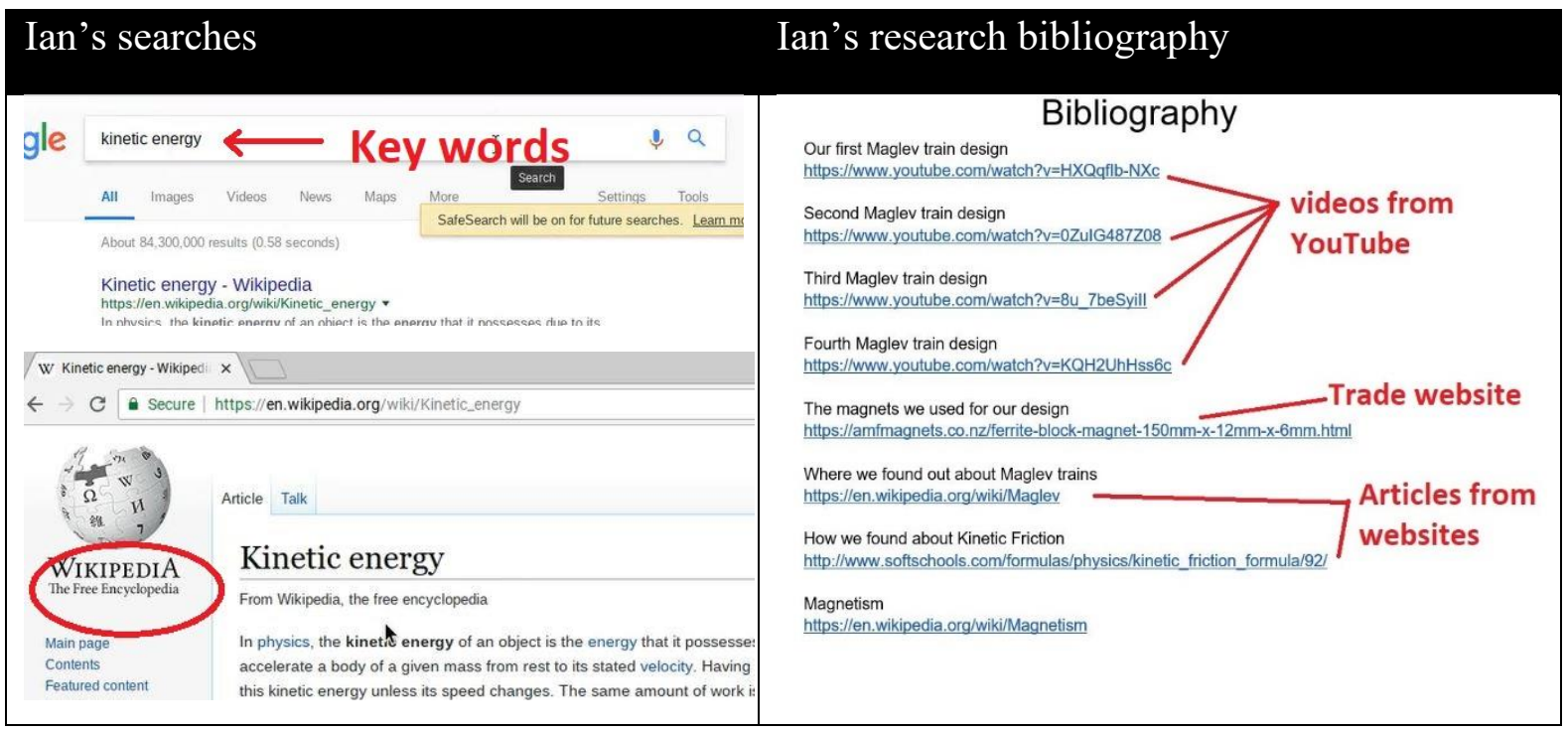


In general, the participants spent a considerable amount of time 'interactively' searching for information in the scientific research activity and Year 8 leadership activity, while the use was more passive in the leadership activity for Year 7 participants.

\section{Shaping Using Devices as a Source of Information}

Tess believed that learning involves different things; one of them being that "It can be about gaining information like facts and figures and knowing about history and that kind of thing". She said that one of the reasons for using the devices in the learning activity was "doing research on the Internet". She believed that students learn best when they are given more opportunities, options, and control over their learning. She thought that using devices as a source of information provided more options and variety.

Tess said that one of the factors that enabled using the devices for searching was "teaching digital citizenship. So we have specifically taught them how to do safe searches or how to keep themselves safe when they're on the Internet'. The observations supported that. During the learning activities, some of the instructions that were given to the students were general (Figure 5.4, below). These instructions asked the students to 'Search' and cite their references without specifying the sources. In this case, the students had a choice of sources. For example, Bob, Ian, and Alice used different types of sources, while Mitchel chose videos. Other instructions directed the students to specific links or resources to be used.

\section{Figure 5.4}

\section{Teacher's Instructions to Use the Devices as a Source of Information}

\section{Directions to use unspecified sources}

4. Research the answers and make notes in your log book. You must write down the name of websites/books you get your information from in your log book for your bibliography.

\section{Directions to use specific sources}

Click for an: Example of how to write your Experimental Procedure

Checklist for Experimental Procedure

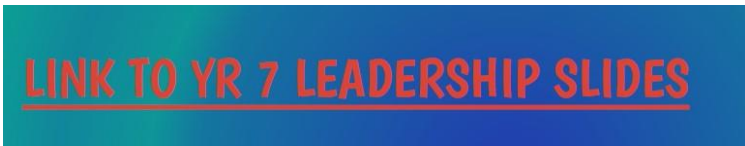


Tess gave the students strategies to help them research their topics. Bob said, "I think I got better in finding what I needed on the computer and making sure we had a worksheet that helps us with questions for our research". Bob referred to a worksheet that included instructions about how to organise her research to develop her research questions and hypothesis (Figure 5.5, below). These instructions addressed the logic of the research process. At the same time, the techniques of using the search engines were left to the students. There was no specific technique used by all the participants; for example, Bob used questions, and Ian used key terms.

\section{Figure 5.5}

Instructions Relating to Using the Device as a Source of Information

\begin{tabular}{l} 
STEP \#3 Now decide which question interests you the most. In choosing your \\
topic, be sure to not take on more than you can handle. \\
- Does the topic sound interesting to you? \\
- Is the topic interesting enough for you to read about it before you start? \\
If it is an Experiment: \\
- Can you get measurements or some kind of number for data? \\
- Can you measure a change in the variable studied (dependent variable)? \\
- Can you collect a lot of data? (20 or more numbers) \\
- Can you change the other variable (independent variable)? \\
- Do you have all the materials you need, or will you be able to get all the \\
materials quickly? \\
- Do you have time to do the experiment twice? \\
- If the project involves human subjects, are you willing to get permission from \\
every adult and parent permission for every student tested? \\
If it is a Research project: \\
\hline - Can you create a physical model to show what you learn?
\end{tabular}

Digital devices enabled the participants to access different sources of information. The search engine finds a huge number of articles that can be used for student research. Alice said that the Google search engine was "helping a lot". However, she considered that the hardest part of her scientific project was discovering the science behind the camera. She said it was difficult to find sources about the topic. She felt that she needed to do more research to be able to present her project in a good format. After hard work, she and her friend were able to find information that enabled them to answer their research question and design a model of a camera obscura. Mitchel reported another problem: "well when we like research stuff it didn't come up with the thing we're looking for. It came up with like a different thing. We actually just keep them researching different things". Bob complained that there was a lot of unnecessary or imprecise information on some popular websites such 
as Wikipedia, from her point of view; suggesting another simpler version, she described her experience:

I think Wikipedia is unhelpful. Oh, I learned that Wikipedia is unhelpful. Because it's way too many words and then so you'll search up. Like I searched up the Māori gods on Wikipedia once and it had like only like three of them and then it's somehow made a two page long writing about two or three gods and it was like what?!! It just overcomplicates things and makes you more confused than when you started.

Therefore, even if the search engines were useful, there was sometimes a lack of information or confusion depending on the existence or the quality of the sources on the web.

There were other helpful features: when Bob found a video about one of the scientists, she could not spell his name correctly as she had heard it from the video. The search engine suggested the correct spelling, which enabled Bob to find the information (Figure 5.6, below). In addition, Ian and other participants used the links and the pop-up bars embedded in the web articles (Figure 5.7, below) while searching the web. The browser history was helpful for Bob, both to search for information and to save her own information.

\section{Figure 5.6}

Suggested Spelling in the Search Engine

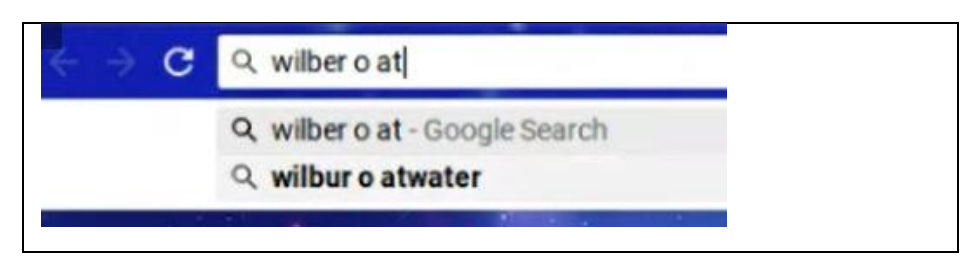

\section{Figure 5.7}

Ian Using the Pop-up Feature

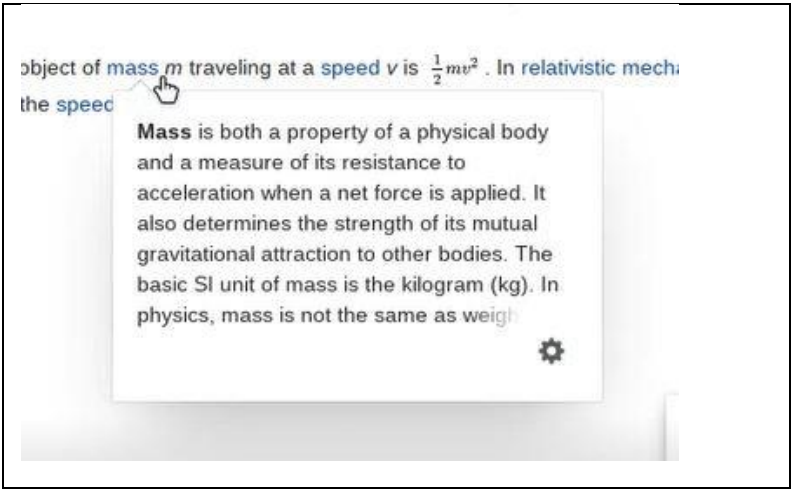


The students believed that devices were efficient as a source of information. Alice said, "they are the easiest form of information that we have available at school". Ian said as well, “The internet is just so huge. There's so much information. I think we couldn't be able to do it without it". Bob said, while working, "It [Internet] will give me an answer rather than needing to search the books and things. It's actually really helpful, and then I can just copy and paste something into here [her Google document] because I don't need that much detail". Even Mitchel, who denied learning gains from the activities, said that "researching stuff" was the main help he got from the devices.

In addition to their beliefs, their digital skills and knowledge about sources influenced their use. Ian said that it was easy for him to search the web. Observation showed that he was able to use appropriate keywords and locate suitable websites while searching for information. Although he browsed different websites, Ian and his group included the most relevant sources in the bibliography of their research. Alice showed competency in searching the web as well. Tess commented, "[Alice $]$ uses the computer and the Internet a lot, so I'd say she's got a lot of confidence. Probably, and being able to access information on the computer and things like that, would definitely be supportive". Bob had digital skills and knowledge of how to use his search history to locate information, as illustrated in the use section.

Additionally, Bob, Ian, and Alice were able to incorporate different sources of information from the Internet, such as articles and videos. For Mitchel, reading was challenging; therefore, he limited his sources to videos and did not benefit from sources in text format.

How students responded to teachers' instructions about searching for information was another factor relating to students. Alice commented while searching that she needed to look for good sources of information and targeted scientific websites. Bob used appropriate questions to locate information, copied them, and placed them under the questions in her document. She followed her teachers' instructions on how to locate information and evaluate the websites, which helped her in organising and managing her research. Ian developed his own way to organise information he found; he created three documents, each related to a certain topic, and then added the relevant information for each of them.

Student self-control played a role when dealing with distraction, which was one of the challenges the students faced while searching for information. Bob, who showed commitment while working on the devices, complained that her peers easily got distracted when they started searching for information. While working, she regularly reminded her peers not to get 
distracted and to focus on searching. That was not the case with Mitchel, who easily got distracted while searching for information.

\section{A Means of Communication}

The students used the devices as a communication tool in the two learning activities. During the leadership activity, the students communicated through the devices with each other and with people from inside and outside school. Alice reported:

We were able to contact people that we needed to, like for piano before assembly, we could email the people who wanted to do it, and we could like update notices ... we could also contact people and talk as a group.

For Year 7 students, the students communicated through and about using the devices. Bob and Mitchel contacted the teachers in the school to send them Google forms to undertake their surveys (Figure 5.8, below). In the previous term, they communicated through emails. Their leadership lessons included how to use emails and Google forms. During their work, Bob discussed different features with her peers, such as using the multiple choice option in their Google form. Mitchel was observed discussing creating the forms with his peers. There was also communication about computer games that happened between Mitchel and other students.

\section{Figure 5.8}

Screenshot of Bob while sending a Google Form to a Teacher in her School

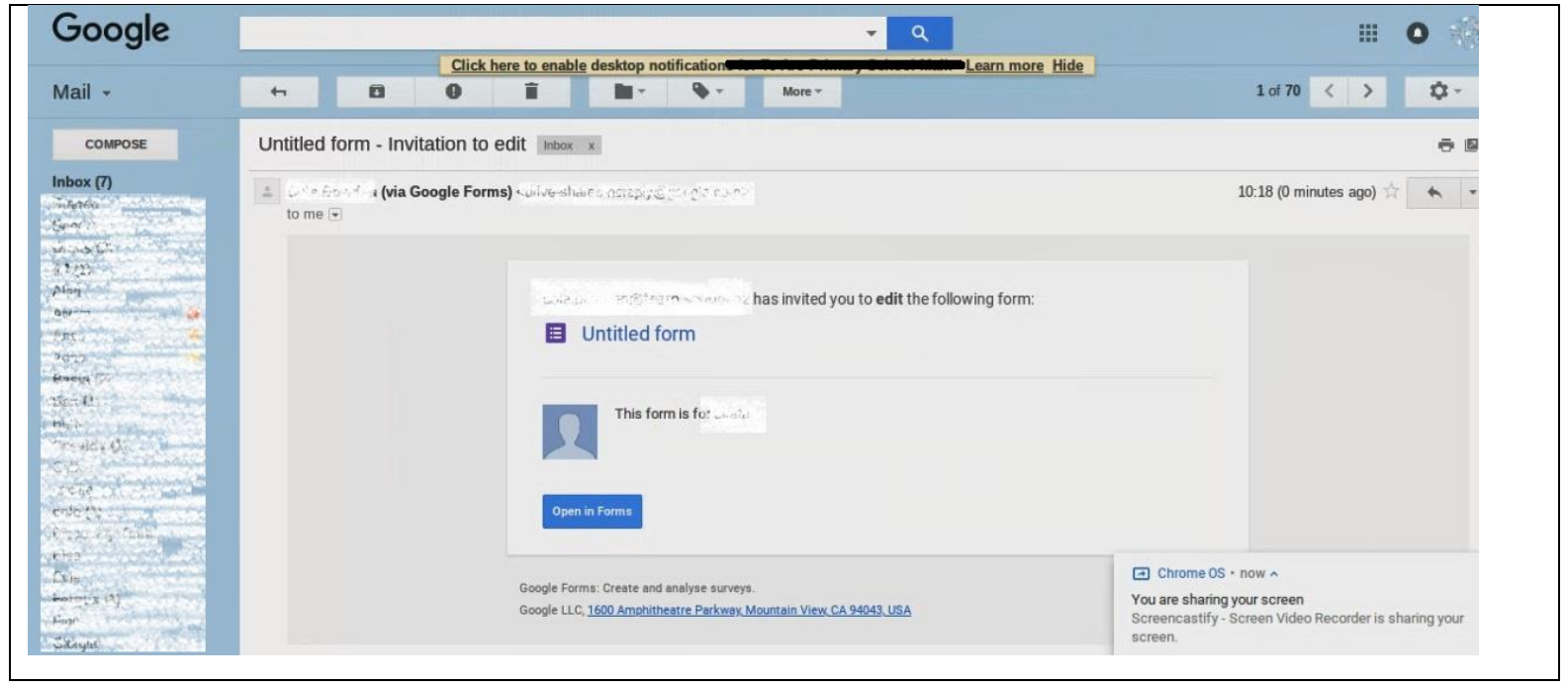


In scientific research, the students communicated with each other through the devices. Ian and Alice reported that they exchanged emails with their group members to complete their science projects. Ian said, "We could do a lot of work at home as well as working with each other. So we didn't have to be in the same room to communicate and to work". Alice reflected while working, "I'm trying to find some stuff for the science fair that [her friend] sent me so that I can click on the links and see".

Student use sometimes surprised Tess. She had a group of three students that included a student with learning difficulties and English was the second language for the others. She said, "That was the biggest surprise" because they were the three most unlikely students to finish their project early. She reported how the group leader used devices for communication after school and they were able to participate in a science fair:

He used [text messaging app] to organise to meet the rest of his group at the public library. And organised to get himself there in the weekend and they all meet up, and they planned and prepared their work, and I think that they have met up since then outside of school as well, which is amazing, you know, on so many levels. One that he would even find a [text messaging app], that he'd be able to find the others, that he'd be able to organise to get himself there and get things ... you know, to do all that organisational stuff was pretty amazing.

In school the students were allowed to use translation apps such as Google translate for communication or to do their tasks.

Tess reported communicating with the students through the devices to give feedback as a common practice in her classroom. She said:

If I want to be able to access their work while they're working on it to give them feedback. So for example, if they're working on a Google doc or a slide show or something and they're going to want feedback from me, then it can be much more efficient if I can give them direct feedback then having to collect their books.

This shows that giving feedback through devices was adopted by Tess because it provided a fast and efficient way to communicate with the students.

\section{Shaping Using Devices for Communication}

Tess encouraged student communication through devices for academic purposes. When Tess was explaining what learning means she said, "I think that learning is making connections". 
She added that it could be "about how to behave and how to interact with other people". Student research groups and leadership groups organised their work and meetings using social media apps and emails. That kind of communication was not always supervised by Tess, but it was encouraged, and sometimes surprised Tess as reported in the uses section.

In the leadership learning activity, there were lessons and detailed instructions on how to communicate through the devices for Year 7 students. Tess said, "They've been taught how to send emails appropriately and how to write them." This included communicating with their peers and adults. The lesson included different aspects of communication by email; for example, but not limited to, the rules of using email such as safety and privacy, how to write and send emails, and how to evaluate emails. The lessons provided samples of different types of emails (Figure 5.9, below). The students practised sending and evaluating emails with the teacher. These lessons represented communicating about the devices as well.

Applications such as emails and social media apps enabled the students to communicate. In return, the students had the skills to use these apps and take advantage of their features either by learning in school or learning on their own. The harmony between the groups was also found to influence students' communication. Ian, Bob, and Alice communicated effectively through the devices with their group. Ian's group communicated in school and outside school. In the leadership activity, Mitchel was observed communicating about creating the forms with his group members, and they were engaged. However, he said that he could not engage with the science group members and there was a lack of communication between them. 


\section{Figure 5.9}

Instructions for Using Devices for Communication

\section{Rules around using emails for communication}

\section{Email Etiquette}

There are expectations and rules around sending and receiving emails including:

- How to write/compose an email to someone first

- How to reply to emails you receive (and when you should)

- Privacy

Some of these rules and expectations will be different for different people.

When you are writing an email to someone for the first time, what do you think are some of the things that will be the same when writing to anyone?

\section{Samples and tools for evaluating emails}
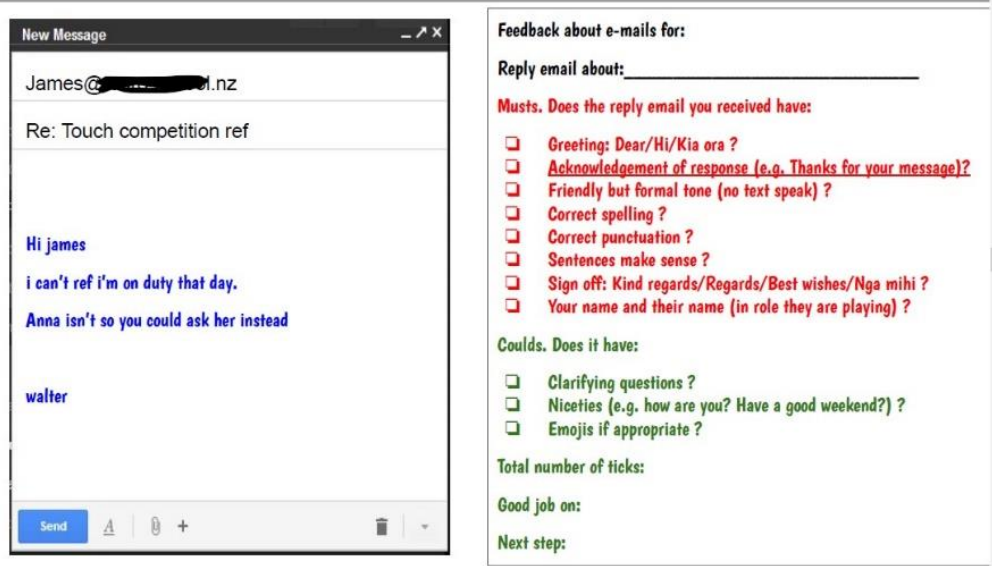

\section{Training the students how to send and evaluate emails}

To do today:

1. Choose a person and a scenario from the lucky dip boxes and write an e-mail to them (but send it to your buddy). Remember to CC in

2. When you receive an e-mail, from your buddy group, pick out the choices from the lucky dip boxes, send a reply and remember to hit Reply All.

3. Reply to the reply (so there are 3 emails in the chain).

4. For the 1st replies you receive from your buddy group, complete a feedback form and give it to them.

5. Read the feedback you get and start again!

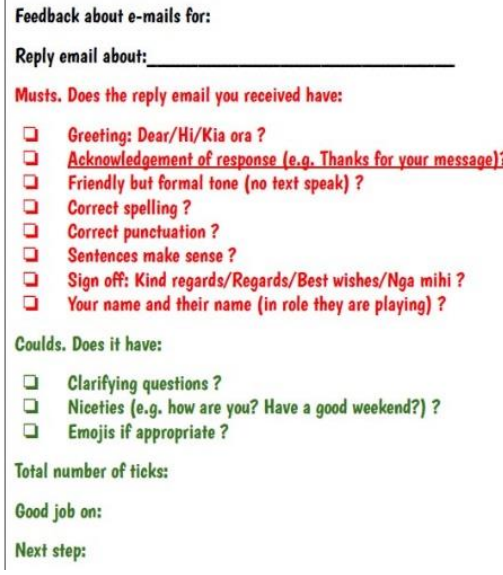




\section{A Research Tool}

This way refers to specific ways of use all related to research activities. That includes using the devices for data collection and recording, testing, and experimentation, and analysing. The following are examples of this type of use, which happened occasionally and for limited purposes.

Collecting and recording data: In the leadership activity for Year 7, Bob and Mitchel used their devices to collect data. They created Google forms, then sent them to the teachers of different classrooms to answer a survey about physical education (PE) gear needs. Bob said, "we sent one to each class, and it's about what sports equipment they want in their class? How many people want basketballs? How many people want soccer balls? And then they put down the number". That way worked with some teachers, but other teachers did not respond. Bob thought of another way to collect the data. She and her peers went to the classrooms holding their Chromebooks. She asked the students the questions of the survey then filled it out directly on her Chromebook.

During Bob's scientific research, she and her group wanted to do an experiment. The procedures included different steps as seen in Figure 5.10, below, such as presenting the food, asking questions, measuring, and recording. Bob was holding her Chromebook and directly recorded the measurements of step 4 on her device. Bob said it was easier and faster to directly record the data in this way. Tess reported that many students used their Chromebooks to record their data, which shows that children replaced traditional ways of recording data such as using pen and paper.

\section{Figure 5.10}

\section{The Procedure of Bob's Scientific Research}

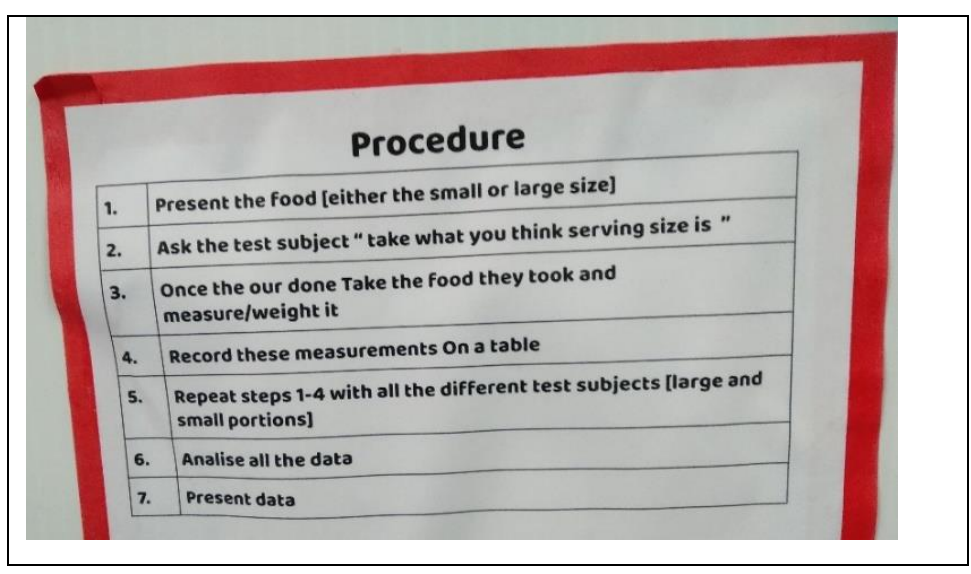


Testing and experimentation: Tess said of students' presentation and preparation of the testing that "nearly all of that occurred on devices". Mitchel's experiment was about reaction time after playing a computer game. As in their procedures in Figure 5.11, below, the participants in their research were required to play a computer game using the device and then record their reaction time after that. They documented their experiment by taking a photo of this testing and added it to their presentation board.

\section{Figure 5.11}

The Procedure of Mitchel's Scientific Research

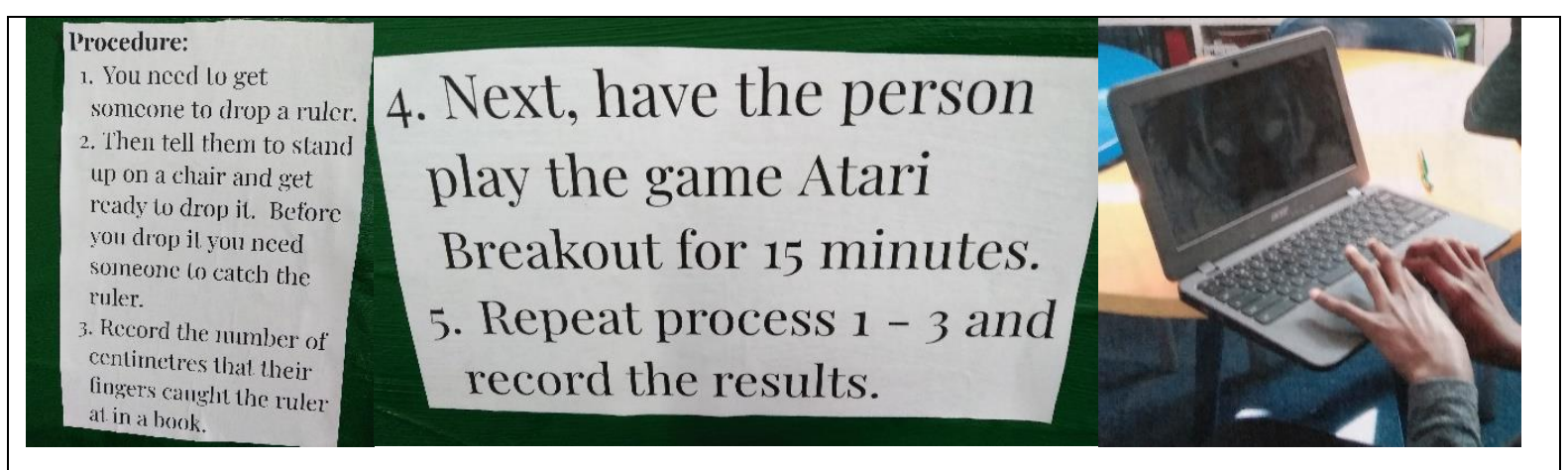

Mitchel's group was not the only one; Tess reported other examples:

Two of the boys were doing a test that involved bike riding. And so they used digital devices for timing the riding of the bike and also taking videos of the test, taking photos of the test. They've all taken photos of the testing.

That was observed in Ian and Bob's groups, which took photos of their experiment. Tess reported an unexpected way of using the devices for testing:

One of the groups ... were doing a test about bouncing a basketball on different surfaces and they couldn't work out an accurate way of measuring the height of the bounce. So, they decided in the end that the best way was to take a photo of it. So, they use photography, which was great.

Tess clarified that the students used their devices to measure the height in the photos.

Data analysis: Bob and her group used their devices to analyse their data. They created tables and charts using spreadsheets, as in Figure 5.12, below. 


\section{Figure 5.12}

\section{Using Devices to Analyse Data by Bob}

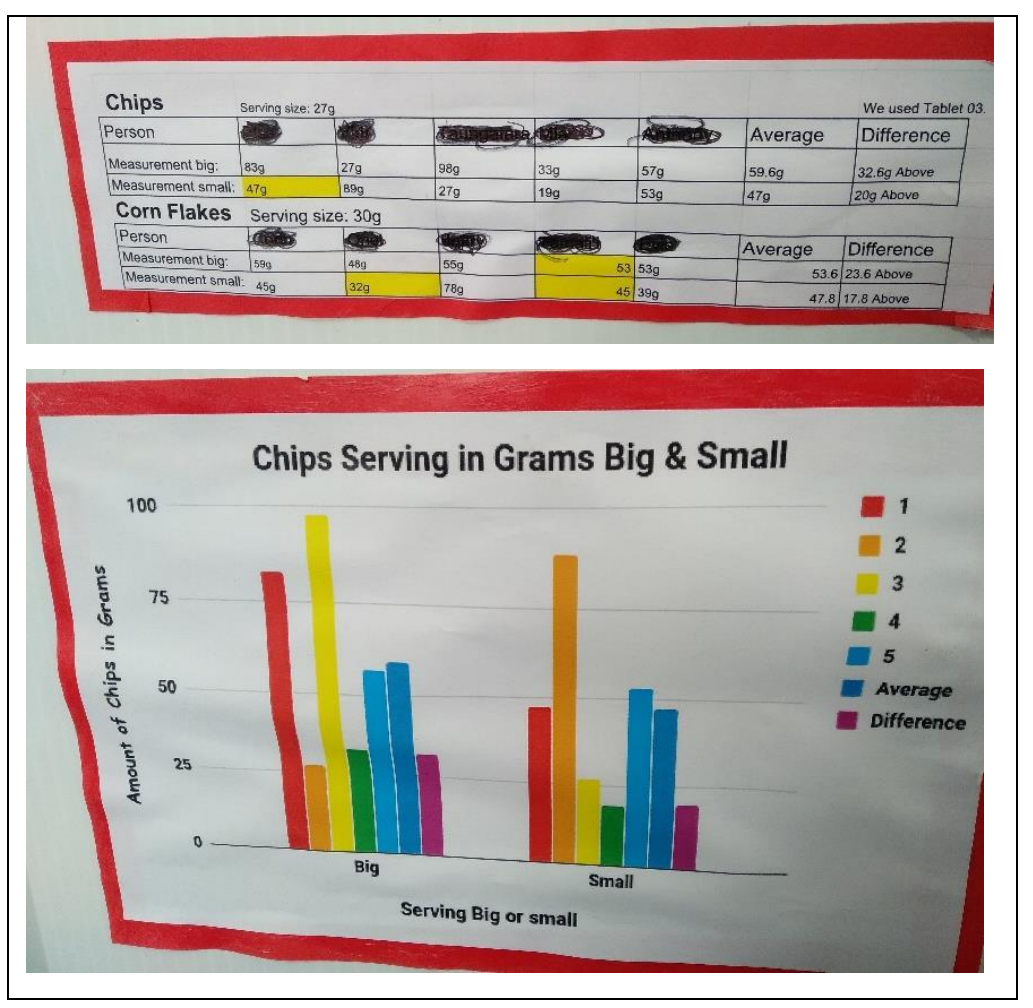

\section{Shaping Using Devices as a Research Tool}

Using the devices as a research tool was influenced by teaching strategies. Tess reported that they gave lessons on how to use devices to collect data:

There's an element of actually specifically teaching them to use the devices. So it can be, for example, introducing different Google apps. So just recently the Year 7 students have been learning to use Google forms in order to do surveys and collect information. So that was a specific skill that was taught this year.

During the activity, Year 7 participants took lessons about how to create and use Google forms and for what purposes (Figure 5.13, below). The lessons provided step-by-step instructions that were supervised by the teacher. In addition, there were instructions about using devices for data analysis, taking photos to document their research experiments, and planning for their next steps. The school environment allowed the students to move freely in their classrooms and between classrooms, holding their devices to collect data, take photos, do their experiments, and work in groups. 
Special applications were useful for using devices as a research tool, such as Google forms. Bob was observed using spreadsheets to analyse her data; she said they were quick and easy. She mentioned that she learnt how to do data analysis in maths class. From what Tess reported, it can be concluded that some experiments required scientific knowledge.

\section{Figure 5.13}

Instructions on Using Devices as a Research Tool

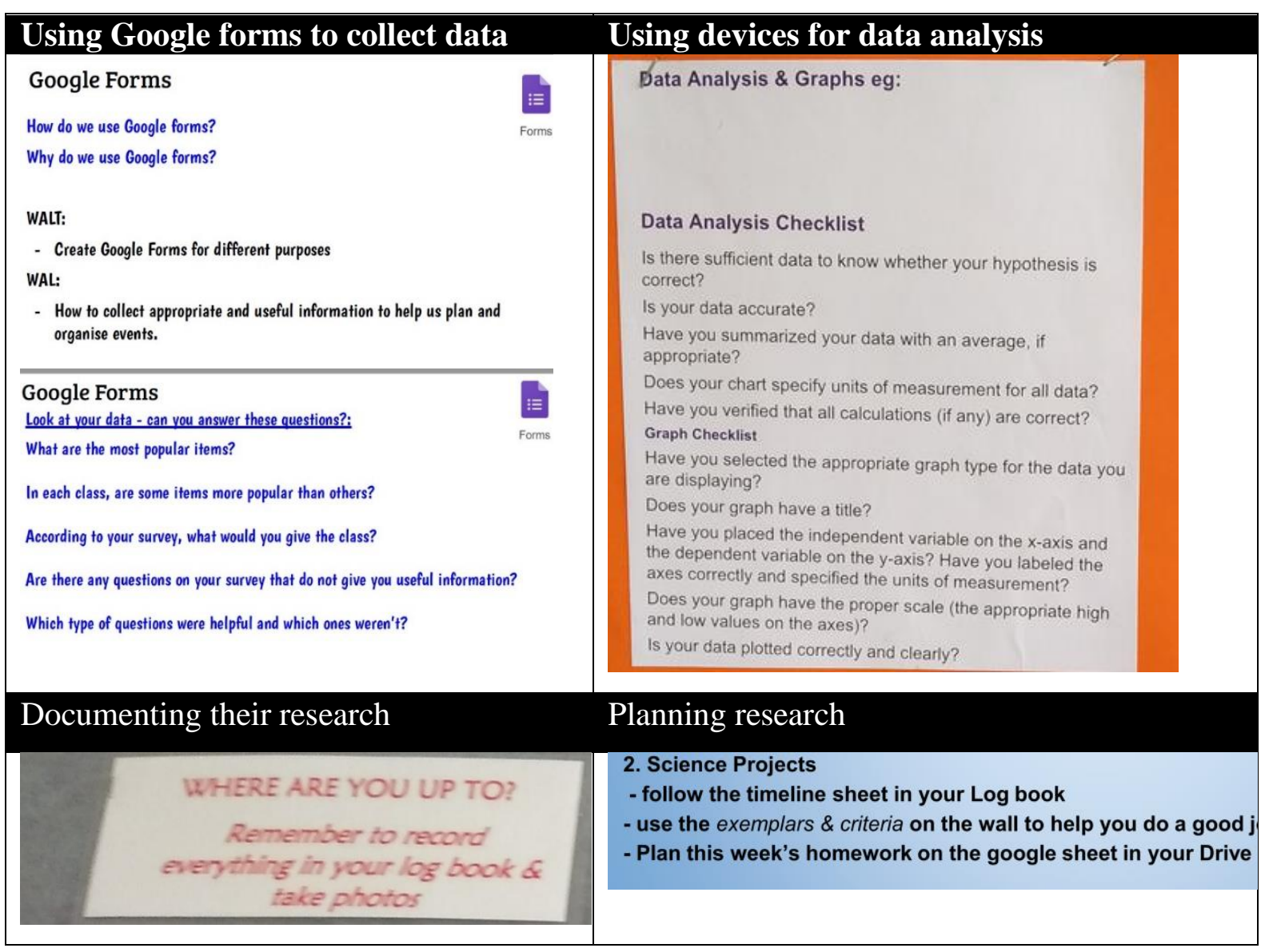

\section{A Production Medium}

In this class, the students used the devices to produce digital and non-digital products. All the products were collaborative. In scientific research, the students were required to produce a display board that represented their research. The students first designed everything on their devices (digital product). Then they printed out their work and put it on the display board (hard copies). In Bob's display board different types of digital products were presented including tables, charts, photos, and text (Figure 5.14, below). This was seen in almost all students' display boards. Tess reported that most of the groups took photos related to their projects and used them in their presentations. Mitchel and his friend took photos and wrote 
paragraphs to be glued on a display board. Alice and her friend printed out their research and glued it on a model of a camera obscura that they had designed.

\section{Figure 5.14}

Bob's Display Board for the Science Learning Activity

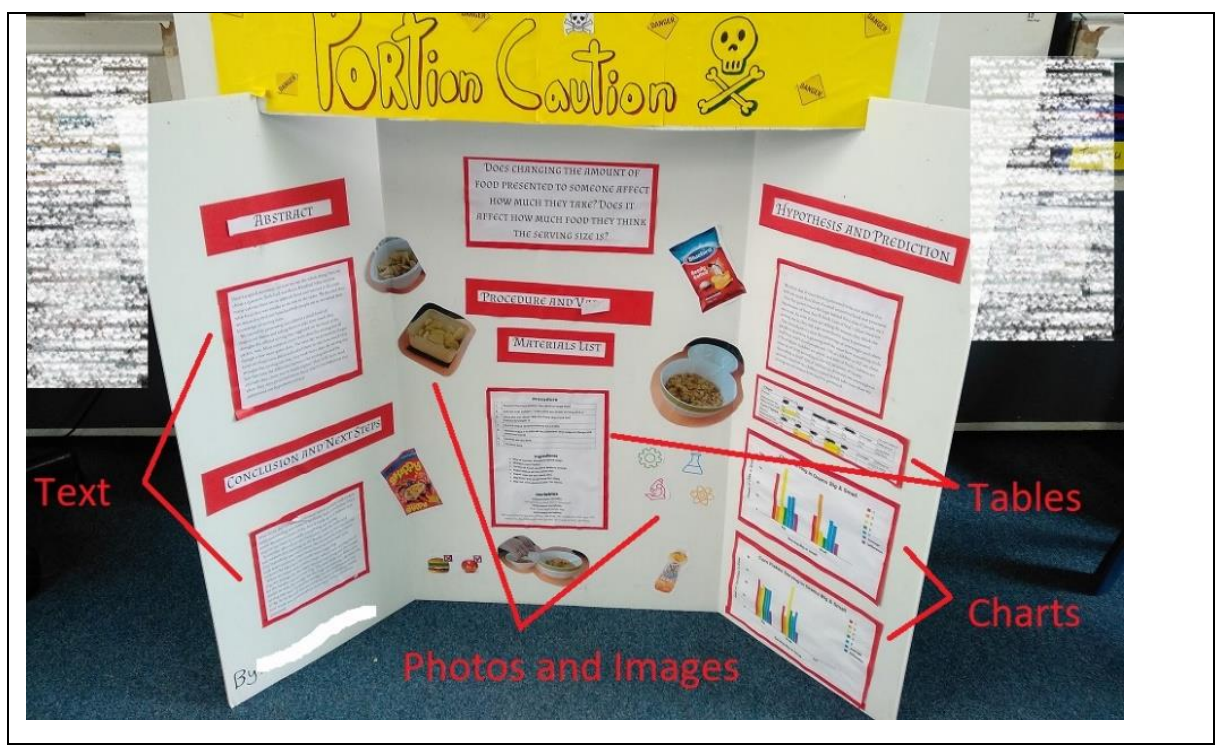

Ian's presentation contained text, images, and diagrams from the Internet, and photos they had taken. These products were digitally developed then printed out and were glued on the display board (Figure 5.15, below).

\section{Figure 5.15}

Ian's Display Board for the Science Learning Activity

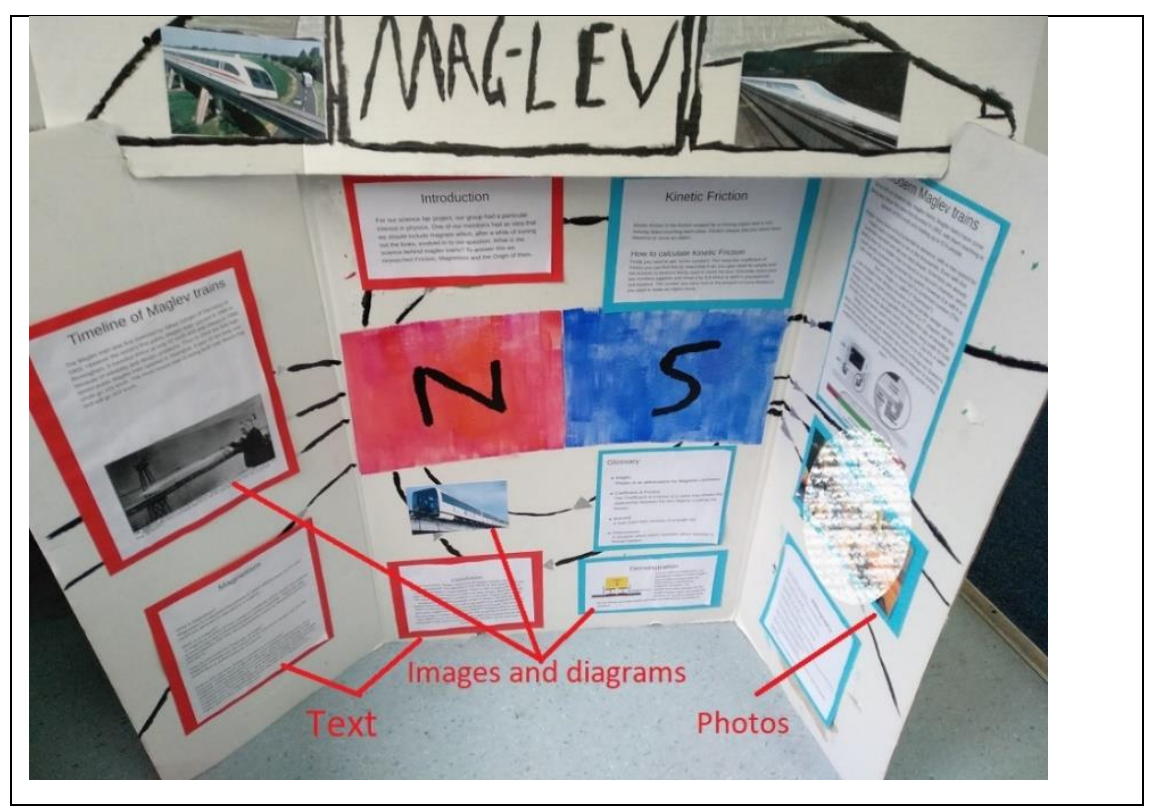


In the leadership learning activity, the Year 7 students, Bob and Mitchel, were required to produce Google forms to collect data from other classrooms, as in Figure 5.16, below. Tess gave examples of Year 8 students' products:

There's one group that has started creating the Year 8 leavers' book for the end of the year, like a yearbook thing. So they've already started working, and they're creating it in Google slides ... and alongside that are also producing a fortnightly school paper, a little mini-magazine.

She said they were "producing those on their digital devices ... so the Year 8s are doing different things depending on what group they're in". That shows that the devices were used to create different types of products for different purposes.

\section{Figure 5.16}

\section{Bob's Screen Capture while Creating a Google Form}

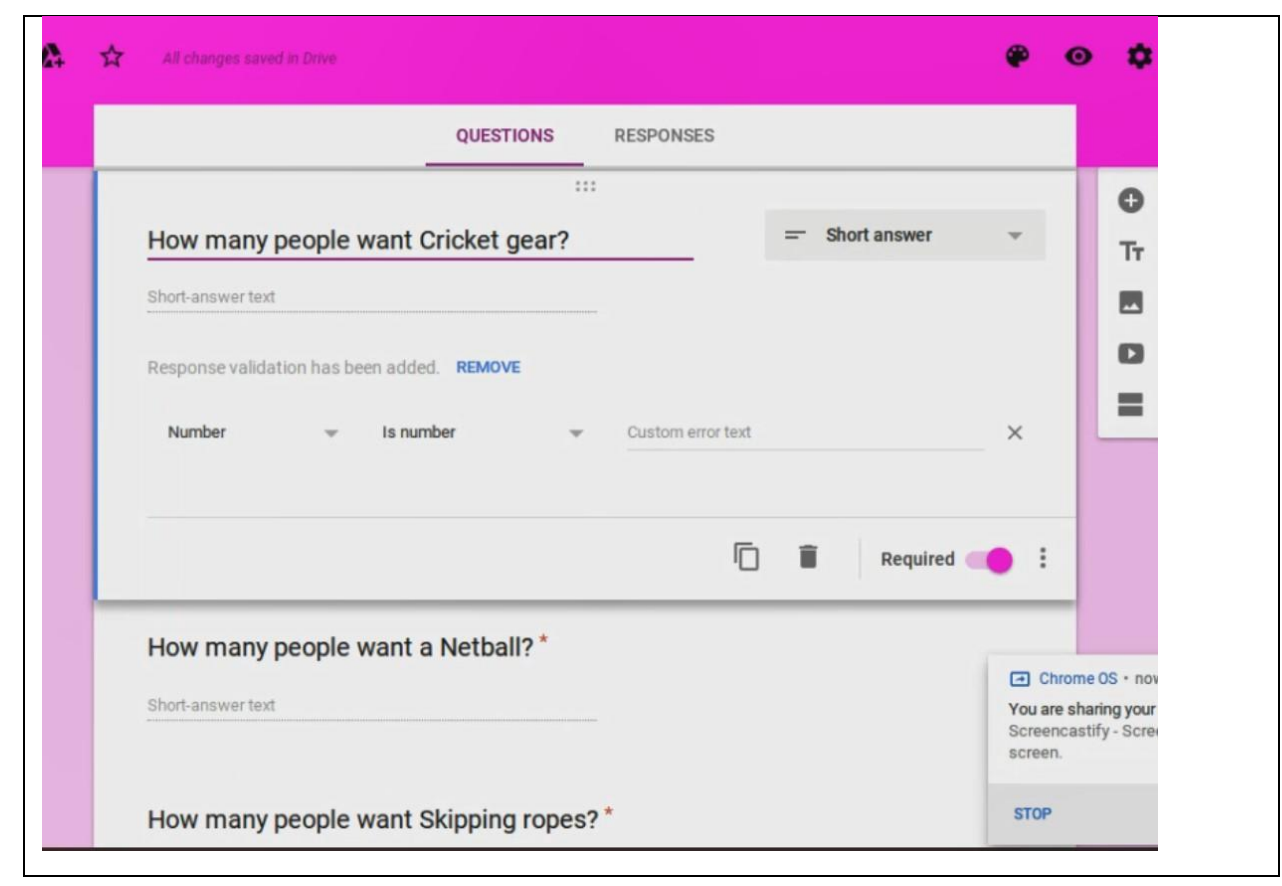

The final products resulted from a combination of the other uses, which were communications, as a research tool, a source of information, and as collective memories. They were then saved either in their personal or collective memories or to create non-digital artefacts. 


\section{Shaping Using Devices for Production}

Tess's beliefs about learning and teaching were reflected in her pedagogical choices. One of the meanings of learning that Tess stated was "developing skills that enable you to do things like reading and writing or cooking or sewing, building". She believed that digital technologies gave more options to the students to present and illustrate their learning and show their skills. Her pedagogy included different strategies that enabled using devices for production. Tess said, “We've done quite a bit of work around that. So, I guess it's either that we are teaching them to use a variety of digital devices specifically or we're using them as a tool in one way or another". Furthermore, Tess pointed out that using the devices will depend on the activity; she gave an example of the Year 8 leadership activity:

They [the students] are producing those [projects] on the digital devices ... so the Year 8 are doing different things depending on what group they're in. So for example, there's ... the music group and they'll be running assemblies. And so their use of digital devices would be different. It would be collecting music to play in the assembly and that kind of thing. So it depends on the activities.

Tess provided detailed instruction on what students' products should look like, such as the display board for scientific research (Figure 5.17, below) or Google forms. These instructions did not explicitly indicate the need to use the devices, but Tess emphasised the role of the devices in this activity: "doing that research and then ... definitely using those [devices] to present". All the groups designed and printed out the components of their display boards using their devices. On the other hand, Google forms were digitally produced, so using devices was necessary. 


\section{Figure 5.17}

Instructions on Producing Scientific Research Presentations

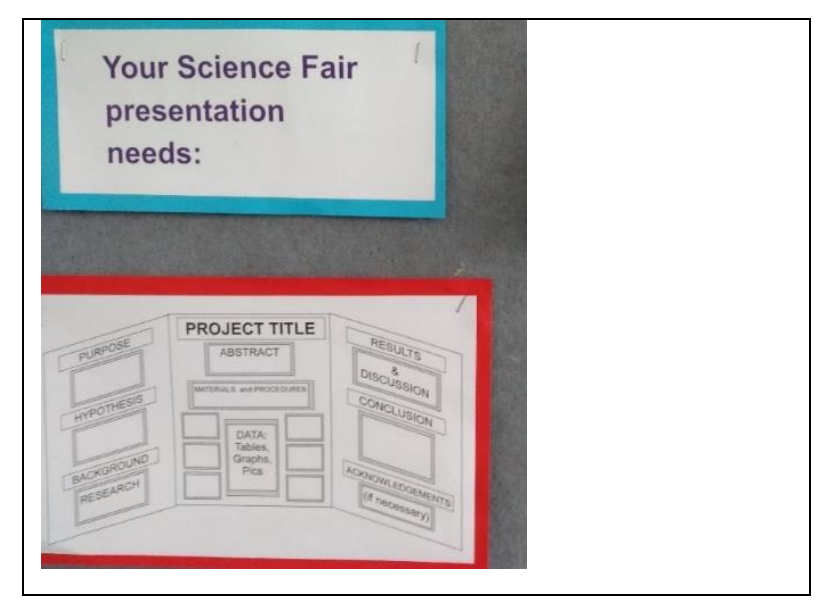

Tess reported that it could be sometimes challenging to supervise using devices when students produced their work as a group and to ensure that all of them were engaged:

[Teachers provide] some really specific direction about what they're [the students] doing when they're in that role. So for example, dictating that each paragraph that's written must be typed by a different person, but you have to get down to such a gritty, even though when you sign in to the document, making sure that it's not always the same person that signs in because kids are very good at covering up and it can be the whole year's gone by before you realise that a particular student still can't log in to the Gmail account or something.

All products in observed learning activities in Tess's class required collaborative effort. Therefore, Tess's supervision played a role to reinforce the participation of all students in the production process. In addition, the classroom environment enabled the students to communicate and make a reasonable amount of noise to work collaboratively while using their devices.

Time management of the activities influenced the production process. Having more time would enhance their final products. As Ian said, "We also haven't time to check over our documents as we found like grammar mistakes". Tess agreed that there was a problem relating to time management, as they needed to replace activities with others.

Tess mentioned some features of the devices that enabled the production use for the students with learning difficulties: 
I think accessibility and a variety of different ways. So, for example, the obvious things like voice typing for kids who find writing difficult. Or accessing information by watching videos rather than reading for kids who struggle with reading. Or recording their ideas orally rather than writing them down.

That, for example, was very helpful for Mitchel, as discussed earlier. The other feature relating to the production medium usage was the ability to combine different elements in students' work. Tess said:

The fact that it's easy to incorporate lots of visual material. So, you know, finding a picture on the Internet and instantly putting it into your work. What else? I guess, for example, being able to do work through photography and video, which previously was quite difficult just to manage in school.

The participants had digital skills and knowledge that enabled them to use a wide range of features and apps. They used different applications for production, such as Google forms, word processing, spreadsheet apps, and photo editing software. They created tables and graphs, and used editing tools such as font, font size, colour, text alignment, and spell check to produce their documents. For example, Ian was very fast in typing and mastered using many applications such as slideshows and word processing apps. They were able to take photos and edit them to be used in their projects and were able to use the school's printers independently.

Students' attitudes, capabilities, and interests influenced the production process. Ian, Bob, and Alice were active during the discussions about the production process with their groups; they discussed how the final products should look and how to distribute the workload between them. They worked independently most of the time, as they had done scientific research before. Mitchel and his peer received direct supervision from Tess and a teacher aide, as that was their first time to making a science project. In addition, Mitchel needed that help because of his reading and typing challenges. Mitchel preferred sound recording applications for typing. He said that he was slow in typing. He said about the scientific research, "there is a lot of typing and looking up stuff on the computer. You have to do a lot of reading and much on videos". For Mitchel, using some features in digital devices was helpful to overcome these difficulties. Finally, scientific research projects reflected students' interests: Bob's interest in nutrition, Mitchel's interest in gaming, Alice's interest in photography, and Ian's interest in physics. 


\section{An External Personal Memory}

As discussed in the first case, this use means that the devices provided personal and private places for the students to save, access, and retrieve digital artefacts, which could be created by them or by others. In this case, the students regularly saved information they collected for their scientific research on their Google Drives. They also saved their digital products such as, but not limited to, developed texts, tables, photos, and graphs. Ian and Bob were observed opening their drives to retrieve documents while working on their projects.

An interesting way of using the device as an external personal memory was observed while Bob was working. Bob was searching for a specific resource she thought was important to be added to her resources (Figure 5.18, below). It was about an early scientist who studied the calorie content of the food. She could not remember his name, so she first tried to search in websites that she might have visited before. She started looking at the search history of her Google account and checked the search history on other devices, that is, her device at home. Then she checked the search history of her YouTube account; finally, she was able to find the information. Her personal accounts and search history provided an external memory that recorded her research, so she was able to retrieve information when needed. 


\section{Figure 5.18}

Bob Using her Search History to Find Information

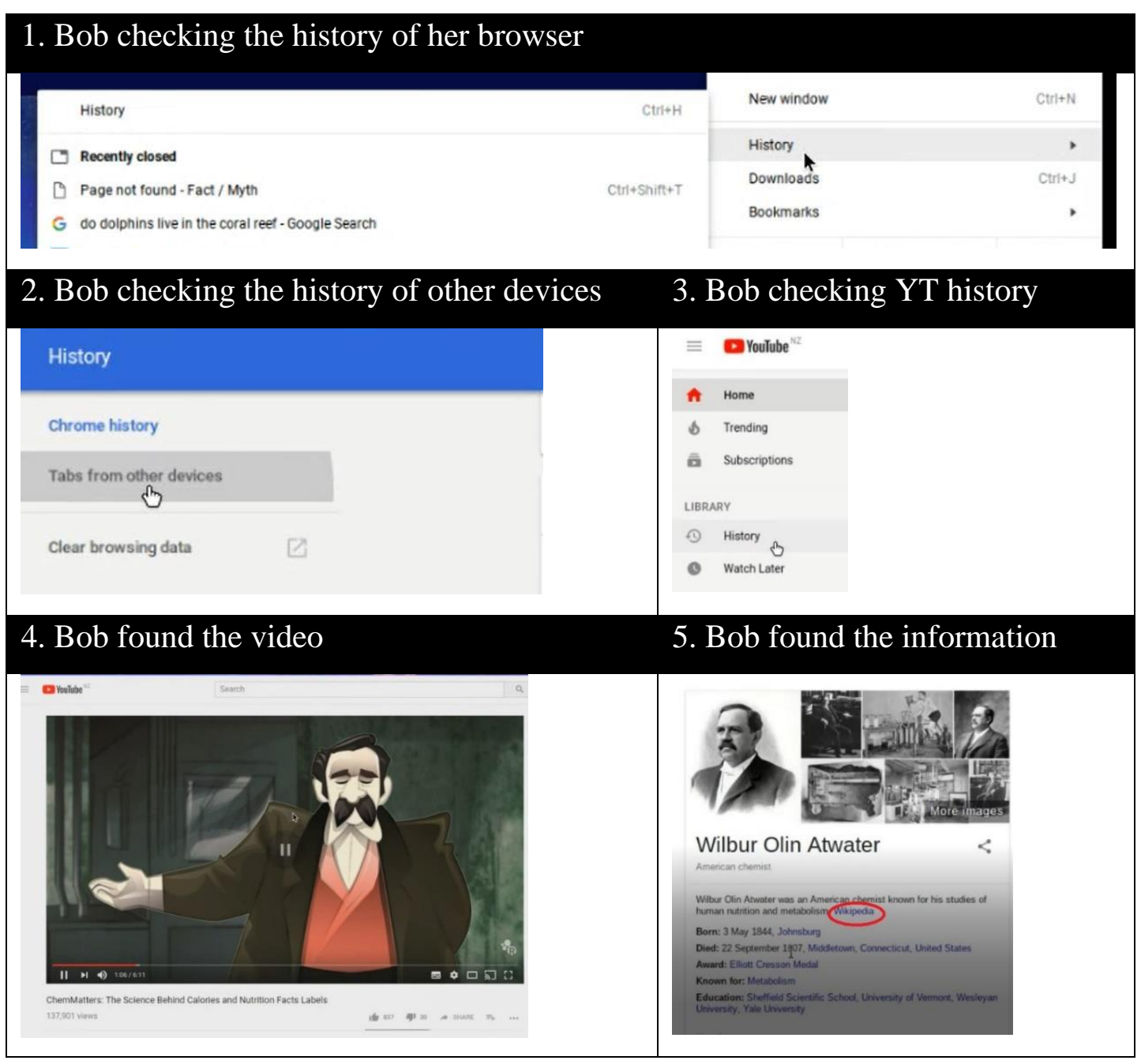

\section{Shaping Using Devices as Personal Memory}

On the classroom wall, there were instructions about how students should manage their personal and shared accounts (Figure 5.19, below). In addition, teachers gave the students lessons about how to organise their own drives, including how to log in and how to organise their folders. The participants had the essential skills to use the devices in different ways as they started learning these skills early. Ian said, "I had Google accounts ... since Year 6 or two years. And I've been learning how to use those really well, move quite quickly between things and a lot of shortcuts". Therefore, when reaching Years 7 and 8, this type of use occurred spontaneously. Bob showed competency in using her search history to remember the location of certain information. Ian appreciated the saving features and said, "By having access to the internet and just find what we needed and Google docs, and being able to record it all in one place". 


\section{Figure 5.19}

Instructions Related to Managing Personal and Collective Memories

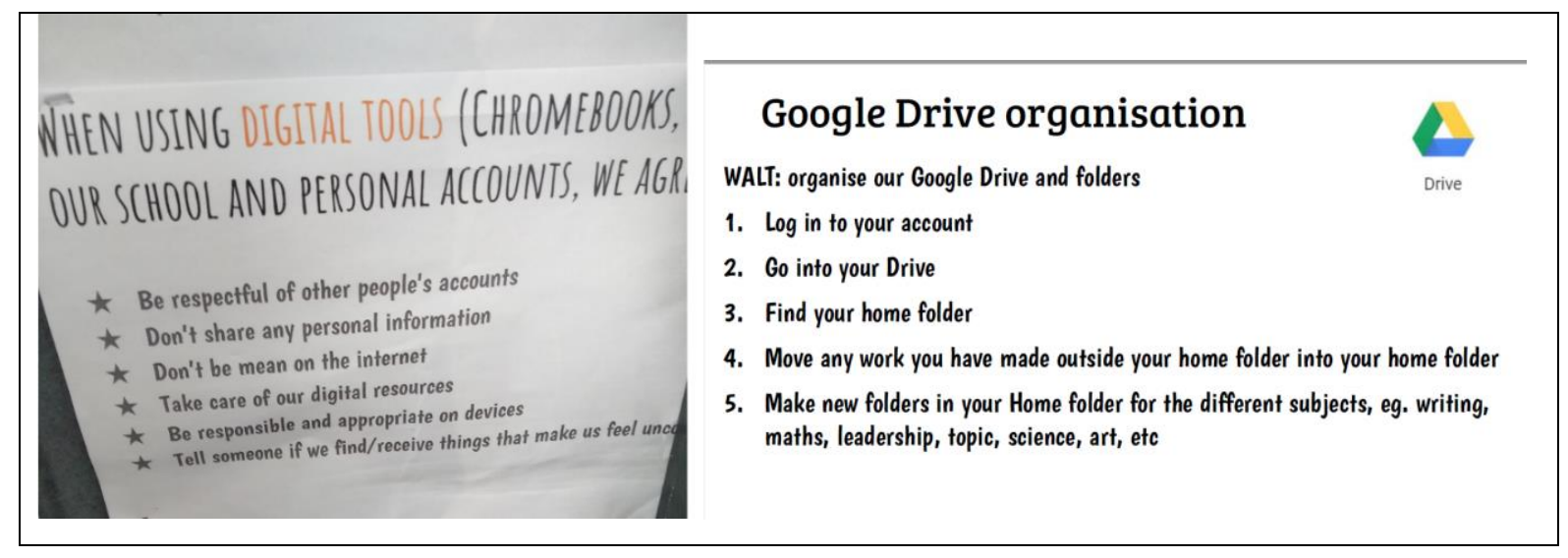

\section{A Collective Memory Coordinator}

As in the first case, the students created documents collaboratively where they could save, access, and retrieve information. Scientific research and the leadership activity were collaborative activities. The students constantly accessed their shared information while working. An example of creating a shared document happened during the leadership activity. It was not planned, as many students from the enviro team left the school. Therefore, Tess asked the students to form a new team. Ian volunteered with seven students. They sat with Tess in a circle holding their devices and I sat behind Ian to observe his screen. Tess created a document that was shared and accessed by all eight students. Tess discussed with the students "climate change and what can they do about it". The students were required to reflect on this issue and write down their ideas. The students started writing their ideas while chatting with each other. I was able to see Ian correcting spelling mistakes for what was typed by other students. Other students were changing the fonts and the colour of the text. Tess was organising the discussion and encouraging the editing process. By the end of the discussion, a document was developed that contained collective ideas from the students and was saved in a shared folder. After that, Ian went to his table and used this document to create a slideshow for the team. Alice's music team had shared folders and documents to organise their activity as well.

In scientific research, Bob was developing a document with her group members to highlight the main questions and the references to answer these questions. Her peers in the same group were also typing at the same time (Figure 5.20, below). This document was then 
saved so all the group members could retrieve it to edit. It was used when the students created their final presentation on the display board.

\section{Figure 5.20}

Bob while Creating a Collective Memory

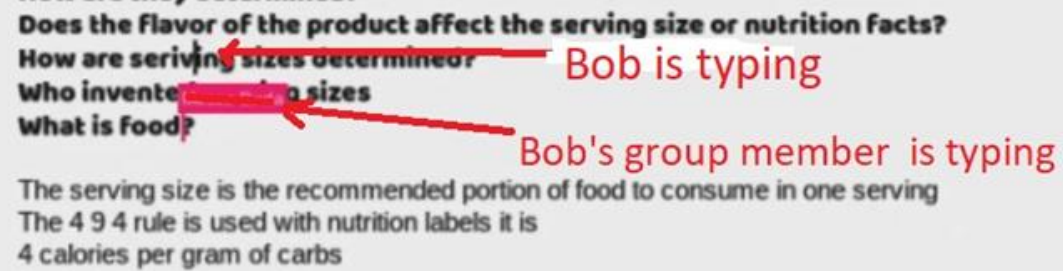

\section{Shaping Creating Collective Memories}

Tess believed in the importance of collaborative work. She encouraged her students to work collaboratively on shared documents. Thus, she justified that:

It [using the devices] seems to encourage some children to contribute, for example, to a shared doc. Where they might not if they're creating it with other people, they might be more motivated than if they have to create a whole piece of writing or a whole piece of something all by themselves.

She believed that collaborative work on the devices would encourage students to work. Her practices in the class reinforced this use:

I guess a lot of our teaching and demonstrations and resources that we presented to the kids, we were presenting on the interactive whiteboard or sharing Google docs with them, most of all that resources that were used were produced and presented digitally.

In addition, many tasks required using and working through shared documents and folders, such as folders that saved their leadership documents or scientific research documents. Tess said, "We've used Google docs and Google forms and all sorts of that kind of thing which allows easily for collaboration between children".

For this use, Google Drives and shared functions were essential. The students and the teachers in the school had accounts to create and share their information. Students had the skills to manage these accounts, as teachers taught them and provided guidelines for this purpose. The students worked collaboratively to create these collective memories. Tess linked students' collaboration skills and creating these memories: 
When we talked about some of the successful groups being the ones who have good leaders. I've talked about the kids choosing their own groups and how motivating that is. And I guess a way that the digital devices really can add, can add to enhance that is that particularly working on Google docs, they can all, like a group can all be working on the same document.

Student harmony was another factor that influenced this use. For example, Bob and one of her group members were working on the same document. Her friend was teasing her by changing what she typed, which created a small conflict that was sorted out quickly. 


\section{Connections Between Uses}

As in case one, there were connections between the ways of use. These connections are summarised in Table 5.1, below.

\section{Table 5.1}

Connections Between Uses in Case Two

\begin{tabular}{|c|c|c|c|c|c|}
\hline & $\begin{array}{c}\begin{array}{c}\text { Source of } \\
\text { information }\end{array} \\
\end{array}$ & & & & \\
\hline $\begin{array}{l}\text { Means of } \\
\text { communication }\end{array}$ & $\begin{array}{l}\text { Students } \\
\text { communicated } \\
\text { through the } \\
\text { devices to receive } \\
\text { or share } \\
\text { information }\end{array}$ & $\begin{array}{c}\text { Means of } \\
\text { communication }\end{array}$ & & & \\
\hline $\begin{array}{l}\text { Production } \\
\text { medium }\end{array}$ & $\begin{array}{l}\text { Students } \\
\text { searched for } \\
\text { information to } \\
\text { create their } \\
\text { digital and non- } \\
\text { digital products }\end{array}$ & $\begin{array}{l}\text { Students } \\
\text { communicated } \\
\text { through the } \\
\text { devices about their } \\
\text { products and } \\
\text { communicated } \\
\text { about using the } \\
\text { devices to create } \\
\text { their products }\end{array}$ & $\begin{array}{c}\text { Production } \\
\text { medium }\end{array}$ & & \\
\hline $\begin{array}{l}\text { External } \\
\text { personal } \\
\text { memory }\end{array}$ & $\begin{array}{l}\text { Students used } \\
\text { their personal } \\
\text { memories to find } \\
\text { information or } \\
\text { save information } \\
\text { they found }\end{array}$ & $\begin{array}{l}\text { Students } \\
\text { communicated } \\
\text { with teachers } \\
\text { through the } \\
\text { devices to receive } \\
\text { feedback on their } \\
\text { saved personal } \\
\text { work }\end{array}$ & $\begin{array}{l}\text { Students saved } \\
\text { and retrieved } \\
\text { their personal } \\
\text { digital } \\
\text { products }\end{array}$ & $\begin{array}{l}\text { External } \\
\text { personal } \\
\text { memory }\end{array}$ & \\
\hline $\begin{array}{l}\text { Collective } \\
\text { memory }\end{array}$ & $\begin{array}{l}\text { Students had } \\
\text { collective } \\
\text { memories } \\
\text { including } \\
\text { information } \\
\text { about class } \\
\text { activities }\end{array}$ & $\begin{array}{l}\text { Students } \\
\text { communicated } \\
\text { through the } \\
\text { devices to create } \\
\text { their collective } \\
\text { memories }\end{array}$ & $\begin{array}{l}\text { Students saved } \\
\text { and retrieved } \\
\text { their collective } \\
\text { digital } \\
\text { products }\end{array}$ & $\begin{array}{l}\text { No } \\
\text { evidence } \\
\text { found in } \\
\text { the study }\end{array}$ & $\begin{array}{c}\text { Collective } \\
\text { memory }\end{array}$ \\
\hline Research tool & $\begin{array}{l}\text { Students used } \\
\text { information they } \\
\text { found to design } \\
\text { their experiments }\end{array}$ & $\begin{array}{l}\text { Students } \\
\text { communicated } \\
\text { with participants } \\
\text { to collect data }\end{array}$ & $\begin{array}{l}\text { Students } \\
\text { produced } \\
\text { tables, photos, } \\
\text { and forms to } \\
\text { collect and } \\
\text { analyse data }\end{array}$ & $\begin{array}{l}\text { No } \\
\text { evidence } \\
\text { found in } \\
\text { the study }\end{array}$ & $\begin{array}{l}\text { Students } \\
\text { created, } \\
\text { saved, and } \\
\text { retrieved } \\
\text { forms and } \\
\text { tables for } \\
\text { research }\end{array}$ \\
\hline
\end{tabular}

\section{General Factors that Shaped All Uses}

As in case one, there were general factors that shaped all uses and factors that shaped particular ways of use. These factors were related to the educational system, school and classroom, teacher, students, and digital technologies. 
Tess witnessed the early integration of digital devices in classrooms, and explained how the development of digital technologies influenced using devices in general:

I can remember in about 1990 probably having a desktop computer that the children use[d], there were about six and ... they used something ... called Logo or something, which was just about giving the computer directions to make something happen. I never understood what it was. And it was so complicated to even log in or anything like that. But that was my very first experience.

In that time, Tess thought that using the devices was difficult and limited. She added, "I was not keen initially [to use the devices in the classroom] because I wasn't confident in using digital devices myself". That position changed 10 years later as digital technology functionality and ease of use developed. Alongside the changes in Tess's beliefs, there were general orientation and policies of New Zealand educational system to integrate digital technologies in teaching and learning and School B was no exception.

School B took an early decision to integrate digital technologies in its curriculum. Tess said:

The climate in the school was, not just in the school, obviously in society, that's the ... kids had to learn [using the devices] and so therefore I had to learn. So there was just a general feeling in the world. Also I guess the board and the school policy was, more and more devices were bought and put into classes and what's ever in the classes you have to use the application. And then I guess there was probably professional development that I had around how to incorporate digital devices and how to get value out of using them.

The school provided digital devices for their students; each classroom had several Chromebooks with a stable Internet connection. The school organised using devices in its venues; it was required that teachers and students sign an Internet Use Agreement and all classes did cyber safety training during the year. Each classroom had a projector and Chromecast to be used by the teachers and students. These policies enabled all ways of use to happen.

There were general factors related to the teacher; Tess's perspectives about learning and teaching developed significantly during her teaching career. She remembered her perspective 10 years ago: 
When I first started teaching, I probably would have told you that learning was reading and writing and maths, art and, those kinds of things, but they are very superficial level, not that kind of deeper developing your personality kind of thing.

This started to change when she and her colleagues have "become much more questioning about what constitutes learning". She explained her current perspectives by saying that learning involves "making your own understanding about the world and about life". She clarified "I guess it's making connections and making meaning in a way that enables you to become, a kind of, hopefully, an independent, successfully functioning adult in the world".

When I asked Tess about how she thinks students learn best, she addressed three qualities the environment should provide; firstly:

In an environment where they feel safe, by which, I mean accepted for who they are and valued for who they are and what they bring to their learning. Where they feel safe to take risks so they don't feel like they're going to be judged or shamed if they have got something wrong. Where they see the value of making mistakes.

Secondly, "where they're exposed to lots of opportunities. I think that's critical. Lots of different opportunities". Thirdly, "where they're getting specific feedback on what they're doing that supports them to move to the next level". According to Tess, acceptance, opportunities, and feedback were three essential qualities in the learning environment. One of the major areas that she and the other teachers worked on was "trying to encourage positive self-esteem and positive outlook on life and a positive attitude towards challenges. And we do quite a lot of specific teaching around how to manage those kinds of issues".

Tess acknowledged the influence of the professional development courses on her perspectives about learning: "I think I wouldn't have maybe the same deep perspective if it hadn't been for a lot of the professional development work that has gone on for me maybe in the last 10 years or so". Tess mentioned different workshop themes that influenced her teaching such as 21 st Century skills, inquiry learning, using technology in education, and thinking skills.

Tess emphasised that it was not the theoretical aspects per se in the workshops that impacted her perspectives about learning: 
Most of those different types of professional development that I was exposed to were based on someone's theory ... But ... I'm not academic. None of those really stick but what they did, I guess was they influenced someone who talked to me, and influence me in discussions with my colleagues, and then applying it in my classroom. I think that's what really developed my own understanding and taking those and having to try them out in my class.

This shows that the professional development activities that she participated in facilitated communicating with other teachers. She added:

I think the key thing is being encouraged to reflect on your own practice as a teacher and on the outcomes because it's looking at whether what you're doing is having an impact on students and whether they're learning. And if they're not, then you need to do something different.

This shows that providing theoretical stances, discussion with the colleagues, implementing the ideas in classrooms, and reflection on practice, all influenced her perspectives about learning.

One of the issues addressed in the workshops was " 21 st-century learning and about how that needs to be different" from Victorian education that focuses on reading and writing and maths. She said that there was a debate about "what education is really about and what learning is really about", especially "the changes that technology has wrought on the education system".

Tess believed in the importance of using digital devices in today's classrooms for two reasons. First, “It's just such a different world now, and they've just grown up with it as such an integral part of their lives". The second reason was, "I think it's really important in terms of motivation". This belief drove Tess to facilitate using the devices in students' learning.

At the same time, she was careful about the influence of using digital devices on students' learning. She said “I think it's [students' learning] deeper, but I don't know whether that's necessarily to do with the digital devices. I think that's about how we approach it because we're much more about looking at motivations and deep understandings". However, Tess thought that even the learning activities that do not necessarily require using digital devices still can benefit from using them. For example, for acting or physical education, "we may use a digital device to video the place so they can watch it back for practice or to time 
their training". She said, in general, "we still use the devices as a tool to enable whatever they're [the students] doing".

Tess believed in her students' capabilities of using digital technologies but still doubted hers: "I have very limited knowledge about what digital devices can do, and I know that they [her students] have far greater in different knowledge because of what they do which sometimes I don't understand at all'. She was aware that teachers' pedagogy could influence how the students would use the devices: "There may be ways that they could use the devices that they don't because they don't think they're allowed to". That belief explains the wide range of options she tried to give to her students.

Tess's beliefs, pedagogical knowledge, education, relationships, and experience worked together with the other factors when designing learning activities which shaped how students use devices and for what. Tess reported how students' capabilities influence using devices in general:

One of the things I find quite challenging is managing children's different levels of capability on digital devices. So it always seems like having children work together if they have different levels of ability is helpful. But what tends to happen is that the most able student takes over. So that's a constant struggle and it's probably a current issue is how we make that successful. In theory it is a really good idea to have more able supporting less able but we need to work out how to make that work better.

Findings reported in previous sections showed how this factor and other factors related to students shaped using digital devices for learning.

In conclusion, I borrow what Ian said in the interview:

What helped me to learn was definitely having access to the Chromebook and the Internet and just being able to hop on it and just type something up. And use Google docs and all these resources that they've given us to make presentations and put all that research together and stuff like that. And also our teachers helped a lot with helping refine our question and just generally making sure everything's acceptable.

What Ian said shows that all ways of use worked together with the other factors to make the learning happen. This make it hard to claim that outcomes can be attributed to a single factor in the classroom or that the factors can be separated from other factors. 


\section{Summary}

Six uses were addressed in case two: a source of information, a means of communication, a research tool, a production medium, an external personal memory, and a collective memory coordinator. As in case one, some factors shaped and enabled the six ways of use in general and some factors specifically influenced particular ways of use. The factors were classified in relation to the educational system, school and classroom environment, digital technologies, teachers, and students. The factors related to this case and case one are synthesised and outlined in the next chapter.

\section{The Contribution of Uses to Educational Purposes}

In this classroom, the two learning activities were designed to achieve different learning objectives. By analysing data collected from the documents and the interviews with Tess, the goals of the two activities were, first, to increase students' knowledge and understanding about the topics they have chosen, second, to undertake and understand a multi-phase process; and third, to work collaboratively. The goals aligned more with the qualification and socialisation functions of education. That does not mean that students' subjectification was not affected, but it means that the educational goals declared by the teacher focused more on the other functions. In the upcoming sections, I report how the six ways of use contributed to the educational purposes of the learning activities.

\section{Qualification}

The first and second goals align with the qualification function of education, which means providing knowledge and skills to do something, which was scientific research and leadership process.

\section{Increase Students' Knowledge and Understanding}

Increasing students' knowledge and understanding of science was a main requirement and objective of the scientific research learning activity, which aligns with the qualification function. Tess clarified, "Having a bit more of an understanding of what science is, being aware of the wide range of topics and subjects and ideas that come under the label of science". To achieve this objective Tess said, "we started right back at the beginning of this process doing a range of activities that were designed to help the kids explore different types of science like physics and biology and all the different aspects of science".

By the end of the term, the participants said that their knowledge and understanding of their topics increased. Bob said, "I learned about ... nutrition facts and serving sizes and 
now I have this habit of like whenever I see something ... Like ... how many calories did this have?" She compared her previous knowledge with her new knowledge:

Well, I didn't really know much about serving sizes. And I didn't realise actually how many discovers there were. And how many different kinds of science there is about it, finding caloric content. I don't know if I said that right. It's kind of interesting how much and my group didn't really know anything about.

Ian said, "I learned a lot about ... kinetic, friction, and magnetism but also about how to calculate those". He added, "Yeah it was very interesting and found ... lot about velocity ... It's very interesting and definitely worth the time". He then described the changes in his understanding of the meanings: "There's a lot of words that you think you know what they mean, but they mean something completely different". He gave an example: "there was the material world, that's why much of people got quite confused about it". Bob agreed with that and gave her personal experience of how the new knowledge influenced her understanding of science:

It's just some words I used in everyday context and then they completely changed when used in the meaning has completely changed when using a scientific context, like ... reflections, are one of those words. So because that's like reflection as in when you look into a mirror and reflection is in when you're reflecting back on yourself and like there's bunch of different meanings. There was some sciency ones, I can't remember.

Alice said that she learnt a lot about cameras. In her presentation, she wrote, "Together we found through our research that there are a lot of similarities between eyes and cameras basically there the same thing. Cameras are complicated to explain, but they are pretty easy to explain". She demonstrated her new knowledge by writing three articles explaining how the light moves and cameras work. During the observations, Alice used to read the information carefully from the webpage and summarise what she read on her logbook, which indicated that she did not just copy and paste what she found on the web.

On the other hand, Mitchel said that he did not learn anything. His response was expected, as he thought that school was not useful for him. In contrast, his presentation showed that he, at least, developed an understanding of some scientific concepts and used them, such as testing, hypothesis, conclusion, results, experiment, and reaction (Figure 5.21, 
below), considering that Mitchel was doing scientific research for the first time as he was in Year 7.

All the participants used the Internet as their main source of information. This was confirmed by Tess when she said:

I didn't see any of the groups for the library book. I think they did the research on the Internet, whatever research they managed, I can't remember the last time seeing the child use a library book for research. I keep trying, but they don't do it.

This was consistent with the observations. None of the participants' groups used another source of information except the Internet. Although the students used the library in the classroom for the daily reading activities and had regular visits to the school library, they only used the Internet to get information for their research projects. That provides evidence that using the devices as a source of information influenced how the students increased their knowledge and understanding.

\section{Figure 5.21}

Mitchel's Research Conclusion

\begin{tabular}{|l|}
\hline Conclusion: The results \\
of our experiment \\
showed that people had a \\
faster reaction time after \\
they had played a video \\
game for fifteen minutes. \\
This means that our \\
hypothesis was correct. \\
Next time I think we \\
could work faster and \\
stay focused better.
\end{tabular}

In addition to using the device as a source of information, other ways of use contributed to how the students knew more about their projects. Alice and Ian reported that they received emails from their peers that included information or links about their topics. They said this type of communication was helpful to find the information they were looking for. Collaboration to create collective memory in Bob's group involved adding answers from other members in her group to some questions in the shared document, which was used later 
to develop the final research questions. Bob described how using the device as a source of information and a personal memory helped her: "the website I'm using has an article on it, which means that the information is right there and copy it down and then put it in my own words later on". She also used her search history to find the information she was looking for.

On the other hand, having many sources of information may not suit all learners. As mentioned, the scientific research groups were divided into two cohorts: one cohort for the students who were doing their research for the second time, and a cohort for the students who were doing it for the first time. Tess said:

I think what I would do with them next time, I wouldn't let them all choose their own project. I think maybe we would work together as a whole class to come up with an idea for a science investigation and then we'd all do it together.

Tess justified that by limiting the options for the novice learners, she could support them more. In addition, they could focus on the basic knowledge and master it before doing their projects independently. Using the devices as a source of information had another side effect that the students reported, which was the distraction.

\section{Distraction}

Distraction was one of the themes that emerged in my analysis whenever the students mentioned using the devices as a source of information. Ian said, "Digital devices are great as long as you don't get distracted", which was agreed to by other participants. While using the digital devices for searching, the students reported that the main challenge was distraction. Ian said, "It's been very easy to get distracted, like sending you email. You reply, they reply you just keep on going back and forth or you find an interesting video". Mitchel gave an example by saying, "Yeah they [digital devices] distract me a lot because when I'm researching random stuff, like videos or something like people or music and stuff and then I kinda got in trouble". This was supported by the observations, as Mitchel was easily distracted while searching on the Internet. He tended to do random typing on his device or play games. Ian compared between getting information from the books and from the devices and said, "It is much easier [getting distracted] than searching things from the books". Tess confirmed that the main negative influence of digital devices on students' learning was related to "focusing, concentration, distraction". 


\section{Understanding and Undertaking Multiphase Projects}

Both scientific research and leadership activities were multi-phase projects. That required the students to go through different steps or phases to achieve the overall goal. This goal matches the qualification function. Tess said that the main overarching goal for the students in the science learning activity was, "to understand the scientific process and to know how to carry out a scientific experiment ... and ... for them to know and to work through all the different steps in that process. That's the big goal this year". Tess believed that for the students to be able to understand the scientific process they needed to do it:

That's quite a hard skill to teach and a hard skill to learn. And I think that would be something that they have had experience of before, but they still need lots more. Lots of them still find it incredibly difficult. So actually ... one of the things that ends up becoming a big focus of this is actually how to do that research.

The students showed different levels of understanding the scientific process, particularly the challenges of this process. Ian said, "I don't think any of my group really realised how much, how many calculations, how complicated magnetism really was". He added that it was not just the calculations that were challenging but the whole research process: "I don't think we really knew how much work and how complex it was going to be". Alice also reflected on the process of the scientific research when she was asked about her learning gains from this activity: "It's a lot harder to do things and come up with ideas that work for two people than for one", which points out handling the different perspectives within the scientific process.

Tess was satisfied with the outcomes of this learning activity with regards to understanding the scientific process:

I think I feel quite satisfied, that they do actually understand the scientific process and they overall they can design come up with a testable question and design a test that will collect measurable data and they will be able to draw a conclusion from the data. I feel satisfied about that.

I was able to follow up the scientific research from the beginning until the end, as the activity was conducted during the whole term. That was not the case with the leadership activity, as the activity ran through the whole year and faced distortions in the term that I was observing. Therefore, I was able to observe some phases of the leadership activity but not the whole project. 
Based on the themes that emerged from analysing the documents of the activities and the interviews with the teacher, I synthesised three milestones to achieve the goal of undertaking and understanding the multi-phase projects: developing the focus of the project; using and understanding the role of the data; and illustrating the outcomes of the project. The role of digital devices during this process is reported below.

\section{Developing a project focus:}

In scientific research, Tess said:

The research possibly could be a little bit challenging depending on what they chose, but you know, if I was working in a world without digital devices, then basically probably I would have maybe restricted their choices a little bit more. And maybe found a lot of library books and maybe limited to one area and maybe just controlled it a bit more by doing some background work about one topic and then ... your research or your questions need to be something to do with this topic.

Tess's point of view was that using digital devices gave the students more options and sources of information for "making choices themselves, having more control over what they're doing". The science fair of her classroom reflected this; there were diverse topics chosen by the students from different scientific areas. As an example, Bob said that she developed an interest in this topic because her mum follows a restricted diet, but searching the Internet made her take the decision:

My projects, about serving size, it's an experiment and officially the title is just the amount of food presented affect how much food you take, and I saw a video online and it kind of, it was about 100 calories actually looks like. And it kind of inspired me into serving sizes and things like that. And I asked my friends if they thought it was a good idea too.

Nevertheless, Tess reflected that for students who were doing the research project for the first time, providing many options could be unhelpful to master the core knowledge and provide adequate support for them:

It's really important and I think it's critical probably for those children [doing the project for the first time] to really get a lot out of the process, out of the lessons. I think they would have gotten that much more if either that was just one or maybe two projects and they could choose and each pair would still do it individually, do it 
themselves, but they'd be doing the same thing as the others, and we just step it through and ... control how they worked through it.

From Tess's point of view, it would be useful to provide a few options for beginners that the teacher and the students could manage, and when the students mastered the basic knowledge and skills, they could use them with other options. This indicates that the wide range of options provided by digital sources may not always be an advantage.

Developing research questions and a hypothesis was important to create a research focus. At the beginning of the term, Tess and the other teacher did different activities to demonstrate the concept of research questions as an integral part of scientific research:

[To] think of ideas and questions and then at the point that they were doing research, to know how to research something and being able to come up with good questions that lead you to come up with the information that you want.

Using the devices as a source of Information influenced participants' understanding of their research topic and the development of the research questions. Alice spent the first few weeks reading from the Internet and summarising the information until she was able to develop her focus. Bob started her research by forming many questions related to the serving sizes. Then she gathered information from different sources in one document to answer them, as reported in the ways of use section. Based on that initial research, Bob and her group were able to develop their main, focused research questions. Bob used different sources collected from the Internet to develop their focus on the topic. Bob said in the group interview, "I think I got better at phrasing the questions right. So Google would actually pick up what I wanted it to pick up" (Figure 5.22, below). Additionally, teachers used the collective memories, shared folders, so the students could retrieve and check the sources provided by the teachers. Bob and Ian were observed accessing them. 


\section{Figure 5.22}

Bob Developing Research Questions

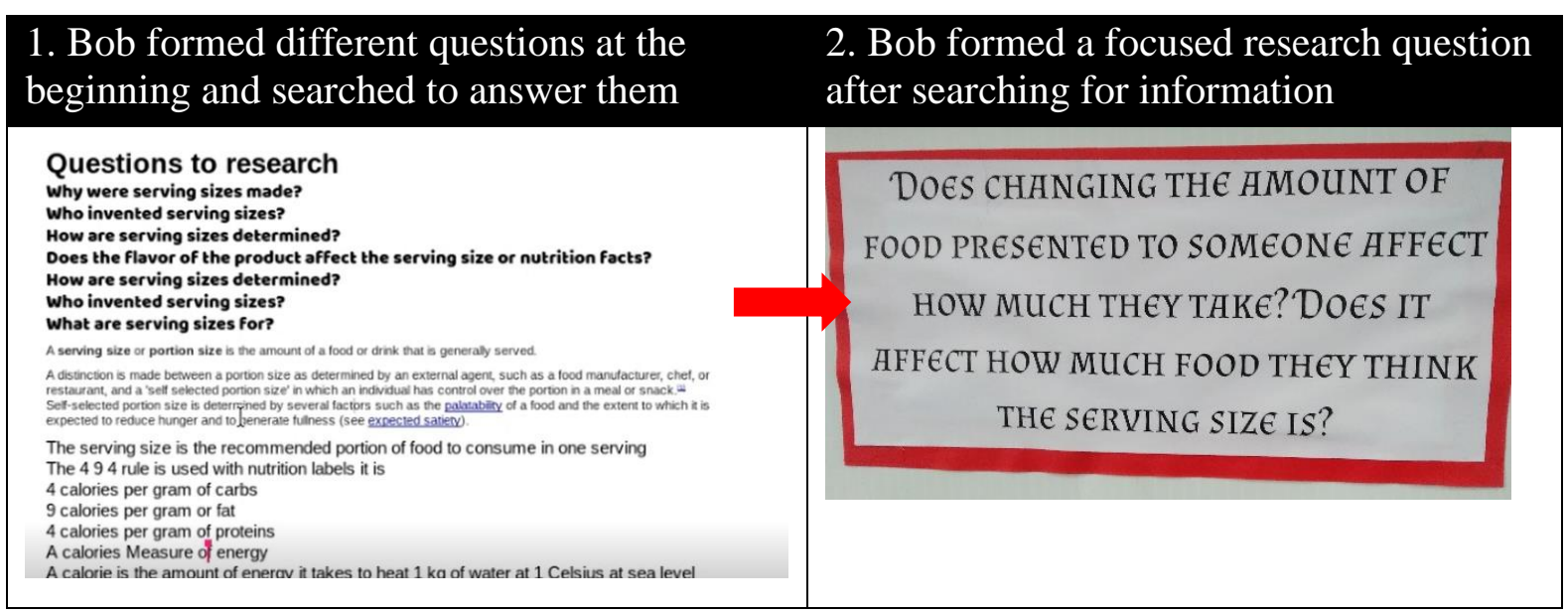

\section{Understanding the role of the data:}

Tess outlined the skills that were expected from the students to learn during scientific research that summarises the role of the data:

Fair testing! And learning how to make a fair test by repeating the tests lots of times to allow for anomalies and all those sorts of things. And then to be able to collect data and to analyse and present and to draw conclusions from the data. And to be able to come up with a final conclusion that says whether or not their hypothesis is supported or not.

The students needed to go through experimenting, collecting, and analysing data to provide evidence for their hypothesis. Using the devices as a research tool and as a source of information contributed to how some students learnt about the role of testing. Mitchel searched the Internet looking for evidence to prove his hypothesis, especially in YouTube. He found a procedure that could be used to test his hypothesis and then used the devices for that. Interestingly, when he was asked about what things he learnt from this learning activity he first said "nothing". Then he said that he wanted to prove that gaming was good for his reaction time and was able to prove that by searching and testing:

I'm pretty sure it [gaming] does help. I think I'm pretty sure it is ... it does make your reaction time faster because I researched it and then I looked through all these things and it said that it didn't know, But then Tess said, well you have to keep on trying. You have to keep on seeing if it's true or not when you're testing. 
It was not clear if Mitchel was not aware of his learning gains or he did not want to admit that school was useful for him, as he had a negative attitude towards school. Actually, he used scientific logic to some extent. Tess said:

A lot of the children in my class, Mitchel included, were determined that the testing was going to be about video games and it started because they wanted to prove that playing "Fortnite" was good for them. So it was good in that it gave them kind of a real world idea for their testing. You can't argue with that. That's what they were doing.

On the other hand, Tess was aware of the side effects realised from the superficial understanding of scientific methods:

But it was bad because they were really fixated on how they could set up their experiment so that it gave them the excuse to play "Fortnite" at school. Basically, it's what they were trying to come up with. So that was just an interesting little side I'm not excited about. And I mean the great thing was that it showed that they really understood the purpose of the whole thing. I mean the purpose of the whole thing is to come up with a hypothesis and try and prove it.

Additionally, the students in Tess's classroom used the devices as a research tool when they faced difficulties in their testing, such as the group who used it to measure the height of the bounce. Hence, devices provided more options and ways for experimentation.

Collecting data in the leadership learning activity helped Bob to realise the role of the data in decision making. While working she said, "We sent out the Google forms ... we have been collecting the gear and then working out how much each class is going to have". By the end of the term she emphasised these gains by saying:

We learnt how to make Google forms. That was really awesome, and we learnt about all the different kinds of questions ... Then we realised it would actually be a good idea if we redistributed the PE gear. So we're sending out forms and our goal is to redistribute $\mathrm{PE}$ gear.

While collecting data can happen in different ways, using devices for this purpose provided an easy and quick way in the learning activity. 


\section{Illustrating Knowledge}

Tess considered presenting students' learning as part of the learning process. The students presented their learning in two ways: by using the display board as shown and by creating models. Ian said, "I learned quite a bit about how to phrase things that in the order which you need to go to present your research". Ian and Alice designed models of their work. Using the devices as a source of information contributed to illustrate their learning. Ian designed with his group a model of the maglev train; they researched four designs before they decided to adopt one (Figure 5.23, below). He said, "I think I'm more willing to just try things out, and not really minding how it turns out". He described how his group chose their model of the magnetic train, “we'll just try this and if it doesn't work well we just kept on trying and so I think I was more open to trying things".

\section{Figure 5.23}

Using Devices as a Source of Information to Create Models Illustrating Learning

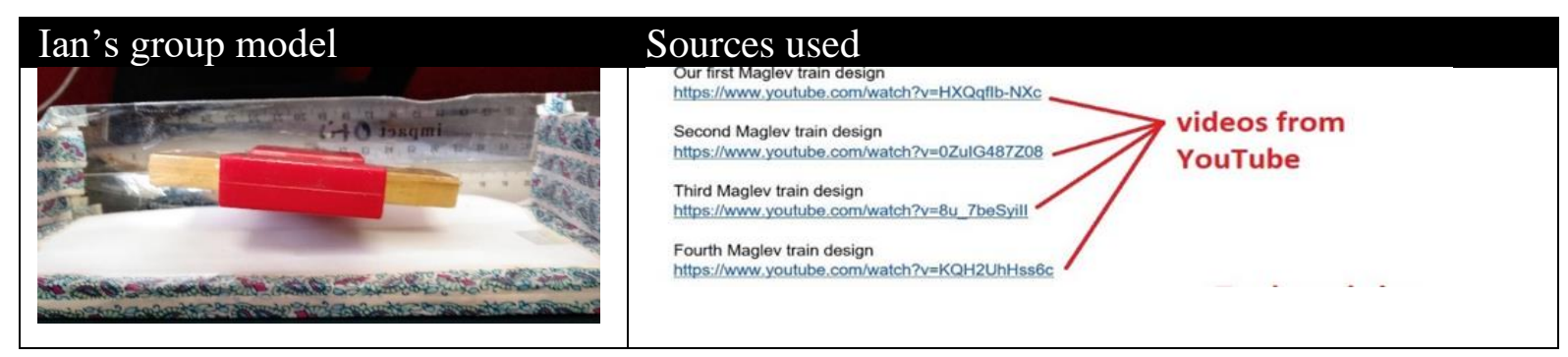

Alice and her friend designed a model that illustrated their learning. They used devices as a source of information to design a camera obscura the other students in the classroom can enter and see how the light travels through the camera (Figure 5.24, below).

\section{Figure 5.24}

\section{Alice's Group Model}

\section{Alice's group model}

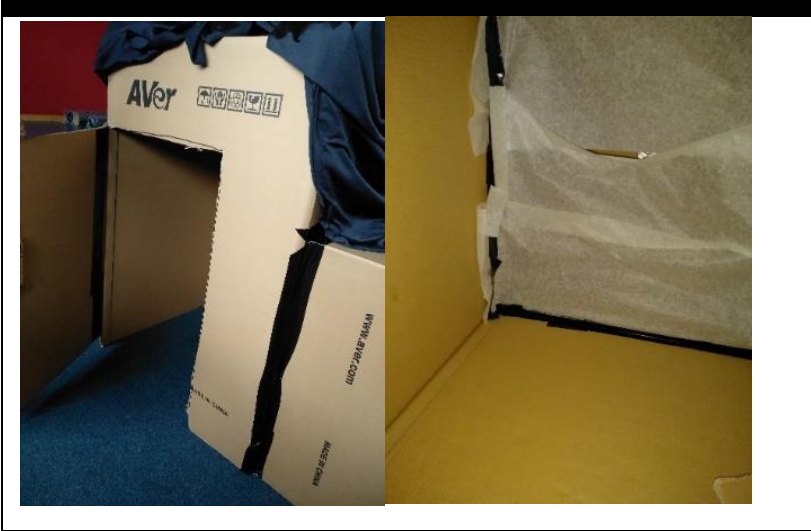


Tess was aware of the potential provided by digital devices to produce things that illustrate students' learning in the digital era compared to the past:

My initial thoughts are kind of in terms of the outcomes, what the kids are producing now ... I think one of the big differences would probably be the variety of what we do, how we learn and the variety of ways that students present their learning. So I think back then most information that came to the kids was via books or I guess real life experiences, but mostly through books. Occasionally there would be something like maybe a movie, a video I guess in those days, but not very often at all or maybe listening to something. Whereas now a lot of the motivation and the stimulus that they see as clips on YouTube or its digital photos ... that's probably the biggest difference.

These findings show that the six ways of use, in this case, contributed to students' qualifications, which supports the findings from case one.

\section{Socialisation}

The third goal, to work collaboratively, was consistent with socialisation function of education, as it contributes to students' membership in their community.

\section{Working Collaboratively}

The students in Tess's class were required to work collaboratively to do both the leadership activity and the scientific research activity. Tess said that one of the main reasons to use a digital device was to facilitate collaborative work:

The collaborative aspect of using digital devices because actually that's, I guess one of the key things that underpin a lot of why we use digital devices in schools. I think we believe that there is a great deal of power and kids working collaboratively.

Collaborative work required different intertwined ways of use to be accomplished. Tess gave an example of how using the devices helped students collaborative work: "Communication, and producing the stuff like the Google forms, making plans on google docs and sharing them with other people. Getting people to sign up for things via google docs. and All those kinds of things". Tess highlighted different uses interconnected together during collaborative work: a means of communication, a collective memory, and a production medium. 
Tess said that one of the benefits of using the devices for collaborative work was "Being able to organise themselves independently ... Ian's group did a lot of work outside school". Ian supported that: "To complete on time, we needed to do a lot of work outside of school. Having Google docs mean that we could collaborate with each other outside of school and be able to communicate and complete work". He added, "I think we managed to do a lot of work in that short amount of time that we had". In the leadership activity, Alice reported the same. She said that the devices were helpful for them as a group to communicate together and with people from outside, which facilitated their cooperation and reduced the time needed to do their tasks. She reported that one of the main things she learnt from this activity was "how to cooperate, mostly, and how to do something in a very short amount of time".

Communication through the devices helped the students in planning and making early decisions. Tess said, "all the planning and all that kind of thing they've done on the Chromebooks". When I asked Ian what he learnt while doing leadership activity, he replied, "I think how to plan, planning to events. Learning how to do that. And how to get confirmation from teachers or check if other events are gonna get in the way". Ian used the devices to communicate with teachers for the events. The collective memories created by the students facilitated the collaborative work; Ian, Bob, and Alice used their shared drives and documents to do their tasks such as preparing the articles for their demonstrations and checking each other's contributions.

This shows that digital devices played a significant role with regards to collaborative work, which was important to achieve the learning goals of the learning activities.

\section{Summary}

The findings of the second case study showed that using devices contributed to the qualification function of education. Using devices as production medium, collective memory coordinator, and a means of communication contributed to the socialisation function of education, which is consistent with the findings from the first case study. The next chapter summarises and synthesises the findings from the two classrooms to answer the research questions. 


\section{Chapter 6 Synthesis of Research Findings}

This study aimed to answer the following question: How do students use digital devices for educational purposes in primary classrooms? To answer this question, three sub-questions were addressed:

1. In what ways do primary students use their digital devices in classroom learning activities?

2. What factors shape the ways of use in classrooms?

3. How do the ways of use contribute to the educational purposes of classroom learning activities?

The next sections summarise and synthesise the findings of the case studies to answer the research questions.

\section{In What Ways Do Primary Students Use Their Digital Devices in Classroom Learning Activities?}

Six ways of use and the connections between them were identified in each case, as illustrated in Figure 6.1, below. Five uses were identified in the two cases and two differentiated. The five similar uses were: (1) source of information, (2) means of communication, (3) production medium, (4) external personal memory, and (5) collective memory coordinator. There was one way of use specific to each case: for case one, using devices as a trial-and-error learning space and for case two, using devices as a research tool. The uses and the connections of the two cases are synthesised in Table 6.1 and Table 6.2, below.

The ways of use were not isolated; the students engaged more than one use to do their tasks. Although the uses had the same general characteristics in both settings, the students' use varied. For example, participants in both classes used the devices as a production medium, but one of the classes produced only digital artefacts and the other created digital and non-digital artefacts. Also, using the devices as a source of information was intensive and extensive in one of the classes while it was limited and less frequent in the other one. 


\section{Figure 6.1}

The Ways of Use in Each Case

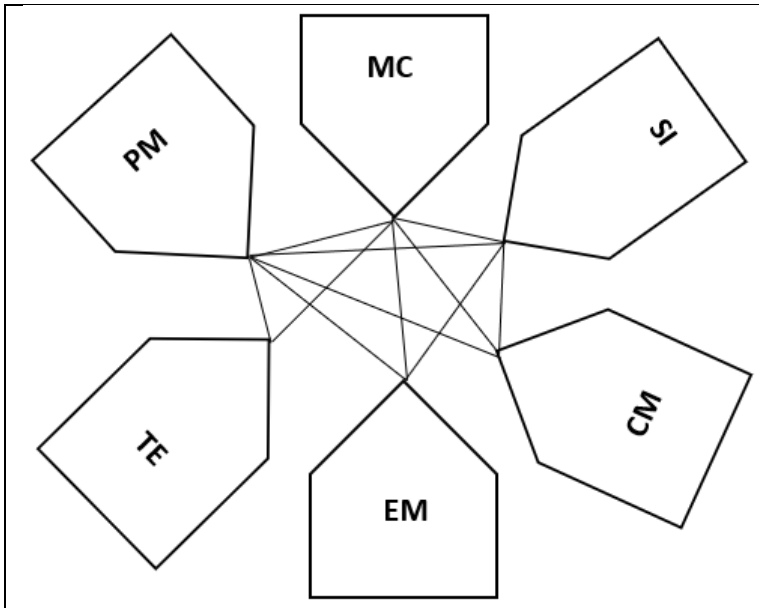

Case one

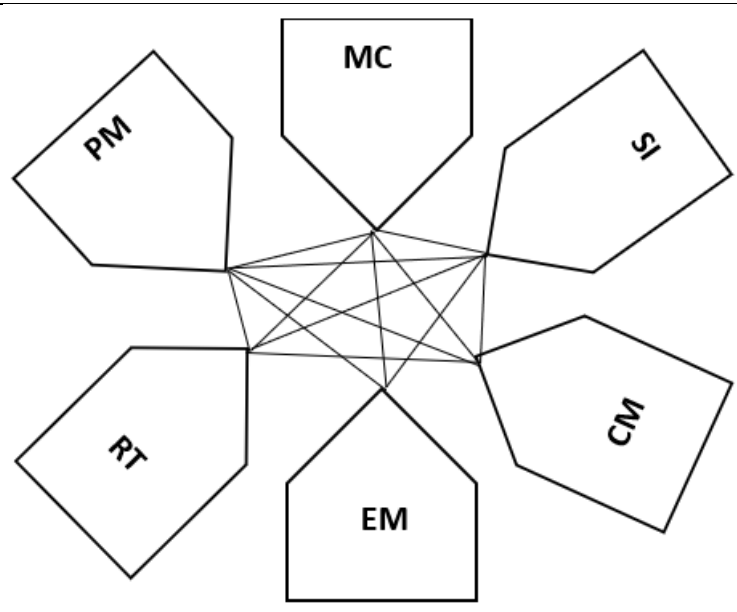

Case two

Note: SI: Source of information. MC: Means of communication. PM: Production medium. RT: Research tool.

EM: External personal memory. CM: Collective memory coordinator. TE: Trial-and-error learning space.

Table 6.1

A Summary and Synthesis of Connections Between Uses of the Two Cases

\begin{tabular}{|c|c|c|c|c|c|}
\hline & $\begin{array}{c}\text { Source of } \\
\text { information }\end{array}$ & & & & \\
\hline $\begin{array}{l}\text { Means of } \\
\text { communication }\end{array}$ & $\checkmark$ & $\begin{array}{c}\text { Means of } \\
\text { communication }\end{array}$ & & & \\
\hline $\begin{array}{l}\text { Production } \\
\text { medium }\end{array}$ & $\checkmark$ & $\checkmark$ & $\begin{array}{l}\text { Production } \\
\text { medium }\end{array}$ & & \\
\hline $\begin{array}{l}\text { Personal } \\
\text { memory }\end{array}$ & $\checkmark$ & $\checkmark$ & $\checkmark$ & $\begin{array}{l}\text { Personal } \\
\text { memory }\end{array}$ & \\
\hline $\begin{array}{l}\text { Collective } \\
\text { memory }\end{array}$ & $\checkmark$ & $\checkmark$ & $\checkmark$ & $x$ & $\begin{array}{l}\text { Collective } \\
\text { memory }\end{array}$ \\
\hline $\begin{array}{l}\text { Trial-and- } \\
\text { error learning } \\
\text { space }\end{array}$ & $x$ & $\checkmark$ & $\checkmark$ & $x$ & $x$ \\
\hline Research tool & $\checkmark$ & $\checkmark$ & $\checkmark$ & $x$ & $\checkmark$ \\
\hline$\checkmark$ & Evidence was & ound in this study & & & \\
\hline$x$ & No evidence $v$ & as found in this stu & & & \\
\hline
\end{tabular}


Table 6.2

Identification of the Ways of Use of Digital Devices for Classroom Learning

\begin{tabular}{|c|c|}
\hline The use & A description of the use \\
\hline $\begin{array}{l}\text { Source of } \\
\text { information: }\end{array}$ & $\begin{array}{l}\text { * Passive use: students receive information by accessing links or } \\
\text { documents shared by others through emails or shared drives. } \\
\text { * Interactive use: students search for information using search } \\
\text { engines. They use keywords, questions, or history to locate } \\
\text { information from websites, Wikis, YouTube, and journals. } \\
\text { * Students deal with different forms of resources: text, audio, } \\
\text { video, and images. }\end{array}$ \\
\hline $\begin{array}{l}\text { Means of } \\
\text { communication }\end{array}$ & $\begin{array}{l}\text { * Students use devices to communicate about devices with peers } \\
\text { and teachers as a main topic: } \\
\text { - to exchange expertise about using the devices. } \\
\text { - to reflect on learning while using the devices. } \\
\text { * Students use devices to communicate through devices with } \\
\text { teachers, peers, family members, and people from outside school: } \\
\text { - to exchange feedback } \\
\text { - to organise group work } \\
\text { - for inquiries. }\end{array}$ \\
\hline Production medium & $\begin{array}{l}\text { * Students use devices to create digital and non-digital artefacts } \\
\text { individually and collaboratively. } \\
\text { * Students create digital products in different formats such as: text, } \\
\text { 2D and 3D models, and music. } \\
\text { * Students illustrate their learning through their products. }\end{array}$ \\
\hline $\begin{array}{l}\text { External personal } \\
\text { memory }\end{array}$ & $\begin{array}{l}\text { * Students use devices to create personal accounts to save and } \\
\text { retrieve digital artefacts, collected information, and history. } \\
\text { * Students control who can access their personal accounts. }\end{array}$ \\
\hline $\begin{array}{l}\text { Collective memory } \\
\text { coordinator }\end{array}$ & $\begin{array}{l}\text { * Students collaboratively use devices to create, save, and retrieve } \\
\text { digital artefacts and collected information. } \\
\text { * Students have shared access to their shared spaces. } \\
* \text { This use is correlative with communication and production uses. }\end{array}$ \\
\hline $\begin{array}{l}\text { Trial-and-error } \\
\text { learning space }\end{array}$ & $\begin{array}{l}\text { * Students use devices to explore and try application features to } \\
\text { master digital skills. } \\
\text { * Students test ideas, designs, or models to create their digital } \\
\text { products. }\end{array}$ \\
\hline Research tool & $\begin{array}{l}\text { * Students use devices to collect data through online surveys and/or } \\
\text { record data by direct data entry. } \\
\text { * Student use the devices for testing and experimentation. } \\
\text { * Students use the devices to analyse data by using spreadsheets, } \\
\text { tables, and graphs. }\end{array}$ \\
\hline
\end{tabular}




\section{What Factors Shape the Ways of Use in Classrooms?}

Intertwined factors enabled and shaped the seven ways; they were classified in relation to the interacting elements in the classroom setting, which were educational system, school system and classrooms, digital technologies, teachers, and students. Figure 6.2, below, illustrates these factors. The intersected circles were used to emphasise the interconnectedness of the factors. Tables 6.3-6.10 summarise and synthesise the factors extracted from the two cases that shaped the ways of use. There were general factors that enabled and shaped all uses. For example, the flexible curriculum of the educational system allowed the schools and teachers to choose their approach in integrating digital devices. Another example is the affordances of the Web 2.0 technologies; without these technologies probably most of the uses described would not be possible. On the other hand, there were factors that clearly shaped specific ways of use but were not necessarily related to other uses. For example, teachers' beliefs about the importance of collaborative learning were related to using the devices as a means of communication and collective memory, but it was not found related to using the device as a personal memory. The findings gave examples of how these factors worked synergistically to enable and shape the uses. Additionally, the findings showed how the factors influenced each other alongside shaping the uses.

\section{Figure 6.2}

Interconnected Factors Shaping Using Devices in Classroom Settings

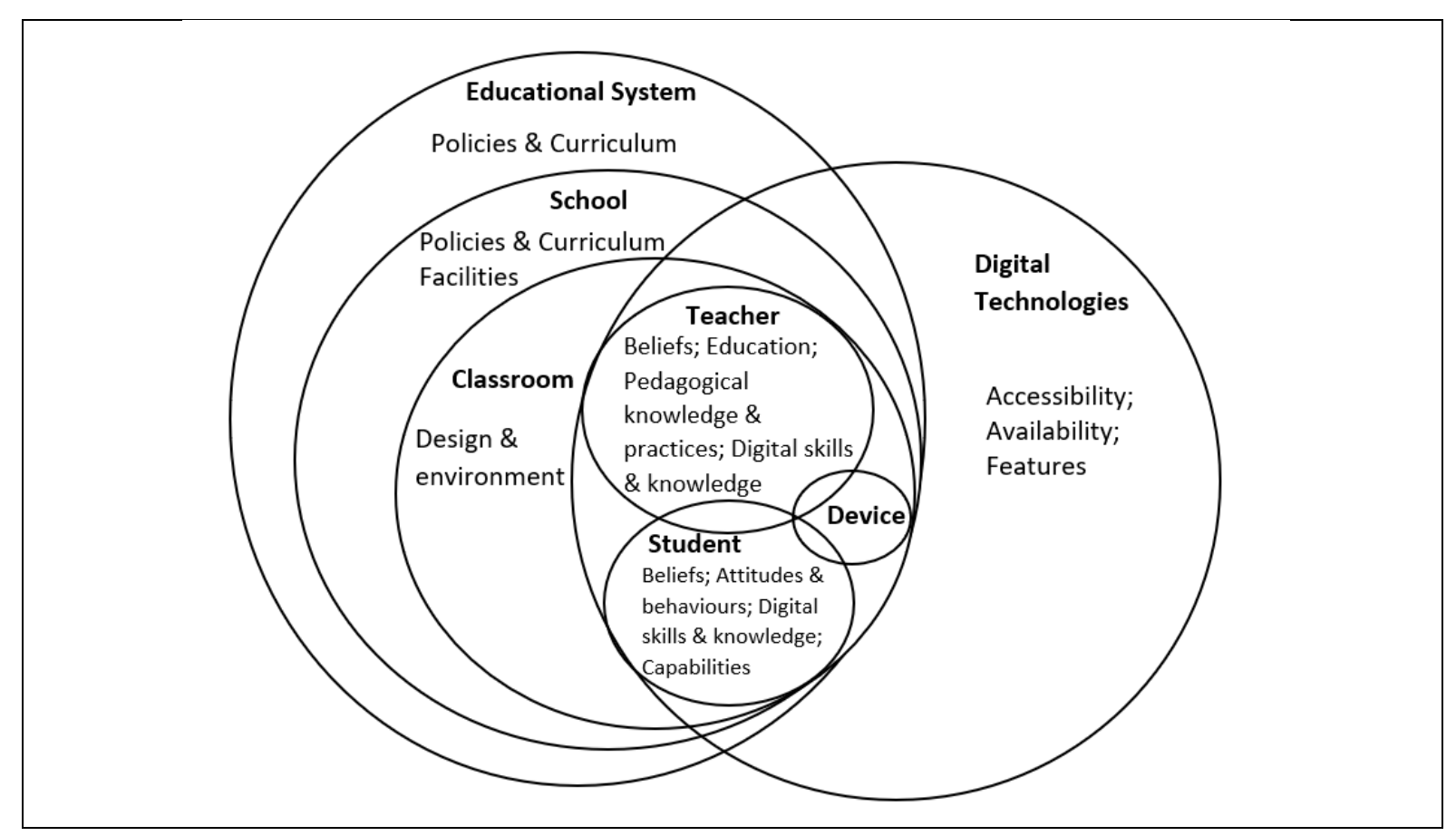


Table 6.3

General Factors that Shaped Using Digital Devices in Classrooms

\begin{tabular}{|c|c|c|}
\hline Element & Factors & All Ways of Use \\
\hline $\begin{array}{l}\text { Educational } \\
\text { system }\end{array}$ & $\begin{array}{l}\text { Policies \& } \\
\text { curriculum }\end{array}$ & $\begin{array}{l}\text { Flexibility and curriculum that promote the agency to } \\
\text { schools, teachers, and students to use the devices in } \\
\text { different ways and for different purposes. }\end{array}$ \\
\hline \multirow[t]{2}{*}{$\begin{array}{l}\text { School \& } \\
\text { classrooms }\end{array}$} & $\begin{array}{l}\text { Policies \& } \\
\text { curriculum }\end{array}$ & $\begin{array}{l}\text { School policies for integrating digital technologies: social } \\
\text { justice position, BYOD or providing devices for each } \\
\text { student policies, teachers' professional development } \\
\text { training, school curriculum. Clear school policy of how to } \\
\text { use the devices in a form of agreements, e.g. 'Kawa of } \\
\text { care'. Classroom designs that allow students' interactions. }\end{array}$ \\
\hline & Facilities & $\begin{array}{l}\text { Providing stable and fast Internet access, chargers, and } \\
\text { storage spaces for devices, and technical support to the } \\
\text { students and the staff. }\end{array}$ \\
\hline \multirow[t]{4}{*}{ Teachers } & Beliefs & $\begin{array}{l}\text { Digital devices are useful for learning and could enhance } \\
\text { students' learning. Digital devices provide opportunities } \\
\text { and control over learning. Using devices motivates } \\
\text { students. Students' interaction with digital technologies is } \\
\text { essential for } 21 \text { st century. }\end{array}$ \\
\hline & Education & $\begin{array}{l}\text { Pre-service education and in-service professional } \\
\text { development experiences. }\end{array}$ \\
\hline & $\begin{array}{l}\text { Pedagogical } \\
\text { knowledge \& } \\
\text { practices }\end{array}$ & $\begin{array}{l}\text { Pedagogical choices related to nature and the goals of the } \\
\text { learning activities. Theoretical choices about using and } \\
\text { integrating digital devices in teaching: SAMR model, } \\
21 \text { st century Skills. Knowledge about their students and } \\
\text { context. Providing clear goals for the purposes of using } \\
\text { devices. Collaboration with teachers. }\end{array}$ \\
\hline & $\begin{array}{l}\text { Digital skills } \\
\& \text { knowledge }\end{array}$ & Digital skills and knowledge about digital technologies. \\
\hline \multirow[t]{3}{*}{ Students } & Beliefs & Chromebooks and the Internet are helpful for learning. \\
\hline & $\begin{array}{l}\text { Attitudes \& } \\
\text { behaviours }\end{array}$ & $\begin{array}{l}\text { Attitudes towards school learning, commitment to school } \\
\text { policies related to using devices. }\end{array}$ \\
\hline & $\begin{array}{l}\text { Digital skills } \\
\& \text { knowledge }\end{array}$ & Capabilities of using devices and the Internet. \\
\hline Technology & $\begin{array}{l}\text { Accessibility } \\
\& \text { availability }\end{array}$ & $\begin{array}{l}\text { Available Web } 2.0 \text { technologies and being able to access } \\
\text { them. }\end{array}$ \\
\hline
\end{tabular}


Table 6.4

Factors that Shaped Using Devices as a Source of Information

\begin{tabular}{|c|c|c|}
\hline Element & Factors & Source of Information \\
\hline \multirow[t]{3}{*}{ Teachers } & Beliefs & $\begin{array}{l}\text { Digital devices provide unique and varied sources of } \\
\text { information. Students prefer digital sources. }\end{array}$ \\
\hline & $\begin{array}{l}\text { Pedagogical } \\
\text { knowledge \& } \\
\text { practices }\end{array}$ & $\begin{array}{l}\text { Using digital sources with the students in the class. } \\
\text { Providing instructions for searching and locating } \\
\text { information from the Internet. }\end{array}$ \\
\hline & $\begin{array}{l}\text { Digital skills \& } \\
\text { knowledge }\end{array}$ & Knowledge of digital sources. \\
\hline \multirow[t]{4}{*}{ Students } & Beliefs & $\begin{array}{l}\text { Digital devices are a main source of information, which } \\
\text { is rich, fast, and easy to use. }\end{array}$ \\
\hline & $\begin{array}{l}\text { Attitudes \& } \\
\text { behaviours }\end{array}$ & $\begin{array}{l}\text { Preference for using digital sources (text, video, etc.). } \\
\text { Following/using teachers' instructions about locating } \\
\text { information. Self-control related to managing distraction }\end{array}$ \\
\hline & $\begin{array}{l}\text { Digital skills \& } \\
\text { knowledge }\end{array}$ & Using and locating digital sources. \\
\hline & Capabilities & Reading. \\
\hline \multirow[t]{2}{*}{ Technology } & Availability & Searching engines. Online sources. \\
\hline & Features & $\begin{array}{l}\text { Hyperlinks, pop-up bars, auto-correct for spelling, and } \\
\text { sharing feature. }\end{array}$ \\
\hline
\end{tabular}

\section{Table 6.5}

Factors that Shaped Using Devices as a Means of Communication

\begin{tabular}{|c|c|c|}
\hline Element & Factors & Means of Communication \\
\hline \multirow[t]{2}{*}{ Teachers } & Beliefs & $\begin{array}{l}\text { Students learn better when communicating with others. } \\
\text { Devices offer unique ways for communicating with } \\
\text { others. Digital technologies are efficient for feedback. }\end{array}$ \\
\hline & $\begin{array}{l}\text { Pedagogical } \\
\text { knowledge \& } \\
\text { practices }\end{array}$ & $\begin{array}{l}\text { Using mixed-ability groups and collaborative problem- } \\
\text { solving strategies. Facilitating communication about the } \\
\text { devices in class activities. Giving and encouraging giving } \\
\text { feedback through the devices. Giving instructions and } \\
\text { lessons about communication through the devices. }\end{array}$ \\
\hline \multirow[t]{3}{*}{ Students } & Beliefs & Communicating with peers about digital skills is useful. \\
\hline & $\begin{array}{l}\text { Attitudes \& } \\
\text { behaviours }\end{array}$ & $\begin{array}{l}\text { Attitude towards collaborative work including } \\
\text { interactions, relationships, and collaboration. Using } \\
\text { devices for feedback. Self-esteem related to one's own } \\
\text { digital skills and confidence to share ideas. }\end{array}$ \\
\hline & $\begin{array}{l}\text { Digital skills \& } \\
\text { knowledge }\end{array}$ & $\begin{array}{l}\text { Using applications for communication to give feedback, } \\
\text { send emails, and text messaging. }\end{array}$ \\
\hline \multirow[t]{2}{*}{ Technology } & Availability & $\begin{array}{l}\text { Applications for communication: Emails, Google drive, } \\
\text { text messaging apps, Skype. }\end{array}$ \\
\hline & Features & $\begin{array}{l}\text { Sharing and adding comments in blogs and Google } \\
\text { drives. }\end{array}$ \\
\hline $\begin{array}{l}\text { School \& } \\
\text { classrooms }\end{array}$ & $\begin{array}{l}\text { Classroom } \\
\text { environment }\end{array}$ & $\begin{array}{l}\text { Classroom design that allowed students to sit in groups } \\
\text { and communicate about devices }\end{array}$ \\
\hline
\end{tabular}


Table 6.6

Factors that Shaped Using Devices as a Production Medium

\begin{tabular}{|c|c|c|}
\hline Element & Factors & Production Medium \\
\hline \multirow[t]{2}{*}{ Teachers } & Beliefs & $\begin{array}{l}\text { Digital devices enable students to illustrate their learning } \\
\text { in different ways. Using devices for production is one of } \\
\text { the options. }\end{array}$ \\
\hline & $\begin{array}{l}\text { Pedagogical } \\
\text { knowledge \& } \\
\text { practices }\end{array}$ & $\begin{array}{l}\text { Engaging students' culture. Providing a framework for } \\
\text { the production process and engaging students in } \\
\text { developing success criteria. Collaboration with teachers. } \\
\text { Teaching strategies: detailed instructions, collaborative } \\
\text { work. Using samples, tutorials, and templates with guided } \\
\text { questions. Supervising collaborative products. Time } \\
\text { management. }\end{array}$ \\
\hline \multirow[t]{3}{*}{ Students } & $\begin{array}{l}\text { Attitudes \& } \\
\text { behaviours }\end{array}$ & $\begin{array}{l}\text { Preferences and interests, relationships, and attitudes } \\
\text { towards group work. }\end{array}$ \\
\hline & $\begin{array}{l}\text { Digital skills \& } \\
\text { knowledge }\end{array}$ & $\begin{array}{l}\text { Skills and knowledge about application features and } \\
\text { potentials, and typing skills. Prior knowledge and } \\
\text { experience. }\end{array}$ \\
\hline & Capabilities & Reading and typing. \\
\hline \multirow[t]{2}{*}{ Technology } & Availability & $\begin{array}{l}\text { Applications for different purposes: word processing, } \\
\text { presentation, music, 3D design, and coding software. }\end{array}$ \\
\hline & Features & $\begin{array}{l}\text { Editing tools, photo-editing tools, spell check, sound } \\
\text { recording, and transcribers. }\end{array}$ \\
\hline $\begin{array}{l}\text { Schools \& } \\
\text { classrooms }\end{array}$ & $\begin{array}{l}\text { Classroom } \\
\text { environment }\end{array}$ & $\begin{array}{l}\text { Classroom design that allows students to sit in groups and } \\
\text { communicate about devices. }\end{array}$ \\
\hline
\end{tabular}

Table 6.7

Factors that Shaped Using Devices as a Personal Memory

\begin{tabular}{lll}
\hline Element & Factors & Personal Memory \\
\hline Teachers & Beliefs & Students find it useful to save their work on the devices. \\
\cline { 2 - 3 } & $\begin{array}{l}\text { Pedagogical } \\
\text { knowledge \& } \\
\text { practices }\end{array}$ & $\begin{array}{l}\text { Structured teaching and detailed instructions about } \\
\text { managing personal accounts. Saving and retrieving are } \\
\text { part of the learning activities. Teaching strategies such as } \\
\text { learning stories and Cool Quick Write. }\end{array}$ \\
\hline Students & $\begin{array}{l}\text { Digital skills } \\
\text { \& knowledge }\end{array}$ & $\begin{array}{l}\text { Saving, retrieving, and managing personal accounts and } \\
\text { history; using sharing and privacy features. }\end{array}$ \\
\hline Technology & Availability & Personal accounts in drives and applications \\
\cline { 2 - 3 } & Features & $\begin{array}{l}\text { Passwords, storage limit, saved history, and privacy and } \\
\text { sharing features. }\end{array}$ \\
\hline
\end{tabular}


Table 6.8

Factors that Shaped Using Devices as a Collective Memory Coordinator

\begin{tabular}{lll}
\hline Element & Factors & Collective Memory \\
\hline Teachers & Beliefs & Collaborative learning is important for students' learning. \\
\cline { 2 - 3 } & $\begin{array}{l}\text { Pedagogical } \\
\text { knowledge \& } \\
\text { practices }\end{array}$ & $\begin{array}{l}\text { Using collective memories with the students. Teaching } \\
\text { strategies: mixed groups, direct instructions. }\end{array}$ \\
\hline Students & $\begin{array}{l}\text { Attitudes \& } \\
\text { behaviours }\end{array}$ & Collaboration arrangements, interactions, and norms. \\
\cline { 2 - 3 } & $\begin{array}{l}\text { Digital skills } \\
\& \text { knowledge }\end{array}$ & $\begin{array}{l}\text { Creating, accessing, and using shared documents and } \\
\text { folders. }\end{array}$ \\
\hline Technology & Availability & Shared drives \& folders. \\
\cline { 2 - 4 } & Features & $\begin{array}{l}\text { Simultaneous access. } \\
\text { Automatic saving and sharing features. }\end{array}$ \\
& &
\end{tabular}

Table 6.9

Factors that Shaped Using Devices as a Trial-and-Error Learning Space

\begin{tabular}{lll}
\hline Element & Factors & Trial-and-Error Learning \\
\hline Teachers & $\begin{array}{l}\text { Pedagogical } \\
\text { knowledge \& } \\
\text { practices }\end{array}$ & $\begin{array}{l}\text { Choosing activities that required developing digital skills. } \\
\text { Providing opportunities, time, and encouragement to } \\
\text { explore and make mistakes while learning. Explicit } \\
\text { instructions to explore, take risks, and try things while } \\
\text { working on the devices. }\end{array}$ \\
\hline Students & $\begin{array}{l}\text { Attitudes \& } \\
\text { behaviours }\end{array}$ & Taking risks and trying new things. \\
\cline { 2 - 3 } & $\begin{array}{l}\text { Digital skills } \\
\& \text { knowledge }\end{array}$ & Using features and functions of the application. \\
\hline Technology & Features & Modification features, especially 'undo' function. \\
\hline
\end{tabular}

Table 6.10

Factors that Shaped Using Devices as a Research Tool

\begin{tabular}{|c|c|c|}
\hline Element & Factors & Research Tool \\
\hline Teachers & $\begin{array}{l}\text { Pedagogical } \\
\text { knowledge \& } \\
\text { practices }\end{array}$ & $\begin{array}{l}\text { Choosing activities that require using devices as research } \\
\text { tools. } \\
\text { Teaching and instructions about how to use the devices to } \\
\text { collect and analyse data. }\end{array}$ \\
\hline \multirow[t]{2}{*}{ Students } & $\begin{array}{l}\text { Digital skills } \\
\& \text { knowledge }\end{array}$ & Using spreadsheets, photo editing, and collecting data. \\
\hline & Capabilities & Maths and science. \\
\hline Technology & Availability & $\begin{array}{l}\text { Applications for research purposes such as Google forms } \\
\text { to collect data, spreadsheets to analyse data, and photo } \\
\text { processing. }\end{array}$ \\
\hline $\begin{array}{l}\text { School \& } \\
\text { classrooms }\end{array}$ & $\begin{array}{l}\text { Classroom } \\
\text { environment }\end{array}$ & $\begin{array}{l}\text { School policies and classroom design that allow students } \\
\text { to freely move in school to collect data and communicate } \\
\text { with others. }\end{array}$ \\
\hline
\end{tabular}




\section{How Do the Ways of Use Contribute to the Educational Purposes of Classroom}

\section{Learning Activities?}

To understand the contributions of using digital devices to educational purposes, it is essential to consider the pedagogical approach and context in which devices have been used. Therefore, I first addressed the contribution of the ways of use to the desired educational purposes as planned by the social actors, in this case the teachers, as this study did not measure students' learning outcomes. Then I classified their objectives according to Biesta (2009) theoretical framework about the three functions of education, which are qualification, socialisation, and subjectification. In the first case, the teacher adopted the five key competencies of the New Zealand National curriculum as a framework for students' learning. The five key competencies were thinking, using language, symbols, and texts, managing self, relating to others, and participating and contributing. The five competencies aligned with the three functions of education. In case two, the objectives were extracted from the data; they were increasing students' knowledge and understanding about science, undertaking and understanding multi-phase projects, and working collaboratively. These objectives aligned with the qualification and socialisation functions of education. Figure 6.3, below, shows how the uses contributed in each case. Table 6.11, below, synthesises the contributions of the two cases; the synthesis will be used in the model developed in this study.

\section{Figure 6.3}

The Contribution of Uses to Educational Purposes in the Two Cases

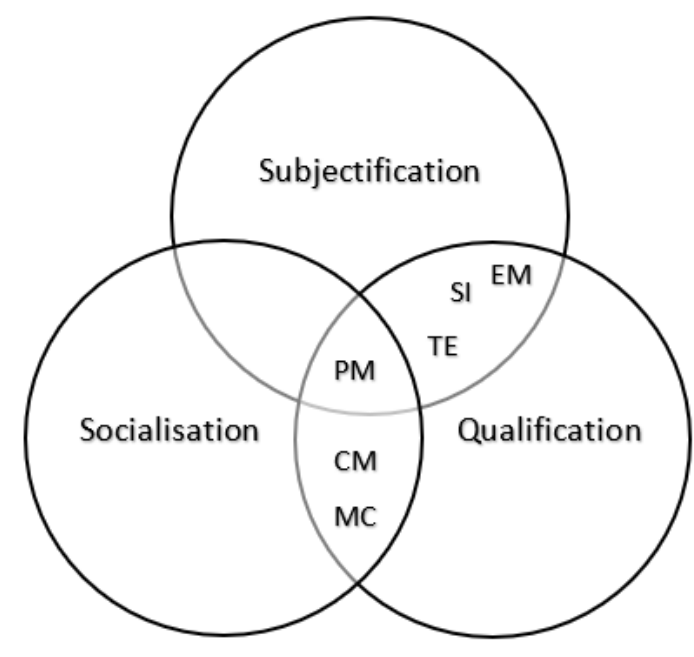

Case one

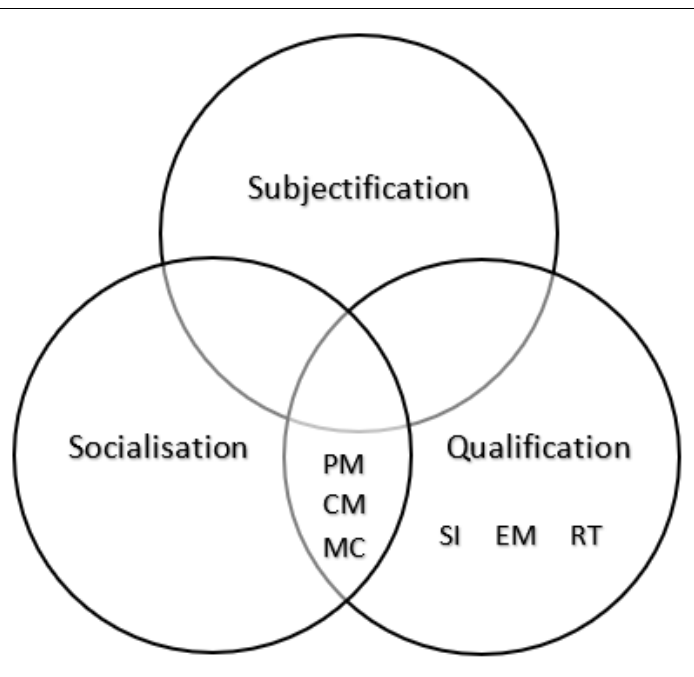

Case two

Note: SI: Source of information. MC: Means of communication. PM: Production medium. RT: Research tool. EM: External personal memory. CM: Collective memory coordinator. TE: Trial-and-error learning space. 
Table 6.11

Synthesising the Contribution of the Ways of Use to Educational Purposes

\begin{tabular}{|c|c|}
\hline Use & Use contribution to educational functions \\
\hline $\begin{array}{l}\text { Source of } \\
\text { information: }\end{array}$ & $\begin{array}{l}\text { Qualification } \\
\text { - Acquisition of knowledge. } \\
\text { - Understand and undertake multi-phase projects. } \\
\text { Subjectification } \\
\text { - Seek and use knowledge for problem-solving. } \\
\text { - Develop an understanding, make decisions (thinking). }\end{array}$ \\
\hline $\begin{array}{l}\text { Means of } \\
\text { communication }\end{array}$ & $\begin{array}{l}\text { Qualification } \\
\text { - Seek and use knowledge for problem-solving. } \\
\text { - Communicate information, expertise, and ideas } \\
\text { Socialisation } \\
\text { - Work collaboratively. } \\
\text { - Share ideas and recognise different points of view. } \\
\text { - Make connections and contribute as a group member. }\end{array}$ \\
\hline Production medium & $\begin{array}{l}\text { Qualification } \\
\text { - Make decisions. } \\
\text { - Use and create knowledge for problem-solving. } \\
\text { - Present information, expertise, and ideas. } \\
\text { - Understand and undertake multi-phase projects. } \\
\text { Subjectification } \\
\text { - Manage projects and deal with challenges. } \\
\text { Socialisation } \\
\text { - Work collaboratively to achieve common goals. } \\
\text { - Contribute as a group member. } \\
\text { - Share ideas and recognise different points of view. }\end{array}$ \\
\hline $\begin{array}{l}\text { External personal } \\
\text { memory }\end{array}$ & $\begin{array}{l}\text { Qualification } \\
\text { • Acquisition of knowledge. } \\
\text { Subjectification } \\
\qquad \quad \text { Thinking and making decisions. }\end{array}$ \\
\hline $\begin{array}{l}\text { Collective memory } \\
\text { coordinator }\end{array}$ & $\begin{array}{l}\text { Qualification } \\
\text { - Seek and use knowledge for problem-solving. } \\
\text { - Present information, experiences, and ideas. } \\
\text { - Understand and undertake multi-phase projects. } \\
\text { Socialisation } \\
\text { - Work collaboratively to achieve common goals. } \\
\text { - Share ideas and recognise different points of view. } \\
\text { - Make connections and contribute as a group member. }\end{array}$ \\
\hline $\begin{array}{l}\text { Trial-and-error } \\
\text { learning space }\end{array}$ & $\begin{array}{l}\text { Qualification } \\
\qquad \quad \text { Think and make decisions. } \\
\text { Subjectification } \\
\qquad \quad \text { Manage projects and deal with challenges. }\end{array}$ \\
\hline Research tool & $\begin{array}{l}\text { Qualification } \\
\text { - Acquisition of scientific knowledge. } \\
\text { - Understand and undertake multi-phase projects. }\end{array}$ \\
\hline
\end{tabular}




\section{How Do Students Use Digital Devices for Educational Purposes in Primary Classrooms?}

To answer the overall research question, the findings of the three sub-questions were synthesised into the Using Devices for Classroom Learning Model (UDCL) (Figure 6.4, below). This model provides an understanding of the factors that shape students' uses of devices and the contribution of the uses to the educational purposes of the social actors in classroom settings.

The starting point of the model is the apparent uses that can be distinguished while students undertake their learning activities in classroom settings. By synthesising the uses in the two cases and the connections between them, seven ways of use are identified: source of information, means of communication, production medium, external personal memory, collective memory coordinator, trial-and-error learning space, and research tool. There are two main characteristics of these uses: first, they vary from one context to another, which means they do not necessarily occur in all classrooms in the same way or the same number. Second, there are connections between the uses, which means they trigger or can be triggered by each other.

The model classifies five groups of factors that enable and shape the uses. The groups of factors are related to (1) educational system; (2) school and classroom; (3) digital technologies; (4) teacher; and (5) student. The factors are interrelated and have a dynamic nature, which means that they interact together and mutually influence each other while interacting. Based on the dynamic emerging nature of the complex systems, this model keeps the door open for other factors that might exist in other contexts or systems.

The interacting factors in the classrooms shape different ways of using digital devices to achieve desirable educational goals or objectives. The model, based on Biesta (2009) framework, classifies the educational goals of classroom learning activities to serve three educational functions: qualification, subjectification, and socialisation. Using digital devices can contribute to these domains but the contributions are uncertain and context-dependent. These contributions are part of collective efforts, which all the factors synergically participate in. In other words, the ways of use and their possible contributions reflect the interactions of different factors pursuing desired educational benefits.

In the next chapter, I discuss the findings of this study in light of the literature and theoretical framework. 


\section{Figure 6.4}

Using Devices for Classroom Learning Model (UDCL)

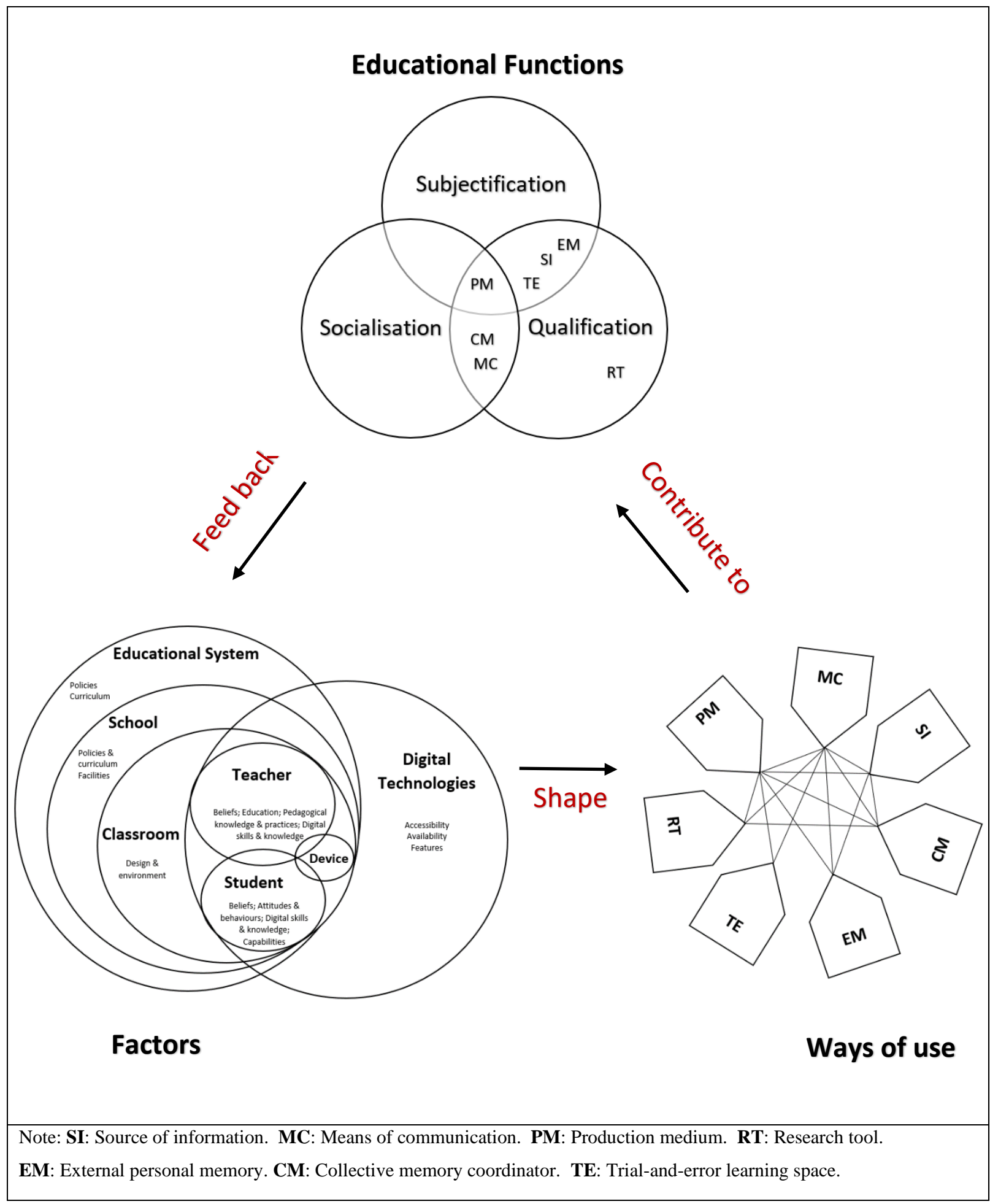




\section{Chapter 7 Discussion and Conclusion}

The purpose of this study was to describe and understand how upper-primary students (Years 7 and 8) use their digital devices during classroom learning activities. Complexity Theory (Boulton et al., 2015) was adopted to develop a holistic understanding of the phenomenon. A qualitative case study approach was used to collect data from two classrooms from two schools in New Zealand. Two teachers and seven students participated in the study and were observed while conducting four learning activities through two school terms. Data collection included classroom observation, semi-structured interviews, informal interviews, group interviews, think-aloud interviews, screen video records, and artefacts. The overall research question was: How do students use digital devices for educational purposes in primary classrooms? To answer this question, three sub-questions were addressed:

1. In what ways do primary students use their digital devices in classroom learning activities?

2. What factors shape the ways of use in classrooms?

3. How do the ways of use contribute to the educational purposes of classroom learning activities?

This chapter discusses the findings in light of the existing research studies and the theoretical framework. Then it discusses the implications and limitations of the study, followed by recommendations for future research and conclusion.

\section{Discussion of the Findings}

This section discusses the findings using Complexity Theory lenses and literature. The section is organised based on the seven characteristics of the complex system, explained in the literature review chapter. These seven characteristics are classified in relation to research questions which focused on the ways of use, the factors, and the contributions.

\section{Using Devices in Classrooms: Emergence and Self-Organisation}

"Patterns emerge from self-organisation among interacting agents" and "emergence applies to both processes and outcomes" (Patton, 2015, p. 147). This study identified seven patterns of use that emerged from self-organisation processes among different elements, or agents, in the classroom. These emergent uses were, at the same time, the processes that contribute to educational purposes, as will be discussed later in this chapter. It could be argued that selforganisation and emergence happen with "no central control or leader" (Mitchell, 2009, p. 
12) and teachers or school leadership impose control on students' behaviours. But, as argued in the literature review chapter, leadership in social systems, and what represent it, is part of the system and not an external force (Boulton et al., 2015). What also makes these uses an emergence of self-organisation is that they are not guaranteed to exist in all classrooms, or complex systems, as will be discussed shortly. Emergence differs from system to system because factors and their interactions differ from context to context. Hence, using devices differs from classroom to classroom. Furthermore, what emerges from self-organisation is not beneficial by nature; other features of the system, such as the order of the event, are what make it good or not (Boulton et al., 2015). What this study emphasises is that the ways of use are not beneficial or effective by nature; the question about using devices should shift from 'is it effective?' to 'how could it be effective?' (Biesta, 2007).

Among the seven uses identified in this study, four were explicitly, and sometimes widely, addressed in the educational research, which included using the devices as a source of information, means of communication, production medium, and research tool. The other three uses, which include as an external personal memory, collective accessible memory, and trial-and-error learning space, were implicitly addressed or overlooked in the educational research.

\section{Ways of Use Explicitly Reported in Educational Research Source of information}

The findings identified using devices as a source of information for classroom learning. The research reported that online digital resources have become a main source of information for school students (Malliari et al., 2017; Mason et al., 2018) and in some contexts students' preference to use them instead of printed resources increases as the year level increases (Lanning \& Turner, 2010). There is a rich body of literature that addresses the interactive use for seeking and using online information. Most of these studies were conducted in the field of library and information science. Abbas (2017) classifies different research areas that explore children's information behaviours and information literacy and how utilising information technologies influences children's social and cognitive domains. These areas include research about everyday life, information seeking, health information seeking, and information retrieval.

Some studies and a model developed in the field of information and library studies support the findings of my study. The 'Radical Change Typology' developed by Dresang and 
Koh (2009) classifies the characteristics of how youth seek information; it includes "obtaining information through a variety of media sources, demonstrating a preference for graphic and visual information, multitasking, seeking information nonlinearly and nonsequentially, developing self-defined and controlled paths" (p. 29). That aligns with the description of how the participants in my study used different forms of resources, such as text, video, and images. In addition, the characteristics mentioned in the model "sharing, and creating information collaboratively, forming new types of social networks, participating in community engagement" (Dresang \& Koh, 2009, p. 29) were consistent with the connections addressed in my study with other uses such as a means of communication, or collective memory. However, despite the use being widely reported in classroom research (Click \& Petit, 2010; Falloon, 2017b; Geer et al., 2017; Zilka, 2020), it was less conceptualised (Varier et al., 2017).

Understanding and conceptualising students' use of devices as a source of information is useful because it clarifies the nature of this use in classrooms settings compared to other contexts. For example, a literature review in library studies, that was conducted in 2005 , showed that school-age children were impatient and preferred browsing instead keyword searching; faced difficulty in choosing keywords and evaluating the quality and the relevance of the information; and preferred to use familiar websites and look for one correct answer (Kuiper et al., 2005). That contradicts the findings of my study, which showed that the participants spent several class sessions searching different websites and using keywords and questions. They were selective when choosing the sources to be used in their scientific research. The developments of digital technologies since 2005 could explains the contradiction, however, the context of the inquiry is important as well. The current study was conducted in a supervised context, that is, a classroom, and using devices as a source of information was part of structured learning process. Additionally, students were learning search skills that enabled them to avoid the reported behaviours. To conclude, understanding the use of devices as a source of information in classroom settings could shed light on the role of supervised searching which assists in planning and designing classroom learning activities.

\section{Means of communication}

In my study, two types of communications related to using digital devices were distinguished: communication through the devices and the communication about the devices. There is a rich body of literature that focuses on communication through the devices in different contexts 
such as e-learning, distance learning, and social networking at informal settings with a focus on adult learners, which are all out of the scope of my research. This discussion addresses research that is related to formal classroom settings and communication for academic purposes. However, most of the research that focused on communications within classroom settings were conducted in higher education settings, which indicates a gap in the literature. For example, Froment et al. (2017) reviewed 96 studies conducted between 2006 and 2016 about using social networking for communication between students and teachers. They found that only $4 \%$ of studies were conducted in elementary education settings. My study shed light on this use in primary classroom settings.

My study found that participants used and valued communicating through devices for feedback. For example, Kathy's class communicated through the devices mainly for feedback. The research reported using the computers in classrooms for feedback (Adesina et al., 2014; Alcoholado et al., 2016; Dasgupta et al., 2019; Wilson \& Czik, 2016). In my study, participants exchanged feedback on their blogs and assignments with their peers and teachers, which is a common practice in other primary schools in New Zealand (Falloon, 2015). Similar feedback practices were reported by Alhinty (2014). Young English learners aged 910 years old in the UK used their iPads for blogging and communicated with their teachers and peers virtually to exchange feedback and greetings. In Tess's classroom, the participants communicated through the devices with their peers to organise their group work during the scientific research learning activity; they used emails and social network applications. They also communicated with the community inside and outside the school to inquire about activities related to their leadership projects by using email. Baser et al. (2017) describe how seventh-grade students in Turkey communicate through devices to organise their projectbased learning activities and group work. They also receive instant feedback from their teacher, which was considered one of the benefits of using digital devices in Kathy's and Tess's classes as well. By analysing the findings of the two studies using the UDCL model, there were connections between using the devices for communication and using the devices for production and as a personal memory. Freeman et al. (2016) consider that publishing and sharing student products using blogs is a way of communicating ideas with others. This view aligns with Kathy's point of view that sharing and publishing digital products online is to communicate students' ideas, which shows a connection between using the devices for communication and production. A contribution of my study is it explicitly provides examples of how using devices as a means of communication was connected to all uses. 
With regard to communication about using digital devices, this could be an overlooked type of communication in literature. My study showed that using devices created a special type of communication that would not happen if devices did not exist. In the collaborative learning environments in my study, there were active communications related to exchanging digital skills and knowledge. Lofthus and Silseth (2019) state that digital devices, tablets in their case, "provide different ways of interacting, both in relation to the tool and to group dynamics" (p. 287). In their study, they did not explore communications about using devices as my study did; rather they studied the communication that happened about digital resources between students in a collaborative environment. My study, by conceptualising this use, could serve as a base for future investigations.

\section{Production medium}

My study reported that students used their devices to create digital and non-digital products. The research reported similar products to what was observed, which include digital stories (Girmen et al., 2019; Kimbell-Lopez et al., 2016), information projects (Koh, 2013; Koh et al., 2019), graphic design (Hsu et al., 2016), 3D modelling (Yildirim, 2018), music (Kim, 2013), and blogging (Alhinty, 2014; Freeman et al., 2016). There were other products reported that were not observed in my study, such as coding and creating digital games (Akcaoglu \& Green, 2018; Ejsing-Duun \& Skovbjerg, 2016; Liao et al., 2016; Melander Bowden, 2019), non-digital products using 3D printers (Hansen et al., 2019), animated digital stories (Liao et al., 2016; Yildiz Durak, 2018), and digital media (videos) (Spante, 2019). The systematised review conducted in my research shows that most of the students' products studied were part of an intervention. Few studies show how students produce artefacts using devices without research interventions (Cerratto Pargman et al., 2018; Dahlström, 2019; Geer et al., 2017). My study contributes to our understanding of using devices as a production medium.

Abbas (2017) pinpoints that what is also limited is the research exploring the complexity of the production process of digital artefacts. A significant model developed by Koh (2013) describes the information-creation process. Her model emphasises the iterative and nonlinear nature of the process and focuses on the relationship between using the devices as a source of information and as a production medium. Recently Koh et al. (2019) extended the model to explicitly address the relationship between using the devices as a source of information, a production medium, and trial-and-error learning. Their model, which is called the 'Spiral making process mode', shows that the non-linear nature of the production process 
supports the findings of My study. However, the model developed in this study, the UDCL model, extends the knowledge about the complexity of using devices in classroom settings by identifying production medium use alongside six uses, the connections between them, the factors shaping them, and their contribution to educational purposes.

\section{Research tool}

The participants in Tess's classroom used their devices to collect data. Zacharia et al. (2016) designed an experiment to compare using mobile devices (tablets and smartphones) with sketching and note-taking to collect data about plants. The intervention was designed using the inquiry-based approach and was conducted with fourth-grade students from Cyprus. That study found that using the devices as a tool for data collection improved students' conceptual understanding of plants compared to traditional ways. That study did not report other ways of use during the experiment. For example, the main source of information was the teacher, the students did not communicate through the devices, and their artefacts were not produced using the devices.

A study conducted by Falloon (2017b) described using iPads for experimentation and analysing data. The teachers designed science workshops about energy for Year 5 and 6 students in a school in New Zealand. The students were expected to carry out self-directed inquiries by undertaking whole experiment procedures including planning, executing, recording, analysing, and evaluating outcomes. The students used different apps to search for information, save and retrieve their progress, and communicate through the devices to report and share their findings. Falloon's study shows an example of possible connections between using the devices as a research tool and using the devices as a source of information, means of communication, and collective memory. The findings observed in my study mirror those of previous studies that have examined using devices as a research tool. What the current study adds is manifesting the factors shaping it and its contribution to students' qualifications.

\section{Implicit Ways of Use}

\section{Personal and collective memories}

My study identified two ways of use related to using the devices as memories: external personal memory and collective memory coordinator. Interestingly, addressing these uses has flourished in psychology and health research but not yet in education. Wegner (1987) defines individual memory as a process in which "Information is entered into memory at the encoding stage, it resides in memory during a storage stage, and it is brought back during the 
retrieval stage" (p. 186). Wegner adds that humans tend to create "External memories" to "record items in external storage media" (p. 187). In the same study, Wegner introduces the concept of the 'Transactive Memory System', which:

In a group involves the operation of the memory systems of the individuals and the processes of communication that occur within the group. Transactive memory is therefore not traceable to any of the individuals alone ... Rather, it is a property of a group ... In short, transactive memory derives from individuals to form a group information-processing system that eventually may return to have a profound influence upon its individual participants" (p. 191).

These pre-digital age definitions were revived in the digital age to study the relationships between using information and communication technologies and humans' memory in psychology. Sparrow et al. (2011) wrote, "The Internet has become a primary form of external or transactive memory, where information is stored collectively outside ourselves" (p. 776). There was more focus on the transactive memory that the Internet represents than the external individual memories that technologies can provide (e.g., Firth et al., 2019; Storm \& Stone, 2015).

In education, Davis et al. (2013) define memories as "discernible patterns of activity among the agents that come together in more complex collectives" (p. 78). They distinguish between 'personal memory', which is part of the human neurological system, and 'collective memory', which is more related to the social system. In my review, I could not find links between these concepts and using technologies in educational research.

This study found patterns of use that can be described as an external personal memory where the children save their information in external personal storage media (e.g. accounts) and retrieve it when needed. In addition, the findings of this study support the studies that consider the Internet as transactive memory. The students in this study used their devices as a coordinator to create collective accessible memory that involves collaborative creation and shared access for saving and retrieving. Some studies describe how primary students use Google Docs to create and access collaborative projects to be used later for presentation (e.g, Falloon, 2015). But the use was described but not conceptualised. Other studies address cloud computing and define it as "the physical structure of a communications network, through which data is saved and stored in large data centers and can be accessed anywhere, at any time, and from different devices" (Hartmann et al., 2017, p. 190). But most of these studies 
focus on higher education contexts and address cloud computing as a static structure. My study identified it as a dynamic way of using digital devices and emphasised the collaborative side of creating it.

\section{Trial-and-error learning space}

Using digital devices as a trial-and-error learning space was a significant way of use. In Kathy's class, this use was not a blind trial-and-error experience, because it was deliberated and directed (Biesta, 2007). The students were conscious that they were challenging themselves, making mistakes, and taking risks. They reflected on this use during the evaluation phases after the workshops, which manifested students' awareness of the importance of this use as part of managing-self during the learning experience. Literature indicates that making mistakes and trial-and-error practices have benefits for learning (Cyr \& Anderson, 2015). However, this form of learning is rarely linked to using digital devices in educational research.

In this study, trial-and-error learning on the devices was connected to production, particularly when students were learning digital skills. This explains why I did not observe this type of use in Tess's classroom as their learning activities did not involve learning new digital skills. That does not mean that this use did not happen previously. While this use was not addressed explicitly, research reports that children start their problem-solving attempts with trial-and-error practices when using robotics kits for the STEM approach (Sullivan \& Heffernan, 2016). For writing, students prefer writing on their devices because they do not feel afraid of making mistakes while trying creating their stories (Dahlström, 2019). In the context of higher education, most first-year college students use the trial-and-error method to create computer games (Frydenberg, 2015). Recently Koh et al. (2019) explicitly added the trial phase to their model about digital production behaviour.

One of the ways of use that was reported in the literature and was not reported in my study was gaming (Geer et al., 2017; Nogry \& Varly, 2017; Starkey et al., 2018). This use was not reported in my study because none of the learning activities observed included educational games. The systematised review of this study showed many experimental studies that addressed that use. Adding this use, as derived from literature, enriches the UDCL.

A possible reason for not explicitly addressing the three ways of use in educational research can be drawn from Blaikie (2009) view that humans usually do not reflect or give attention to most of their everyday activities. He adds that only when these activities are 
disrupted do people start giving them attention. Saving, retrieving, and trying application features become taken-for-granted practices in the everyday use of technology. In research, these uses are also taken for granted because they are usually hidden or implicit when focusing on intervention in experimental conditions. Blaikie (2009) argues that it is the role of the social scientist to explicitly address these overlooked activities and identify them.

\section{Using Devices in Modern Classrooms}

This study showed the different roles that one device can take in modern classrooms. Identifying and conceptualising these uses help in reflecting on how our students use them for learning and how we integrate them in classroom pedagogy. For example, what ways are needed for the learning activity? What factors do I need, as a teacher, to enable these uses? How would these uses contribute to achieving the learning goals of the learning activities?

The connections between uses should be taken as possible connections. These connections result from how the teacher designed the activity. There are some examples in literature that show that some uses did not lead necessarily to other uses (Kang, 2019; Zacharia et al., 2016).

\section{Factors: Considering the Complexity}

This study identified factors related to five elements that shape or enable the uses of digital devices. The five elements were the educational system, the school and classrooms, the teacher, the students, and the digital technologies. Attributing the factors to the elements is contextual, which means that in other contexts some factors could be attributed to other elements. For example, choosing learning activities is related to the teachers in the New Zealand educational context, while in other contexts learning activities are determined by the national curriculum of the educational system.

The discussion of this section is based on Complexity Theory' assumption, that " $\mathrm{A}$ complex system does not comprise simple, discrete parts. Rather, it is itself a collective of dynamic and similarly complex systems" (Davis \& Sumara, 2001, p. 88). Therefore, an element in my study should not be understood as a chemical element, that is, a substance that cannot be broken down into simpler substances. Rather, the element is a unit that acts as an agent (Boulton et al., 2015) where different factors are related to it. Figure 7.1 below shows the five elements which could be considered as a system or nested systems in other studies. It shows the focus of this study, highlighted with red, which is the interactions between the students and their devices, particularly when the students use the devices for learning 
activities in classrooms. These interactions are embedded within nested overlapping systems. The student and the devices are elements in the classroom system, the classroom system is part of the school system, and the school is part of the educational system. At the same time the devices belong to the digital technologies system where many interactions happen that are out of the scope of this study.

\section{Figure 7.1}

The Interconnected Elements and the Focus of the Current Study

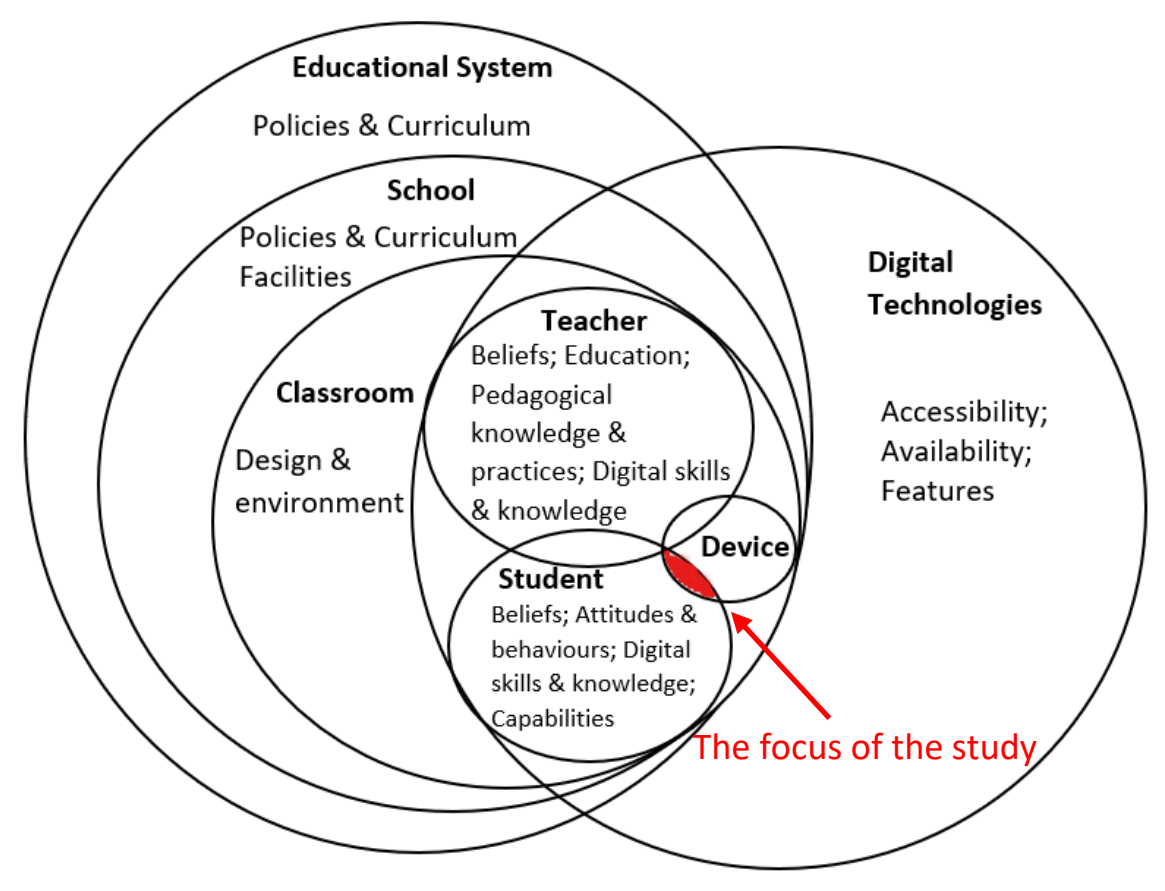

The overlapping circles in Figure 7.1 are used to emphasise the nature of the elements and factors interacting in complex systems. There are many variables that are constantly changing and changing others (Boulton et al., 2015). The relationships between them are dynamic and self-organising and emerging (Kuhn, 2007). The next sections discuss further the features of the factors in light of the characteristics of complexity systems, which provides a deeper understanding of using devices in classrooms.

\section{Systemic and Synergistic Relationships}

This study argues that complex systems are systemic (Boulton et al., 2015). That implies that we cannot understand the uses and their contributions without understanding the interconnectedness of the factors. That requires first identifying the factors then describing how they shape the uses collectively and influence each other. Therefore, identifying the 
factors for each use was not to disconnect them but to see the connections between them. Therefore, this study did not consider the factors as constant variables interacting in a linear cause-effect way (Patton, 2015). This study considered them as dynamic variables interacting in a nonlinear way, and, at the same time, acknowledged that there were possible factors that data collection methods did not capture.

Synergistic relationships mean that changes happen as a result of synergistic causes, not merely a single cause (Boulton et al., 2015). That is, each use results from many factors interacting together. For example, available digital technologies provided private accounts secured by passwords to save and retrieve information; the students had skills to access these accounts and use them properly; the teachers provided instructions and lessons to their students of how to manage their accounts and create activities that require saving and retrieving practices. All these factors worked synergistically to shape using devices as a personal memory. This concept challenges the deterministic causal mechanical mindset that aims to provide evidence of the effectiveness of interventions utilising digital devices through experimental methods. The problem with this mindset is that the effectiveness to make it happen requires strict conditions in closed systems, which do not apply to the open systems of educational contexts (Biesta, 2015) such as classrooms and schools. In this study, teachers' pedagogy, instructions, and guidelines, even if they were evidence-based, could not benefit the learners if they did not adopt them. Bob showed commitment while using her teachers' guidelines and helped her to do her tasks, while Mitchel did not follow these guidelines because he preferred other ways that suited his capabilities. To conclude, in complex systems, the synergy of the factors, not a single factor, and the relationships between them, are the key factors that shape the outcomes.

Another benefit of the synergy concept is that it explains the mutual influence of the elements on each other and how they interact with respect to each other. It represents the hyphen between actor-network in ANT (Latour, 1999), which means that what happens to any element will affect the whole system. An example from Tess's class was that pedagogy influenced students' digital skills, such as teaching Year 7 students how to communicate through devices; and students' digital skills influence pedagogy, such as students from Year 8 who undertook their leadership projects independently while Year 7 students were supervised. 
The other argument is that "We cannot and should not assign a percentage 'weighting' to the influence or effect of each of these factors because they interact together in complex ways" (Boulton et al., 2015, p. 37). A reason for not weighting the factors is that one factor, that might be marginalised could hinder or interrupt the efforts of other factors. For example, the findings of my study might lead us to conclude that factors related to teachers are the most significant factors in shaping uses. But as some incidents showed, teachers' efforts to enable students to use devices were interrupted when a password function did not work and the students could not log in to their devices. Other studies described how breakdowns, which stopped digital devices from working in classrooms, interrupted significant routine operations of schools (Alirezabeigi et al., 2020). Another reason for not weighting the influence of the factors is that it is context-dependent. My study was conducted in New Zealand where teachers have the agency to design the classroom curriculum and choose learning activities. In other contexts, the factors related to the educational system could have more influence on shaping uses. For example, when a unified central curriculum imposes the types of learning activities and restricts use of devices in classrooms, it will not be accurate to claim or generalise that the factors related to teachers are the most important factors in shaping use of devices in classroom learning activities.

However, 'not' weighting the factors does not mean 'not' acknowledging the predominant factors; in this study the factors related to teachers were dominant. Many studies reported factors related to teachers that influence using devices in classrooms (Cerratto Pargman et al., 2018; Dahlström, 2019; Kim \& Jang, 2015; Varier et al., 2017; Zilka, 2020). That aligns with the findings of my study, which showed how teachers' beliefs, pedagogical knowledge and practices influenced using devices. That might explain the research interest in developing models related to teachers' integration or utilisation of devices in classrooms. A prominent model is the Technological Pedagogical Content Knowledge (TPACK) (Koehler et al., 2013) framework, which was originally designed to explain how teachers' knowledge domains influence teaching with technology.

The TPACK framework emphasises the complex nature of teaching and addresses three domains related to teachers' knowledge: content, pedagogy, and technology. The framework then addresses the interactions between these three domains of knowledge. In addition, teachers' pedagogical content knowledge results from the interactions between their beliefs and their knowledge (Hashweh, 2006). It was interesting in my study to see the mixture of beliefs that shaped Tess's beliefs about learning, which in turn shaped her 
pedagogical practices. Tess did not adopt a particular theory or approach for her teaching. When she was asked about what is learning, she reported "making meaning and connections", which is close to the cognitive and constructivist point of view about learning; and 'developing skills' that make learners ready for real life, which is close to Dewey's pragmatic educational philosophy (Dewey, 1998); and 'gaining information', which is a more traditional point of view about learning. The collective belief interacted with her growing knowledge and experience gained from workshops and teaching and was part of the factors that shaped the ways of use.

One of the critiques of TPACK framework was that it focused on teachers' knowledge rather than students' learning or students' use of digital technologies (Cherner \& Smith, 2016). Another model framework, the SAMR model (Puentedura, 2009), was explained in the literature review chapter and was employed by one of the participants, Kathy, in her teaching. It also focuses on the utilisation of devices from the teachers' side, not the learners'.

\section{Multi-Scalar Factors}

In complex systems, factors are classified into large-scale factors and local direct factors (Boulton et al., 2015). To understand how the ways of use were shaped, it was important to include both the large-scale factors alongside the direct local factors. In this study, both largescale and local direct factors were found to shape use of devices by students.

The literature review chapter discussed some large-scale initiatives or projects in different educational systems related to using digital devices in classrooms (Ale et al., 2017; Heinrich et al., 2020; Kennedy et al., 2016; Starkey \& Zhong, 2019). The outcomes of these initiatives varied. One of the reasons might be related to large-scale factors such as the educational system and its policies. An example from my study is related to using devices as a source of information, a research tool, and production medium. The New Zealand Curriculum, a large-scale factor, is flexible and does not impose a certain source of information, such as textbooks, on teachers or students and alternative assessments are used in primary education (Ministry of Education, 2007). To undertake experiments and scientific research, the students in Tess's classroom used devices as the main source of information. That was not the case in Zacharia et al. (2016) experimental study; while the students used the devices as a research tool, the main source of information was Cyprus's primary science textbook and products were created using pen and paper. That is similar to the context where 
I came from, Palestine, as teachers of most of the subject areas were limited to using textbooks as the main source of information; students' products had to be on paper to be evaluated. In such contexts, what hinders using devices as a source of information or as a production medium are educational systems' policies related to agency and assessment, which are both large-scale factors.

At the same time, direct local factors shaped using devices. An example was the factors related to students, which shaped the uses even if all other factors were similar. In the systematised review of this study, a few studies reported factors related to primary students compared to studies that reported other factors (Dahlström, 2019; Geer et al., 2017; TiradoMorueta et al., 2020; Varier et al., 2017; Zilka, 2020) (see Table 2.4). A literature review reported that students' prior knowledge, attitudes, age, and gender influence their information-seeking behaviours (Kuiper et al., 2005). My study extended our knowledge about factors related to students that shape seven ways of use. Factors included students beliefs about digital devices, attitudes and behaviours, digital skills and knowledge, and capabilities. Factors related to students' age and gender could not be addressed due to the limited sample size of the qualitative research. To conclude, how students use devices in classrooms is not only shaped by local factors such as teachers or students, but is also shaped by large-scale factors such as educational systems and their policies.

\section{Diversity, Variation, and Fluctuations}

In complex systems "diversity, variation, and fluctuations lead to resilience and adaptability" (Boulton et al., 2015, p. 39). There could be micro-diversity which means "variation within a type" and macro-diversity which is "variation between types" (Boulton et al., 2015, p. 242). To report fluctuations, longitudinal large-scale studies are needed. Identifying interacting factors in a complex system in advance could be problematic (Patton, 2015). The reason is that they could be too many, diverse, and overlapping, and some of them will not be acknowledged unless they become absent (Alirezabeigi et al., 2020; Boulton et al., 2015). But this diversity and variation are what lead to resilience and adaptability. In my study, diverse factors related to different elements influenced using devices in classroom learning and made these uses exist and persist.

Factors related to digital technology are a good example of the variation. Most of the studies in the systematised review in Chapter 2 designed and examined digital products, such as applications and games, for classroom learning; and some used available applications on 
the Internet. That is an example of the ongoing growth and variety of digital innovations. Some studies reported factors, related to digital technologies, to influence using devices such as accessibility (Dahlström, 2019; Varier et al., 2017) and the features and quality of devices (Geer et al., 2017; Varier et al., 2017; Zilka, 2020). The accessibility was reported in my study while the quality of the device was not, as the participants in both schools used Chromebooks, which meant it was not possible to compare. However, other factors were identified in my study in addition to linking these factors to certain ways of use, which makes a noteworthy contribution to understanding using devices by students. That includes features such as 'undo' to trial-and-error learning and spell check to the production medium.

As Tess reported, limited features provided by digital technologies in the past did not fit with classroom learning and did not encourage her to continue using them. These days, diverse features and applications make it possible to use devices and technologies for different purposes. In pre-digital classrooms, students used other materials, such as books, as a source of information. They communicated through letters with their teachers and peers. They used trial-and-error on blackboards and created crafts using pens, papers, and scissors. They used their diaries as external personal memories and if they wanted to create shared memories, they might use a camera to take a photo and share it with each other. Now, one device is doing all these roles. Hence, one of the reasons that made these patterns of use exist and persist in classrooms and made teachers and students adapt to it is the variety of features that fit different purposes.

\section{Context, Events, and History}

The findings showed that there were different factors related to the context: the educational system, school, and classrooms where other elements were part of it, which are teacher and students. The factors related to digital technologies may be cross-contexts because of the nature of the Web 2.0 technologies. Studies investigated initiatives to integrate digital devices in classrooms reported that the cultural, socio-economic, and contextual domains were influential factors in the integration (Ale et al., 2017; Heinrich et al., 2020; Kennedy et al., 2016). While some cultural and contextual factors were identified in my study, the socioeconomic factors were not detected because of the limitations of the methodology, which will be discussed later.

The context of the educational system shapes how students used the devices. For example, primary students in Madagascar used their devices at school mainly as a textbook 
and rarely used them for production or other uses (Nogry \& Varly, 2017); when students from Cyprus used the devices as a research tool they did not combine this use with other uses (Zacharia et al., 2016). Studies conducted in New Zealand reported similar uses found in the current study, such as using for communication, production, seeking information, and as a research tool (Falloon, 2015, 2017a, 2017b; Starkey et al., 2018). This explains why imposing exported digital innovations does not necessarily generate the expected uses or outcomes (Claro et al., 2013; Kang, 2019).

The order of the events and the history of the system also shape features of the complex system. Understanding the order can help in implementing pedagogical approaches to integrate digital devices. Kathy and Tess reported that their digital skills and pedagogical knowledge changed due to the professional development courses on how to use digital devices in teaching and their initial teacher education. These changes altered their knowledge and beliefs about the potentials of digital technologies. The students reported that changes in teachers' digital skills enhanced their learning with digital devices. Research showed that preparing teachers for using devices is a prerequisite to the successful integration of digital technologies in schools (Geer et al., 2017; Severin \& Capota, 2011). Hence, not only are the presence or the interactions between the elements important, but also the order in which events happen is important. For example, students should not be expected to create digital products if their digital skills are not developed enough. Tess noted, with regard to using devices as a source of information, that it was better to start with limited options with supervised teaching to enhance students' capabilities before moving to more open, free use. This is supported by research findings that efficient information seeking is not born behaviour but should be learnt (Limberg \& Alexandersson, 2017).

This study found that a classroom environment that enabled students to communicate and collaborate was one of the factors that shaped using the devices as a research tool, production medium, and means for communication. That matches findings from other studies that giving students agency and allowing reasonable noise and communication are crucial to enable collaborative production of digital artefacts (Dahlström, 2019; Walton et al., 2019) and encourage students to use devices in classrooms in general (Zilka, 2020).

\section{Self-Regulation Process}

Self-regulation is the processes, usually everyday activities, that maintain the features of the system (Boulton et al., 2015). This study explored using devices in natural settings without 
interventions; all the learning activities observed were regularly conducted in the classrooms. The teachers in both classrooms had experience in implementing the learning activities which created patterns of interactions that preserved the dynamics of the classrooms.

One of the factors that contributed to the self-regulation processes was school policies that organised how the school community should use devices in school. Kathy attributed students' commitment while using their devices in classrooms and the lack of off-task behaviours to students' commitment to the 'Kawa of care' agreement. Signing this agreement and checking the students were aware of it helped in organising the uses of the devices and maintaining the dynamics of use in the classroom. Research suggests that "clear communication of policies and protocols with the school community" (Janssen \& Phillipson, 2015 , p. 1) are essential to using devices in schools. However, researchers have not treated the influence of these policies on the stability of using devices in primary schools in much detail; further exploration is needed, especially with how they affect students' commitment and behaviours when using devices.

\section{Tipping Points and the Episodic Change}

In complex systems, significant changes are episodic, which means certain events, called tipping points, lead to radical changes in the system (Boulton et al., 2015). Tipping points are usually recognised after a period of time has passed since the system changed. Therefore it is not accurate or possible to claim that tipping points had happened while studying the phenomenon 'in action' during the 20 weeks spent in classrooms. However, the teachers reported tipping points that had happened.

Kathy mentioned a tipping point that affected school and classroom systems, which had a significant impact on using the devices in her classroom. This tipping point was the decision to integrate devices that was taken by the school leadership. That decision led other interactions to happen, including teacher professional training, changes in the school curriculum and policies, providing stable Internet connections, cooperation with the families the community, and the establishment of a trust to enable students to buy their own devices. This decision was a tipping point in that system because it transformed traditional teaching and learning in classrooms. Research suggests that it is not enough to provide the necessary infrastructure and teacher training to integrate digital technologies in classrooms; it is the vision or the 'ideological position' that underpins the school policy (McFarlane, 2019). This explains that the deep root of the tipping point reported in School A was the 'social justice' 
position adopted by the school leadership, which was that none of their students should be left behind.

From a wider perspective, the development and innovations in digital technologies represent tipping points in human history. Classrooms before the digital age are not similar to classrooms in the digital age (Bates, 2018). Tess expressed that her beliefs about integrating digital technologies in classrooms had changed significantly compared to 20 years ago. She used to believe that devices were not useful for classroom learning. The development, availability, and the features that made using devices easy for the teachers and children made her believe that digital devices provide unique learning opportunities for children. What happened to Tess might have happened to other educators as well. Different studies show that the features of the technology do influence the use (Varier et al., 2017; Zilka, 2020). While, as explained, it is hard to predict if a new innovation will create a tipping point in classroom learning, it is not hard to point to some innovations, such as the Internet and mobile devices, that have changed teaching and learning in today's classrooms.

\section{Contributions to Educational Purposes: More Than One Future}

The literature review chapter showed the research tendency to examine the interventions that integrate digital devices, such as teaching methods, applications, or specific ways of use in order to provide evidence of effectiveness. This study took a different approach by exploring the uses and identifying their possible contribution to educational purposes (Biesta, 2010). This study showed that there are different factors related to different elements that interact together to shape different ways of use in order to achieve desirable educational purposes. This means that using the devices became part of the dynamics happening in the classroom. Attributing the outcomes to specific factors is not possible in that "open recursive semiotic system" (Biesta, 2015, p. 206). What can be claimed are the possibilities and opportunities digital devices can offer to the educational process without deterministic assumptions. This logic helps us to see more than one future of using devices in classrooms, where each system creates its own future depending on the elements and the relationships inside it.

Each class in this study had desirable educational goals, which were classified into three intertwined educational purposes: qualification, socialisation, and subjectification. Although educational purposes mutually influence each other, it is useful to separate them for understanding and analysis (Biesta, 2009). In this study, the desirable objectives related to students' qualifications were dominant. That was expected, as enhancing students' 
knowledge and/or skills are usually the explicit declared educational purposes of formal education (Priestley \& Sinnema, 2014).

The findings showed that the contributions of the uses happened in different ways, making these contributions uncertain and non-deterministic. For example, digital devices enabled the students to seek and use the information they collected from different sources to do their scientific research and there was evidence that their knowledge about the topics they were searching about increased. This does not mean that the quality of this knowledge was the same. For example, the students, such as Mark and Alice, who used different resources were able not only to show their understanding of the topic but also to use their knowledge to create models that illustrated their understanding. While Mitchel, who limited his resources to videos, showed bias in using the information to support the benefits of gaming, as Tess reported. My study emphasises that the relationship between information seeking and learning is complex and shaped by students' practices of information seeking in schools (Limberg \& Alexandersson, 2017).

In this study, using the devices as a production medium, a means of communication, and collective memory contributed to students' socialisation, such as collaborative activities, sharing ideas, and making connections. That was supported by other studies that reported that devices facilitated collaborative work between students (Cerratto Pargman et al., 2018) and influenced teachers' pedagogy, leading to more collaborative activities (Geer et al., 2017). That was not the case in Kang (2019) study, which found what can be called 'Lonely Collaborative Information Behaviour'. The middle and secondary Korean students in her study divided the collaborative projects into parts and each student worked individually on their devices to complete their parts. Kang predicts that social and cultural factors related to the rigid competitive educational system in Korea has shaped students' collaborative behaviours. As in Kang's study, even if the approach was named collaborative and students created collaborative products and collective memories, that does not mean that their use contributed to their socialisation.

Consequently, it is very important to be careful when claiming benefits of using devices as there will always be unwanted side effects. The following uses are examples of the two sides of using devices. 


\section{Production Medium}

The research reported that using devices for production reinforces students' sense of empowerment (Dahlström, 2019; Koh et al., 2019; Liao et al., 2016), self-motivated engagement (Kim, 2013), and creativity (Ejsing-Duun \& Skovbjerg, 2016). Student's science knowledge and argumentation skills were enhanced when students used devices for projectbased learning (Hsu et al., 2016). Students' writing skills and computer literacy improved when students created digital stories (Girmen et al., 2019) and digital media products (Kimbell-Lopez et al., 2016). Collaborative digital media making helped students realise the potential of working in technology fields and the importance of collaborative teamwork (Liao et al., 2016). These findings support the finding of my study that using devices as a production medium contributes to the three educational purposes.

These benefits should be taken with caution. For example, Ian reported that although devices helped in speeding up the process of the production process it did not help in checking the quality of their work; they needed the teacher's influence and extra time to manually revise their work. Participants in Kathy's and Tess's classrooms were able to create documents with no spelling errors for critical reading and scientific research learning activities. The final products were error-free, but that did not reflect the observed spelling skills of the participants. Mark while typing had almost no spelling errors while Peach heavily depended on the spell check to proofread her writing. That was also the case while Bob was typing. Although using the devices contributed to creating a desirable product, that is, error-free assignments, and the spelling check function could be considered as an affordance (Dahlström, 2019), the side effect on students' spelling skills is questionable. Research shows that although students prefer using a spell check for writing (Kiliçkaya, 2018), in some cases students' spelling proficiency weakens as a result of relying on digital spell checks (Radi, 2015).

\section{Personal and Collective Memory}

The participants in this study relied on their devices to save their work and collections, making it an automated way of use. Their personal and collective memories were not limited to their accounts but extended to their blogs. Research suggests that the recording feature of the students' blogs could lead to better collaborative explanations compared to face-to-face collaboration (Stoyle \& Morris, 2017). In psychology, this use was addressed and explored. Research found that humans are adapting to the new external memories in different ways, such as remembering only what is likely not to be stored on devices, and remembering where 
information could be stored rather than the information per se (Sparrow et al., 2011). That matches the observation when Bob could not remember the information about the scientist but was able to remember where she read about him. There is evidence that saving on computers influences humans' memories and learning as humans become more dependent on them (Storm \& Stone, 2015). The students in my study considered the Internet to be the main source of information and preferred to use it over other sources. The long-term impact of this type of external memory is still debatable (Firth et al., 2019) especially with the competing pros and cons on people's lives.

\section{Source of Information}

The influence of using the devices as a source of information was explored in the fields of education and psychology. In psychology, it was found that a few days of training of using the Internet for information seeking were enough to make people dependent on it as a source of information (Wang et al., 2017) and that humans are tempted to reuse it for accessing information (Storm et al., 2017). At the same time, an association was found between Internet-based searching and poor accuracy when recalling information combined with a lack of confidence in recalling information gained through Internet searching (Dong \& Potenza, 2015). In education, research shows that there is a relationship between how students use and seek information and the quality of their learning (Limberg \& Alexandersson, 2017).

Distraction was a major challenge for both the students and teachers. This is a common concern in different educational contexts regardless of the age of students, such as in schools (Ditzler et al., 2016) and universities (Aagaard, 2015). Students in Tess's class complained that when searching for information they easily got distracted, which matches findings in psychological research. Research found links between using the Internet as a source of information and distraction; and attributed the causes to the exposure to countless information gates such as hyperlinks and notifications at the same time (Firth et al., 2019). This may be one of the reasons why distraction was not reported in Kathy's class, as the learning activities did not expose students to these information gates, and explains why Tess decided to limit topic options for novice students.

The inconsistent findings of research should not be seen as contradictions. Digital technologies are extensions to human capabilities, but these extensions always come with a price. Unlike the strict causal approach, complexity thinking looks at the conflicts in the findings as a holistic picture of what to expect when using devices in classrooms, possible 
contributions, and more than one future. The future in the complex systems is contingent and path-dependent (Boulton et al., 2015), which means that the outcomes are shaped by the collective contributions of the elements interacting in the system. Therefore, the factors that shaped the uses also shape how the contribution will look. Using digital devices alone does not guarantee the outcomes (Yelland, 2018).

The uses are seen in this study as emerging from self-organisation and maintained by self-regulation. They are outcomes of the interacting factors as well as processes that contribute to educational purposes without guaranteeing the outcomes. This position has contributions and implications discussed in the next section.

\section{Contributions of the Research}

This study has gone some way toward enhancing our understanding of using devices in classrooms. By revisiting the gap reported in the literature review chapter, it took a different theoretical approach from the dominant one in research. By adopting Complexity Theory, it studied the phenomenon from a holistic angle, and engaged ANT to report how it occurred in action. This study contributes to the studies exploring the uses in natural settings and acknowledged social accounts about learning and learning objectives. This study provides a thick description of the context, how the students used the devices, and how these uses were shaped. This study shows the socio-materiality of the devices by focusing on the possible contributions, instead of on deterministic outcomes.

This study confirms some previous findings and contributes additional evidence. It explicitly provided clear discrimination of both the apparent and overlooked ways of use and kept the door open for other uses that may emerge in other contexts. It addressed the possible connections between the uses by showing how the students consolidate them during their learning activities in classrooms. For the factors, this study identified the factors with relation to interacting multi-scalar elements in classrooms and emphasised the complex nature of the relationships between them. This study utilised the functions of an education framework (Biesta, 2009) as an approach to move from effectiveness focus towards purpose focus. The findings were synthesised into the Using Devices for Classroom Learning (UDCL) model, which is based on theoretical perspectives and empirical evidence.

The models I found in research related to using digital devices focused on information seeking and production uses (Dresang \& Koh, 2009; Koh, 2013; Koh et al., 2019). To the best of my knowledge, this is the first study that develops a model that includes the uses of digital devices, the factors that shape them, and their contributions to educational functions. 
This model can be used for future research to analyse and understand using devices in other contexts. It is flexible enough to add or remove uses depending on what emerges in classrooms. It is also flexible enough to classify the factors depending on the context and to classify the contribution depending on the objectives of social actors.

This model is a descriptive model, not a theory nor a framework. Complexity thinking assumes that "analysing the dynamics of the present will not, in general, give information as to what will emerge or how it will emerge" (Boulton et al., 2015). This means that the model is useful to analyse what is already happening or has happened, but it is limited with regard to planning or predicting the future dynamics of interventions and what would emerge from them. The other limitation is that it focuses on the uses in the formal education settings, that is, classrooms and schools. Using devices outside classrooms could create different uses with more factors and different contributions. The third limitation is that the model was designed based on empirical evidence collected in primary education settings, which means that younger or older users could produce different uses shaped by other factors leading to other contributions.

My research has several practical applications. For teachers, the detailed description of using devices for four learning activities encourages teachers to reflect on their own context and decide what can work in their context and what cannot. For school leaders and policymakers, it provides a holistic vision of the factors that influence using devices in classrooms, which could assist in anticipating realistic expectations of using devices in classrooms.

\section{Study Limitations}

The first limitation of this study is the researcher as a tool for collecting data. Although I did extensive readings and activities related to the educational system in New Zealand, I was an outsider researcher. An insider researcher with more experience in the context could address other factors and events. For example, there are factors mentioned in research that would be interesting to explore further, such as those related to home and the socio-economic status of the students. The interviews did not include questions that explored these factors. My position as an outside researcher could be an advantage and disadvantage at the same time, as, for example, I was able to distinguish and value the deep influence of the agency given to schools and teachers in New Zealand on shaping the uses compared to the central educational system in the context where I came from. Additionally, I was able to see how classroom 
designs and school policies enabled communication about devices and collecting data, which cannot happen in the traditional classrooms in my context.

The other limitation is related to the time spent in collecting data, which limited the number of classrooms that I could observe and the number of learning activities to four. Although the collected data was enough to develop an understanding of the dynamics of the uses, more time allocated to collecting would enable exploration of more ways of use, more learning activities, and/or more classrooms. The main reason for this limitation was due to prioritising depth over breadth, as I needed to deeply understand how things worked in classrooms. The other reason was being an international $\mathrm{PhD}$ student with limited time and resources.

\section{A Final Reflection}

This research was a valuable learning experience for me as a teacher and researcher. It provided an answer to my questions about using devices in classrooms and enabled me to critically reflect on teaching and learning practices in different contexts. As a teacher who came from Palestine, I always wondered why using digital devices in that context does not change learning in classrooms, and in some cases made it even worse. Why do teachers struggle to achieve the outcomes suggested by evidence-based practices? What is missing? And what do we need to consider? One of the important lessons I have learnt from my study was the deep and inevitable influence of the education system on everyday classroom learning. When students are limited to certain knowledge sources, they will not have the chance to experience learning and managing knowledge from open sources of information. When students are expected to work individually and compete with each other, they will be cautious about communicating through or about devices for learning or giving feedback to each other. When the major and preferable ways to manifest students' learning are exams and individual products, students will not be motivated to create collective memories or collaborative digital products. When classroom environment and school policies limit students' mobility they will not be able to move around to communicate and collect data to test their hypotheses. When my role as a teacher is to execute pre-designed learning activities and follow the curriculum literally, there will be no room for creativity or agency, even when using digital technologies. I learned from this study that we should not expect much from putting devices in classrooms if we do not address the three purposes of education: qualifications, subjectification, and socialisation. I learned that it is not a single factor that 
will make the desired change in education but a network of factors that will shape the emerging patterns of behaviours and outcomes in educational environments.

\section{References}

Aagaard, J. (2015). Drawn to distraction: A qualitative study of off-task use of educational technology. Computers \& Education, 87, 90-97. https://doi.org/10.1016/j.compedu.2015.03.010

Abbas, J. (2017). Children and information technology. In J. D. McDonald \& M. Levine-Clark (Eds.), Encyclopedia of library and information sciences (4th ed., pp. 839-851). CRC Press. https://doi.org/10.1081/E-ELIS4

Adesina, A., Stone, R., Batmaz, F., \& Jones, I. (2014). Touch arithmetic: A process-based computeraided assessment approach for capture of problem solving steps in the context of elementary mathematics. Computers \& Education, 78, 333-343. https://doi.org/10.1016/j.compedu.2014.06.015

Akcaoglu, M., \& Green, L. S. (2018). Teaching systems thinking through game design. Educational Technology Research and Development, 67(1), 1-19. https://doi.org/10.1007/s11423-018$\underline{9596-8}$

Alcoholado, C., Diaz, A., Tagle, A., Nussbaum, M., \& Infante, C. (2016). Comparing the use of the interpersonal computer, personal computer and pen-and-paper when solving arithmetic exercises: Comparing the use of three technologies on arithmetic exercises. British Journal of Educational Technology, 47(1), 91-105. https://doi.org/10.1111/bjet.12216

Ale, K., Loh, Y. A.-C., \& Chib, A. (2017). Contextualized-OLPC education project in rural India: Measuring learning impact and mediation of computer self-efficacy. Educational Technology Research and Development, 65(3), 769-794. https://doi.org/10.1007/s11423-017-9517-2

Alhinty, M. (2014). Young language learners' collaborative learning and social interaction as a motivational aspect of the iPad. In 2014 International Conference on Interactive Mobile Communication Technologies and Learning (IMCL2014) (pp. 64-69). IEEE. https://doi.org/10.1109/IMCTL.2014.7011106

Ali, A. (2017). The development of the learning exchange school clusters in New Zealand: An Actor Network Theory and Complexity Theory analysis (Unpublished PhD Thesis). Victoria University of Wellington, Wellington, New Zealand.

Alirezabeigi, S., Masschelein, J., \& Decuypere, M. (2020). Investigating digital doings through breakdowns: A sociomaterial ethnography of a Bring Your Own Device school. Learning, Media and Technology, 45(2), 193-207. 
Anderson, R. A., Crabtree, B. F., Steele, D. J., \& McDaniel, R. R. (2005). Case study research: The view from complexity science. Qualitative Health Research, 15(5), 669-685. https://doi.org/10.1177/1049732305275208

Armitage, A., \& Keeble-Allen, D. (2008). Undertaking a structured literature review or structuring a literature review: Tales from the field. Electronic Journal of Business Research Methods 6(2), 103-114.

Armstrong, A. (2014). Technology in the classroom: It's not a matter of'if,'but'when'and'how'. The Education Digest, 79(5), 39.

Arthur, W. B. (2009). The nature of technology: What it is and how it evolves. Simon and Schuster.

Baran, B., Yecan, E., Kaptan, B., \& Paşayiğit, O. (2020). Using augmented reality to teach fifth grade students about electrical circuits. Education and Information Technologies, 25, 1371-1385. https://doi.org/10.1007/s10639-019-10001-9

Baser, D., Ozden, M. Y., \& Karaarslan, H. (2017). Collaborative project-based learning: An integrative science and technological education project. Research in Science \& Technological Education, 35(2), 131-148. https://doi.org/10.1080/02635143.2016.1274723

Bates, A. T. (2018). Teaching in a digital age: Guidelines for designing teaching and learning (2nd ed.). BCcampus.

Biesta, G. (2007). Why "what works" won't work: Evidence-based practice and the democratic deficit in educational research. Educational Theory, 57(1), 1-22. https://doi.org/10.1111/j.17415446.2006.00241.x

Biesta, G. (2009). Good education in an age of measurement: On the need to reconnect with the question of purpose in education. Educational Assessment, Evaluation and Accountability, 21(1), 33-46. https://doi.org/10.1007/s11092-008-9064-9

Biesta, G. (2010). Why 'What works' still won't work: From evidence-based education to value-based education. Studies in Philosophy and Education, 29(5), 491-503.

https://doi.org/10.1007/s11217-010-9191-x

Biesta, G. (2015). Improving education through research? From effectiveness, causality and technology to purpose, complexity and culture. Policy Futures in Education, 14(2), 194-210. https://doi.org/10.1177/1478210315613900

Blaikie, N. (2007). Approaches to social enquiry: Advancing knowledge (2nd ed.). Polity.

Blaikie, N. (2009). Designing social research: The logic of anticipation (2nd ed.). Polity. 
Boulton, J. G., Allen, P. M., \& Bowman, C. (2015). Embracing complexity: Strategic perspectives for an age of turbulence. Oxford University Press. https://doi.org/10.1093/acprof:oso/9780199565252.001.0001

Boykin, A., Evmenova, A. S., Regan, K., \& Mastropieri, M. (2019). The impact of a computer-based graphic organizer with embedded self-regulated learning strategies on the argumentative writing of students in inclusive cross-curricula settings. Computers \& Education, 137, 78-90. https://doi.org/10.1016/i.compedu.2019.03.008

Braun, V., \& Clarke, V. (2006). Using thematic analysis in psychology. Qualitative Research in Psychology, 3(2), 77-101.

Bressler, D. M., Bodzin, A. M., \& Tutwiler, M. S. (2019). Engaging middle school students in scientific practice with a collaborative mobile game. Journal of Computer Assisted Learning, 35(2), 197-207. https://doi.org/10.1111/jcal.12321

Bryman, A. (2012). Social research methods. Oxford University Press.

Callon, M. (1987). Society in the making: the study of technology as a tool for sociological analysis. In W. E. Bijker, T. P. Hughes, \& T. Pinch (Eds.), The social construction of technological systems: New directions in the sociology and history of technology (pp. 83-103). MIT Press.

Callon, M. (2001). Actor Network Theory. In N. J. Smelser \& P. B. Baltes (Eds.), International encyclopedia of the social \& behavioral sciences (pp. 62-66). Pergamon. https://doi.org/https://doi.org/10.1016/B0-08-043076-7/03168-5

Camazine, S. (2001). Self-organization in biological systems. Princeton University Press.

Casti, J. L. (1995). Complexification : Explaining a paradoxical world through the science of surprise (1st ed.). HarperPerennial.

Cavus, N., \& Ibrahim, D. (2017). Learning English using children's stories in mobile devices. British Journal of Educational Technology, 48(2), 625-641.

Cerratto Pargman, T., Nouri, J., \& Milrad, M. (2018). Taking an instrumental genesis lens: New insights into collaborative mobile learning. British Journal of Educational Technology, 49(2), 219-234. https://doi.org/10.1111/bjet.12585

Chang, S.-C., \& Hwang, G.-J. (2018). Impacts of an augmented reality-based flipped learning guiding approach on students' scientific project performance and perceptions. Computers \& Education, 125, 226-239. https://doi.org/10.1016/i.compedu.2018.06.007 
Chang, S. C., Hsu, T. C., Kuo, W. C., \& Jong, M. S. Y. (2020). Effects of applying a VR-based two-tier test strategy to promote elementary students' learning performance in a Geology class. British Journal of Educational Technology, 51, 148-165. https://doi.org/10.1111/bjet.12790

Chauhan, S. (2017). A meta-analysis of the impact of technology on learning effectiveness of elementary students. Computers \& Education, 105, 14-30. https://doi.org/https://doi.org/10.1016/j.compedu.2016.11.005

Chee, K. N., Yahaya, N., Ibrahim, N. H., \& Hasan, M. N. (2017). Review of mobile learning trends 2010-2015: A meta-analysis. Journal of Educational Technology \& Society, 20(2), 113-126. http://www.jstor.org/stable/90002168

Cheng, S. C., Hwang, G. J., \& Chen, C. H. (2019). From reflective observation to active learning: A mobile experiential learning approach for environmental science education. British Journal of Educational Technology, 50(5), 2251-2270. https://doi.org/10.1111/bjet.12845

Cherner, T., \& Smith, D. (2016). Reconceptualizing TPACK to meet the needs of twenty-first-century education. The New Educator, 13(4), 329-349. https://doi.org/10.1080/1547688x.2015.1063744

Cilliers, P. (1998). Complexity and postmodernism: Understanding complex systems. Psychology Press.

Cilliers, P. (2001). Boundaries, hierarchies and networks in complex systems. International Journal of Innovation Management, 5(02), 135-147.

Cilliers, P. (2002). Complexity and postmodernism understanding complex systems. Routledge.

Claro, M., Nussbaum, M., López, X., \& Díaz, A. (2013). Introducing 1 to 1 in the classroom: A largescale experience in Chile. Journal of Educational Technology \& Society, 16(3), 315-328.

Click, A., \& Petit, J. (2010). Social networking and Web 2.0 in information literacy. The International Information \& Library Review, 42(2), 137-142.

Cochran-Smith, M., Ell, F., Grudnoff, L., Ludlow, L., Haigh, M., \& Hill, M. (2014). When Complexity Theory meets critical realism: A platform for research on initial teacher education. Teacher Education Quarterly, 41(1), 105-122.

Cohen, L., Manion, L., \& Morrison, K. (2017). Research methods in education (8th ed.). Routledge. https://doi.org/10.4324/9780203224342

Cordero, K., Nussbaum, M., Ibaseta, V., Otaíza, M. J., \& Chiuminatto, P. (2018). Read, write, touch: Co-construction and multiliteracies in a third-grade digital writing exercise. Journal of Computer Assisted Learning, 34(2), 162-173. https://doi.org/10.1111/jcal.12224 
Creswell, J. (2012). Educational research: Planning, conducting, and evaluating quantitative and qualitative research (4th ed.). Pearson.

Crompton, H., Burke, D., \& Gregory, K. H. (2017). The use of mobile learning in PK-12 education: A systematic review. Computers \& Education, 110, 51-63.

https://doi.org/10.1016/j.compedu.2017.03.013

Crompton, H., Burke, D., \& Lin, Y.-C. (2019). Mobile learning and student cognition: A systematic review of PK-12 research using Bloom's Taxonomy: Mobile learning \& student cognition. British Journal of Educational Technology, 50(2), 684-701. https://doi.org/10.1111/bjet.12674

Cui, J., \& Yu, S. (2019). Fostering deeper learning in a flipped classroom: Effects of knowledge graphs versus concept maps. British Journal of Educational Technology, 50(5), 2308-2328. https://doi.org/10.1111/bjet.12841

Cyr, A.-A., \& Anderson, N. D. (2015). Mistakes as stepping stones: Effects of errors on episodic memory among younger and older adults. Journal of Experimental Psychology: Learning, Memory, and Cognition, 41(3), 841.

Dahan Golan, D., Barzillai, M., \& Katzir, T. (2018). The effect of presentation mode on children's reading preferences, performance, and self-evaluations. Computers \& Education, 126, 346358. https://doi.org/10.1016/i.compedu.2018.08.001

Dahlström, H. (2019). Digital writing tools from the student perspective: Access, affordances, and agency. Education and Information Technologies, 24(2), 1563-1581.

https://doi.org/10.1007/s10639-018-9844-x

Daigneault, P.-M., Jacob, S., \& Ouimet, M. (2014). Using systematic review methods within a Ph. D. dissertation in political science: Challenges and lessons learned from practice. International Journal of Social Research Methodology, 17(3), 267-283.

Dasgupta, C., Magana, A. J., \& Vieira, C. (2019). Investigating the affordances of a CAD enabled learning environment for promoting integrated STEM learning. Computers \& Education, 129, 122-142. https://doi.org/10.1016/j.compedu.2018.10.014

Davis, B., \& Simmt, E. (2003). Understanding learning systems: Mathematics education and complexity science. Journal for Research in Mathematics Education, 137-167.

Davis, B., \& Sumara, D. (2001). Learning communities: Understanding the workplace as a complex system. New Directions for Adult and Continuing Education, 2001(92), 85-96.

Davis, B., \& Sumara, D. (2006). Complexity and education: Inquiries into learning, teaching, and research. Psychology Press. 
Davis, B., \& Sumara, D. (2010). 'If things were simple...': complexity in education. Journal of Evaluation in Clinical Practice, 16(4), 856-860. https://doi.org/10.1111/j.13652753.2010.01499.x

Davis, B., Sumara, D., \& Luce-Kapler, R. (2013). Engaging minds: Changing teaching in complex times. Routledge.

Debevc, M., Weiss, J., Šorgo, A., \& Kožuh, I. (2020). Solfeggio learning and the influence of a mobile application based on visual, auditory and tactile modalities. British Journal of Educational Technology, 51, 177-193. https://doi.org/10.1111/bjet.12792

Denzin, N. K. (1978). The research act : A theoretical introduction to sociological methods (2nd ed.). McGraw-Hill.

Dewey, J. (1998). The essential Dewey: Pragmatism, education, democracy (L. Hickman \& T. M. Alexander, Eds. Vol. 1). Indiana University Press.

Díaz, A., Nussbaum, M., \& Varela, I. (2015). Orchestrating the XO computer with digital and conventional resources to teach mathematics. Journal of Computer Assisted Learning, 31(3), 202-219. https://doi.org/10.1111/jcal.12081

Dindar, M., Kabakçı Yurdakul, I., \& Dönmez, F. I. (2015). Measuring cognitive load in test items: Static graphics versus animated graphics. Journal of Computer Assisted Learning, 31(2), 148-161. https://doi.org/10.1111/ical.12086

Ditzler, C., Hong, E., \& Strudler, N. (2016). How tablets are utilized in the classroom. Journal of Research on Technology in Education, 48(3), 181-193. https://doi.org/10.1080/15391523.2016.1172444

Dong, G., \& Potenza, M. N. (2015). Behavioural and brain responses related to Internet search and memory. European Journal of Neuroscience, 42(8), 2546-2554. https://doi.org/10.1111/ejn.13039

Dresang, E. T., \& Koh, K. (2009). Radical change theory, youth information behavior, and school libraries. Library Trends, 58(1), 26-50.

Ejsing-Duun, S., \& Skovbjerg, H. M. (2016). Copycat or creative innovator? Reproduction as a pedagogical strategy in schools. Electronic Journal of E-learning, 14(2), 83-93.

Ellis, R., \& Goodyear, P. (2013). Students' experiences of e-learning in higher education: The ecology of sustainable innovation. Routledge. 
Emmer, E. T., \& Stough, L. M. (2001). Classroom management: A critical part of educational psychology, with implications for teacher education. Educational Psychologist, 36(2), 103112. https://doi.org/10.1207/S15326985EP3602 5

Ennis, C. D. (1992). Reconceptualizing learning as a dynamical system. Journal of Curriculum and Supervision, 7(2), 115-130.

Expert Software Applications srl. (2018). Mindomo [Web app]. https://www.mindomo.com/

Fabian, K., Topping, K. J., \& Barron, I. G. (2018). Using mobile technologies for mathematics: Effects on student attitudes and achievement. Educational Technology Research and Development, 66(5), 1119-1139. https://doi.org/10.1007/s11423-018-9580-3

Falloon, G. (2015). What's the difference? Learning collaboratively using iPads in conventional classrooms. Computers \& Education, 84, 62-77.

https://doi.org/10.1016/i.compedu.2015.01.010

Falloon, G. (2017a). Exploring student thinking, problem solving, and collaboration in iPad-supported learning environments.

http://www.tlri.org.nz/sites/default/files/projects/TLRI\%20Summary\%20report\%20for\%20w ebsite\%20 Falloon.pdf

Falloon, G. (2017b). Mobile devices and apps as scaffolds to science learning in the primary classroom. Journal of Science Education and Technology, 26(6), 613-628.

https://doi.org/10.1007/s10956-017-9702-4

Fenwick, T. (2010). Re-thinking the "thing": Sociomaterial approaches to understanding and researching learning in work. Journal of Workplace Learning, 22(1/2), 104-116.

Fenwick, T., \& Edwards, R. (2010). Actor-Network Theory in education. Routledge.

Fenwick, T., Edwards, R., \& Sawchuk, P. (2015). Emerging approaches to educational research: Tracing the socio-material. Routledge.

Firth, J., Torous, J., Stubbs, B., Firth, J. A., Steiner, G. Z., Smith, L., Alvarez-Jimenez, M., Gleeson, J., Vancampfort, D., \& Armitage, C. J. (2019). The "online brain": How the Internet may be changing our cognition. World Psychiatry, 18(2), 119-129.

https://doi.org/10.1002/wps.20617

Freeman, B., Higgins, K. N., \& Horney, M. (2016). How students communicate mathematical ideas: An examination of multimodal writing using digital technologies. Contemporary Educational Technology, 7(4), 281-313. 
Froment, F., García González, A. J., \& Bohórquez, M. R. (2017). The use of social networks as a communication tool between teachers and students: A literature review. Turkish Online Journal of Educational Technology-TOJET, 16(4), 126-144.

Frydenberg, M. (2015). Achieving digital literacy through game development: An authentic learning experience. Interactive Technology and Smart Education, 12(4), 256-269.

Garneli, V., Giannakos, M., \& Chorianopoulos, K. (2017). Serious games as a malleable learning medium: The effects of narrative, gameplay, and making on students' performance and attitudes. British Journal of Educational Technology, 48(3), 842-859. https://doi.org/10.1111/bjet.12455

Geer, R., White, B., Zeegers, Y., Au, W., \& Barnes, A. (2017). Emerging pedagogies for the use of iPads in schools. British Journal of Educational Technology, 48(2), 490-498. https://doi.org/10.1111/bjet.12381

Ginier, S. (2018). SculptGL [Web app]. https://stephaneginier.com/sculptgl/

Girmen, P., Özkanal, Ü., \& Dayan, G. (2019). Digital storytelling in the language arts classroom. Universal Journal of Educational Research, 7(1), 55-65. https://doi.org/10.13189/ujer.2019.070108

Gough, D., Oliver, S., \& Thomas, J. (2012). An introduction to systematic reviews. Sage.

Grant, M. J., \& Booth, A. (2009). A typology of reviews: An analysis of 14 review types and associated methodologies. Health Information \& Libraries Journal, 26(2), 91-108.

Gresalfi, M. S., Rittle-Johnson, B., Loehr, A., \& Nichols, I. (2018). Design matters: Explorations of content and design in fraction games. Educational Technology Research and Development, 66(3), 579-596. https://doi.org/10.1007/s11423-017-9557-7

Gunbas, N. (2015). Students' mathematics word problem-solving achievement in a computer-based story: Word problem solving in a computer-story. Journal of Computer Assisted Learning, 31(1), 78-95. https://doi.org/10.1111/ical.12067

Habib, L., \& Wittek, L. (2007). The portfolio as artifact and actor. Mind, Culture, and Activity, 14(4), 266-282.

Hansen, A. K., McBeath, J. K., \& Harlow, D. B. (2019). No bones about it: How digital fabrication changes student perceptions of their role in the classroom. Journal of Pre-College Engineering Education Research (J-PEER), 9(1), 95-116. https://doi.org/10.7771/2157$\underline{9288.1155}$ 
Harper, B., \& Milman, N. B. (2016). One-to-one technology in K-12 classrooms: A review of the literature from 2004 through 2014. Journal of Research on Technology in Education, 48(2), 129-142.

Hartmann, S. B., Braae, L. Q. N., Pedersen, S., \& Khalid, M. (2017). The potentials of using cloud computing in schools: A systematic literature review. Turkish Online Journal of Educational Technology-TOJET, 16(1), 190-202.

Hashweh, M. Z. (2006). Teacher pedagogical constructions: A reconfiguration of pedagogical content knowledge. Teachers and Teaching, 11(3), 273-292. https://doi.org/10.1080/13450600500105502

Heinrich, C. J., Darling-Aduana, J., \& Martin, C. (2020). The potential and prerequisites of effective tablet integration in rural Kenya. British Journal of Educational Technology, 51(2), 498-514. https://doi.org/10.1111/bjet.12870

Holland, J. H. (1995). Hidden order: How adaptation builds complexity Addison-Wesley.

Hong, H.-Y., \& Lin, P.-Y. (2019). Elementary students enhancing their understanding of energy-saving through idea-centered collaborative knowledge-building scaffolds and activities. Educational Technology Research and Development, 67(1), 63-83. https://doi.org/10.1007/s11423-0189606-x

Hood, N. (2019). Manifestations of autonomy and control in a devolved schooling system: The case of New Zealand. On Education. Journal for Research and Debate, 2(5), 1-6. https://doi.org/10.17899/on ed.2019.5.6

Hsu, P. S., Van Dyke, M., Chen, Y., \& Smith, T. J. (2015). The effect of a graph-oriented computerassisted project-based learning environment on argumentation skills: Supporting argumentation. Journal of Computer Assisted Learning, 31(1), 32-58. https://doi.org/10.1111/jcal.12080

Hsu, P. S., Van Dyke, M., Chen, Y., \& Smith, T. J. (2016). A cross-cultural study of the effect of a graph-oriented computer-assisted project-based learning environment on middle school students' science knowledge and argumentation skills. Journal of Computer Assisted Learning, 32(1), 51-76. https://doi.org/10.1111/jcal.12118

Hurford, A. (2010). Complexity theories and theories of learning: Literature reviews and syntheses. In B. Sriraman \& L. English (Eds.), Theories on mathematics education: : Seeking new frontiers (pp. 567-589). Springer. https://doi.org/10.1007/978-3-642-00742-2 54

Hwang, G.-J., \& Chen, C.-H. (2017). Influences of an inquiry-based ubiquitous gaming design on students' learning achievements, motivation, behavioral patterns, and tendency towards critical thinking and problem solving: Inquiry-based ubiquitous game for students' learning. British Journal of Educational Technology, 48(4), 950-971.

https://doi.org/10.1111/bjet.12464 
Israel, M., Wherfel, Q. M., Shehab, S., Melvin, O., \& Lash, T. (2017). Describing elementary students' interactions in K-5 puzzle-based computer science environments using the collaborative computing observation instrument (C-COI). Proceedings of the 2017 ACM conference on international computing education research, Tacoma, Washington, USA. https://doi.org/10.1145/3105726.3106167

Iten, N., \& Petko, D. (2016). Learning with serious games: Is fun playing the game a predictor of learning success? British Journal of Educational Technology, 47(1), 151-163.

https://doi.org/10.1111/bjet.12226

Jaakkola, T., \& Veermans, K. (2015). Effects of abstract and concrete simulation elements on science learning. Journal of Computer Assisted Learning, 31(4), 300-313.

https://doi.org/10.1111/ical.12089

Jacobson, M. J., Kapur, M., \& Reimann, P. (2016). Conceptualizing debates in learning and educational research: Toward a complex systems conceptual framework of learning. Educational Psychologist, 51(2), 210-218. https://doi.org/10.1080/00461520.2016.1166963

Janssen, K. C., \& Phillipson, S. (2015). Are we ready for BYOD? An analysis of the implementation and communication of BYOD programs in Victorian schools. Australian Educational Computing, 30(2), 1-14.

Johnson, A. M., Ozogul, G., \& Reisslein, M. (2015). Supporting multimedia learning with visual signalling and animated pedagogical agent: Moderating effects of prior knowledge: Learning with signalling and APA. Journal of Computer Assisted Learning, 31(2), 97-115. https://doi.org/10.1111/jcal.12078

Joo-Nagata, J., Martinez Abad, F., García-Bermejo Giner, J., \& García-Peñalvo, F. J. (2017).

Augmented reality and pedestrian navigation through its implementation in m-learning and e-learning: Evaluation of an educational program in Chile. Computers \& Education, 111, 1-17. https://doi.org/10.1016/j.compedu.2017.04.003

Kang, J. H. (2019). Lonely collaborative information behavior (CIB) of youth. iConference 2019 Proceedings. http://hdl.handle.net/2142/103324,

$\mathrm{Ke}, \mathrm{F}$. (2019). Mathematical problem solving and learning in an architecture-themed epistemic game. Educational Technology Research and Development, 67(5), 1085-1104. https://doi.org/10.1007/s11423-018-09643-2

Kennedy, C., Rhoads, C., \& Leu, D. J. (2016). Online research and learning in science: A one-to-one laptop comparison in two states using performance based assessments. Computers \& Education, 100, 141-161. https://doi.org/10.1016/j.compedu.2016.05.003

Kiliçkaya, F. (2018). University language students' experience of handwriting and typing assignments: Issues and challenges. HyperCultura, 7, 1-12. 
Kim, E. (2013). Music technology-mediated teaching and learning approach for music education: A case study from an elementary school in South Korea. International Journal of Music Education, 31(4), 413-427. https://doi.org/10.1177/0255761413493369

Kim, H. J., \& Jang, H. Y. (2015). Factors influencing students' beliefs about the future in the context of tablet-based interactive classrooms. Computers \& Education, 89, 1-15.

https://doi.org/10.1016/i.compedu.2015.08.014

Kimbell-Lopez, K., Cummins, C., \& Manning, E. (2016). Developing digital literacy in the middle school classroom. Computers in the Schools, 33(4), 211-226. https://doi.org/10.1080/07380569.2016.1249731

Koehler, M. J., Mishra, P., \& Cain, W. (2013). What is technological pedagogical content knowledge (TPACK)? Journal of Education, 193(3), 13-19.

Koh, K. (2013). Adolescents' information-creating behavior embedded in digital Media practice using Scratch. Journal of the American Society for Information Science and Technology, 64(9), 1826-1841. https://doi.org/10.1002/asi.22878

Koh, K., Snead, J. T., \& Lu, K. (2019). The processes of maker learning and information behavior in a technology-rich high school class. Journal of the Association for Information Science and Technology, 70(12), 1395-1412. https://doi.org/10.1002/asi.24197

Krajcik, J. S., \& Shin, N. (2014). Project-Based Learning. In R. K. Sawyer (Ed.), The cambridge handbook of the learning sciences (2nd ed., pp. 275-297). Cambridge University Press.

Kuhn, L. (2007). Why utilize complexity principles in social inquiry? World Futures, 63(3-4), 156-175.

Kuiper, E., Volman, M., \& Terwel, J. (2005). The Web as an information resource in K-12 education: Strategies for supporting students in searching and processing information. Review of Educational Research, 75(3), 285-328.

Lai, C.-L., Hwang, G.-J., \& Tu, Y.-H. (2018). The effects of computer-supported self-regulation in science inquiry on learning outcomes, learning processes, and self-efficacy. Educational Technology Research and Development, 66(4), 863-892. https://doi.org/10.1007/s11423$\underline{018-9585-y}$

Lai, K.-W. (2018). The learner and the learning process: Research and practice in technologyenhanced learning. In J. Voogt, G. Knezek, R. Christensen, \& K.-W. Lai (Eds.), Second handbook of information technology in primary and secondary education (pp. 127-142). Springer https://doi.org/10.1007/978-3-319-71054-9 8

Lange, A. A. (2019). Technology, instructional methods, and the systemic messiness of innovation: Improving reading fluency for low socio-economic elementary school students. Educational 
Technology Research and Development, 67(5), 1333-1350. https://doi.org/10.1007/s11423$\underline{019-09675-2}$

Lanning, S., \& Turner, R. (2010). Trends in print vs. electronic use in school libraries. The Reference Librarian, 51(3), 212-221. https://doi.org/10.1080/02763871003800601

Latour, B. (1987). Science in action: How to follow scientists and engineers through society. Harvard University Press.

Latour, B. (1999). On recalling ANT. The Sociological Review, 47(S1), 15-25.

Latour, B. (2005). Reassembling the social: An introduction to actor-network-theory. Oxford University Press.

Law, J. (1984). On the methods of long-distance control: vessels, navigation and the Portuguese route to India. The Sociological Review, 32(S1), 234-263.

Law, J. (1999). After ANT: Complexity, naming and topology. The Sociological Review, 47(S1), 1-14.

Law, J. (2002). Objects and spaces. Theory, Culture \& Society, 19(5-6), 91-105.

https://doi.org/doi:10.1177/026327602761899165

Law, J. (2009). Actor Network Theory and material semiotics. The New Blackwell Companion to Social Theory, 3, 141-158.

Law, J., \& Callon, M. (1988). Engineering and sociology in a military aircraft project: A network analysis of technological change. Social Problems, 35(3), 284-297.

Lei, J., \& Zhao, Y. (2008). One-to-one computing: What does it bring to schools? Journal of Educational Computing Research, 39(2), 97-122.

Levy, Y., \& Ellis, T. J. (2006). A systems approach to conduct an effective literature review in support of information systems research. Informing Science Journal 9, 181-211.

Liao, C., Motter, J. L., \& Patton, R. M. (2016). Tech-savvy girls: Learning 21st-century skills through STEAM digital artmaking. Art Education, 69(4), 29-35. https://doi.org/10.1080/00043125.2016.1176492

Liao, C. C. Y., Chang, W.-C., \& Chan, T.-W. (2018). The effects of participation, performance, and interest in a game-based writing environment. Journal of Computer Assisted Learning, 34(3), 211-222. https://doi.org/10.1111/jcal.12233 
Limberg, L., \& Alexandersson, M. (2017). Learning and information seeking. In J. D. McDonald \& M. Levine-Clark (Eds.), Encyclopedia of library and information sciences (4th ed., pp. 2751-2761). CRC Press. https://doi.org/10.1081/E-ELIS4

Lin, C. H., Chiu, C. H., Hsu, C. C., Wang, T. I., \& Chen, C. H. (2018). The effects of computerized inquiry-stage-dependent argumentation assistance on elementary students' science process and argument construction skills. Journal of Computer Assisted Learning, 34(3), 279-292. https://doi.org/10.1111/jcal.12241

Lincoln, Y.-G., \& Guba, E. (1985). Naturalistic inquiry. Sage.

Lips, M., Eppel, E., McRae, H., Starkey, L., Sylvester, A., Parore, P., \& Barlow, L. (2017). Understanding children's use and experience with digital technologies Final research report.

Liu, C.-C., Yang, C.-Y., \& Chao, P.-Y. (2019). A longitudinal analysis of student participation in a digital collaborative storytelling activity. Educational Technology Research and Development, 67(4), 907-929. https://doi.org/10.1007/s11423-019-09666-3

Liu, M.-C., Huang, Y.-M., \& Xu, Y.-H. (2018). Effects of individual versus group work on learner autonomy and emotion in digital storytelling. Educational Technology Research and Development, 66(4), 1009-1028. https://doi.org/10.1007/s11423-018-9601-2

Liu, Y., \& Gu, X. (2020). Media multitasking, attention, and comprehension: A deep investigation into fragmented reading. Educational Technology Research and Development, 68, 67-87. https://doi.org/10.1007/s11423-019-09667-2

Lofthus, L., \& Silseth, K. (2019). Students choosing digital sources: Studying students' information literacy in group work with tablets. E-Learning and Digital Media, 16(4), 284-300. https://doi.org/10.1177/2042753019835882

Loong, E. Y.-K., \& Herbert, S. (2018). Primary school teachers' use of digital technology in mathematics: The complexities. Mathematics Education Research Journal, 30, 1-24. https://doi.org/10.1007/s13394-018-0235-9

Luck, J. (2008). Lost in translations: A socio-technical study of interactive videoconferencing at an Australian University (Unpublished PhD thesis). Central Queensland University, Queensland, Australia.

Luck, J. (2013). Actor-network theory: A device for reimagining participants in education research. In W. Midgley, P. A. Danaher, \& M. Baguley (Eds.), The role of participants in education research: Ethics, epistemologies, and methods (pp. 226-240). Routledge.

Malliari, A., Togia, A., Korobili, S., \& Nitsos, I. (2017). Information literacy skills of Greek high-school students: Results of an empirical survey. Qualitative and Quantitative Methods in Libraries, 3(1), 271-281. 
Masek, M., Boston, J., Lam, C. P., \& Corcoran, S. (2017). Improving mastery of fractions by blending video games into the Math classroom: Game enhanced fractions learning. Journal of Computer Assisted Learning, 33(5), 486-499. https://doi.org/10.1111/ical.12194

Mason, L., Scrimin, S., Tornatora, M. C., Suitner, C., \& Moè, A. (2018). Internet source evaluation: The role of implicit associations and psychophysiological self-regulation. Computers \& Education, 119, 59-75. https://doi.org/10.1016/j.compedu.2017.12.009

McDaniel, R. R., Jordan, E. M., \& Fleeman, F. B. (2003). Surprise, surprise, surprise! A complexity science view of the unexpected. Health Care Management Review, 28(3), 266-278. https://doi.org/10.1097/00004010-200307000-00008

McFarlane, A. E. (2019). Devices and desires: Competing visions of a good education in the digital age. British Journal of Educational Technology, 50(3), 1125-1136.

https://doi.org/10.1111/bjet.12764

McRae, H., McKenzie, T., \& Toia, R. (2010). ICT tools supporting teacher practice in language acquisition. Ubiquitous Learning: An International Journal, 2(3), 81-92.

Melander Bowden, H. (2019). Problem-solving in collaborative game design practices: Epistemic stance, affect, and engagement. Learning, Media and Technology, 44(2), 124-143. https://doi.org/10.1080/17439884.2018.1563106

Merriam, S. B., \& Tisdell, E. J. (2015). Qualitative research: A guide to design and implementation (4th ed.). Wiley.

Miller, B. W., Cromley, J. G., \& Newcombe, N. S. (2016). Improving diagrammatic reasoning in middle school science using conventions of diagrams instruction: Improving diagrammatic reasoning. Journal of Computer Assisted Learning, 32(4), 374-390. https://doi.org/10.1111/jcal.12143

Ministry of Education. (2007). The New Zealand curriculum. Learning Media.

Mitchell, M. (2009). Complexity: A guided tour. Oxford University Press.

Mouza, C., \& Barrett-Greenly, T. (2015). Bridging the app gap: An examination of a professional development initiative on mobile learning in urban schools. Computers \& Education, 88, 114. https://doi.org/10.1016/i.compedu.2015.04.009

Ng, O.-L., \& Chan, T. (2019). Learning as making: Using 3D computer-aided design to enhance the learning of shape and space in STEM-integrated ways. British Journal of Educational Technology, 50(1), 294-308. https://doi.org/10.1111/bjet.12643 
Nogry, S., \& Varly, P. (2017). Everyday laptop use by children in a southern country: A mixed-method approach. Journal of Research on Technology in Education, 50(1), 18-33.

https://doi.org/10.1080/15391523.2017.1388200

Nowell, L. S., Norris, J. M., White, D. E., \& Moules, N. J. (2017). Thematic analysis: Striving to meet the trustworthiness criteria. International Journal of Qualitative Methods, 16(1), 1-13.

https://doi.org/10.1177/1609406917733847

Ong, B. K. (2010). Abductive research strategy (ARS) and the generation of grounded concepts: Two empirical examples. International Journal of Qualitative Methods, 9(4), 454-454.

Ottestad, G., \& Gudmundsdottir, G. B. (2018). Information and communication technology policy in primary and secondary education in Europe. In J. Voogt, G. Knezek, R. Christensen, \& K.-W. Lai (Eds.), Second handbook of information technology in primary and secondary education (pp. 1343-1362). Springer https://doi.org/10.1007/978-3-319-71054-9 92

Park, Y., Xu, Y., Collins, P., Farkas, G., \& Warschauer, M. (2019). Scaffolding learning of language structures with visual-syntactic text formatting. British Journal of Educational Technology, 50(4), 1896-1912. https://doi.org/10.1111/bjet.12689

Patton, M. Q. (2011). Developmental evaluation: Applying complexity concepts to enhance innovation and use. Guilford Press.

Patton, M. Q. (2015). Qualitative research \& evaluation methods: integrating theory and practice (4th ed.). SAGE

Patton, Q. M. (2002). Qualitative research and evaluation methods (3rd ed.). Sage Publications Inc.

Perry, A., \& Hammond, N. (2002). Systematic reviews: The experiences of a PhD student. Psychology Learning \& Teaching, 2(1), 32-35.

Porat, E., Blau, I., \& Barak, A. (2018). Measuring digital literacies: Junior high-school students' perceived competencies versus actual performance. Computers \& Education, 126, 23-36. https://doi.org/10.1016/i.compedu.2018.06.030

Powell, M. A., Fitzgerald, R. M., Taylor, N., \& Graham, A. (2012). International literature review: Ethical issues in undertaking research with children and young people. https://www.researchgate.net/profile/Mary Ann Powell2/publication/238601826 Internat ional literature review ethical issues in undertaking research with children and young people/links/00b4953427bf5e0d53000000/International-literature-review-ethical-issuesin-undertaking-research-with-children-and-young-people.pdf

Price, S., Jewitt, C., \& Sakr, M. (2016). Embodied experiences of place: A study of history learning with mobile technologies. Journal of Computer Assisted Learning, 32(4), 345-359.

https://doi.org/10.1111/jcal.12137 
Priestley, M., \& Sinnema, C. (2014). Downgraded curriculum? An analysis of knowledge in new curricula in Scotland and New Zealand. Curriculum Journal, 25(1), 50-75. https://doi.org/10.1080/09585176.2013.872047

Puentedura, R. R. (2009). SAMR: A contextualized introduction. http://hippasus.com/rrpweblog/archives/2014/01/15/SAMRABriefContextualizedlntroducti on.pdf

Punie, Y., Zinnbauer, D., \& Cabrera, M. (2006). A review of the impact of ICT on learning. https://citeseerx.ist.psu.edu/viewdoc/download?doi=10.1.1.463.9921\&rep=rep1\&type=pdf

Radi, O. B. (2015). Studies relating to computer use of spelling and grammar checkers and educational achievement. 12th International Conference on Cognition and Exploratory Learning in Digital Age (CELDA 2015), 163-172. https://files.eric.ed.gov/fulltext/ED562194.pdf

Roth, W.-M. (1996). Knowledge diffusion in a grade 4-5 classroom during a unit on civil engineering: An analysis of a classroom community in terms of its changing resources and practices. Cognition and Instruction, 14(2), 179-220.

Sayes, E. (2014). Actor-Network Theory and methodology: Just what does it mean to say that nonhumans have agency? Social Studies of Science, 44(1), 134-149.

Schmitt, L. J., \& Weinberger, A. (2019, 2019/06//). Fourth graders' dyadic learning on multi-touch interfaces-versatile effects of verbalization prompts. Educational Technology Research and Development, 67(3), 519-539. https://doi.org/10.1007/s11423-018-9619-5

Scholz, C. (2016). A study of the application of a Bring Your Own Device strategy in an elementary school (Unpublished PhD thesis). Walden University, Minnesota, USA.

Schwab, K. (2016). The global competitiveness report 2016-2017, ed. WE Forum,

SCImago. (n.d.). SJR - SCImago Journal \& Country Rank [Portal]. http://www.scimagojr.com

Serdyukov, P. (2017). Innovation in education: What works, what doesn't, and what to do about it? Journal of Research in Innovative Teaching \& Learning, 10(1), 4-33. https://doi.org/10.1108/JRIT-10-2016-0007

Severin, E., \& Capota, C. (2011). One-to-one laptop programs in Latin America and the Caribbean. https://publications.iadb.org/publications/english/document/One-to-One-Laptop-Programsin-Latin-America-and-the-Caribbean-Panorama-and-Perspectives.pdf 
Shadiev, R., Hwang, W. Y., Huang, Y. M., \& Liu, T. Y. (2018). Facilitating application of language skills in authentic environments with a mobile learning system. Journal of Computer Assisted Learning, 34(1), 42-52. https://doi.org/10.1111/jcal.12212

Slavin, R. E. (2002). Evidence-based education policies: Transforming educational practice and research. Educational Researcher, 31(7), 15-21. https://doi.org/10.3102/0013189X031007015

Song, Y. (2018). Improving primary students' collaborative problem solving competency in projectbased science learning with productive failure instructional design in a seamless learning environment. Educational Technology Research and Development, 66(4), 979-1008. https://doi.org/10.1007/s11423-018-9600-3

Sørensen, E. (2009). The materiality of learning: Technology and knowledge in educational practice. Cambridge University Press.

Spante, M. (2019). Digital creativity: Learning by story driven digital production. International Journal of Information and Learning Technology, 36(3), 182-191. https://doi.org/10.1108/ijilt-11$\underline{2018-0129}$

Sparrow, B., Liu, J., \& Wegner, D. M. (2011). Google effects on memory: Cognitive consequences of having information at our fingertips. Science, 333(6043), 776-778.

Spotify USA Inc. (2018). SoundTrap [Web app]. https://www.soundtrap.com/

Starkey, L. (2010). Digital saviours: Digitally able secondary school teachers in their first year of teaching (Unpublished PhD thesis). Victoria University of Wellington, Wellington, New Zealand.

Starkey, L. (2012). Teaching and learning in the digital age. Routledge.

Starkey, L., Eppel, E. A., \& Sylvester, A. (2018). How do 10-year-old New Zealanders participate in a digital world? Information, Communication \& Society, 22(13), 1929-1944.

https://doi.org/10.1080/1369118x.2018.1472795

Starkey, L., \& Finger, G. (2018). Information and communication technology in educational policies in Australia and New Zealand. In J. Voogt, G. Knezek, R. Christensen, \& K.-W. Lai (Eds.), Second handbook of information technology in primary and secondary education (pp. 1-20). Springer https://doi.org/10.1007/978-3-319-53803-7 87-2

Starkey, L., Sylvester, A., \& Johnstone, D. (2017). Negotiating digital divides: Perspectives from the New Zealand schooling system. Journal of Research on Technology in Education, 49(1-2), 3142. 
Starkey, L., \& Zhong, J. (2019). The effect of netbook ownership on children's academic achievement in mathematics, reading, and writing. Journal of Computer Assisted Learning, 35(1), 34-41. https://doi.org/10.1111/jcal.12308

Stebner, F., Kühl, T., Höffler, T. N., Wirth, J., \& Ayres, P. (2017). The role of process information in narrations while learning with animations and static pictures. Computers \& Education, 104, 34-48. https://doi.org/10.1016/j.compedu.2016.11.001

Stenliden, L. (2014). Visual storytelling interacting in school: Learning conditions in the social science classroom (Unpublished PhD thesis). Linköping University, Linköping, Sweden.

Storm, B. C., \& Stone, S. M. (2015). Saving-enhanced memory: The benefits of saving on the learning and remembering of new information. Psychol Sci, 26(2), 182-188. https://doi.org/10.1177/0956797614559285

Storm, B. C., Stone, S. M., \& Benjamin, A. S. (2017). Using the Internet to access information inflates future use of the Internet to access other information. Memory, 25(6), 717-723. https://doi.org/10.1080/09658211.2016.1210171

Stoyle, K. L., \& Morris, B. J. (2017). Blogging mathematics: Using technology to support mathematical explanations for learning fractions. Computers \& Education, 111, 114-127. https://doi.org/10.1016/i.compedu.2017.04.007

Sturman, L., \& Sizmur, J. (2011). International comparison of computing in schools. https://www.nfer.ac.uk/publications/cis101/cis101.pdf

Sullivan, F. R., \& Heffernan, J. (2016). Robotic construction kits as computational manipulatives for learning in the STEM disciplines. Journal of Research on Technology in Education, 48(2), 105128.

Sung, Y.-T., Chang, K.-E., \& Liu, T.-C. (2016). The effects of integrating mobile devices with teaching and learning on students' learning performance: A meta-analysis and research synthesis. Computers \& Education, 94, 252-275. https://doi.org/10.1016/i.compedu.2015.11.008

Tan, S. C., Cheah, H. M., Chen, W., \& Choy, D. (2017). Pushing the frontier: A cohesive system-wide approach to integrating ICT into education. Springer.

Thiese, M. S. (2014). Observational and interventional study design types: An overview. Biochemia Medica, 24(2), 199-210. https://doi.org/10.11613/BM.2014.022

Tho, S. W., \& Yeung, Y. Y. (2018). An implementation of remote laboratory for secondary science education. Journal of Computer Assisted Learning, 34(5), 629-640.

https://doi.org/10.1111/jcal.12273 
Tingir, S., Cavlazoglu, B., Caliskan, O., Koklu, O., \& Intepe-Tingir, S. (2017). Effects of mobile devices on K-12 students' achievement: A meta-analysis. Journal of Computer Assisted Learning, 33(4), 355-369. https://doi.org/10.1111/jcal.12184

Tirado-Morueta, R., Berlanga-Fernández, I., Vales-Villamarín, H., Guzmán Franco, M. D., DuarteHueros, A., \& Aguaded-Gómez, J. I. (2020). Study of a sequence to stimulate the engagement in one-to-one iPad programs at elementary schools. Education and Information Technologies, 25, 509-532. https://doi.org/10.1007/s10639-019-09974-4

Traxler, J. M., \& Crompton, H. (2015). Mobile learning. In Encyclopedia of mobile phone behavior (pp. 506-518). IGI Global. https://doi.org/10.4018/978-1-4666-8239-9.ch042

Usher, R., \& Edwards, R. (2007). Lifelong learning-signs, discourses, practices. Springer

Uzun, A. M., \& Yıldırım, Z. (2018). Exploring the effect of using different levels of emotional design features in multimedia science learning. Computers \& Education, 119, 112-128.

https://doi.org/10.1016/j.compedu.2018.01.002

van der Meij, H., \& van der Meij, J. (2016). The effects of reviews in video tutorials. Journal of Computer Assisted Learning, 32(4), 332-344. https://doi.org/10.1111/ical.12136

van Weerdenburg, M., Tesselhof, M., \& van der Meijden, H. (2019). Touch-typing for better spelling and narrative-writing skills on the computer. Journal of Computer Assisted Learning, 35(1), 143-152. https://doi.org/10.1111/jcal.12323

Varier, D., Dumke, E. K., Abrams, L. M., Conklin, S. B., Barnes, J. S., \& Hoover, N. R. (2017). Potential of one-to-one technologies in the classroom: Teachers and students weigh in. Educational Technology Research and Development, 65(4), 967-992. https://doi.org/10.1007/s11423$\underline{017-9509-2}$

Walton, G., Childs, M., \& Jugo, G. (2019). The creation of digital artefacts as a mechanism to engage students in studying literature. British Journal of Educational Technology, 50(3), 1060-1086. https://doi.org/10.1111/bjet.12785

Waltz, S. B. (2006). Nonhumans unbound: Actor-network theory and the reconsideration of "things" in educational foundations. The Journal of Educational Foundations, 20(3/4), 51.

Wang, H. (2019). Wuwei, self-organization, and classroom dynamics. Educational Philosophy and Theory, 51(11), 1141-1151. https://doi.org/10.1080/00131857.2018.1564661

Wang, Y., Wu, L., Luo, L., Zhang, Y., \& Dong, G. (2017). Short-term Internet search using makes people rely on search engines when facing unknown issues. PLoS One, 12(4), e0176325. https://doi.org/10.1371/journal.pone.0176325 
Wegner, D. M. (1987). Transactive memory: A contemporary analysis of the group mind. In B. Mullen \& G. R. Goethals (Eds.), Theories of group behavior (pp. 185-208). Springer. https://doi.org/10.1007/978-1-4612-4634-3 9

Wetzel, S., Bertel, S., Montag, M., \& Zander, S. (2020). Spatial task solving on tablets: Analysing mental and physical rotation processes of 12-13-year olds. Educational Technology Research and Development, 68, 363-381. https://doi.org/10.1007/s11423-019-09699-8

Wilson, J., \& Czik, A. (2016). Automated essay evaluation software in English Language Arts classrooms: Effects on teacher feedback, student motivation, and writing quality. Computers \& Education, 100, 94-109. https://doi.org/10.1016/i.compedu.2016.05.004

Witherspoon, E. B., Schunn, C. D., Higashi, R. M., \& Shoop, R. (2018). Attending to structural programming features predicts differences in learning and motivation. Journal of Computer Assisted Learning, 34(2), 115-128. https://doi.org/10.1111/jcal.12219

Yelland, N. J. (2018). A pedagogy of multiliteracies: Young children and multimodal learning with tablets. British Journal of Educational Technology, 49(5), 847-858. https://doi.org/10.1111/bjet.12635

Yildirim, G. (2018). Opinions of secondary school students on 3D modelling programs and 3D printers according to using experiences. Turkish Online Journal of Educational Technology-TOJET, 17(4), 19-31.

Yildiz Durak, H. (2018). Digital story design activities used for teaching programming effect on learning of programming concepts, programming self-efficacy, and participation and analysis of student experiences. Journal of Computer Assisted Learning, 34(6), 740-752. https://doi.org/10.1111/jcal.12281

Yin, R. K. (2009). Case study research: Design and methods (4th ed.). SAGE.

Young, K. A. (2005). Direct from the source: the value of 'think aloud' data in understanding learning. The Journal of Educational Enquiry, 6(1), 19 - 33. http://hdl.handle.net/10453/6348

Zacharia, Z. C., Lazaridou, C., \& Avraamidou, L. (2016). The use of mobile devices as means of data collection in supporting elementary school students' conceptual understanding about plants. International Journal of Science Education, 38(4), 596-620.

https://doi.org/10.1080/09500693.2016.1153811

Zheng, B., Arada, K., Niiya, M., \& Warschauer, M. (2014). One-to-one laptops in K-12 classrooms: Voices of students. Pedagogies: An International Journal, 9(4), 279-299.

Zilka, G. C. (2020). Teenagers connected to digital environments - what happens when they get to school? Commonalities, similarities and differences from their perspective. Education and Information Technologies, 25, 1743-1758. https://doi.org/10.1007/s10639-019-10052-y 


\section{Appendices}

\section{Appendix A: Table of Systematised Review Studies}

\begin{tabular}{|c|c|c|c|c|c|c|c|}
\hline & & Author & Country & Sample & $\begin{array}{l}\text { Level, } \\
\text { age }\end{array}$ & Device type & Methods \\
\hline 1 & El1 S & Baran et al. (2020) & Turkey & 30 & $\begin{array}{c}\text { Grade } \\
5\end{array}$ & Tablet & Mixed \\
\hline 2 & E12 L & Dahlström (2019) & Sweden & 99 & $\begin{array}{l}\text { Age } \\
10-12\end{array}$ & $\begin{array}{l}\text { Computer } \\
\text { Tablet }\end{array}$ & Mixed \\
\hline 3 & EI3 LM & $\begin{array}{l}\text { Tirado-Morueta et al. } \\
\text { (2020) }\end{array}$ & Spain & 1011 & $\begin{array}{c}\text { Grades } \\
5-6\end{array}$ & iPad & Quantitative \\
\hline 4 & EI4 G & Zilka (2020) & Israel & 233 & $\begin{array}{l}\text { Age } \\
13-18\end{array}$ & $\begin{array}{l}\text { Smartphone } \\
\text { Computer }\end{array}$ & Mixed \\
\hline 5 & CE1 SH & Boykin et al. (2019) & USA & 69 & $\begin{array}{c}\text { Grades } \\
4-6\end{array}$ & Laptop & Quantitative \\
\hline 6 & CE2 SD & $\begin{array}{l}\text { Chang and Hwang } \\
\text { (2018) }\end{array}$ & $\begin{array}{c}\text { Taiwan } \\
\text { China }\end{array}$ & 111 & $\begin{array}{c}\text { Grade } \\
5\end{array}$ & Tablet & Mixed \\
\hline 7 & CE3 L & $\begin{array}{l}\text { Dahan Golan et al. } \\
\text { (2018) }\end{array}$ & Israel & 82 & $\begin{array}{c}\text { Grades } \\
5-6\end{array}$ & Computer & Quantitative \\
\hline 8 & CE4 T & $\begin{array}{l}\text { Dasgupta et al. } \\
\text { (2019) }\end{array}$ & USA & 408 & $\begin{array}{c}\text { Grades } \\
6-8\end{array}$ & Laptop & Quantitative \\
\hline 9 & CE5 $\mathrm{H}$ & $\begin{array}{l}\text { Joo-Nagata et al. } \\
(2017)\end{array}$ & Chile & 143 & $\begin{array}{l}\text { Age } \\
12-14\end{array}$ & $\begin{array}{l}\text { iPad } \\
\text { PC }\end{array}$ & Mixed \\
\hline 10 & CE6 S & $\begin{array}{l}\text { Kennedy et al. } \\
\text { (2016) }\end{array}$ & USA & 1129 & $\begin{array}{c}\text { Grade } \\
7\end{array}$ & Laptop & Quantitative \\
\hline 11 & CE7 G & Kim and Jang (2015) & South Korea & 277 & $\begin{array}{c}\text { Grades } \\
4-6\end{array}$ & Tablet & Quantitative \\
\hline 12 & CE8 EMH & $\begin{array}{l}\text { Mouza and Barrett- } \\
\text { Greenly (2015) }\end{array}$ & USA & 46 & $\begin{array}{l}\text { Grade } \\
5\end{array}$ & iPad & Qualitative \\
\hline 13 & CE9 D & Porat et al. (2018) & Israel & 280 & $\begin{array}{c}\text { Grade } \\
7\end{array}$ & $\begin{array}{l}\text { Computer } \\
\text { Laptop }\end{array}$ & Quantitative \\
\hline 14 & CE10 S & Stebner et al. (2017) & Germany & 464 & $\begin{array}{c}\text { Grades } \\
7-8\end{array}$ & Laptop & Quantitative \\
\hline 15 & CE11 M & $\begin{array}{l}\text { Stoyle and Morris } \\
\text { (2017) }\end{array}$ & USA & 134 & $\begin{array}{c}\text { Grade } \\
5\end{array}$ & Computer & Quantitative \\
\hline 16 & CE12 S & $\begin{array}{l}\text { Uzun and Yildırım } \\
\text { (2018) }\end{array}$ & Turkey & 113 & $\begin{array}{c}\text { Grade } \\
\quad 7\end{array}$ & Computer & Quantitative \\
\hline 17 & CE13 L & $\begin{array}{l}\text { Wilson and Czik } \\
\text { (2016) }\end{array}$ & USA & 145 & $\begin{array}{l}\text { Grade } \\
\quad 8\end{array}$ & Chromebook & Quantitative \\
\hline 18 & BJ1 M & $\begin{array}{l}\text { Alcoholado et al. } \\
\text { (2016) }\end{array}$ & Chile & 81 & $\begin{array}{c}\text { Grade } \\
3\end{array}$ & $\begin{array}{l}\text { Computer } \\
\text { Netbook }\end{array}$ & Mixed \\
\hline 19 & BJ2 LSM & $\begin{array}{l}\text { Cerratto Pargman et } \\
\text { al. (2018) }\end{array}$ & Sweden & 600 & $\begin{array}{c}\text { Grades } \\
4-8\end{array}$ & Tablet & Qualitative \\
\hline
\end{tabular}




\begin{tabular}{|c|c|c|c|c|c|c|c|}
\hline 20 & BJ3 S & Chang et al. (2020) & $\begin{array}{l}\text { Hong Kong } \\
\text { China }\end{array}$ & 42 & $\begin{array}{l}\text { Grade } \\
5\end{array}$ & Tablet & Mixed \\
\hline 21 & BJ4 S & Cheng et al. (2019) & $\begin{array}{l}\text { Taiwan } \\
\text { China }\end{array}$ & 50 & $\begin{array}{c}\text { Grade } \\
4\end{array}$ & Tablets & Mixed \\
\hline 22 & BJ5 L & Cui and Yu (2019) & China & 74 & $\begin{array}{l}\text { Grade } \\
5\end{array}$ & Laptop & Quantitative \\
\hline 23 & BJ6 U & Debevc et al. (2020) & Slovenia & 42 & $\begin{array}{l}\text { Age } \\
9-13\end{array}$ & $\begin{array}{c}\text { Computer } \\
\text { Smartphone } \\
\text { Tablet }\end{array}$ & Quantitative \\
\hline 24 & BJ7 T & Garneli et al. (2017) & Greece & 80 & $\begin{array}{l}\text { Age } \\
13\end{array}$ & Computer & Mixed \\
\hline 25 & BJ8 G & Geer et al. (2017) & Australia & 267 & $\begin{array}{l}\text { Year } \\
4-9\end{array}$ & iPads & Mixed \\
\hline 26 & BJ9 L & Heinrich et al. (2020) & Kenya & 253 & $\begin{array}{c}\text { Age } \\
\mu=12.6\end{array}$ & Tablets & Mixed \\
\hline 27 & BJ10 S & $\begin{array}{l}\text { Hwang and Chen } \\
\text { (2017) }\end{array}$ & $\begin{array}{l}\text { Taiwan } \\
\text { China }\end{array}$ & 101 & $\begin{array}{c}\text { Grade } \\
6\end{array}$ & Tablet & Quantitative \\
\hline 28 & BJ11 E & $\begin{array}{l}\text { Iten and Petko } \\
\text { (2016) }\end{array}$ & Switzerland & 74 & $\begin{array}{l}\text { Age } \\
10-13\end{array}$ & Notebook & Quantitative \\
\hline 29 & BJ12 T & $\mathrm{Ng}$ and Chan (2019) & $\begin{array}{l}\text { Hong Kong } \\
\text { China }\end{array}$ & 165 & $\begin{array}{l}\text { Age } \\
10-13\end{array}$ & $\begin{array}{l}\text { Computer } \\
\text { iPads }\end{array}$ & Qualitative \\
\hline 30 & BJ13 L & Park et al. (2019) & USA & 1085 & $\begin{array}{c}\text { Grades } \\
4-6\end{array}$ & Laptops & Mixed \\
\hline 31 & BJ14 L & Walton et al. (2019) & $\begin{array}{c}\text { Croatia } \\
\text { Denmark } \\
\text { Poland } \\
\text { Sweden } \\
\text { UK } \\
\end{array}$ & 400 & $\begin{array}{l}\text { Age } \\
9-15\end{array}$ & Computer & Mixed \\
\hline 32 & ET1 ML & Ale et al. (2017) & India & 205 & $\begin{array}{l}\text { Grades } \\
4-5\end{array}$ & Laptop & Quantitative \\
\hline 33 & ET2 M & Fabian et al. (2018) & Scotland & 52 & $\begin{array}{c}\text { Grades } \\
6-7\end{array}$ & Tablet & Mixed \\
\hline 34 & ET3 M & Gresalfi et al. (2018) & USA & 95 & $\begin{array}{c}\text { Grade } \\
3\end{array}$ & iPad & Mixed \\
\hline 35 & ET4 S & Hong and Lin (2019) & $\begin{array}{l}\text { Taiwan } \\
\text { China }\end{array}$ & 34 & $\begin{array}{l}\text { Grade } \\
5\end{array}$ & Laptop & Mixed \\
\hline 36 & ET5 M & $\operatorname{Ke}(2019)$ & USA & 61 & $\begin{array}{l}\text { Grade } \\
\quad 6\end{array}$ & Laptop & Mixed \\
\hline 37 & ET6 S & Lai et al. (2018) & $\begin{array}{l}\text { Taiwan } \\
\text { China }\end{array}$ & 56 & $\begin{array}{c}\text { Grade } \\
4\end{array}$ & $\begin{array}{l}\text { Mobile } \\
\text { devices }\end{array}$ & Mixed \\
\hline 38 & ET7 L & Lange (2019) & USA & 98 & $\begin{array}{c}\text { Grade } \\
3\end{array}$ & Computer & Mixed \\
\hline 39 & ET8 L & Liu et al. (2019) & $\begin{array}{l}\text { Taiwan } \\
\text { China }\end{array}$ & 26 & $\begin{array}{c}\text { Grade } \\
3\end{array}$ & $\mathrm{iPad}$ & Mixed \\
\hline
\end{tabular}




\begin{tabular}{|c|c|c|c|c|c|c|c|}
\hline 40 & ET9 L & Liu et al. (2018) & $\begin{array}{l}\text { Taiwan } \\
\text { China }\end{array}$ & 55 & $\begin{array}{l}\text { Grade } \\
\quad 6\end{array}$ & Tablet & Quantitative \\
\hline 41 & ET10 L & Liu and Gu (2020) & China & 129 & $\begin{array}{c}\text { Grade } \\
5\end{array}$ & Computer & Quantitative \\
\hline 42 & ET11 M & $\begin{array}{l}\text { Schmitt and } \\
\text { Weinberger (2019) }\end{array}$ & Germany & 162 & $\begin{array}{c}\text { Grade } \\
4\end{array}$ & iPad & Mixed \\
\hline 43 & ET12 S & Song (2018) & $\begin{array}{l}\text { Hong Kong } \\
\text { China }\end{array}$ & 53 & $\begin{array}{c}\text { Grade } \\
6\end{array}$ & $\begin{array}{l}\text { iPads, laptop } \\
\text { Smartphone }\end{array}$ & Mixed \\
\hline 44 & ET13 G & Varier et al. (2017) & USA & 42 & $\begin{array}{c}\text { Grades } \\
3-11\end{array}$ & $\begin{array}{l}\text { Digital } \\
\text { devices }\end{array}$ & Qualitative \\
\hline 45 & ET14 M & Wetzel et al. (2020) & Germany & 49 & $\begin{array}{l}\text { Age } \\
12.6\end{array}$ & iPads & Quantitative \\
\hline 46 & JC1 S & Bressler et al. (2019) & USA & 202 & $\begin{array}{l}\text { Grade } \\
\quad 8\end{array}$ & $\begin{array}{c}\text { Tablet } \\
\text { Smartphone }\end{array}$ & Quantitative \\
\hline 47 & JC2 L & Cordero et al. (2018) & Costa Rica & 51 & $\begin{array}{c}\text { Grade } \\
3\end{array}$ & Tablet & Qualitative \\
\hline 48 & JC3 L & Dindar et al. (2015) & Turkey & 303 & $\begin{array}{l}\text { Grade } \\
\quad 7\end{array}$ & Computer & Quantitative \\
\hline 49 & JC4 M & Díaz et al. (2015) & Uruguay & 544 & $\begin{array}{c}\text { Grade } \\
4\end{array}$ & Laptop & Mixed \\
\hline 50 & JC5 M & Gunbas (2015) & Turkey & 128 & $\begin{array}{l}\text { Grade } \\
\quad 6\end{array}$ & Computer & Quantitative \\
\hline 51 & $\mathrm{JC6} \mathrm{S}$ & Hsu et al. (2015) & USA & 54 & $\begin{array}{c}\text { Grade } \\
7\end{array}$ & Computer & Qualitative \\
\hline 52 & $\mathrm{JC7} \mathrm{S}$ & $\begin{array}{l}\text { Jaakkola and } \\
\text { Veermans (2015) }\end{array}$ & Finland & 52 & $\begin{array}{c}\text { Grades } \\
5-6\end{array}$ & Computer & Quantitative \\
\hline 53 & $\mathrm{JC8} \mathrm{S}$ & Johnson et al. (2015) & USA & 250 & $\begin{array}{l}\text { Age } \\
12.5\end{array}$ & Laptop & Quantitative \\
\hline 54 & JC9 L & Liao et al. (2018) & Taiwan & 245 & $\begin{array}{c}\text { Grade } \\
3\end{array}$ & Tablet & Quantitative \\
\hline 55 & JC10 S & Lin et al. (2018) & Taiwan & 55 & $\begin{array}{l}\text { Grade } \\
6\end{array}$ & Tablet & Mixed \\
\hline 56 & JC11 M & Masek et al. (2017) & Australia & 131 & $\begin{array}{c}\text { Year } \\
6\end{array}$ & Tablet & Quantitative \\
\hline 57 & JC12 S & Miller et al. (2016) & USA & 59 & $\begin{array}{l}\text { Grade } \\
\quad 8\end{array}$ & $\begin{array}{l}\text { Laptop } \\
\text { Computer }\end{array}$ & Mixed \\
\hline 58 & $\mathrm{JC} 13 \mathrm{H}$ & Price et al. (2016) & UK & 60 & $\begin{array}{l}\text { Year } \\
5\end{array}$ & $\mathrm{iPad}$ & Qualitative \\
\hline 59 & JC14 L & Shadiev et al. (2018) & Taiwan & 26 & $\begin{array}{l}\text { Age } \\
13-14\end{array}$ & $\begin{array}{l}\text { Tablet } \\
\text { PC }\end{array}$ & Mixed \\
\hline 60 & JC15 LM & $\begin{array}{l}\text { Starkey and Zhong } \\
\text { (2019) }\end{array}$ & New Zealand & 687 & $\begin{array}{l}\text { Years } \\
4-8\end{array}$ & Netbook & Quantitative \\
\hline 61 & JC16 S & $\begin{array}{l}\text { Tho and Yeung } \\
\text { (2018) }\end{array}$ & Hong Kong & 32 & $\begin{array}{c}\text { Grades } \\
7-9\end{array}$ & $\begin{array}{l}\text { Smartphone } \\
\text { Tablet }\end{array}$ & Mixed \\
\hline
\end{tabular}




\begin{tabular}{|c|c|c|c|c|c|c|c|}
\hline 62 & JC17 D & $\begin{array}{l}\text { Witherspoon et al. } \\
\text { (2018) }\end{array}$ & USA & 136 & $\begin{array}{c}\text { Grades } \\
6-8\end{array}$ & Computer & Quantitative \\
\hline 63 & JC18 L & $\begin{array}{l}\text { van Weerdenburg et } \\
\text { al. (2019) }\end{array}$ & Netherlands & 207 & $\begin{array}{c}\text { Grades } \\
4-6\end{array}$ & Computer & Quantitative \\
\hline 64 & JC19 D & $\begin{array}{l}\text { van der Meij and van } \\
\text { der Meij (2016) }\end{array}$ & Germany & 55 & $\begin{array}{l}\text { Grade } \\
7\end{array}$ & Computer & Quantitative \\
\hline
\end{tabular}

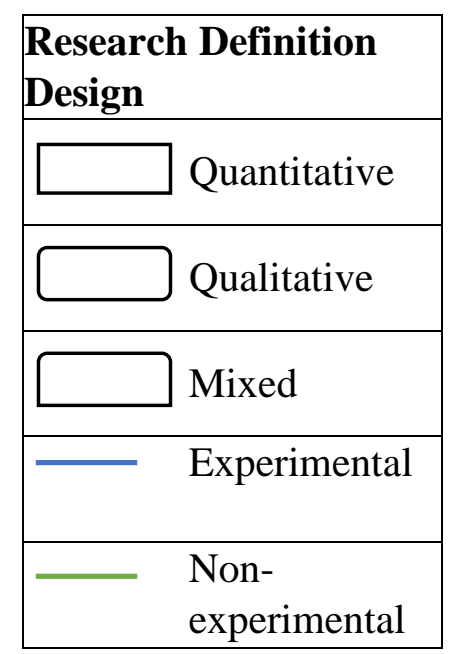

\begin{tabular}{|cl|}
\hline $\begin{array}{c}\text { Journal Definition } \\
\text { Name }\end{array}$ \\
\hline EI & $\begin{array}{l}\text { Education and Information } \\
\text { Technologies }\end{array}$ \\
\hline CE & $\begin{array}{l}\text { Computers \& Education } \\
\text { BJ }\end{array}$ \\
& $\begin{array}{l}\text { British Journal of } \\
\text { Educational Technology }\end{array}$ \\
\hline ET & $\begin{array}{l}\text { Educational Technology } \\
\text { Research and Development }\end{array}$ \\
\hline JC & $\begin{array}{l}\text { Journal of Computer } \\
\text { Assisted Learning }\end{array}$ \\
\hline
\end{tabular}

\begin{tabular}{|cl|}
\hline $\begin{array}{c}\text { Subject } \\
\text { Area }\end{array}$ & Definition \\
\hline L & Language \\
\hline M & Maths \\
\hline S & Science \\
\hline D & Digital Literacy \\
\hline H & Social studies \\
\hline G & Generic \\
\hline T & STEM \\
\hline U & Music \\
\hline E & Media \\
\hline
\end{tabular}




\section{Appendix B : Information Sheet and Consent Forms for Teachers}

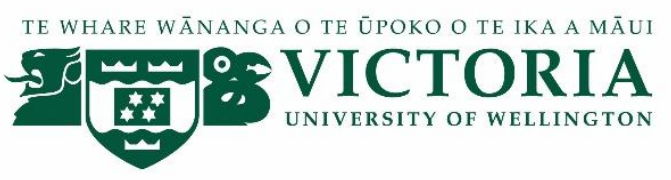

\section{Using Digital Devices in Classroom Learning: A Socio-Material Perspective INFORMATION SHEET FOR TEACHER}

You are invited to take part in this research. Please read this information before deciding whether or not to take part. If you decide to participate, thank you. If you decide not to participate, thank you for considering this request.

\section{Who am I?}

My name is Rana Daoud and I am a Doctoral student in the Faculty of Education at Victoria University of Wellington. This research project is work towards my dissertation.

\section{What is the aim of the project?}

This project is about using digital devices in classroom learning. The purpose of my study is to observe how digital devices are being used in learning activities in primary classrooms in order to understand how digital devices influence classroom learning. My role as a researcher will be to observe specific learning activities designed by you and interviewing the participants in the study.

This research has been approved by the Victoria University of Wellington Human Ethics Committee [0000025742].

\section{How can you help?}

You have been invited to participate because you use digital devices for learning and teaching. If you agree to take part, I will arrange for an initial meeting with you to organise a timeline, choose the learning activities that will be studied. I will also negotiate with you to select (3-6) students to participate in the study and to distribute information sheets and consent forms for them and their families.

I will collect data during term__ as follows:

- I will sit in class and observe learning activities that involve using digital devices.

- During learning activity, I may sometimes ask participating students to talk about what they are thinking when they are using digital devices, why, and what new things have they learnt. The students may choose to use "Screencastify" to record screens and their conversations while doing observed activities. 
- I will collect samples of documents related to the learning activities like teacher plans and task guides beside samples of participating students' works on digital devices that illustrate their learning.

- I will interview you about using digital devices in classroom learning, which will involve one initial interview at the beginning of the term (around 60 minutes), informal conversational interviews during the term (5-10 minutes) and one final interview at the end of the term (around 60 minutes). These interviews will be conducted in school at a time of your convenience.

- I will conduct two group interviews with the participating students. Each will last approximately 30 minutes and will be conducted in the school at a time and location suitable for participants.

I will audio record the interviews with your permission and write it up later. You can choose to not answer any question or stop the interview at any time, without giving a reason. You can withdraw from the study by contacting me at any time before the end of term_. If you withdraw, the information you provided will be destroyed or returned to you.

\section{What will happen to the information you give?}

This research is confidential. This means that the researcher named below will be aware of your identity, but the research data will be combined, and your identity will not be revealed in any reports, presentations, or public documentation.

Only my supervisors and I will read the data, notes or transcript of the interview. The interview transcripts, summaries, data, and any recordings will be kept securely and destroyed on March 2021.

\section{What will the project produce?}

The information from my research will be used in $\mathrm{PhD}$ dissertation and/or academic publications and conferences.

\section{If you accept this invitation, what are the rights of a research participant?}

You do not have to accept this invitation if you don't want to. If you do decide to participate, you have the right to:

- $\quad$ choose not to answer any question;

- $\quad$ ask for the recorder to be turned off at any time during the interview;

- $\quad$ withdraw from the study before the end of term_;

- $\quad$ ask any questions about the study at any time;

- $\quad$ receive a copy of your interview transcript;

- be able to read any reports of this research by emailing the researcher to request a copy. 
If you have any questions or problems, who can you contact?

If you have any questions, either now or in the future, please feel free to contact either:

Student:

Name: Rana Daoud

University email address:

Rana.daoud@vuw.ac.nz
Supervisor:

Name: Dr. Louise Starkey

Role: Senior Lecturer,

School: School of Education

Phone: 044639768

louise.starkey@vuw.ac.nz
Supervisor:

Name: Dr. Hiria McRae

Role: Senior Lecturer,

School: School of Education

Phone: 044639602

hiria.mcrae@vuw.ac.nz

\section{Human Ethics Committee information}

If you have any concerns about the ethical conduct of the research you may contact the Victoria University HEC Convenor: Associate Professor Susan Corbett. Email susan.corbett@vuw.ac.nz or telephone +64-4-463 5480. 


\section{Using Digital Devices in Classroom Learning: A Socio-Material Perspective}

\section{CONSENT FORM FOR TEACHER}

This consent form will be held for three years.

Researcher: Rana Daoud, School of Education, Victoria University of Wellington.

- I have read the Information Sheet and the project has been explained to me. My questions have been answered to my satisfaction. I understand that I can ask further questions at any time.

- I agree to take part in the study.

I understand that:

- I may withdraw from this study at any point before the end of term and any information that I have provided will be returned to me or destroyed.

- $\quad$ The identifiable information I have provided will be destroyed in March 2021.

- Any information I provide will be kept confidential to the researcher and the supervisors.

- I understand that the results will be used for a PhD dissertation and/or academic publications and/or presented to conferences.

- My name will not be used in reports, nor will any information that would identify me.

- I would like a copy of my interview transcript.

Yes $\square \quad$ No

- $\quad$ I would like to receive a copy of the final report and have added my email Yes $\square$ No address below.

Signature of participant:

Name of participant:

Date:

Contact details: 


\section{Appendix C: Information Sheet and Consent Forms for Students}

\section{TE WHARE WĀNANGA O TE ŪPOKO O TE IKA A MĀUI

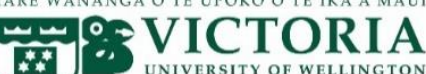

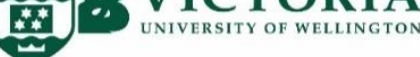 \\ Using Digital Devices in Classroom Learning: A Socio-Material Perspective INFORMATION SHEET FOR STUDENT}

You are invited to take part in this research. Please read this information before deciding whether or not to take part. If you decide to participate, thank you. If you decide not to participate, thank you for considering this request.

\section{Who am I?}

My name is Rana Daoud and I am a Doctoral student in the Faculty of Education at Victoria University of Wellington. This research project is work towards my dissertation.

\section{What is the aim of the project?}

I am doing a research project about digital devices and learning. In your classroom, you use digital devices for different activities. I need your permission to observe you while you are working on the digital devices in the classroom and ask you some questions.

This research has been approved by the Victoria University of Wellington Human Ethics Committee [0000025742].

\section{How can you help?}

You have been invited to participate because you use digital devices for learning in teacher's (xxx) class. I will need to work in your classroom for one term. If you agree to take part, information about your learning and using digital devices will be collected during term__ as follows:

- I will sit in class and observe learning activities that involve using digital devices.

- During learning activity, I may ask you sometimes to talk about what you are thinking when you are using digital devices, why, and what new things have you learnt. You may choose to use "Screencastify" to record screens and your conversations while doing observed activities.

- I will collect samples of documents related to the learning activities like your works on digital devices that illustrate your learning.

- I will conduct two group interviews during term__ with you and other participating students. Each will last approximately 30 minutes and will be conducted in the school at a time and location suitable for participants. 
I will audio record the interviews with your permission and write it up later. You can choose to not answer any question or stop the interview at any time, without giving a reason. You can withdraw from the study by contacting me at any time before the end of term_.. If you withdraw, the information you provided will be destroyed or returned to you.

\section{What will happen to the information you give?}

This research is confidential. This means that the researcher named below will be aware of your identity, but the research data will be combined, and your identity will not be revealed in any reports, presentations, or public documentation.

Only my supervisors and I will read the data, notes or transcript of the interview. The interview transcripts, summaries, data, and any recordings will be kept securely and destroyed on March 2021.

\section{What will the project produce?}

The information from my research will be used in $\mathrm{PhD}$ dissertation and/or academic publications and/or conferences.

\section{If you accept this invitation, what are the rights of a research participant?}

You do not have to accept this invitation if you don't want to. If you do decide to participate, you have the right to:

- $\quad$ choose not to answer any question;

- $\quad$ ask for the recorder to be turned off at any time during the interview;

- withdraw from the study before the end of term_;

- $\quad$ ask any questions about the study at any time;

- $\quad$ receive a summary of your interview;

- be able to read any reports of this research by emailing the researcher to request a copy.

If you have any questions or problems, who can you contact?

If you have any questions, either now or in the future, please feel free to contact either:

\section{Student:}

Name: Rana Daoud

University email address:

Rana.daoud@vuw.ac.nz

\section{Supervisor:}

Name: Dr. Louise Starkey

Role: Senior Lecturer

School: School of Education

Phone: 044639768

louise.starkey@vuw.ac.nz

\section{Supervisor:}

Name: Dr. Hiria McRae

Role: Senior Lecturer

School: School of Education

Phone: 044639602

hiria.mcrae@vuw.ac.nz

\section{Human Ethics Committee information}

If you have any concerns about the ethical conduct of the research you may contact the Victoria University HEC Convenor: Associate Professor Susan Corbett. Email susan.corbett@vuw.ac.nz or telephone +64-4-463 5480. 


\section{Using Digital Devices in Classroom Learning: A Socio-Material Perspective}

\section{CONSENT FORM FOR STUDENT}

This consent form will be held for three years.

Researcher: Rana Daoud, School of Education, Victoria University of Wellington.

- I have read the Information Sheet and the project has been explained to me. My questions have been answered to my satisfaction. I understand that I can ask further questions at any time.

- I agree to take part in the study.

I understand that:

- I may withdraw from this study at any point before the end of term and any information that I have provided will be returned to me or destroyed.

- $\quad$ The identifiable information I have provided will be destroyed in March 2021.

- Any information I provide will be kept confidential to the researcher and the supervisors.

- I understand that the results will be used for a PhD dissertation and/or academic publications and/or presented to conferences.

- My name will not be used in reports, nor will any information that would identify me.

- I would like a summary of my interview.

Yes $\square \quad$ No

- $\quad$ I would like to receive a copy of the final report and have added my email Yes $\square$ No address below.

Signature of participant:

Name of participant:

Date:

Contact details: 


\section{Appendix D: Interview Protocol with Teachers}

The first interview: At the beginning of the term

\section{Estimated Time $60 \mathrm{~min}$.}

The beginning of the interview:

Hello! My name is Rana Daoud; I'm a PhD student at VUW. I'm here to learn about using digital devices in a classroom setting. Thank you for taking the time to talk to me today. The purpose of this interview is to learn how you as a teacher understand learning and the role of digital devices in learning, design learning activities that depend on using digital devices, implement and evaluate learning outcomes that result from these learning activities. There are no right or wrong answers, or desirable or undesirable answers. I would like you to feel comfortable saying what you really think and how you feel. If it's okay with you, I will be tape recording our conversation since it is hard for me to write down everything while simultaneously carrying out an attentive conversation with you. Everything you say will remain confidential, meaning that only myself and my supervisors will be aware of your answers.

Teacher background \& Experience: (warm-up)

- Tell me please about your teaching career?

- Years of experience

- Grades you taught

- Current class

Learning (general):

- How do you understand learning?

- Do you have a special theory/philosophy of learning?

- How do you think students learn best?

Using digital devices (general):

- When did you start using digital devices in learning activities in your classroom?

- What reasons encouraged you to integrate digital devices in your classroom?

- What factors or conditions made it possible for you to integrate digital devices in your classroom?

- What types of learning activities do you use digital devices for?

- So, in general, how do you think using digital devices affect classroom learning?

\section{Learning activity:}

○ Positive influence

- Negative influence

As you know we have discussed before how you intend to use digital devices in your classroom and selected two activities to be followed during this term and you have filled information sheets about these activities. Based on these sheets I have some questions about activity

- Have you used this activity before?
$\circ$ How many times?
- Which grades?

- How do you think this activity will influence student learning?

- Reasons for choosing this activity 
- Factors that affected the decision for choosing this activity (curriculum, students, experience, etc.)

- How did you develop the learning goals of this activity?

- What factors or conditions should exist to achieve the learning goals of this activity?

- You have written that $(x x x)$ device \& $(x x x)$ software will be used in the learning activity so:

$\circ$ Why did you choose these devices?

- Why did you choose this apps/software?

- According to your understanding, what is the role of the digital devices in the activity?

- Why are digital devices important for the learning activity?

- What factors or conditions should exist to use digital devices successfully?

- Can you describe how your students are expected to use digital devices to do this learning activity?

- What obstacles/challenges do you expect you or students will face when using digital devices?

$\circ \quad$ How do you plan to manage these challenges?

- As you clarified in the information sheet, you are expecting the following outcomes as evidence of student learning.

- How are you going to evaluate each of these outcomes?

- In your opinion, can these learning outcomes occur without using digital devices? Why? How?

Thank you very much for your time and for giving me the chance to study learning process in your classroom.

\section{The second interview: At the end of the term}

\section{Estimated Time $60 \mathrm{~min}$.}

PS. there were amendments based on observations

\section{Learning (general):}

- In your opinion, what is learning? How do you define learning?

- So how do you think students learn best?

- What things helped you to develop this opinion about learning?

- Philosophy, theories, readings, relations, experience ...

\section{Learning Activity}

- During the term, your students did the learning activity $(x \mathrm{xx})$ using digital devices, what is your overall evaluation of this learning activity?

- How do you think this activity has influenced student learning?

- What are the learning goals that you had planned for and achieved "learning outcomes"?

- How did you evaluate the learning outcomes?

- What are the factors and conditions that helped to achieve these goals?

- What are the learning goals that were not achieved?

- What are the reasons for not achieving these goals?

- What are the surprising/unexpected outcomes from this activity?

- Why do you think they have occurred? 
- According to your understanding, what was the role of digital devices in the activity?

- Why were digital devices important for the learning activity?

- What were the factors or conditions that help to use digital devices successfully?

- What obstacles/challenges did you or students face when using digital devices?

$\circ$ How did you manage these challenges?

- In your opinion, would these learning outcomes occur without using digital devices? Why? How?

- What changes might you make in the future regarding (learning activity, the way of using digital devices, types of digital devices, software); why?

Thank you very much for your time. It was a great opportunity to study the learning process in your classroom. 


\section{Appendix E: Group Interview Protocol with Students and Think-Aloud Prompts}

\section{Group interview: Sample of questions}

- How do you learn best?

- What is learning in your opinion?

- What do you think about digital devices?

- When do you usually use devices in the classroom? Why?

- What did you learn from activity XXX?

- What new things did you know?

- What new things can you do?

- Did digital devices help/or not help you in this activity?

- Examples

- Were you able to do/know these things without digital devices?

$\circ$ Why?

○ How?

- Examples

- What have other things (or people) helped/hindered your learning in this activity (teacher, book, peer, etc.)?

- What problems have you faced when doing this task/using your digital device?

- How did you overcome these problems?

P.S. Based on the observations, questions about interesting events related to using or learning may be asked.

\section{Think-aloud interview prompts}

Can you talk me through what you have been doing?

How did you know to do this?

What new things have you learnt?

What new things can you do?

What new things have you known?

Who did you learn with?

Who/what has helped you to understand (this learning...)?

How did your digital device help you to learn?

Could you have learnt this without using your digital device?

What problems are you facing while doing this task?

How can you solve these problems? 